\title{
Singularities and Pseudogaps in the Density of States of the Fluctuating Gap Model
}

\author{
Dissertation \\ zur Erlangung des Doktorgrades \\ der Mathematisch-Naturwissenschaftlichen Fakultäten \\ der Georg-August-Universität zu Göttingen
}

vorgelegt von

Lorenz Bartosch

aus Nordhorn

Göttingen 2000 
D7

Referent: Prof. Dr. Peter Kopietz

Korreferent: Prof. Dr. Kurt Schönhammer

Tag der mündlichen Prüfung: 21. Juni 2000 


\section{Contents}

Introduction $\quad$ v

Acknowledgments ix

1 The Fluctuating Gap Model 1

1.1 Fröhlich Hamiltonian and Peierls instability . . . . . . . . . . . . . . 1

1.1.1 Fröhlich Hamiltonian . . . . . . . . . . . . . . . 2

1.2 Euclidean action . . . . . . . . . . . . . . 3

1.3 Ginzburg-Landau theorv . . . . . . . . . . . . . . . . 5

1.3.1 Lindhard function. . . . . . . . . . . . . . . 7

1.3.2 Kohn anomalv in the phonon dispersion . . . . . . . . . 9

1.3.3 Discarding quantum fluctuations . . . . . . . . . . . . 9

1.3.4 Ginzburg-Landau functional . . . . . . . . . . . . . . . 13

1.4 Mean-field theory . . . . . . . . . . . . . . . . . . . . . . . . . . . . . . . 14

1.4.1 Static lattice distortion . . . . . . . . . . . . . . . . . 17

1.4.2 Mean-field Hamiltonian . . . . . . . . . . . . . . . . 18

1.4 .3 The density of states . . . . . . . . . . . . . . . . . . . . . . . . . . . . . . .

1.4.4 Condensation energy . . . . . . . . . . . . . . . . 20

1.4.5 Charge-density wave ................ . . 21

1.5 Breakdown of the mean-field picture . . . . . . . . . . . . . 22

1.5.1 Correlation functions of the order parameter field . . . . . . 22

1.6 The Hamiltonian of the fluctuating gap model . . . . . . . . . . . . . 25

1.6.1 The fluctuating gap model in other physical contexts . . . . 26

2 The Green function and related quantities 27

2.1 The Green function . . . . . . . . . . . . . . . . . . . . . . . . . . . . . . . 27

2.1 .1 Free fermions . . . . . . . . . . . . . . . . . . 28

2.2 Dyson equation and perturbation theory . . . . . . . . . . . . . . . 29

2.2.1 Boundary conditions of the retarded Green function . . . . . . 30

2.3 Second order Born approximation . . . . . . . . . . . 31

2.3 .1 The self-energy . . . . . . . . . . . . . . 32

2.3.2 The density of states and the inverse localization length . . . . 32

2.3 .3 The spectral function . . . . . . . . . . . . 35 
2.4 Non-Abelian Schwinger-ansatz . . . . . . . . . . . . . . . . . . . . . . . . . 35

2.5 Riccati equation . . . . . . . . . . . . . . . . . . 39

2.5.1 The $S$-matrix for constant potentials . . . . . . . . . . . . . 40

2.5.2 Initial conditions . . . . . . . . . . . . . . . . . . 40

2.5.3 The case of a discrete spectrum . . . . . . . . . . . . . 41

2.6 Integrated averaged Green function $\Gamma(\omega)$. . . . . . . . . . . . . 43

2.7 Gauge invariance . . . . . . . . . . . . . . . . . 46

2.8 Lvapunov exponent and localization length . . . . . . . . . . . . . . 48

2.8.1 Thouless formula . . . . . . . . . . . . . . . . 48

2.8.2 Localization length at $\omega=0$ for real $\Delta(x) \ldots \ldots$. . . . . 50

2.9 Eilenberger and pseudo-Schrödinger equation . . . . . . . . . . 50

3 Exact results

3.1 The white noise limit . . . . . . . . . . . . . 55

3.1.1 Equality of the integrated density of states and the stationary probability flux ................. . 56 56

3.1.2 White noise and the Fokker-Planck equation . . . . . . 57

3.1.3 The density of states in the white noise limit . . . . . . . . 60

3.1.4 The commensurate case without forward scattering . . . . . 60

3.1.5 Solving the general case with arbitrary parameters $D_{R}, D_{I}, D_{V}$

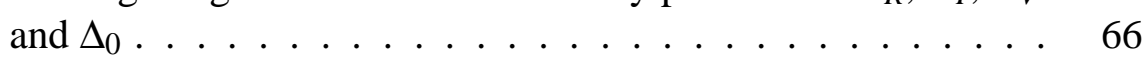

3.1 .6 The incommensurate case . . . . . . . . . . . 68

3.2 Infinite correlation lengths . . . . . . . . . . . . . . . 71

3.2.1 The commensurate case . . . . . . . . . . . 71

3.2.2 Incommensurate case . . . . . . . . . . . . . . 72

4 Finite correlation lengths

4.1 Singularities in the density of states . . . . . . . . . . . . . 75

4.2 Numerical algorithm . . . . . . . . . . . . . . . . 76

4.2.1 Generation of disorden . . . . . . . . . . . . . 79

4.2 .2 Results . . . . . . . . . . . . . . . . 80

4.3 Phase fluctuations onlv . . . . . . . . . . . . . . . . . . . . 84

4.3.1 Density of states and inverse localization length . . . . . . . 87

4.3.2 Pauli paramagnetic susceptibility . . . . . . . . . . . . . . . . 91

4.3 .3 Thermodynamic quantities . . . . . . . . . . . . . 93

$\begin{array}{lr}\text { Conclusion } & 97\end{array}$

\begin{tabular}{|ll}
\hline Gradient expansion of the free energy & 99
\end{tabular}

\begin{tabular}{ll}
\hline G Generation of Colored Noise & 105
\end{tabular} 


\section{Introduction}

As the temperature is lowered, some inorganic and organic conductors with a highly anisotropic crystal and electronic structure become unstable and undergo a Peierls transition, i.e. they develop a charge-density wave. This instability is due to their quasi one-dimensional nature which results in a (perfectly) nested Fermi surface. A qualitative understanding of the Peierls instability can already be gained by coupling independent electrons to phonons and treating the phonon field which can be identified as the order parameter field in a mean-field picture [31, 36, 37, 55, 72, 75, 76]. However, because of reduced dimensionality, fluctuations of the order parameter field $\Delta(x)$ are crucial and significant deviations are to be expected.

In a seminal paper, Lee, Rice and Anderson [57] introduced the one-dimensional so-called fluctuating gap model (FGM), in which fluctuations of the phonon field are described by a static disorder potential. Calculating the leading-order correction of the electronic self energy of an incommensurate chain which is described by a complex order parameter field with $\langle\Delta(x)\rangle=0$ and $\left\langle\Delta(x) \Delta^{*}\left(x^{\prime}\right)\right\rangle=\Delta_{s}^{2} e^{-\left|x-x^{\prime}\right| / \xi}$, where $\xi$ is the temperature-dependent correlation length, Lee, Rice, and Anderson obtained an approximate expression for the density of states (DOS), showing a suppression of the DOS near the Fermi energy, which is called a pseudogap.

A few years later, Sadovskii [78] apparently obtained an exact expression for the Green function of the FGM using Gaussian statistics for the higher correlation functions of the order parameter field which he could assume to be real or complex, referring to a band filling being commensurate or incommensurate with the underlying lattice. Recently, the experimental observation of a pseudo-gap state in the overdoped cuprates above the superconducting phase transition lead to a reincarnation of the FGM and Sadovskii's exact solution [80, 81] in the field of high-temperature superconductivity. However, the revived interest in Sadovskii's solution also brought to light a subtle error in this solution [87] which questions not only the solution itself, but also the work based on it.

Besides the limit $\xi \rightarrow \infty$ where Sadovskii's solution is indeed exact [54, 87], Sadovskii's solution can also be easily tested in the white-noise limit $\xi \rightarrow 0$, keeping $D \equiv \Delta_{s}^{2} \xi$ constant, such that $\left\langle\Delta(x) \Delta^{*}\left(x^{\prime}\right)\right\rangle=2 D \delta\left(x-x^{\prime}\right)$. Solving a stationary Fokker-Planck equation, Ovchinnikov and Erikhman [71] obtained an exact expression for the DOS for real $\Delta(x)$. They showed that for small $\omega$ and $\langle\Delta(x)\rangle=0$, the 
DOS diverges as $\langle\rho(\omega)\rangle \propto\left|\omega \ln ^{3}\right| \omega||^{-1}$. Singularities of this type at the band center of a random Hamiltonian have been discovered by Dyson [19] in the fifties and have recently also been found in one-dimensional spin-gap systems [27, 62]. It is important to note that in the FGM the singularity is a consequence of phase resonance, and is not related to concrete probability properties of $\Delta(x)[58,68]$. In particular, the singularity is not an artifact of the exactly solvable limit $\xi \rightarrow 0$ considered in Ref. [71]. As argued in Ref. [8], it is therefore reasonable to expect that for any $\xi<\infty$ the average DOS of the FGM exhibits a singularity at $\omega=0$. This general argument is in disagreement with Sadovskii's solution [78] which for large but finite $\xi$ shows a pseudogap and no singularity. In this work we shall reexamine the DOS of the FGM which determines the whole thermodynamics of the FGM and resolve the above contradictions.

We begin this work in Chapter 1 with an elementary introduction to the Peierls effect of both commensurate and incommensurate Peierls chains. Starting from a Fröhlich Hamiltonian which describes a one-dimensional electron-phonon system, we obtain a Euclidean action which after integrating out the fermionic degrees of freedom can be approximated by a static free energy functional of the phonon field. Expanding this functional for small fields and keeping only the relevant terms, we are left with the well-known Ginzburg-Landau functional of a one-dimensional Peierls system. We will see that correction terms to this Ginzburg-Landau functional are already important within a mean-field picture away from criticality. In the mean-field approximation, we can explain the experimentally observed static lattice distortion and the accompanied charge-density wave. Finally, we will discuss the importance of fluctuations of the order parameter field and introduce the fluctuating gap model (FGM).

Chapter 2 focuses on the one-particle Green function of the FGM. After calculating the Green function in the leading-order Born approximation which reproduces the result obtained by Lee, Rice and Anderson, we will develop a formally exact nonperturbative expression of the Green function as a functional of the disorder potentials based on a non-Abelian generalization of the Schwinger-ansatz. To calculate the DOS and inverse localization length, the introduction of phase variables will turn out to be very convenient. While one phase variable is simply related to the integrated DOS and satisfies a non-linear equation of motion which is equivalent to a Riccati equation, the other phase variable is related to the inverse localization length and can be expressed in terms of the first phase variable. These equations of motions will serve as the starting point for detailed calculations of the DOS and inverse localization length for various probability distributions of the disorder potentials in the next chapters. It will turn out that physical quantities like the DOS or inverse localization length are left invariant under a gauge transformation which maps phase fluctuations of the order parameter field onto an effective forward scattering potential and vice versa. We will make use of this gauge-transformation in the following chapters.

In Chapter 3 we will review known exact results of the DOS and inverse localization length in the limit of infinite correlation lengths and in the white noise limit. Generalizing the phase formalism developed in Ref. [58] such that $\Delta(x)$ is allowed to be complex, we will derive a linear fourth-order Fokker-Planck equation previously 
only obtained within the framework of the method of supersymmetry [42]. The solution of this stationary Fokker-Planck equation encapsulates all known results for the DOS and inverse localization length of the FGM in the white noise limit including the above mentioned Dyson singularity in the Ovchinnikov and Erikhman limit. Results for the case of infinite correlation lengths will finally be obtained by averaging the DOS and the inverse localization length calculated for a constant disorder potential over an appropriate probability distribution of the disorder potentials.

The case of finite correlation lengths of the order parameter field will be attacked in Chapter 4 Considering the equation of motion related to the integrated DOS, we will first argue that we expect for any finite $\xi$ a Dyson singularity in the DOS. We will then set up an algorithm based on the equations of motion derived in Chapter 2 which will allow for a simultaneous numerical calculation of the DOS and inverse localization length for arbitrary disorder potentials with unprecedented accuracy. For complex $\Delta(x)$, Sadovskii's solution is not too far off from our numerical solution. In particular, for large correlation lengths $\Delta_{s} \xi \gg 1$, the DOS at the Fermi energy vanishes as $\rho(0) \propto\left(\Delta_{s} \xi\right)^{-0.64}$ instead of $\rho(0) \propto\left(\Delta_{s} \xi\right)^{-1 / 2}$, as predicted by Sadovskii. However, for real $\Delta$, we will find a pseudogap in the DOS for $\Delta_{s} \xi \gg 1$ which for any finite $\xi$ is overshadowed by a Dyson singularity of the form $\rho(\omega)=A\left|\omega \ln ^{\alpha+1}\right| \omega||^{-1}$, where $A$ and the exponent $\alpha$ depend on the correlation length $\xi$. As the correlation length $\xi$ increases, $\alpha$ assumes the finite value $\alpha=0.41$, but the weight of the Dyson singularity vanishes with increasing correlation length. At the end of Chapter 4 we shall also discuss the case of only phase fluctuations of the order parameter which applies to sufficiently low temperatures where the amplitude of the order parameter is confined to a narrow region around $\Delta_{s}$ such that Gaussian statistics do not apply any more. We will find exact analytic results for the DOS and inverse localization length and we will also calculate the low-temperature Pauli paramagnetic susceptibility and the electronic low-temperature specific heat.

In Appendix A we will use the formalism developed in Chapter 2 to obtain a gradient expansion in the order parameter field of the local DOS and the related free energy functional. Such a gradient expansion is well-known in the semi-classical theory of superconductivity. Finally, in Appendix B we will describe the algorithm used in Chapter 4 to generate Gaussian colored noise in detail. We will also briefly discuss an alternative method which can be used to generate disorder with an arbitrary given spectrum. 



\section{Acknowledgments}

During the past years, I have benefited greatly from numerous discussions with my advisor Peter Kopietz. He shared with me his fascination for physics, introduced me to the art of writing papers, and always encouraged and supported me in following up my own ideas. Working with Peter has turned out to be very fruitful and we have also enjoyed ourselves outside the office. I would like to thank him for all that.

I would like to express my considerable gratitude to my teacher Prof. Dr. Kurt Schönhammer who initiated my interest in quantum mechanics with his lectures. KS always showed a great interest in my work and his ideas also had an influence on it.

I am deeply indebted to Sudip Chakravarty for his hospitality during the fall and winter quarter of 1997/98 at the Department of Physics of the University of California, Los Angeles. His intuitive way of doing physics impressed me much.

For some problems, I had to consult experts from out of Göttingen. I would particularly like to thank Roland Hayn for answering some questions on his papers and for providing me with some unpublished notes.

My special thanks go to Nias Djonlagić who was always very helpful and, in many discussions, contributed to solving small but important problems. With computerproblems, I could always count on Jürgen Holm and Thomas Östreich whose advice was often worth more than any reference book I could find. I would like to thank them for their patience.

In one way or the other, I have also benefited from discussions with Volker Meden, Ivan Spremo, Rudolf Hausmann, Axel Völker, Rauno Recklebe, and Tom Busche. To these people, and to many other people who shared my everyday life, I am extremely grateful.

This work was financially supported by the DFG (Grant No. Ko 1442/4-1). 



\section{Chapter 1}

\section{The Fluctuating Gap Model}

This chapter serves as an introduction into Peierls systems which due to their quasi one-dimensional nature and the presence of electron-phonon interactions develop a static lattice distortion and a charge-density wave. After a microscopic derivation of a (generalized) Ginzburg-Landau functional, we briefly discuss the Peierls instability in a mean-field picture. It follows a discussion of fluctuations of the order parameter which due to reduced dimensionality are very important for an adequate description of Peierls chains. Finally, we introduce the fluctuating gap model (FGM) as a lowenergy model which takes into account these fluctuations. The FGM has a wide range of applications.

\subsection{Fröhlich Hamiltonian and Peierls instability}

The formation of periodic lattice distortions and charge-density waves in Peierls chains is due to the electron-phonon interaction in these quasi one-dimensional materials [31, 36, 37, 55, 72]. Since particle-hole excitations with momentum $2 k_{F}$ are possible for very small excitation energies, the Lindhard density-density response function exhibits a singularity at $q=2 k_{F}$. Kohn showed that this singularity should be conveyed into a kink in the phonon spectrum [47]. While these Kohn anomalies are rather weak in isotropic materials, they can lead to a substantial alteration to the phonon dispersion in quasi one-dimensional materials with a topology of the Fermi surface which shows perfect nesting. At low enough temperatures, the renormalized phonon mode at $2 k_{F}$ can scale all the way down to zero, i.e. become gapless. This process is called softening of the phonon mode. Since $\omega_{\text {ren }}\left(2 k_{F}\right) \rightarrow 0$, a static lattice distortion with wave vector $2 k_{F}$ may now arise. Simultaneously, there is a formation of a charge density wave. As a consequence, the discrete translational invariance is broken. The same physics can also be described by considering the thermodynamics of a Peierls system. This approach will also allow to go beyond a mean-field picture and will therefore be followed here. 


\subsubsection{Fröhlich Hamiltonian}

A Hamiltonian to describe a one-dimensional electron-phonon system was proposed in 1954 by Fröhlich [31]:

$$
\mathcal{H}=\sum_{k, \sigma} \varepsilon_{k} c_{k, \sigma}^{\dagger} c_{k, \sigma}+\sum_{q} \omega_{q} b_{q}^{\dagger} b_{q}+\sum_{q} \frac{g_{q}}{\sqrt{L}} \hat{\rho}_{q}^{\dagger}\left(b_{q+K(q)}+b_{-q-K(q)}^{\dagger}\right) .
$$

The system has length $L=N a$ where $a$ is the lattice spacing, and periodic boundary conditions are assumed. $c_{k}^{\dagger}$ and $c_{k}$ are creation and annihilation operators of fermions with momentum $k$, spin $\sigma$, and energy $\varepsilon_{k}$. Their anticommutators are given by

$$
\left\{c_{k, \sigma}, c_{k^{\prime}, \sigma^{\prime}}\right\}=0, \quad\left\{c_{k, \sigma}, c_{k^{\prime}, \sigma^{\prime}}^{\dagger}\right\}=\delta_{\sigma, \sigma^{\prime}} \delta_{k, k^{\prime}} \mathbf{1}
$$

While $\varepsilon_{k}=k^{2} / 2 m$ for free electrons, in the tight-binding approximation one has $\varepsilon_{k}=$ $-2 t \cos k a$.

The second term in the Fröhlich Hamiltonian (1.1) describes phonons with phonon dispersion $\omega_{q} . q$ is confined to the first Brillouin zone and for a chain with only nearestneighbor interactions we have (see for example Ref. [6]) $\omega_{q}=2 \omega_{0} \sin |q a / 2|$. The creation and annihilation operators $b_{q}^{\dagger}$ and $b_{q}$ of the phonons satisfy the commutation relations

$$
\left[b_{q}, b_{q^{\prime}}\right]=0, \quad\left[b_{q}, b_{q^{\prime}}^{\dagger}\right]=\delta_{q, q^{\prime}} \mathbf{1} .
$$

Finally, the last term in Eq. (1.1) models the interaction of the phonon system with the fermions. The phonons are linearly coupled via the electron-phonon coupling constant $g_{q}$ to the Fourier components of the electron density

$$
\hat{\rho}_{q}^{\dagger} \equiv \sum_{k, \sigma} c_{k+q, \sigma}^{\dagger} c_{k, \sigma}
$$

Treating the electrons in the free electron approximation, $q$ is not restricted to the first Brillouin zone $(-\pi / a, \pi / a)$. To assure that the phonon momentum lies in the first Brillouin zone, we define a reciprocal lattice vector $K(q)$ such that $(-\pi / a<q-$ $K(q)<\pi / a)$. In the following we will identify $q$ with $q-K(q)$ for phonons such that we may formally omit $K(q)$.

\section{Relation of phonon operators to operators of lattice displacements}

The phonon operators $b_{q}$ and $b_{q}^{\dagger}$ are directly related to the operators of the normal coordinates $u_{q}$ of the lattice system by

$$
u_{q}=\left(\frac{1}{2 M \omega_{q}}\right)^{1 / 2}\left(b_{q}+b_{-q}^{\dagger}\right) \text {. }
$$

Here, $M$ is the ionic mass. The lattice displacement operators of the ions at $x_{n}=n a$ are given by its Fourier transform,

$$
u\left(x_{n}\right)=\sum_{q} e^{i q x_{n}}\left(\frac{1}{2 N M \omega_{q}}\right)^{1 / 2}\left(b_{q}+b_{-q}^{\dagger}\right) .
$$


As we will see later, in a mean-field picture the Peierls transition will lead to a nonvanishing expectation value $\left\langle u\left(x_{n}\right)\right\rangle$ which implies that the system exhibits a static lattice distortion.

\subsection{Euclidean action}

In an Euclidean functional integral approach, the Fröhlich Hamiltonian is conveyed into the action (see, for example, Negele and Orland [70])

$$
S\left\{\psi^{*}, \psi ; b^{*}, b\right\}=S_{\mathrm{el}}\left\{\psi^{*}, \psi\right\}+S_{\mathrm{ph}}\left\{b^{*}, b\right\}+S_{\mathrm{int}}\left\{\psi^{*}, \psi ; b^{*}, b\right\},
$$

where

$$
\begin{aligned}
& S_{\mathrm{el}}\left\{\psi^{*}, \psi\right\}=\beta \sum_{k, \tilde{\omega}_{n}, \sigma} \psi_{k, \tilde{\omega}_{n}, \sigma}^{*}\left[i \tilde{\omega}_{n}-\tilde{\varepsilon}_{k}\right] \psi_{k, \tilde{\omega}_{n}, \sigma}, \\
& S_{\mathrm{ph}}\left\{b^{*}, b\right\}=-\beta \sum_{q, \omega_{m}} b_{q, \omega_{m}}^{*}\left[i \omega_{m}-\omega_{q}\right] b_{q, \omega_{m}}, \\
& S_{\mathrm{int}}\left\{\psi^{*}, \psi ; b^{*}, b\right\}=\beta \sum_{q, \omega_{m}} \frac{g_{q}}{\sqrt{L}}\left(\sum_{k, \tilde{\omega}_{n}, \sigma} \psi_{k+q, \tilde{\omega}_{n}+\omega_{m}, \sigma}^{*} \psi_{k, \tilde{\omega}_{n}, \sigma}\right) \\
& \times\left[b_{q, \omega_{m}}+b_{-q,-\omega_{m}}^{*}\right] .
\end{aligned}
$$

Here, $\beta \equiv 1 / k_{B} T$ is the inverse temperature and $\tilde{\varepsilon}_{k} \equiv \varepsilon_{k}-\mu$ is the energy dispersion reduced by the chemical potential $\mu$. While the conjugated Grassmann variables $\psi_{k, \tilde{\omega}_{n}}$ and $\psi_{k, \tilde{\omega}_{n}}^{*}$ describe fermions with momentum $k$ and fermionic Matsubara frequency $\tilde{\omega}_{n} \equiv(2 n+1) \pi / \beta, b_{q, \omega_{m}}$ and $b_{q, \omega_{m}}^{*}$ are complex (bosonic) phonon fields with momentum $q$ and bosonic Matsubara frequency $\omega_{m} \equiv 2 \pi m / \beta$. Both $n$ and $m$ are integers. In terms of the above action, the partition function reads

$$
Z=\int \mathcal{D}\left\{\psi^{*}, \psi\right\} \mathcal{D}\left\{b^{*}, b\right\} \exp \left[-S\left\{\psi^{*}, \psi ; b^{*}, b\right\}\right],
$$

where $\mathcal{D}\left\{\psi^{*}, \psi\right\}$ and $\mathcal{D}\left\{b^{*}, b\right\}$ are appropriately normalized fermionic and bosonic integration measures [70]. Thermal averages of a Euclidean time-ordered operator $\mathcal{T}\left[F\left\{c^{\dagger}, c ; b^{\dagger}, b\right\}\right]$ are given by

$$
\begin{aligned}
\left\langle\mathcal{T}\left[F\left\{c^{\dagger}, c ; b^{\dagger}, b\right\}\right]\right\rangle=\frac{1}{z} \int & \mathcal{D}\left\{\psi^{*}, \psi\right\} \mathcal{D}\left\{b^{*}, b\right\} \\
& F\left\{\psi^{*}, \psi ; b^{*}, b\right\} \exp \left[-S\left\{\psi^{*}, \psi ; b^{*}, b\right\}\right] .
\end{aligned}
$$

The following variable transformation turns out to be convenient:

$$
\begin{aligned}
\phi_{q, \omega_{m}} & \equiv \frac{g_{q}}{\sqrt{L}}\left(b_{q, \omega_{m}}+b_{-q,-\omega_{m}}^{*}\right), \\
\eta_{q, \omega_{m}} & \equiv-i \frac{g_{q}}{\sqrt{L}}\left(b_{q, \omega_{m}}-b_{-q,-\omega_{m}}^{*}\right) .
\end{aligned}
$$


Note that by definition $\phi_{q, \omega_{m}}^{*}=\phi_{-q,-\omega_{m}}$ and $\eta_{q, \omega_{m}}^{*}=\eta_{-q,-\omega_{m}}$. Since the action considered as a functional of $\psi^{*}, \psi, \phi$ and $\eta$ is Gaussian in $\eta$, the respecting integral may easily be done 1 resulting in

$$
S\left\{\psi^{*}, \psi ; \phi\right\}=S_{\mathrm{el}}\left\{\psi^{*}, \psi\right\}+S_{\mathrm{ph}}\{\phi\}+S_{\mathrm{int}}\left\{\psi^{*}, \psi ; \phi\right\},
$$

where $S_{\mathrm{el}}\left\{\psi^{*}, \psi\right\}$ is unchanged and

$$
\begin{aligned}
S_{\mathrm{ph}}\{\phi\} & =\frac{1}{2} \beta L \sum_{q, \omega_{m}} \frac{1}{\left|g_{q}\right|^{2}} \phi_{q, \omega_{m}}^{*}\left[\frac{\omega_{m}^{2}+\omega_{q}^{2}}{\omega_{q}}\right] \phi_{q, \omega_{m}}, \\
S_{\mathrm{int}}\left\{\psi^{*}, \psi ; \phi\right\} & =\beta \sum_{q, \omega_{m}}\left(\sum_{k, \tilde{\omega}_{n}} \psi_{k+q, \tilde{\omega}_{n}+\omega_{m}} \psi_{k, \tilde{\omega}_{n}}\right) \phi_{q, \omega_{m}} .
\end{aligned}
$$

So far, no approximation has been made. In the following, we will restrict ourselves to the low-energy physics of the weak-coupling limit, so that only fermions in the vicinity of the Fermi energy are involved. In this case the Fermi energy may be linearized around the two Fermi points, such that it assumes the form

$$
\tilde{\varepsilon}_{k}=v_{F}\left(|k|-k_{F}\right) \text {. }
$$

To separate right- and left-moving Fermions, let us introduce the spinor field

$$
\bar{\psi}_{k, \tilde{\omega}_{n}, \sigma} \equiv\left(\begin{array}{c}
\psi_{+, k, \tilde{\omega}_{n}, \sigma} \\
\psi_{-, k, \tilde{\omega}_{n}, \sigma}
\end{array}\right) \equiv\left(\begin{array}{c}
\psi_{k_{F}+k, \tilde{\omega}_{n}, \sigma} \\
\psi_{-k_{F}+k, \tilde{\omega}_{n}, \sigma}
\end{array}\right)
$$

and its conjugated counterpart

$$
\bar{\psi}_{k, \tilde{\omega}_{n}, \sigma}^{\dagger} \equiv\left(\psi_{+, k, \tilde{\omega}_{n}, \sigma}^{*}, \psi_{-, k, \tilde{\omega}_{n}, \sigma}^{*}\right) \equiv\left(\psi_{k_{F}+k, \tilde{\omega}_{n}, \sigma}^{*}, \psi_{-k_{F}+k, \tilde{\omega}_{n}, \sigma}^{*}\right) .
$$

The electronic part of the action may be easily rewritten in terms of these spinor fields and the inverse non-interacting Matsubara Green function

$$
\mathbf{G}_{0}^{-1}\left(k, \tilde{\omega}_{n}\right) \equiv\left(\begin{array}{cc}
i \tilde{\omega}_{n}-v_{F} k & 0 \\
0 & i \tilde{\omega}_{n}+v_{F} k
\end{array}\right)
$$

Since the momentum transfer of the phonons is either small compared with the Fermi momentum or approximately $2 k_{F}$, we decompose $\phi_{q, \omega_{m}}$ according to

$$
\mathbf{V}_{q, \omega_{m}} \equiv\left(\begin{array}{cc}
V_{q, \omega_{m}} & \Delta_{q, \omega_{m}} \\
\Delta_{-q,-\omega_{m}}^{*} & V_{q, \omega_{m}}
\end{array}\right) \equiv\left(\begin{array}{cc}
\phi_{q, \omega_{m}} & \phi_{q+2 k_{F}, \omega_{m}} \\
\phi_{q-2 k_{F}, \omega_{m}} & \phi_{q, \omega_{m}}
\end{array}\right),
$$

such that $|q|<k_{F}$. While $\phi_{q, \omega_{m}}^{*}=\phi_{-q,-\omega_{m}}$ directly translates into $V_{q, \omega_{m}}^{*}=V_{-q,-\omega_{m}}$, a similar relation for $\Delta_{q, \omega_{m}}$ does only hold if $4 k_{F}$ is a reciprocal lattice vector.

\footnotetext{
${ }^{1}$ Physically this is due to the fact that we consider only non-interacting phonons which are coupled to the space-coordinates of the underlying lattice.
} 
$\Delta_{q, \omega_{m}}^{*}=\Delta_{-q,-\omega_{m}}$ is therefore only true for a half-filled band for which $\pi / a=2 k_{F}$. We will refer to this case as the commensurate case. The more general case for which $k_{F} a / \pi$ is an other fractional number is also called commensurate but will not be discussed here. In the incommensurate case, for which $k_{F} a / \pi$ is well separated from any simple fractional number, all $\Delta_{q, \omega_{m}}^{*}$ and $\Delta_{-q^{\prime},-\omega_{m^{\prime}}}$ are independent. We will see in this work that commensurate and incommensurate Peierls systems can have very different physical properties.

Defining the matrices $\mathbf{G}_{0}^{-1}$ and $\mathbf{V}$ via

$$
\begin{aligned}
\left(\mathbf{G}_{0}^{-1}\right)_{k, k^{\prime}, \tilde{\omega}_{n}, \tilde{\omega}_{n^{\prime}}} & \equiv \delta_{k, k^{\prime}} \delta_{\tilde{\omega}_{n}, \tilde{\omega}_{n^{\prime}}} \mathbf{G}_{0}^{-1}\left(k, \tilde{\omega}_{n}\right) \\
(\mathbf{V})_{k, k^{\prime}, \tilde{\omega}_{n}, \tilde{\omega}_{n^{\prime}}} & \equiv \mathbf{V}_{k-k^{\prime}, \tilde{\omega}_{n}-\tilde{\omega}_{n^{\prime}}}
\end{aligned}
$$

our action turns into

$$
S\left\{\bar{\psi}^{\dagger}, \bar{\psi} ; V, \Delta, \Delta^{*}\right\}=S_{\mathrm{el}-\mathrm{ph}}\left\{\bar{\psi}^{\dagger}, \bar{\psi} ; V, \Delta, \Delta^{*}\right\}+S_{\mathrm{ph}}\left\{V, \Delta, \Delta^{*}\right\}
$$

where

$$
\begin{aligned}
S_{\mathrm{el}-\mathrm{ph}}\left\{\bar{\Psi}^{\dagger}, \bar{\Psi} ; V, \Delta, \Delta^{*}\right\} & =\beta \sum_{k, k^{\prime}, \tilde{\omega}_{n}, \tilde{\omega}_{n^{\prime}}, \sigma} \bar{\Psi}_{k, \tilde{\omega}_{n}, \sigma}^{\dagger}\left(\mathbf{G}_{0}^{-1}-\mathbf{V}\right)_{k, k^{\prime}, \tilde{\omega}_{n}, \tilde{\omega}_{n^{\prime}}} \bar{\Psi}_{k^{\prime}, \tilde{\omega}_{n^{\prime}}, \sigma} \\
S_{\mathrm{ph}}\left\{V, \Delta, \Delta^{*}\right\} & =\frac{1}{2} \beta L \sum_{q, \omega_{m}} \frac{1}{\left|g_{q}\right|^{2}}\left[\frac{\omega_{m}^{2}+\omega_{q}^{2}}{\omega_{q}}\right] V_{q, \omega_{m}}^{*} V_{q, \omega_{m}} \\
& +\frac{1}{c} \beta L \sum_{q, \omega_{m}} \frac{1}{\left|g_{2 k_{F}+q}\right|^{2}}\left[\frac{\omega_{m}^{2}+\omega_{2 k_{F}+q}^{2}}{\omega_{2 k_{F}+q}}\right] \Delta_{q, \omega_{m}}^{*} \Delta_{q, \omega_{m}}
\end{aligned}
$$

and

$$
c \equiv \begin{cases}2, & \text { commensurate case (half-filled band) } \\ 1, & \text { incommensurate case }\end{cases}
$$

While in the incommensurate case $\pm 2 k_{F}$ lie (up to a reciprocal lattice vector) inside the first Brillouin zone, $\pm 2 k_{F}$ lie directly on the border of the first Brillouin zone in the commensurate case. In this case the factor of $1 / 2$ in the last line in Eq. (1.27) avoids overcounting.

\subsection{Ginzburg-Landau theory}

Since the action describing the Peierls chain is only Gaussian in both the Fermion and phonon fields, either of them can easily be integrated out. To determine the phonon statistics and the related lattice deformations, let us first integrate out the fermionic fields. $S_{\mathrm{el}-\mathrm{ph}}\left\{\bar{\psi}^{\dagger}, \bar{\psi} ; V, \Delta, \Delta^{*}\right\}$ then turns into

$$
S_{\text {ph,int }}\left\{V, \Delta, \Delta^{*}\right\}=-\left[\ln \int \mathcal{D}\left\{\psi^{*}, \psi\right\} \exp \left(-S_{\text {el-ph }}\left\{\bar{\psi}^{\dagger}, \bar{\psi} ; V, \Delta, \Delta^{*}\right\}\right)\right.
$$




$$
\begin{aligned}
& \left.-\ln \int \mathcal{D}\left\{\psi^{*}, \psi\right\} \exp \left(-S_{\mathrm{el}-\mathrm{ph}}\left\{\bar{\psi}^{\dagger}, \bar{\psi} ; 0,0,0\right\}\right)\right] \\
=- & s \ln \operatorname{det}\left[\mathbf{1}-\mathbf{G}_{0} \mathbf{V}\right]=-s \operatorname{Tr} \ln \left[\mathbf{1}-\mathbf{G}_{0} \mathbf{V}\right],
\end{aligned}
$$

where the logarithm of the matrix $\left[\mathbf{1}-\mathbf{G}_{0} \mathbf{V}\right]$ is defined by its power series and $s$ counts the number of spin states and is defined as

$$
s \equiv \begin{cases}2, & \text { fermions with spin } 1 / 2 \\ 1, & \text { spinless fermions }\end{cases}
$$

Expanding the logarithm up to terms of fourth order, we get

$$
S_{\text {ph,int }}\left\{V, \Delta, \Delta^{*}\right\}=\frac{s}{2} \operatorname{Tr}\left(\mathbf{G}_{0} \mathbf{V}\right)^{2}+\frac{s}{4} \operatorname{Tr}\left(\mathbf{G}_{0} \mathbf{V}\right)^{4} .
$$

Neglecting for simplicity cross-terms between $V$ and $\Delta, S_{\text {ph,int }}\left\{V, \Delta, \Delta^{*}\right\}$ assumes the form 2

$$
S_{\text {ph,int }}\left\{V, \Delta, \Delta^{*}\right\}=S_{\text {ph,int }}\{V\}+S_{\text {ph,int }}\left\{\Delta, \Delta^{*}\right\}
$$

with

$$
\begin{aligned}
S_{\mathrm{ph}, \text { int }}\{V\} & =-\frac{s}{2} \beta L \sum_{q, \omega_{m}} \Pi_{0}\left(q, \omega_{m}\right) V_{q, \omega_{m}}^{*} V_{q, \omega_{m}}, \\
S_{\mathrm{ph}, \text { int }}\left\{\Delta, \Delta^{*}\right\} & =-s \beta L \sum_{q, \omega_{m}} \Pi_{0}^{2 k_{F}}\left(q, \omega_{m}\right) \Delta_{q, \omega_{m}}^{*} \Delta_{q, \omega_{m}} \\
& +\frac{s}{2} \beta L \sum_{q_{i}, \omega_{m_{i}}} U_{4}\left(q_{i}, \omega_{m_{i}}\right) \Delta_{-q_{4},-\omega_{m_{4}}}^{*} \Delta_{-q_{3},-\omega_{m_{3}}}^{*} \Delta_{q_{2}, \omega_{m_{2}}} \Delta_{q_{1}, \omega_{m_{1}}}
\end{aligned}
$$

and

$$
\begin{aligned}
\Pi_{0}\left(q, \omega_{m}\right) & \equiv-\frac{1}{\beta L} \sum_{\alpha} \sum_{k, \tilde{\omega}_{n}} G_{0}^{\alpha}\left(k, \tilde{\omega}_{n}\right) G_{0}^{\alpha}\left(k+q, \tilde{\omega}_{n}+\omega_{m}\right), \\
\Pi_{0}^{2 k_{F}}\left(q, \omega_{m}\right) & \equiv-\frac{1}{\beta L} \sum_{k, \tilde{\omega}_{n}} G_{0}^{-}\left(k, \tilde{\omega}_{n}\right) G_{0}^{+}\left(k+q, \tilde{\omega}_{n}+\omega_{m}\right) \\
U_{4} & \equiv U_{4}(0,0)=\frac{1}{\beta L} \sum_{k, \tilde{\omega}_{n}} \frac{1}{\left[i \tilde{\omega}_{n}+v_{F} k\right]^{2}\left[i \tilde{\omega}_{n}-v_{F} k\right]^{2}}
\end{aligned}
$$

The prime on the last sum in Eq. (1.34) denotes that the sums over the $q_{i}$ and $\omega_{m_{i}}$ are restricted to $\sum_{i=1}^{4} q_{i}=0$ and $\sum_{i=1}^{4} \omega_{m_{i}}=0$. Note that a closed-loop theorem assures that all terms in $V$ beyond the quadratic term cancel (see for example [50]). Since in the following we will only consider the leading term of $U_{4}\left(q_{i}, \omega_{m_{i}}\right)$, here we have only

\footnotetext{
${ }^{2}$ Since there is no quartic term in $V$ [see remark below Eq. [1.37], the $V$-field may formally be easily integrated out leading to renormalizations of $\Pi_{0}^{2 k_{F}}\left(q, \omega_{m}\right)$ and $U_{4}\left(q_{i}, \omega_{m_{i}}\right)$
} 
given $U_{4} \equiv U_{4}(0,0)$. Defining 3 the renormalized phonon frequencies $\omega_{\text {ren }}\left(q, \omega_{m}\right)$ and $\omega_{\mathrm{ren}}^{2 k_{F}}\left(q, \omega_{m}\right)$ as

$$
\begin{aligned}
\omega_{\mathrm{ren}}^{2}\left(q, \omega_{m}\right) & \equiv \omega_{q}^{2}\left[1-s\left(\left|g_{q}\right|^{2} / \omega_{q}\right) \Pi_{0}\left(q, \omega_{m}\right)\right] \\
\left(\omega_{\mathrm{ren}}^{2 k_{F}}\left(q, \omega_{m}\right)\right)^{2} & \equiv \omega_{2 k_{F}+q}^{2}\left[1-c s\left(\left|g_{2 k_{F}+q}\right|^{2} / \omega_{2 k_{F}+q}\right) \Pi_{0}^{2 k_{F}}\left(q, \omega_{m}\right)\right],
\end{aligned}
$$

the total (bosonic) action may be written as

$$
\begin{aligned}
S\left\{V, \Delta, \Delta^{*}\right\} & =S\{V\}+S\left\{\Delta, \Delta^{*}\right\}, \\
S\{V\} & =\frac{1}{2} \beta L \sum_{q, \omega_{m}} \frac{1}{\left|g_{q}\right|^{2}}\left[\frac{\omega_{m}^{2}+\omega_{\text {ren }}^{2}\left(q, \omega_{m}\right)}{\omega_{q}}\right] V_{q, \omega_{m}}^{*} V_{q, \omega_{m}}, \\
S\left\{\Delta, \Delta^{*}\right\} & =\frac{1}{c} \beta L \sum_{q, \omega_{m}} \frac{1}{\left|g_{2 k_{F}+q}\right|^{2}}\left[\frac{\omega_{m}^{2}+\left(\omega_{\mathrm{ren}}^{2 k_{F}}\left(q, \omega_{m}\right)\right)^{2}}{\omega_{2 k_{F}+q}}\right] \Delta_{q, \omega_{m}}^{*} \Delta_{q, \omega_{m}} \\
& +\frac{s}{2} \beta L \sum_{q_{i}, \omega_{m_{i}}} U_{4}\left(q_{i}, \omega_{m_{i}}\right) \Delta_{-q_{4},-\omega_{m_{4}}}^{*} \Delta_{-q_{3},-\omega_{m_{3}}}^{*} \Delta_{q_{2}, \omega_{m_{2}}} \Delta_{q_{1}, \omega_{m_{1}}} .
\end{aligned}
$$

\subsubsection{Lindhard function}

The density-density Lindhard response function for a linearised energy dispersion and momentum transfers close to 0 or $2 k_{F}$ is given by $\Pi_{0}\left(q, \omega_{m}\right)$ and $\Pi_{0}^{2 k_{F}}\left(q, \omega_{m}\right)$, respectively. Returning to a quadratic energy dispersion $\varepsilon_{k}=k^{2} / 2 m$, the static Lindhard function per spin direction is given by

$$
\begin{aligned}
\bar{\Pi}_{0}\left(q, \omega_{m}\right) & =-\frac{1}{\beta L} \sum_{k, \tilde{\omega}_{n}} G_{0}\left(k, \tilde{\omega}_{n}\right) G_{0}\left(k+q, \tilde{\omega}_{n}+\omega_{m}\right) \\
& =-\int_{-k_{0}}^{k_{0}} \frac{d k}{2 \pi} \frac{f\left(\varepsilon_{k+q / 2}\right)-f\left(\varepsilon_{k-q / 2}\right)}{k q / m}
\end{aligned}
$$

Here, $G_{0}\left(k, \tilde{\omega}_{n}\right)=\left[i \tilde{\omega}_{n}-\varepsilon_{k}+\mu(T)\right]^{-1}, k_{0}$ is an ultraviolet momentum cutoff,

$$
f\left(\varepsilon_{k}\right)=\frac{1}{\exp \left[\beta\left(\varepsilon_{k}-\mu(T)\right)\right]+1}
$$

is the Fermi function and

$$
\mu(T) \approx \varepsilon_{F}\left(1+\left(\pi^{2} / 12\right)\left(k_{B} T / \varepsilon_{F}\right)^{2}\right)
$$

is the temperature-dependent chemical potential. A numerical evaluation of the static Lindhard function for various temperatures is shown in Fig. 1.1. As the temperature is

\footnotetext{
${ }^{3}$ These frequencies occur naturally in a dynamic theory based on linear response theory (see for example [37]).
} 


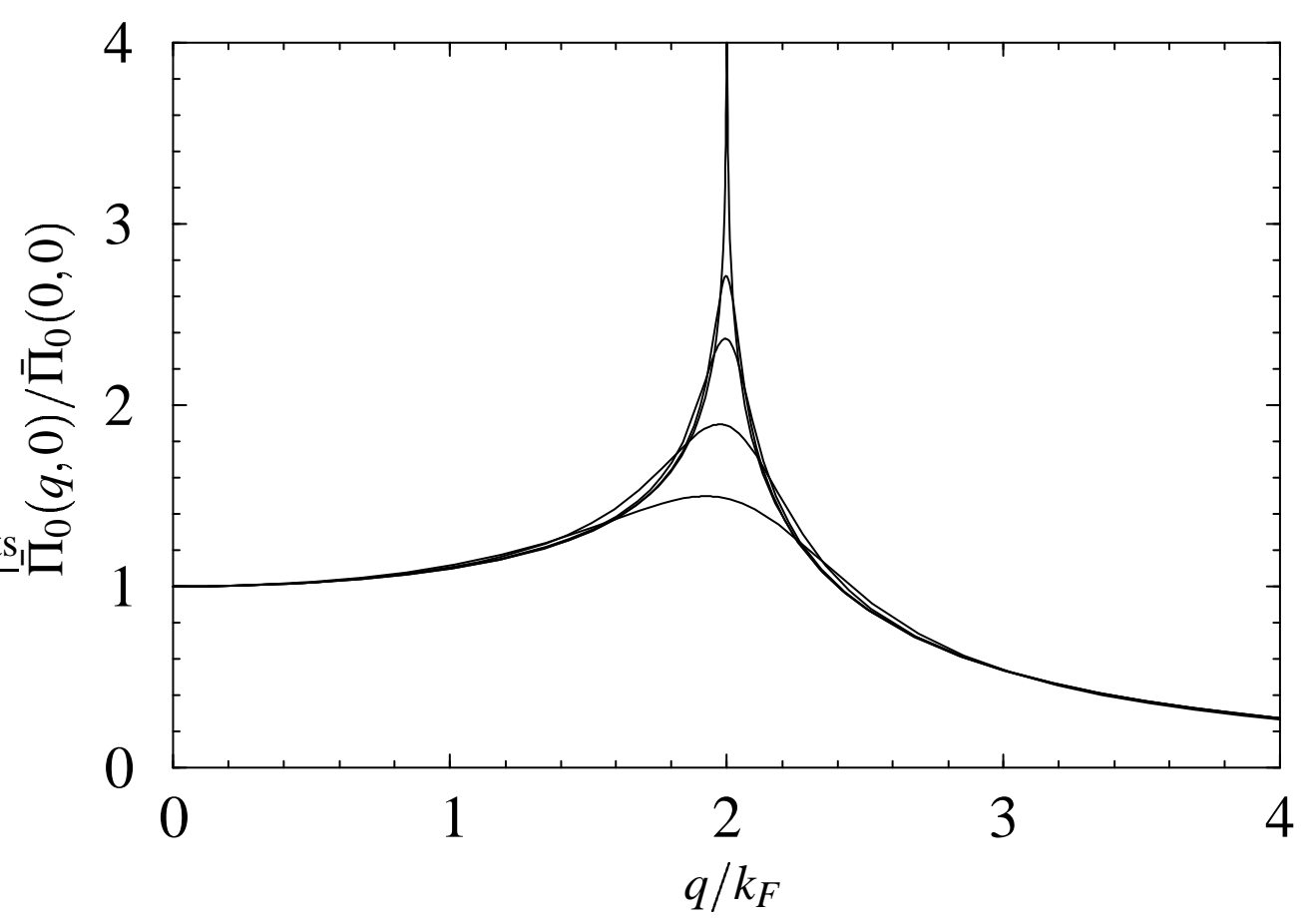

Figure 1.1: The static Lindhard response function $\bar{\Pi}_{0}(q)$ for free electrons with energy dispersion $\varepsilon_{k}=k^{2} / 2 m$ and a momentum cutoff $k_{0}=5 k_{F}$ plotted for the temperatures $k_{B} T / \varepsilon_{F}=0.0,0.01,0.02,0.05$ and 0.1 . As the temperature approaches zero, the Lindhard function clearly diverges at $q=2 k_{F}$. Since $\bar{\Pi}_{0}(q)$ is symmetric with respect to $q \rightarrow-q$, we have only plotted $\bar{\Pi}_{0}(q)$ for positive $q$.

lowered, perfect nesting leads to a singularity at $q=2 k_{F}$.

In the limit $T \rightarrow 0$, the Fermi function renders into a step function such that the integral in 1.43) can be done analytically, resulting in

$$
\bar{\Pi}_{0}(q, 0)=\rho_{0} \frac{k_{F}}{|q|} \ln \left|\frac{2 k_{F}+|q|}{2 k_{F}-|q|}\right|,
$$

where

$$
\rho_{0}=\frac{1}{\pi v_{F}}
$$

is the density of states (DOS) of free Fermions at the Fermi energy per spin direction. While for small $q$ we recover the Thomas-Fermi result $\bar{\Pi}_{0}(q, 0)=\rho_{0}$, for $|q| \approx 2 k_{F}$ we find

$$
\bar{\Pi}_{0}(q, 0)=\frac{\rho_{0}}{2} \ln \left|\frac{2 k_{F}+|q|}{2 k_{F}-|q|}\right|, \quad|| q\left|-2 k_{F}\right| \ll k_{F} .
$$

The divergence of $\bar{\Pi}_{0}(q, 0)$ at $q=2 k_{F}$ can clearly be seen in Fig. 1.1 


\subsubsection{Kohn anomaly in the phonon dispersion}

The singularity in the (static) Lindhard response function has a dramatic effect on the renormalized phonon dispersion. In the commensurate case, Eqs. (1.38) and (1.39) can be generalized towards arbitrary momenta $q$ by defining [75]

$$
\bar{\omega}_{\mathrm{ren}}^{2}\left(q, \omega_{m}\right) \equiv \omega_{q}^{2}\left[1-s\left(\left|g_{q}\right|^{2} / \omega_{q}\right) \bar{\Pi}_{0}\left(q, \omega_{m}\right)\right]
$$

where $\bar{\Pi}_{0}\left(q, \omega_{m}\right)$ was introduced in the last subsection. For small $q$ the coupling constant $g_{q}$ varies as $q^{1 / 2}$ (see Ref. [37]) such that $\left|g_{q}\right|^{2} / \omega_{q}$ is independent of $q$. In one dimension it should be not such a bad approximation to assume the dimensionless coupling constant

$$
\lambda \equiv s \frac{\rho_{0}\left|g_{q}\right|^{2}}{2 \omega_{q}}
$$

to be independent of $q$. A plot of the bare acoustic phonon dispersion $\omega_{q}=$ $2 \omega_{0} \sin |q a / \pi|$ and the renormalized phonon dispersion

$$
\bar{\omega}_{\text {ren }}\left(q, \omega_{m}\right) \equiv \omega_{q}\left[1-2 \lambda \bar{\Pi}_{0}\left(q, \omega_{m}\right) / \rho_{0}\right]^{1 / 2}
$$

for different temperatures is shown in Fig. 1.2 The singularity in the Lindhard function is clearly conveyed into a singularity in the renormalized phonon dispersion and is known as a Kohn anomaly [47]. As the temperature is lowered, $\bar{\omega}_{\text {ren }}\left(2 k_{F}\right)$ decreases all the way down to zero, leading to a finite transition temperature $T_{c}^{\mathrm{MF}}$ at which $\bar{\omega}_{\text {ren }}\left(2 k_{F}\right)$ becomes zero. At $T_{c}^{\mathrm{MF}}$, the $q=2 k_{F}$ phonon mode softens, i.e. becomes gapless. We will see shortly that this dynamic definition of $T_{c}^{\mathrm{MF}}$ agrees with a thermodynamic definition of the mean-field transition temperature $T_{c}^{\mathrm{MF}}$. In the thermodynamic approach, the free energy will be minimized for a finite $\Delta$ below $T_{c}^{\mathrm{MF}}$.

The case of a half-filled band behaves slightly different. In this case the two singularities at $\pm 2 k_{F}$ merge at the boundary of the first Brillouin zone. The general picture of the softening of the phonon mode, however, is the same.

\subsubsection{Discarding quantum fluctuations}

To derive a time-independent Ginzburg-Landau theory we ignore quantum fluctuations, i.e. we ignore all terms involving finite bosonic frequencies. Since we will be only interested in static properties this should be a reasonable approximation for not too small temperatures. The action $S\left\{V, \Delta, \Delta^{*}\right\}$ then turns into the free energy functional $\beta F\left\{V, \Delta, \Delta^{*}\right\}$, where

$$
\begin{aligned}
F\left\{V, \Delta, \Delta^{*}\right\} & =F\{V\}+F\left\{\Delta, \Delta^{*}\right\} \\
F\{V\} & =\frac{1}{2} L \sum_{q} \frac{1}{\left|g_{q}\right|^{2}}\left[\frac{\omega_{\text {ren }}^{2}(q)}{2 \omega_{q}}\right] V_{q}^{*} V_{q}
\end{aligned}
$$




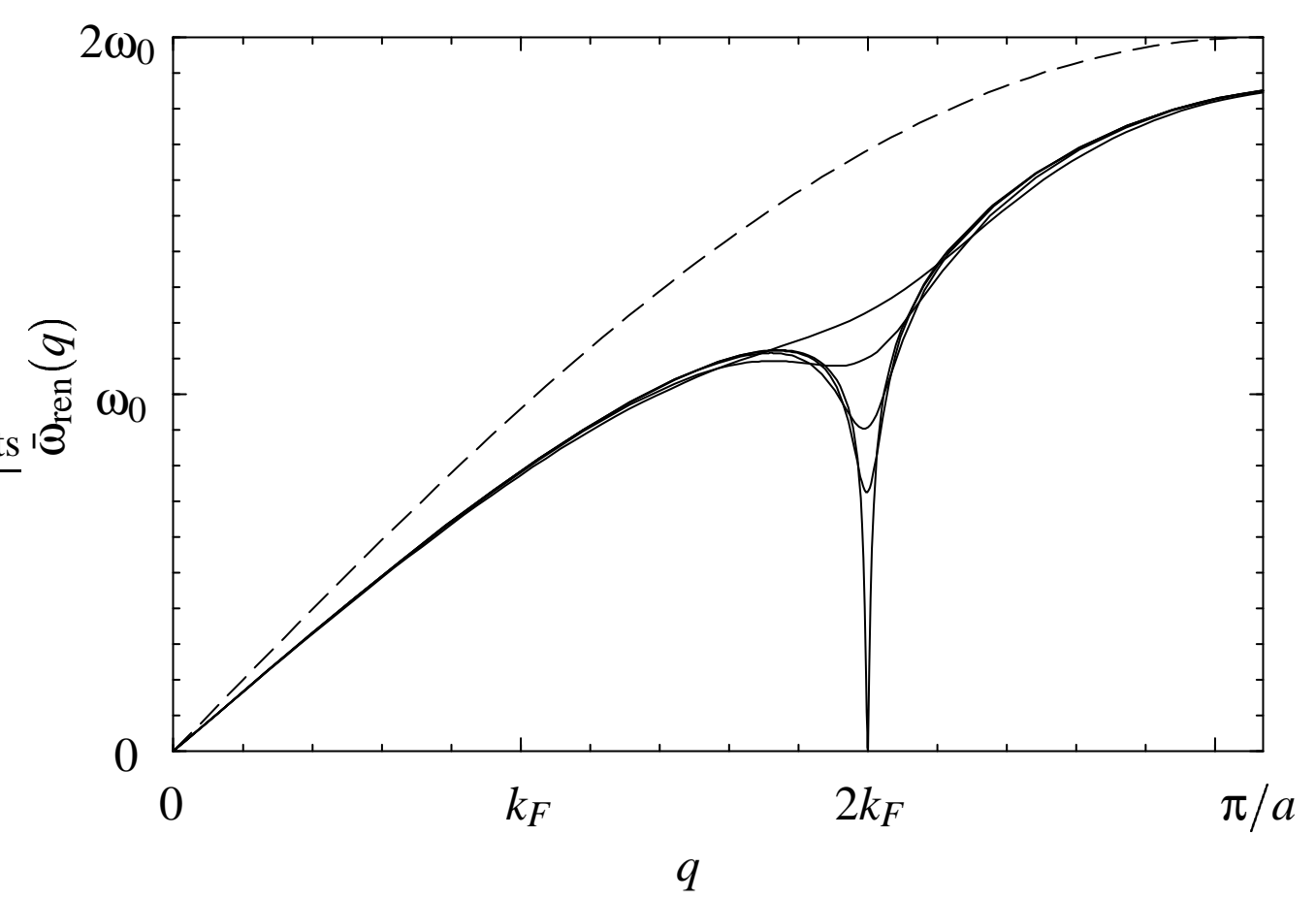

Figure 1.2: Plot of the acoustic phonon dispersion $\omega_{q}=2 \omega_{0} \sin |q a / \pi|$ (dashed line) and the respective renormalized phonon dispersion $\bar{\omega}_{\text {ren }}\left(q, \omega_{m}\right)=$ $\omega_{q}\left(1-\lambda \bar{\Pi}_{0}\left(q, \omega_{m}\right) / \rho_{0}\right)^{1 / 2}$ for $\lambda=0.15$ and $k_{B} T / \varepsilon_{F}=0.1,0.05,0.02,0.01$ and $T=$ $T_{c}^{\mathrm{MF}}$.

$$
\begin{aligned}
F\left\{\Delta, \Delta^{*}\right\} & =\frac{1}{c} L \sum_{q} \frac{1}{\left|g_{2 k_{F}+q}\right|^{2}}\left[\frac{\left(\omega_{\text {ren }}^{2 k_{F}}(q)\right)^{2}}{\omega_{2 k_{F}+q}}\right] \Delta_{q}^{*} \Delta_{q} \\
& +\frac{s}{2} L \sum_{q_{i}}^{\prime} U_{4}\left(q_{i}\right) \Delta_{-q_{4}}^{*} \Delta_{-q_{3}}^{*} \Delta_{q_{2}} \Delta_{q_{1}} .
\end{aligned}
$$

It is not difficult to show that

$$
\Pi_{0}\left(q, \omega_{m}\right)=\rho_{0} \frac{v_{F}^{2} q^{2}}{\omega_{m}^{2}+v_{F}^{2} q^{2}},
$$

which implies that for a linearized energy dispersion $\Pi_{0}(q, 0)=\rho_{0}$ is even valid for arbitrary temperatures. As we have already seen in Fig. 1.2, the Lindhard function therefore only leads to a small modification of the acoustic phonon dispersion for small $q$. Since $F\{V\}$ is Gaussian in $V$, the electron-phonon coupling only leads to slightly renormalized phonon statistics for small $q$.

Let us now focus on the truncated free energy functional $F\left\{\Delta, \Delta^{*}\right\}$ : Expanding 
$\Pi_{0}^{2 k_{F}}(q) \equiv \Pi_{0}^{2 k_{F}}(q, 0)$ up to terms of second order in $q$, we have

$$
\Pi_{0}^{2 k_{F}}(q)=\Pi_{0}^{2 k_{F}}(0)-C v_{F}^{2} q^{2}
$$

with

$$
C=\frac{1}{\beta L} \sum_{k, \tilde{\omega}_{n}} \frac{1}{\left[i \tilde{\omega}_{n}+v_{F} k\right]\left[i \tilde{\omega}_{n}-v_{F} k\right]^{3}} .
$$

$\Pi_{0}^{2 k_{F}}(0)$ may be calculated as follows:

$$
\begin{aligned}
\Pi_{0}^{2 k_{F}}(0) & =-\frac{1}{\beta L} \sum_{k, \tilde{\omega}_{n}} G_{0}^{-}\left(k, \tilde{\omega}_{n}\right) G_{0}^{+}\left(k, \tilde{\omega}_{n}\right) \\
& =-\int_{-k_{0}}^{k_{0}} \frac{d k}{2 \pi} \frac{f\left(v_{F} k\right)-f\left(-v_{F} k\right)}{2 v_{F} k} \\
& =\int_{-k_{0}}^{k_{0}} \frac{d k}{2 \pi} \frac{\tanh \left(\beta v_{F} k / 2\right)}{2 v_{F} k} \\
& =\frac{\rho_{0}}{2} \int_{0}^{\varepsilon_{0} / 2 k_{B} T} d u \frac{\tanh u}{u}
\end{aligned}
$$

where the momentum cutoff $k_{0}$ turns into the energy cutoff $\varepsilon_{0} \equiv v_{F} k_{0}$ and due to the linearization of the energy dispersion, the chemical potential in the above Fermi function equals zero. For $\varepsilon_{0} / k_{B} T \gg 1$, the last integral in Eq. (1.58) depends logarithmically on $\varepsilon_{0} / 2 k_{B} T$. The additive constant can be obtained by partially integrating the last integral in Eq. (1.58) and then looking up the remaining integral (Eq. (4.371) in Ref. [35]):

$$
\int_{0}^{x} d u \frac{\tanh u}{u}=\ln (x)-\int_{0}^{\infty} d u \frac{\ln u}{\cosh ^{2} u}+O\left(e^{-x}\right)=\ln \left(4 e^{\gamma} x / \pi\right)+O\left(e^{-x}\right),
$$

where

$$
\gamma \equiv \lim _{N \rightarrow \infty}\left(\sum_{n=1}^{N} \frac{1}{n}-\ln (N)\right)=0.577215664 \ldots .
$$

is Euler's constant. We therefore find for $\Pi_{0}^{2 k_{F}}(0)$

$$
\Pi_{0}^{2 k_{F}}(0)=\rho_{0} / 2 \ln \left[\left(2 e^{\gamma} / \pi\right) \varepsilon_{0} / k_{B} T\right]
$$

with 4

$$
\frac{2 e^{\gamma}}{\pi}=1.1338659 \ldots
$$

The mean-field transition temperature may now be obtained by plugging Eq. (1.61) into Eq. (1.39) and setting the renormalized phonon dispersion equal to zero. It directly follows

$$
k_{B} T_{c}^{\mathrm{MF}}=1.134 \varepsilon_{0} \exp (-1 / \lambda),
$$

${ }^{4}$ Note that in the literature on the BCS-theory of superconductivity or the Peierls instability, many people use the value 1.14 instead of 1.134. Of course, this slight deviation does not make any difference. 
where

$$
\lambda \equiv c s \frac{\rho_{0}\left|g_{q}\right|^{2}}{2 \omega_{q}}
$$

is the dimensionless coupling constant already considered above. In the weak-coupling limit we have (by definition) $\lambda \ll 1$.

To calculate $C$ and $U_{4}$, let us consider the general expression

$$
C_{v_{1}, v_{2}}=\frac{1}{\beta L} \sum_{k, \tilde{\omega}_{n}} \frac{1}{\left[i \tilde{\omega}_{n}+v_{F} k\right]^{v_{1}}\left[i \tilde{\omega}_{n}-v_{F} k\right]^{v_{2}}}
$$

which clearly vanishes if $v_{1}+v_{2}$ is odd and greater than two. Turning the $k$-summation into an integral and using the residue theorem, it follows for even $v \equiv v_{1}+v_{2} \geq 4$

$$
\begin{aligned}
C_{v_{1}, v_{2}} & =\frac{2}{\beta} \sum_{\tilde{\omega}_{n}>0} \int \frac{d u}{2 \pi v_{F}} \frac{1}{\left[i \tilde{\omega}_{n}+u\right]^{v_{1}}\left[i \tilde{\omega}_{n}-u\right]^{v_{2}}} \\
& =-\left.\frac{\rho_{0}}{2} \frac{4 \pi i}{\beta} \sum_{\tilde{\omega}_{n}>0} \frac{1}{\left(v_{1}-1\right) !}\left(\frac{d}{d u}\right)^{v_{1}-1} \frac{1}{\left[i \tilde{\omega}_{n}-u\right]^{v_{2}}}\right|_{u=-i \tilde{\omega}_{n}} \\
& =-\frac{\rho_{0}}{2} \frac{4 \pi i}{\beta} \frac{(v-2)(v-3) \cdots v_{2}}{\left(v_{1}-1\right) !} \sum_{\tilde{\omega}_{n}>0} \frac{1}{\left[2 i \tilde{\omega}_{n}\right]^{v-1}} \\
& =\frac{\rho_{0}}{2}(-1)^{v / 2}\left(\frac{\beta}{4 \pi}\right)^{v-2}\left(\begin{array}{c}
v-2 \\
v_{1}-1
\end{array}\right)\left(2^{v-1}-1\right) \zeta(v-1),
\end{aligned}
$$

where in the last step we have used

$$
\begin{aligned}
\sum_{\tilde{\omega}_{n}>0} \frac{1}{\left[2 i \tilde{\omega}_{n}\right]^{v-1}} & =\left(\frac{\beta}{2 \pi i}\right)^{v-1} \sum_{n \text { odd }} \frac{1}{n^{v-1}} \\
& =\left(\frac{\beta}{2 \pi i}\right)^{v-1}\left(\sum_{n=1}^{\infty} \frac{1}{n^{v-1}}-\sum_{n=1}^{\infty} \frac{1}{(2 n)^{v-1}}\right) \\
& =\left(\frac{\beta}{4 \pi i}\right)^{v-1}\left(2^{v-1}-1\right) \zeta(v-1) .
\end{aligned}
$$

The Riemann zeta-function which appears here is defined as

$$
\zeta(v) \equiv \sum_{n=1}^{\infty} \frac{1}{n^{v}} .
$$

It directly follows from Eq. (1.66) that

$$
U_{4}=2 C=\rho_{0}\left(\frac{\beta}{4 \pi}\right)^{2} 7 \zeta(3) .
$$




\subsubsection{Ginzburg-Landau functional}

With the above approximations, the truncated free energy functional turns into

$$
\begin{aligned}
F\left\{\Delta, \Delta^{*}\right\}=\frac{s \rho_{0} L}{2}\left[a(T) \sum_{q} \Delta_{q}^{*} \Delta_{q}+b(T) \sum_{q_{i}}^{\prime} \Delta_{-q_{4}}^{*} \Delta_{-q_{3}}^{*} \Delta_{q_{2}} \Delta_{q_{1}}\right. & \\
& \left.+c(T) \sum_{q} q^{2} \Delta_{q}^{*} \Delta_{q}\right]
\end{aligned}
$$

where the temperature-dependent coefficients $a(T), b(T)$ and $c(T)$ are given by

$$
\begin{aligned}
& a(T)=\ln \frac{T}{T_{c}^{\mathrm{MF}}}, \quad k_{B} T_{c}^{\mathrm{MF}}=1.134 \varepsilon_{0} \exp (-1 / \lambda), \\
& b(T)=\left(\frac{1}{4 \pi k_{B} T}\right)^{2} 7 \zeta(3) . \\
& c(T)=\left(\frac{v_{F}}{4 \pi k_{B} T}\right)^{2} 7 \zeta(3) .
\end{aligned}
$$

We now define the order parameter field $\Delta(x)$ as the Fourier transform of $\Delta_{q}$ :

$$
\begin{aligned}
\Delta(x) & =\sum_{q} e^{i q x} \Delta_{q}, \\
\Delta_{q} & =\frac{1}{L} \int_{0}^{L} d x e^{-i q x} \Delta(x) .
\end{aligned}
$$

Note that the order parameter field $\Delta(x)$ and the order parameter $\langle\Delta(x)\rangle$ are real in the commensurate case where $\Delta_{q}^{*}=\Delta_{-q}$. In the incommensurate case, $\Delta_{q}^{*}=\Delta_{-q}$ does not hold and $\Delta(x)$ and $\langle\Delta(x)\rangle$ are complex. Note also that with the above definitions of the prefactors of the Fourier transform both $\Delta(x)$ and $\Delta_{q}$ have dimensions of energy 5 In terms of $\Delta(x)$ the Ginzburg-Landau functional assumes the usual form

$$
F\left\{\Delta, \Delta^{*}\right\}=\frac{s \rho_{0}}{2} \int_{0}^{L} d x\left[a(T)|\Delta(x)|^{2}+b(T)|\Delta(x)|^{4}+c(T)\left|\partial_{x} \Delta(x)\right|^{2}\right]
$$

In the microscopic derivation of this static Ginzburg-Landau functional we have ignored quantum effects. The resulting free energy functional was expanded up to terms of fourth order in the order parameter field and only the leading term involving gradients in the order parameter field was retained. These approximations can only be good at sufficiently high temperatures provided that the order parameter field and its fluctuations are small. In particular, we have to satisfy the condition $|\Delta| \ll k_{B} T$. To weaken the above restrictions, higher terms in the order parameter field have to be included.

\footnotetext{
${ }^{5}$ We have introduced $\Delta_{q}$ such that below a phase transition $\Delta_{q=0}$ assumes the finite value $\Delta_{0} e^{i \vartheta}$. Other definitions are, of course, also possible.
} 
A method based on the formalism developed in the next chapter which allows to expand the free energy functional in terms of gradients of the order parameter field but in each order collects all terms in the order parameter itself is presented in Appendix A Up to terms of second order in the gradient, we get the generalized Ginzburg-Landau functional

$$
\begin{aligned}
F\left\{\Delta, \Delta^{*}\right\} & =F^{(0)}\left\{\Delta, \Delta^{*}\right\}+F^{(2)}\left\{\Delta, \Delta^{*}\right\}, \\
F^{(0)}\left\{\Delta, \Delta^{*}\right\} & =s \rho_{0} \int_{0}^{L} d x\left(-\frac{2 \pi}{\beta} \sum_{0<\tilde{\omega}_{n} \leq \varepsilon_{0}}\left[\sqrt{\tilde{\omega}_{n}^{2}+|\Delta|^{2}}-\tilde{\omega}_{n}\right]+\frac{|\Delta|^{2}}{2 \lambda}\right), \\
F^{(2)}\left\{\Delta, \Delta^{*}\right\} & =s \rho_{0} \int_{0}^{L} d x \frac{2 \pi}{\beta} \sum_{\tilde{\omega}_{n}>0}\left[\frac{1}{8} \frac{\left|\partial_{x} \Delta\right|^{2}}{\left(\tilde{\omega}_{n}^{2}+|\Delta|^{2}\right)^{\frac{3}{2}}}-\frac{1}{32} \frac{\left[\partial_{x}|\Delta|^{2}\right]^{2}}{\left(\tilde{\omega}_{n}^{2}+|\Delta|^{2}\right)^{\frac{5}{2}}}\right] .
\end{aligned}
$$

For $F^{(0)}\left\{\Delta, \Delta^{*}\right\}$ to be finite and to avoid logarithmic divergences, the sum in Eq. (1.79) needs to be regularized by an ultraviolet cutoff $\varepsilon_{0} 6$ Although the ultraviolet cutoff $\varepsilon_{0}$ was introduced here in the sum over Matsubara frequencies instead of as a cutoff in the momentum integral, expanding Eqs. (1.77) to (1.79) in the regime $\beta|\Delta| \ll 1$ and using the definition of Euler's constant given in Eq. (1.60) to evaluate the remaining sum in $F^{(0)}\left(\Delta, \Delta^{*}\right)$, we recover precisely the Ginzburg-Landau functional (1.76).

\subsection{Mean-field theory}

As a first step to understand the phenomenon of the Peierls transition, we consider our system in the mean field approximation, i.e. we ignore fluctuations of the order parameter field $\Delta(x)$ and consider $\Delta$ to be spatially constant. Since the gradient term vanishes, the generalized Ginzburg-Landau functional 1.77) reduces to

$$
F(\Delta)=s \rho_{0} L\left(-\frac{2 \pi}{\beta} \sum_{0<\tilde{\omega}_{n} \lesssim \varepsilon_{0}}\left[\sqrt{\tilde{\omega}_{n}^{2}+|\Delta|^{2}}-\tilde{\omega}_{n}\right]+\frac{|\Delta|^{2}}{2 \lambda}\right) .
$$

A plot of $F(\Delta)$ for different temperatures $T$ and real $\Delta$ is shown in Fig. 1.3. As long as $T>T_{c}^{\mathrm{MF}}$, the generalized Landau function (1.80) assumes its minimum at $\Delta=0$. At $T<T_{c}^{\mathrm{MF}}$, however, the coefficient of the quadratic term in the expansion of the generalized Landau function, $\left(s \rho_{0} / 2\right) a(T)$, becomes negative, and $F(\Delta)$ takes on its minima at the finite values $\Delta=\Delta_{0} e^{i \vartheta}$ which satisfy $|\Delta|=\Delta_{0} \neq 0$. While $\vartheta$ is an arbitrary but constant phase in the incommensurate case, it is only allowed to assume the values 0 and $\pi$ in the commensurate case, such that $\Delta=\Delta_{0} e^{i \vartheta}= \pm \Delta_{0}$ is real. As long as $T$ is close to the mean-field critical temperature $T_{c}^{\mathrm{MF}}, \Delta_{0} / k_{B} T$ is small and the

${ }^{6}$ This cutoff has to be introduced such that $F^{(0)}\left\{\Delta, \Delta^{*}\right\}$ is a smooth function of temperature. One possibility to do so is to weigh the last summand with a factor between 0 and 1 depending on the difference between its frequency $\tilde{\omega}_{n}$ and $\varepsilon_{0}$. 


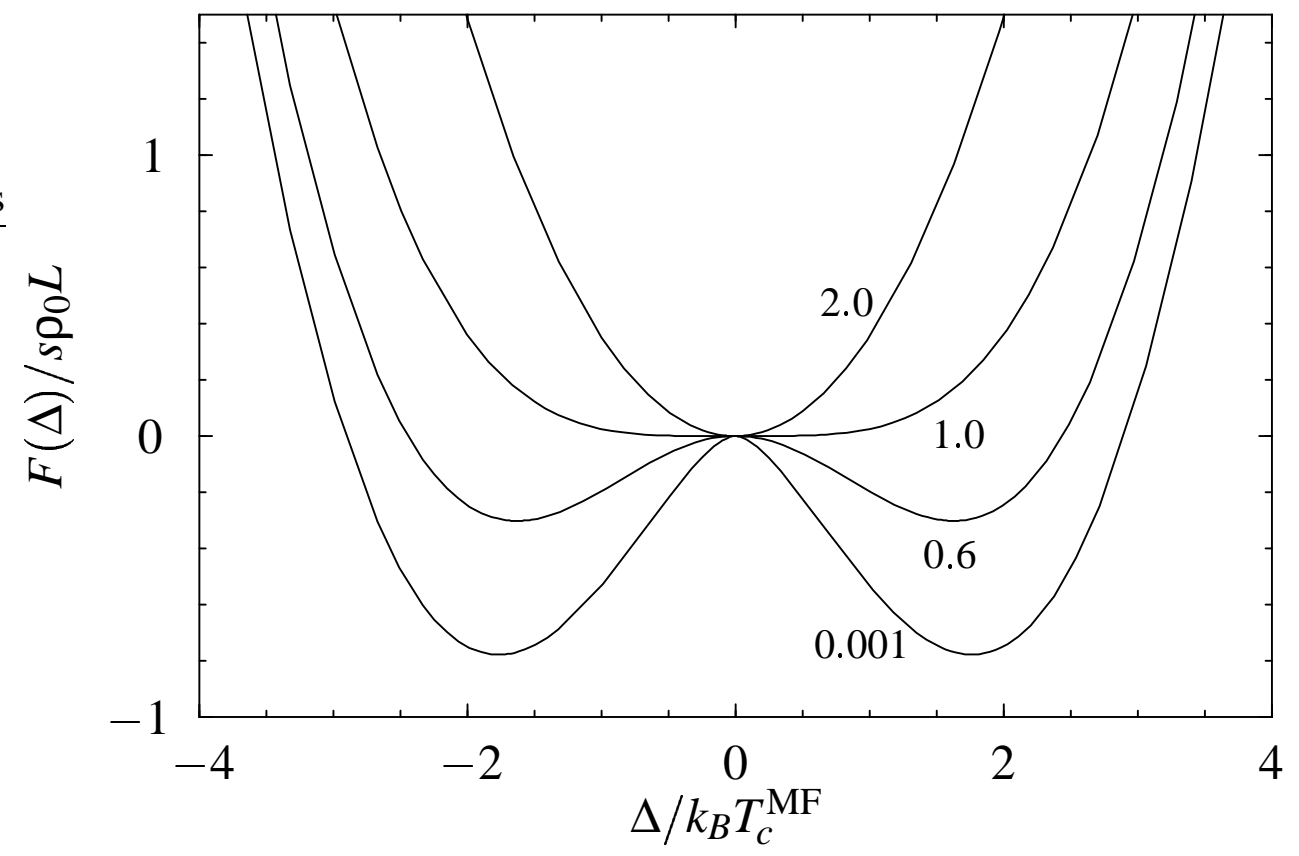

Figure 1.3: Plot of the generalized Landau function $F(\Delta)$ [see Eq. (1.80)] as a function of the real variable $\Delta$ for $T / T_{c}^{\mathrm{MF}}=0.001,0.6,1.0$, and 2.0. For $T<T_{c}^{\mathrm{MF}}$, the Landau function $F(\Delta)$ takes on its minima at the finite values $\pm \Delta_{0}(T) \neq 0$.

Landau function obtained from Eq. (1.76) by setting $\Delta(x)=\Delta$ is a good approximation to its generalized form. In this case, $F(\Delta)$ is minimized for $\Delta=\Delta_{0}(T) e^{i \vartheta}$, where

$$
\Delta_{0}(T)=\left(\frac{|a(T)|}{2 b(T)}\right)^{1 / 2}, \quad T \lesssim T_{c}^{\mathrm{MF}}
$$

Since $T \lesssim T_{c}^{\mathrm{MF}}$, we may replace $a(T)$ by $\left(T-T_{c}^{\mathrm{MF}}\right) / T_{c}^{\mathrm{MF}}$ and $b(T)$ by $b\left(T_{c}^{\mathrm{MF}}\right)$, such that

$$
\Delta_{0}(T)=\frac{4 \pi k_{B} T_{c}^{\mathrm{MF}}}{(2 \cdot 7 \zeta(3))^{1 / 2}}\left(\frac{T_{c}^{\mathrm{MF}}-T}{T_{c}^{\mathrm{MF}}}\right)^{1 / 2}, \quad T \lesssim T_{c}^{\mathrm{MF}} .
$$

Note that we have defined $\Delta_{0}(T)$ such that it is real and positive. The critical exponent $\bar{\beta}=1 / 2$ is the typical mean-field exponent. Since $\Delta_{0}(T)$ rises continuously from zero at $T=T_{c}^{\mathrm{MF}}$, the system undergoes a second order phase transition. For arbitrary temperatures, $\Delta_{0}(T)$ may be calculated by minimizing Eq. (1.80). In this case, $\Delta_{0}(T)$ is implicitly given by the BCS gap equation [1, 83, 90]

$$
\frac{1}{\lambda}=\frac{2 \pi}{\beta} \sum_{0<\tilde{\omega}_{n} \lesssim \varepsilon_{0}} \frac{1}{\sqrt{\tilde{\omega}_{n}^{2}+\Delta_{0}^{2}(T)}},
$$




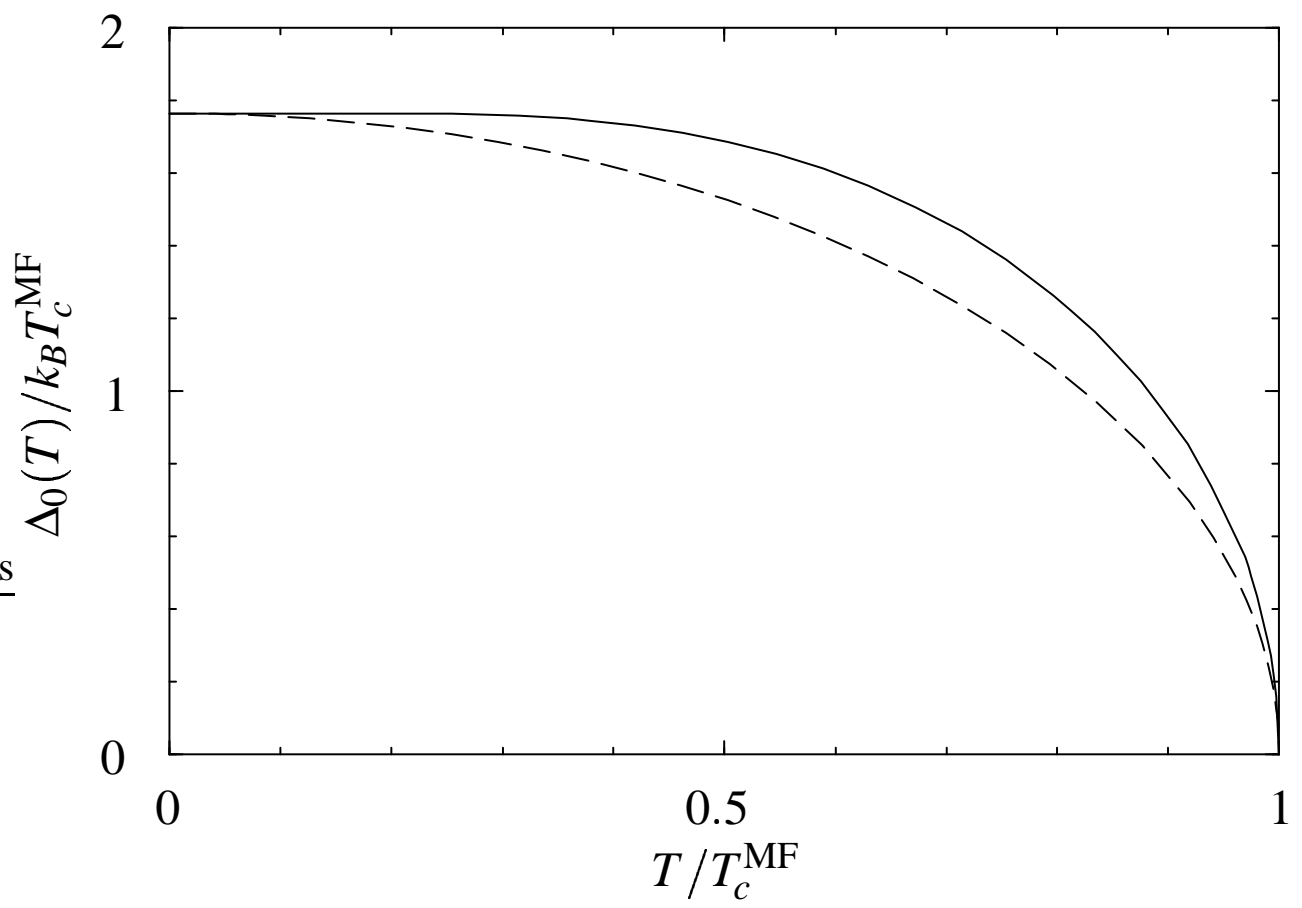

Figure 1.4: The solid line shows a plot of the energy gap $\Delta_{0}(T)$ as a function of temperature. For a comparison we also plot as a dashed line the usual BCS-approximation $\Delta_{0}(T)=1.76 k_{B} T_{c}^{\mathrm{MF}}\left(1-\left(T / T_{c}^{\mathrm{MF}}\right)^{2}\right)^{1 / 2}$.

which can also be written as

$$
\frac{1}{\lambda}=\int_{0}^{\varepsilon_{0}} d \varepsilon \frac{\tanh \left[\left(\varepsilon^{2}+\Delta_{0}^{2}(T)\right)^{1 / 2} / 2 k_{B} T\right]}{\left(\varepsilon^{2}+\Delta_{0}^{2}(T)\right)^{1 / 2}} .
$$

For $T=T_{c}^{\mathrm{MF}}$, we have $\Delta_{0}(T)=0$, and using Eq. (1.59) we recover Eq. (1.63). A plot of $\Delta_{0}(T)$ obtained by a numerical solution of Eq. (1.83) is shown in Fig. 1.4.

At zero temperature, $F(\Delta)$ and $\Delta_{0}(0)$ can be simplified as follows: In the limit $\beta \rightarrow \infty$, the sum over Matsubara frequencies in Eq. (1.80) turns into an integral,

$$
F_{T=0}(\Delta)=s \rho_{0} L\left(-\int_{0}^{\varepsilon_{0}} d E\left[\sqrt{E^{2}+|\Delta|^{2}}-E\right]+\frac{|\Delta|^{2}}{2 \lambda}\right) .
$$

The integral may be done analytically, resulting in

$$
F_{T=0}(\Delta)=\frac{s \rho_{0}}{2} L\left(-\varepsilon_{0} \sqrt{\varepsilon_{0}^{2}+|\Delta|^{2}}+\varepsilon_{0}^{2}-|\Delta|^{2} \ln \frac{\varepsilon_{0}+\sqrt{\varepsilon_{0}^{2}+|\Delta|^{2}}}{|\Delta|}+\frac{|\Delta|^{2}}{\lambda}\right)
$$


To be consistent with our weak-coupling assumption which implies $|\Delta| \ll \varepsilon_{0}$, we ignore terms of order $|\Delta|^{2} / \varepsilon_{0}^{2}$, such that

$$
F_{T=0}(\Delta)=-\frac{s \rho_{0}}{2} L|\Delta|^{2}\left(\frac{1}{2}+\ln \left(\frac{2 \varepsilon_{0}}{|\Delta|}\right)-\frac{1}{\lambda}\right)
$$

Setting the derivative of this equation with respect to $\Delta$ equal to zero, we obtain for the minimized free energy

$$
F_{T=0}\left(\Delta_{0}(0)\right)=-\frac{s \rho_{0} L}{2} \frac{\Delta_{0}^{2}(0)}{2}
$$

$\Delta_{0}(0)$, which we have chosen again real and positive, can be obtained in the weakcoupling limit $\lambda \ll 1$ :

$$
\Delta_{0}(0)=2 \varepsilon_{0} e^{-1 / \lambda}
$$

Expressing $\lambda$ in terms of the mean-field transition temperature $T_{c}^{\mathrm{MF}}$, we obtain the usual BCS-relation between the zero-temperature gap and the mean-field transition temperature,

$$
2 \Delta_{0}(0)=3.528 k_{B} T_{c}^{\mathrm{MF}}
$$

\subsubsection{Static lattice distortion}

It directly follows from Eq. (1.6) that the expectation value of the lattice displacements is given by

$$
\left\langle u\left(x_{n}\right)\right\rangle=\sum_{q} e^{i q x_{n}}\left(\frac{1}{2 N M \omega_{q}}\right)^{1 / 2}\left(\left\langle b_{q}\right\rangle+\left\langle b_{-q}^{\dagger}\right\rangle\right) .
$$

In the mean-field approximation, the only non-vanishing contributions are

$$
\langle\Delta(x)\rangle=\Delta_{0} e^{i \vartheta}=\frac{\left|g_{2 k_{F}}\right|}{\sqrt{L}}\left(\left\langle b_{2 k_{F}}\right\rangle+\left\langle b_{-2 k_{F}}^{\dagger}\right\rangle\right)
$$

and its conjugated form. We therefore have

$$
\left\langle u\left(x_{n}\right)\right\rangle=\left(\frac{2 a}{M \omega_{2 k_{F}}\left|g_{2 k_{F}}\right|^{2}}\right)^{1 / 2} \Delta_{0} \cos \left(2 k_{F} x_{n}+\vartheta\right) .
$$

Below the Peierls transition $\Delta_{0}>0$, and the system develops a static lattice distortion with wave vector $2 k_{F}$ whose amplitude is proportional to the order parameter $\langle\Delta(x)\rangle=$ $\Delta_{0} e^{i \vartheta}$. In the case of a half-filled band, $k_{F}=\pi / 2 a$, such that all ions are displaced by the same amount, but in alternating directions. 


\subsubsection{Mean-field Hamiltonian}

Treating the phonon field in a mean-field approximation, the electronic part of the action (1.25) corresponds to the Hamiltonian

$$
\mathcal{H}_{\mathrm{el}}^{\mathrm{MF}}=\sum_{k}\left(c_{+, k}^{\dagger}, c_{-, k}^{\dagger}\right) H_{k}\left(\begin{array}{c}
c_{+, k} \\
c_{-, k}
\end{array}\right), \quad H_{k}=\left(\begin{array}{cc}
v_{F} k & \Delta_{0} e^{i \vartheta} \\
\Delta_{0} e^{-i \vartheta} & -v_{F} k
\end{array}\right) .
$$

Here, $c_{ \pm, k}^{\dagger}$ and $c_{ \pm, k}$ are creation and annihilation operators of right- or left-moving electrons with momentum $k$ measured relative to the Fermi energy. For simplicity, we have ignored the spin index. The above Hamiltonian can be diagonalized by the Bogoliubov transformation

$$
\left(\begin{array}{c}
c_{+, k} \\
c_{-, k}
\end{array}\right)=U_{k}\left(\begin{array}{c}
a_{+, k} \\
a_{-, k}
\end{array}\right)
$$

where $U_{k}$ is a $2 \times 2$-matrix. For the new fermion operators $a_{ \pm, k}^{\dagger}$ and $a_{ \pm, k}$ to satisfy the usual anticommutation relations

$$
\left\{a_{\alpha, k}, a_{\alpha^{\prime}, k^{\prime}}\right\}=0, \quad\left\{a_{\alpha, k}, a_{\alpha^{\prime}, k^{\prime}}^{\dagger}\right\}=\delta_{\alpha, \alpha^{\prime}} \delta_{k, k^{\prime}} \mathbf{1}
$$

$U_{k}$ has to be a special unitary matrix, i.e. its matrix elements have to satisfy

$$
\begin{gathered}
\left|U_{++, k}\right|^{2}+\left|U_{+-, k}\right|^{2}=1 \\
U_{--, k}=U_{++, k}^{*} \quad, \quad U_{-+, k}=-U_{+-, k}^{*} .
\end{gathered}
$$

The eigenvalues of $H_{k}$ can easily be shown to be $\pm E_{k}$, where

$$
E_{k}=\operatorname{sgn}(k) \sqrt{v_{F}^{2} k^{2}+\Delta_{0}^{2}} .
$$

Here, we have chosen a sign convention such that $E_{k} \rightarrow v_{F} k$ for $\Delta_{0} / v_{F} k \rightarrow 0$. A straightforward evaluation of the corresponding eigenvectors determines the unitary matrix $U_{k}$ :

$$
U_{k}=\frac{1}{\sqrt{2}}\left(\begin{array}{cc}
\left(1+v_{F} k / E_{k}\right)^{1 / 2} e^{i \vartheta / 2} & -\operatorname{sgn}(k)\left(1-v_{F} k / E_{k}\right)^{1 / 2} e^{i \vartheta / 2} \\
\operatorname{sgn}(k)\left(1-v_{F} k / E_{k}\right)^{1 / 2} e^{-i \vartheta / 2} & \left(1+v_{F} k / E_{k}\right)^{1 / 2} e^{-i \vartheta / 2}
\end{array}\right) .
$$

For $\Delta / v_{F} k \rightarrow 0$ the unitary matrix $U_{k}$ scales to the identity 1 . In terms of the new fermion operators, the electronic part of the mean-field Hamiltonian reads

$$
\mathcal{H}_{\mathrm{el}}^{\mathrm{MF}}=\sum_{\alpha, k} \alpha E_{k} a_{\alpha, k}^{\dagger} a_{\alpha, k}
$$

For finite $\Delta_{0}$ the energy dispersion of this Hamiltonian is gapped. A plot of the energy dispersion $E_{|k|-k_{F}}$ relative to the Fermi energy is shown in Fig. 1.5. 


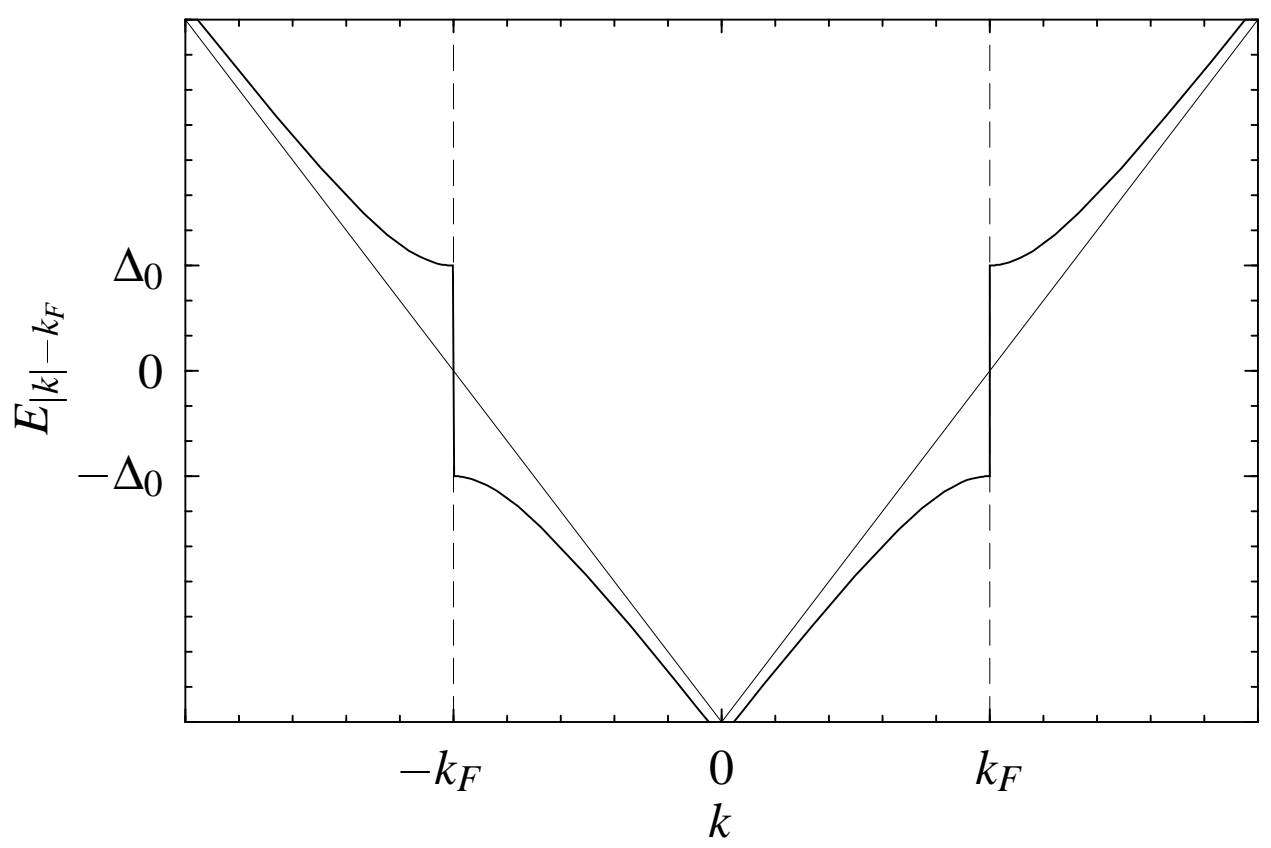

Figure 1.5: Exaggerated plot of the energy dispersion $E_{|k|-k_{F}}$ relative to the Fermi level of the mean-field Hamiltonian. In the weak-coupling limit discussed in the text we should have $\Delta_{0} \ll \varepsilon_{F}$.

\subsubsection{The density of states}

The fundamental quantity which will lie at the heart of the following chapters and which encapsulates the whole thermodynamics is the density of states (DOS). In the mean-field picture discussed here, the DOS $\rho(\omega)$ is given by

$$
\begin{aligned}
\rho^{\mathrm{MF}}(\omega) & =\int_{-\infty}^{\infty} \frac{d k}{2 \pi} \sum_{\alpha} \delta\left(\omega-\alpha E_{k}\right) \\
& =\frac{1}{\pi} \int_{0}^{\infty} d k \delta\left(|\omega|-\sqrt{v_{F}^{2} k^{2}+\Delta_{0}^{2}}\right) \\
& =\rho_{0} \frac{|\omega|}{\sqrt{\omega^{2}-\Delta_{0}^{2}}} \theta\left(\omega^{2}-\Delta_{0}^{2}\right)
\end{aligned}
$$

where the DOS of free fermions with a linearized energy dispersion is given by [see also Eq. (1.47)]

$$
\rho_{0}=\frac{1}{\pi v_{F}} .
$$

A plot of the DOS in the mean-field approximation is shown in Fig. 1.6 Due to the 


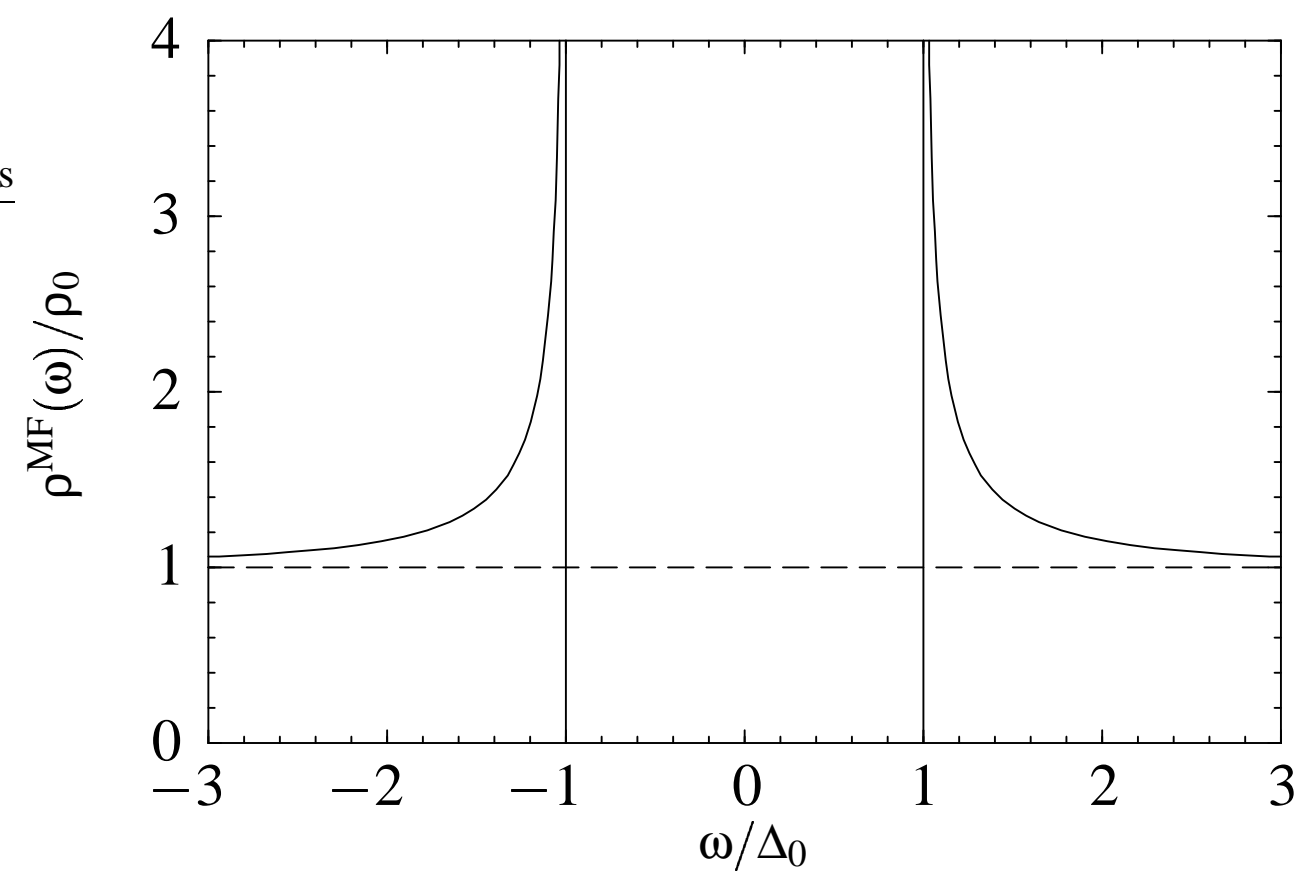

Figure 1.6: Plot of the density of states $\rho^{\mathrm{MF}}(\omega)$ in the mean-field approximation. The DOS vanishes for $|\omega|<\Delta_{0}$ and shows two singularities at $\omega= \pm \Delta_{0}$. For a comparison, the dashed line gives the (constant) DOS of free fermions, $\rho(\omega)=\rho_{0}$.

gap in the electron dispersion, the DOS vanishes for $|\omega|<\Delta_{0}$. The vanishing slope of the energy dispersion $E_{k}$ near the band edges manifests itself in the singularities in the DOS at $\omega= \pm \Delta_{0}$.

\subsubsection{Condensation energy}

The ground state energy can be obtained by filling up all states up to the Fermi level and adding to the corresponding energy the lattice energy $s \rho_{0} L \Delta_{0}^{2} / 2 \lambda$. For a proper normalization we have to subtract from this energy the corresponding energy of free fermions. Since at $T=0$ the free energy coincides with the energy itself, it is not surprising that the above ground state energy is precisely given by Eq. (1.85) leading to the condensation energy [see Eq. (1.88)]

$$
E_{\text {cond }}=-F_{T=0}\left(\Delta_{0}\right)=\frac{s \rho_{0} L}{2} \frac{\Delta_{0}^{2}(0)}{2}
$$

where $\Delta_{0}(0)$ can be expressed by the BCS relation (1.90) in terms of the mean-field transition temperature $T_{c}^{\mathrm{MF}}$. The condensation energy is positive because the Peierls instability leads to a lowering of the occupied energy states near the Fermi level. This energy gain prevails over the energy loss due to the static lattice distortion. 


\subsubsection{Charge-density wave}

The static lattice distortion encountered above is accompanied by a charge-density wave. This can be seen by considering the ground state for which all energy levels below the Fermi energy are occupied, implying the following expectation values:

$$
\left\langle a_{\alpha, k} a_{\alpha^{\prime}, k^{\prime}}\right\rangle=0, \quad\left\langle a_{\alpha, k} a_{\alpha^{\prime}, k^{\prime}}^{\dagger}\right\rangle=\delta_{\alpha, \alpha^{\prime}} \delta_{k, k^{\prime}} \theta(-\alpha k) .
$$

The density operator

$$
\hat{\rho}(x)=\psi^{\dagger}(x) \psi(x)
$$

can be expressed in terms of the field operator

$$
\psi(x)=\frac{1}{\sqrt{L}} \sum_{k} e^{i k x} c_{k}=\frac{1}{\sqrt{L}} \sum_{\alpha, k} e^{i\left(\alpha k_{F}+k\right) x} c_{\alpha, k} .
$$

Recalling that $c_{\alpha, k}=\sum_{\alpha^{\prime}} U_{\alpha, \alpha^{\prime}, k} a_{\alpha^{\prime}, k}$, it follows

$$
\hat{\rho}(x)=\frac{1}{L} \sum_{\alpha, \alpha^{\prime \prime}, k \alpha^{\prime}, \alpha^{\prime \prime \prime}, k^{\prime}} U_{\alpha \alpha^{\prime \prime}, k}^{*} U_{\alpha^{\prime} \alpha^{\prime \prime \prime}, k^{\prime}} e^{-i\left(\alpha k_{F}+k\right) x} e^{i\left(\alpha^{\prime} k_{F}+k^{\prime}\right) x} a_{\alpha^{\prime \prime}, k}^{\dagger} a_{\alpha^{\prime \prime \prime}, k^{\prime}}
$$

Taking the ground-state expectation value and making use of Eqs. (1.97) and (1.105), we get

$$
\begin{aligned}
\langle\hat{\rho}(x)\rangle= & \frac{1}{L} \sum_{\alpha, \alpha^{\prime} \alpha^{\prime \prime} k<0} \sum_{\alpha \alpha^{\prime \prime}, k} U_{\alpha^{\prime} \alpha^{\prime \prime}, k} e^{-i\left(\alpha-\alpha^{\prime}\right) k_{F} x} \\
=\frac{1}{L} \sum_{k>0}\left[1+\left(U_{+-, k}^{*} U_{--, k}+U_{++,-k}^{*} U_{-+,-k}\right) e^{-2 i k_{F} x}\right. & \\
& \left.\quad+\left(U_{--, k}^{*} U_{+-, k}+U_{-+,-k}^{*} U_{++,-k}\right) e^{2 i k_{F} x}\right] .
\end{aligned}
$$

Since $U_{+-, k}^{*}=U_{-+,-k}$, we end up with

$$
\langle\hat{\rho}(x)\rangle=\tilde{\rho}_{0}-\tilde{\rho}_{1} \cos \left(2 k_{F} x+\vartheta\right),
$$

where $\tilde{\rho}_{0}=\pi / k_{0}$ is the charge-density for $\Delta_{0}=0$ and

$$
\tilde{\rho}_{1}=\frac{4}{L} \sum_{0<k<k_{0}}\left(1-\frac{v_{F}^{2} k^{2}}{E_{k}^{2}}\right)^{1 / 2} \sim \frac{\Delta_{0}}{\pi v_{F}^{2} \lambda} .
$$

Below the Peierls transition the system exhibits a charge-density wave with wavevector $2 k_{F}$ and amplitude proportional to the absolute value of the order parameter $|\langle\Delta\rangle|=\Delta_{0}$. 


\subsection{Breakdown of the mean-field picture}

The above mean-field picture was capable of explaining the experimentally observed Peierls transition, including the static lattice distortion, the charge-density wave and the occurrence of a single-particle gap. However, the underlying microscopic machinery contains far more subtleties than one might suspect at first glance. A major point is the importance of fluctuations of the order-parameter field in low-dimensional systems. A theorem due to Mermin and Wagner states that these fluctuations lead to the absence of long-range order, even at very low temperatures [64]. This precludes a spontaneously broken continuous symmetry 7 But how can one explain the experimentally observed charge-density wave which breaks a continuous translational symmetry in strongly anisotropic materials like blue bronze [37]? The answer is simply this: These materials are quasi one-dimensional, but not strictly one-dimensional. As we will see below, in a strictly one-dimensional material, the correlation length $\xi(T)$ increases with decreasing temperature, but for any finite temperature cannot approach infinity. At very low temperatures, however, even very weak interchain-coupling can lead to the onset of three-dimensional order such that the system can undergo a Peierls transition. Of course, the transition temperature is not the previously considered mean-field transition temperature $T_{c}^{\mathrm{MF}}$. Lee, Rice and Anderson [57] pointed out that one should expect $T_{c}^{3 \mathrm{D}} \approx \frac{1}{4} T_{c}^{\mathrm{MF}}$. For a derivation of an adequate three-dimensional microscopic theory see McKenzie [61] and references given therein. Here, we will only consider the strictly one-dimensional case or take the correlation functions of the order parameter field as phenomenological given quantities.

\subsubsection{Correlation functions of the order parameter field}

We will now consider fluctuations of the order parameter field $\Delta(x)$ and calculate the correlation functions of $\Delta(x)$ which describe the phonon statistics.

\section{Harmonic approximation}

At temperatures far above the mean-field critical temperature $T_{c}^{\mathrm{MF}}$, the coefficient $a(T)$ becomes large enough such that anharmonic corrections to the free energy functional may be neglected. Truncating the free energy functional (1.70) at the second order, we are left with

$$
F_{G}\left\{\Delta, \Delta^{*}\right\}=\frac{s \rho_{0} L}{2} \sum_{q}\left[a(T)+c(T) q^{2}\right] \Delta_{q}^{*} \Delta_{q} .
$$

This functional implies the correlation functions

$$
\begin{aligned}
\left\langle\Delta_{q}\right\rangle & =0, \\
\left\langle\Delta_{q} \Delta_{q^{\prime}}^{*}\right\rangle & =\frac{2 k_{B} T}{s \rho_{0} L} \frac{\delta_{q, q^{\prime}}}{a(T)+c(T) q^{2}} .
\end{aligned}
$$

\footnotetext{
${ }^{7}$ It should be noted that the lower critical dimension for this theorem to be true is 2 .
} 
While the correlation functions $\left\langle\Delta_{q} \Delta_{q^{\prime}}\right\rangle$ vanish for complex $\Delta$ they are equal to $\left\langle\Delta_{q} \Delta_{-q^{\prime}}^{*}\right\rangle$ for real $\Delta$. The correlation functions in real space are given by the Fourier transformations of Eqs. (1.114) and (1.115):

$$
\begin{aligned}
\langle\Delta(x)\rangle & =0 \\
\left\langle\Delta(x) \Delta^{*}\left(x^{\prime}\right)\right\rangle & =\Delta_{s}^{2}(T) e^{-\left|x-x^{\prime}\right| / \xi(T)}
\end{aligned}
$$

where

$$
\begin{aligned}
\Delta_{s}^{2}(T) & =\frac{k_{B} T}{s \rho_{0} \sqrt{a(T) c(T)}}, \\
\xi^{-1}(T) & =\left(\frac{a(T)}{c(T)}\right)^{1 / 2} .
\end{aligned}
$$

In the harmonic approximation, higher correlation functions are simply given by Wick's theorem.

\section{Taking into account anharmonic corrections}

As the temperature is lowered and approaches the mean-field critical temperature $T_{c}^{\mathrm{MF}}$, fluctuation effects become important and the mean-field picture breaks down. However, as shown by Scalapino, Sears and Ferrel [79] using the transfer matrix technique, the first two moments of a one-dimensional Ginzburg-Landau theory are still approximately given by Eqs. (1.116) and (1.117). For temperatures well below $T_{c}^{\mathrm{MF}}$, one finds for real $\Delta(x)$ an exponential increase of the correlation length with decreasing temperature, while for complex $\Delta(x)$ the correlation length increases as the inverse temperature. This last result can be understood as follows:

\section{Phase fluctuations only}

For small temperatures $T \ll T_{c}^{\mathrm{MF}}$ the generalized Ginzburg-Landau functional is dominated by its minima. Amplitude fluctuations get frozen out and only phase fluctuations survive such that $\Delta(x) \approx \Delta_{s} e^{i \vartheta(x)}$. For complex $\Delta(x)$, the phase $\vartheta(x)$ is a continuous function of $x$. Ignoring, as before, quartic terms in the gradient expansion of the free energy, the free energy is given up to an irrelevant constant by

$$
\begin{aligned}
F^{\text {(phase })\{V\}} & =F^{\text {(phase) }}\left\{\partial_{x} \vartheta / 2\right\} \\
& =\frac{1}{2} s \rho_{0} \frac{2 \pi}{\beta} \sum_{\tilde{\omega}_{n}>0} \frac{\Delta_{s}^{2}}{\left(\tilde{\omega}_{n}^{2}+\Delta_{s}^{2}\right)^{\frac{3}{2}}} \int_{0}^{L} d x\left(\partial_{x} \vartheta(x) / 2\right)^{2} \\
& =\frac{1}{2} s \rho_{s}(T) \int_{0}^{L} d x V^{2}(x),
\end{aligned}
$$

where

$$
V(x)=\partial_{x} \vartheta(x) / 2
$$




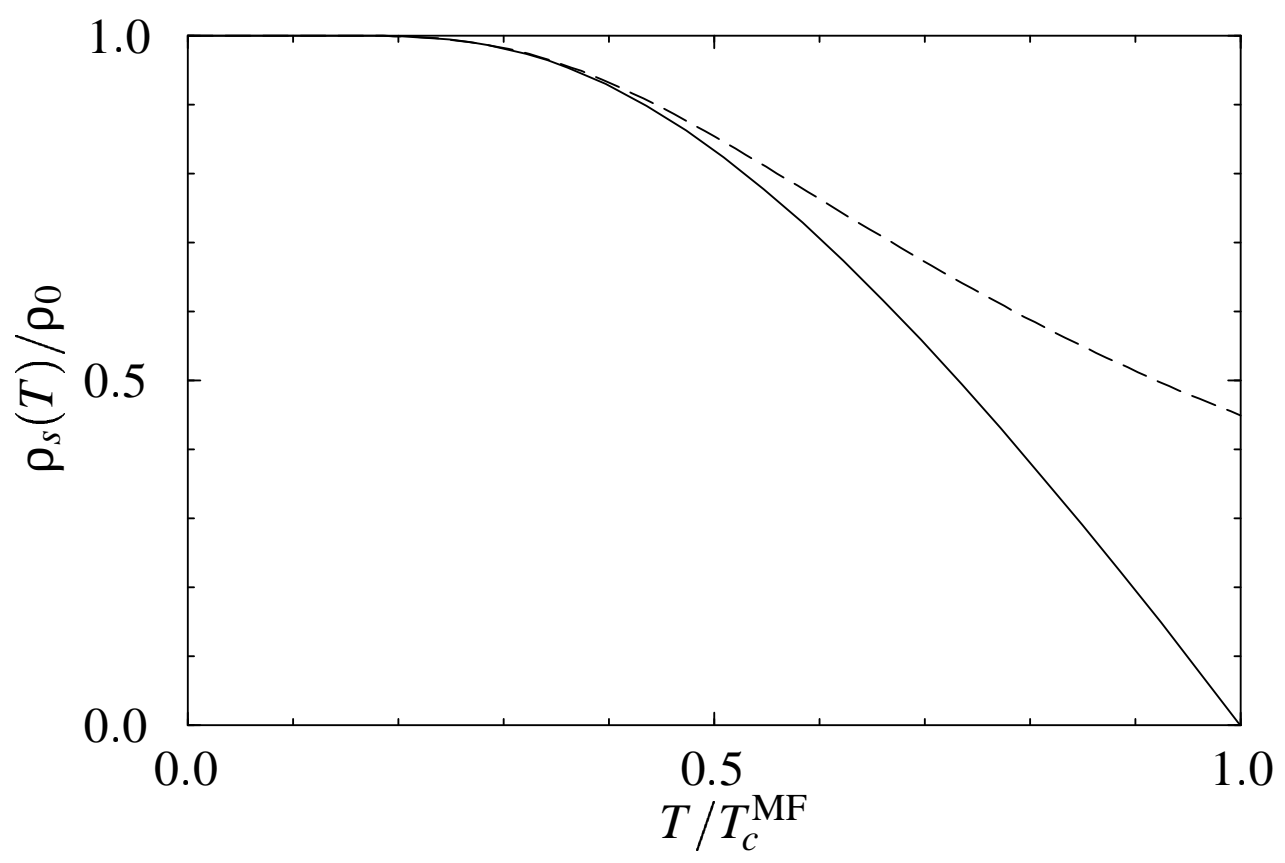

Figure 1.7: Plot of the superfluid density as a function of temperature for $\Delta_{S}=$ $1.76 k_{B} T_{c}^{\mathrm{MF}}$ (dashed line) and $\Delta_{S}(T)$ given by the BCS relation (1.83) (solid line).

can be interpreted (up to a constant $1 / \mathrm{m}^{*}$ ) as the superfluid velocity and

$$
\rho_{s}(T)=\rho_{0} \frac{2 \pi}{\beta} \sum_{\tilde{\omega}_{n}>0} \frac{\Delta_{s}^{2}}{\left(\tilde{\omega}_{n}^{2}+\left|\Delta_{s}\right|^{2}\right)^{\frac{3}{2}}}
$$

is the superfluid density. The free energy is identical to the kinetic energy of a superflow. A two-dimensional analogue of Eq. (1.120) has been used by Emery and Kivelson [25, 26] in their theory describing superconductors with a small phase-stiffness. For $T=0$, the sum in Eq. (1.122) turns into an integral which can be done analytically and gives

$$
\rho_{s}(0)=\rho_{0},
$$

i.e. at $T=0$ is the superfluid density equal to the density of states. Plots of $\rho_{s}(T)$ for $\Delta_{S}(T)$ given by the BCS relation (1.83) and for $\Delta_{S}=1.76 k_{B} T_{c}^{\mathrm{MF}}$ are shown in Fig. 1.7 For small temperatures, $\rho_{s}(T)$ may be approximated by

$$
\rho_{s}(T) \approx \rho_{s}(0), \quad T \lesssim T_{c}^{\mathrm{MF}} / 4 .
$$

Since $F^{\text {(phase) }}$ is only quadratic in $V(x)$, correlation functions of $\Delta(x)$ can easily be calculated: For the first two moments we find

$$
\begin{aligned}
\langle\Delta(x)\rangle & =0, \\
\left\langle\Delta(x) \Delta\left(x^{\prime}\right)\right\rangle & =0,
\end{aligned}
$$


and

$$
\begin{aligned}
\left\langle\Delta(x) \Delta^{*}\left(x^{\prime}\right)\right\rangle & =\Delta_{s}^{2}\left\langle\exp \left(i\left[\vartheta(x)-\vartheta\left(x^{\prime}\right)\right]\right)\right\rangle \\
& =\Delta_{s}^{2}\left\langle\exp \left(2 i \int_{x^{\prime}}^{x} d x^{\prime \prime} V\left(x^{\prime \prime}\right)\right)\right\rangle \\
& =\Delta_{s}^{2} \frac{\mathcal{D}\{V\} \exp \left(2 i \int_{x^{\prime}}^{x} d x^{\prime \prime} V\left(x^{\prime \prime}\right)-s \rho_{s}(T) / 2 k_{B} T \int_{0}^{L} d x^{\prime \prime} V^{2}\left(x^{\prime \prime}\right)\right)}{\mathcal{D}\{V\} \exp \left(-s \rho_{s}(T) / 2 k_{B} T \int_{0}^{L} d x^{\prime \prime} V^{2}\left(x^{\prime \prime}\right)\right)} \\
& =\Delta_{s}^{2} \exp \left(-2 k_{B} T / s \rho_{s}(T)\left|x-x^{\prime}\right|\right) \\
& \equiv \Delta_{s}^{2} \exp \left(-\left|x-x^{\prime}\right| / \xi(T)\right)
\end{aligned}
$$

where

$$
\xi(T)=\frac{s \rho_{s}(T)}{2 k_{B} T}
$$

is the temperature-dependent correlation length. For very small temperatures, $\rho_{s}(T) \approx$ $\rho_{s}(0)=1 / \pi$ [see Eq. (1.124)], such that in this strictly one-dimensional theory we find $\xi(T)=s / 2 \pi k_{B} T \propto 1 / T$ which for fermions with spin $1 / 2$ agrees with Grüner's [37] result $\xi(T)=1 / \pi k_{B} T$.

\subsection{The Hamiltonian of the fluctuating gap model}

Having discussed the statistics of the phonon field, let us now set up the Hamiltonian of the fluctuating gap model (FGM). For a particular realization of the (static) disorder, the electronic part of the Hamiltonian reads

$$
\mathcal{H}=\sum_{k, k^{\prime}}\left(c_{+, k}^{\dagger}, c_{-, k}^{\dagger}\right) H_{k, k^{\prime}}\left(\begin{array}{c}
c_{+, k^{\prime}} \\
c_{-, k^{\prime}}
\end{array}\right)
$$

where

$$
H_{k, k^{\prime}}=\left(\begin{array}{cc}
v_{F} k \delta_{k, k^{\prime}}+V_{k-k^{\prime}} & \Delta_{k-k^{\prime}} \\
\Delta_{-\left(k-k^{\prime}\right)}^{*} & -v_{F} k \delta_{k, k^{\prime}}+V_{k-k^{\prime}}
\end{array}\right) .
$$

A Fourier transformation leads to

$$
\mathcal{H}=\int_{0}^{L} d x\left(\psi_{+}^{\dagger}(x), \psi_{-}^{\dagger}(x)\right) \hat{H}\left(x,-i \partial_{x}\right)\left(\begin{array}{c}
\psi_{+}(x) \\
\psi_{-}(x)
\end{array}\right),
$$

with

$$
\hat{H}\left(x,-i \partial_{x}\right)=\left(\begin{array}{cc}
-i v_{F} \partial_{x}+V(x) & \Delta(x) \\
\Delta^{*}(x) & i v_{F} \partial_{x}+V(x)
\end{array}\right) .
$$

This is the Hamiltonian of the FGM. Recall that we have linearized the energy dispersion such that the FGM can only describe the low-energy physics of Peierls chains in 
the weak-coupling regime. As a further approximation we have considered the phonon field to be static. It will now be the aim to calculate disorder-averaged quantities for the model described by this Hamiltonian. As we will discuss in Chapter 4 instead of averaging over the disorder, it is also possible to consider a typical realization of the disorder potential.

\subsubsection{The fluctuating gap model in other physical contexts}

In this chapter, the fluctuating gap model (FGM) emerged as an effective low-energy model to describe quasi one-dimensional materials which undergo a Peierls transition. Our strictly one-dimensional theory applies only to temperatures above the Peierls transition before three-dimensional fluctuations become important and eventually lead to a phase transition. However, a more sophisticated theory could provide the statistics of the correlation functions of the order parameter field at arbitrary temperatures of an effectively one-dimensional model.

It turns out that the FGM not only describes the low-energy physics of Peierls chains. As shown in Refs. [62] and [17] the Hamiltonian of disordered spin chains [37, 38, 85, 86] can be mapped by a Jordan-Wigner transformation onto the Hamiltonian of the FGM. In a semiclassical approximation of superconductivity, it is also possible to replace the original three-dimensional problem by a directional average over effectively one-dimensional problems [93] which in the weak coupling limit are described by the FGM. This method has been used in Refs. [10, 52, 53] to derive the gradient expansion of a clean superconductor. A generalization of the FGM towards higher dimensions to describe the phase above the phase-transition in underdoped high- $T_{c}$ superconductors by anti-ferromagnetic short-range order fluctuations was considered in Refs. [80] and [81]. 


\section{Chapter 2}

\section{The Green function and related quantities}

In this chapter, we will introduce different concepts to calculate the Green function and related quantities of the fluctuating gap model. The density of states and the localization length will be of special interest. In particular, we will develop a method which allows to calculate these quantities simultaneously for arbitrary given disorder potentials. In this chapter, we will only calculate the averaged single-particle Green function in the Born approximation. Detailed non-perturbative calculations based on the formalism to be developed in this chapter will be postponed to the next chapters.

As we have seen in the last chapter, the fluctuating gap model (FGM) describes the low-energy physics of one-dimensional fermions subject to static disorder potentials and applies to different physical contexts. The Hamiltonian of the FGM is of the Dirac type and in first quantized form can be written as [see Eq. (1.132)]

$$
\hat{H}\left(x,-i \partial_{x}\right)=-i v_{F} \partial_{x} \sigma_{3}+V(x) \sigma_{0}+\Delta(x) \sigma_{+}+\Delta^{*}(x) \sigma_{-} .
$$

$V(x)$ and $\Delta(x)$ are random potentials describing forward and backward scattering, $v_{F}$ is the Fermi velocity (henceforth we set $v_{F}=1$ ), $\sigma_{i}$ are the usual Pauli matrices, $\sigma_{0}$ is the $2 \times 2$ unit matrix, and $\sigma_{ \pm}=\frac{1}{2}\left(\sigma_{1} \pm i \sigma_{2}\right)$.

\subsection{The Green function}

In the following, we are going to consider the retarded Green function $\mathcal{G}^{R}\left(x, x^{\prime} ; \omega\right)$ of the fluctuating gap model. This quantity is of special interest because it can be related to several quantities which are in principle experimentally accessible. The trace of the imaginary part of the Green function at coinciding space points determines the local density of states (DOS),

$$
\rho(x, \omega)=-\pi^{-1} \operatorname{Im} \operatorname{Tr}\left[\mathcal{G}^{R}(x, x ; \omega)\right] .
$$


Averaging $\rho(x, \omega)$ over all space points gives the DOS $\rho(\omega)$, which is the fundamental quantity that determines the whole thermodynamics of the FGM. It will turn out that the trace of the energy-integrated space averaged Green function at coinciding space points $\Gamma(\omega)$ will be easier to calculate than its non-integrated form. While its imaginary part is proportional to the integrated DOS $\mathcal{N}(\omega)$, the Thouless formula states that $\operatorname{Re} \Gamma(\omega)$ is equal to the inverse localization length $\ell^{-1}(\omega)$. As in the following chapters, we will usually only consider the DOS and the inverse localization length for positive frequencies $\omega$. Due to the particle-hole symmetry, the DOS and the localization length are symmetric with respect to the Fermi energy so that after setting the Fermi energy equal to zero we have $\rho(\omega)=\rho(-\omega)$ and $\ell^{-1}(\omega)=\ell^{-1}(-\omega)$. It therefore suffices to consider the case $\omega>0$.

The retarded $2 \times 2$ matrix Green function $\mathcal{G}^{R}\left(x, x^{\prime} ; \omega\right)$ to the Schrödinger operator $\omega-\hat{H}$ satisfies the differential equation

$$
\left[\omega+i 0^{+}-\hat{H}\left(x,-i \partial_{x}\right)\right] \mathcal{G}^{R}\left(x, x^{\prime} ; \omega\right)=\delta\left(x-x^{\prime}\right) \sigma_{0} .
$$

The positive but infinitesimal imaginary part added to the frequency $\omega$ indicates that we have to impose the correct boundary conditions applying to a retarded Green function.

\subsubsection{Free fermions}

It is easy to calculate the Green function for free fermions. In this case, $V(x)=\Delta(x)=$ 0 , such that the system is translational invariant, and Eq. (2.3) simplifies to

$$
\left[\omega+i 0^{+}+i \sigma_{3} \partial_{x}\right] \mathcal{G}_{0}^{R}\left(x-x^{\prime} ; \omega\right)=\sigma_{0} \delta\left(x-x^{\prime}\right) .
$$

Taking the Fourier transform of this equation from real space to momentum space gives

$$
\left[\omega+i 0^{+}-k \sigma_{3}\right] G_{0}^{R}(k ; \omega)=\sigma_{0} .
$$

$\mathcal{G}_{0}^{R}(k ; \omega) \equiv \int d x e^{-i k x} \mathcal{G}_{0}^{R}(x ; \omega)$ can now be found by a simple matrix inversion. If $\alpha=1$ accounts for right- and $\alpha=-1$ for left-moving fermions, the matrix elements of $\mathcal{G}_{0}^{R}(k ; \omega)$ are given by

$$
\left(G_{0}^{R}\right)_{\alpha \alpha^{\prime}}(k ; \omega)=\frac{\delta_{\alpha, \alpha^{\prime}}}{\omega-\alpha k+i 0^{+}} .
$$

A simple Fourier transformation back to real space now gives the retarded propagator of free fermions in real space,

$$
i\left(\mathcal{G}_{0}^{R}\right)_{\alpha \alpha^{\prime}}(x ; \omega)=\delta_{\alpha, \alpha^{\prime}} \theta(\alpha x) e^{i \alpha \omega x} .
$$

Here, $\theta(x)$ is the Heaviside step function

$$
\theta(x)=\left\{\begin{array}{ll}
0, & x<0 \\
1, & x>0
\end{array} .\right.
$$


For concreteness, let us also define $\theta(0)=\lim _{x \rightarrow 0}[\theta(x)+\theta(-x)] / 2=1 / 2$. While the matrix elements at $x=0$ are sensitive to the definition $\theta(0)=1 / 2$ which amounts to defining

$$
\mathcal{G}^{R}(x=0 ; \omega) \equiv \frac{1}{2} \lim _{x \rightarrow 0^{+}}\left[\mathcal{G}^{R}(+x ; \omega)+\mathcal{G}^{R}(-x ; \omega)\right],
$$

the local DOS $\rho(x, \omega)=-\pi^{-1} \operatorname{Im} \operatorname{Tr}\left[\mathcal{G}_{0}^{R}(0 ; \omega)\right]$ does not depend on this definition because it only involves the harmless quantity $\theta(x)+\theta(-x)=1$. Due to translational symmetry, the total DOS is equal to the space-independent local DOS,

$$
\rho_{0}(\omega)=\pi^{-1} \text {. }
$$

Note that the DOS of free fermions is independent of the frequency because we have linearized the energy dispersion.

A further Fourier transformation of Eq. (2.7) from frequency to time gives

$$
i\left(\mathcal{G}_{0}^{R}\right)_{\alpha \alpha^{\prime}}(x ; t)=\delta_{\alpha, \alpha^{\prime}} \theta(t) \delta(\alpha x-t) .
$$

This free retarded Green function in space and time allows for a simple interpretation: A fermion put into the system at $t^{\prime}=0$ as a right- or left-mover will at time $t>0$ have traveled a distance $|x|=t=v_{F} t$ in the positive or negative direction, respectively. The fermion can not be observed in the system at times $t<0$.

\subsection{Dyson equation and perturbation theory}

One way to handle the disorder is to consider the disorder potential as a perturbation and expand the Green function in powers of this potential. Defining $\mathbf{V}(x) \equiv V(x) \sigma_{0}+$ $\Delta(x) \sigma_{+}+\Delta^{*}(x) \sigma_{-}$, Eq. (2.3) may be written as

$$
\left[i \sigma_{3} \partial_{x}+\omega+i 0^{+}\right] \mathcal{G}^{R}\left(x, x^{\prime} ; \omega\right)=\delta\left(x-x^{\prime}\right) \sigma_{0}+\mathbf{V}(x) \mathcal{G}^{R}\left(x, x^{\prime} ; \omega\right) .
$$

Substituting $x$ by $x_{1}$, multiplying the resulting equation from the left with the free Green function $\mathcal{G}_{0}^{R}\left(x-x_{1} ; \omega\right)$ and then integrating over $x_{1}$ gives the Dyson equation

$$
\mathcal{G}^{R}\left(x, x^{\prime} ; \omega\right)=\mathcal{G}_{0}^{R}\left(x-x^{\prime} ; \omega\right)+\int d x \mathcal{G}_{0}^{R}\left(x-x_{1} ; \omega\right) \mathbf{V}\left(x_{1}\right) \mathcal{G}^{R}\left(x_{1}, x^{\prime} ; \omega\right) .
$$

Iterating this Dyson equation, the exact Green function can be expressed in terms of the free Green function and the disorder potential:

$$
\mathcal{G}^{R}\left(x, x^{\prime} ; \omega\right)=\sum_{n=0}^{\infty} \mathcal{G}_{n}^{R}\left(x, x^{\prime} ; \omega\right),
$$

where $\mathcal{G}_{0}^{R}\left(x, x^{\prime} ; \omega\right)=\mathcal{G}_{0}^{R}\left(x-x^{\prime} ; \omega\right)$ is the free Green function calculated above, and for $n \geq 1$ the functions $\mathcal{G}_{n}^{R}\left(x, x^{\prime} ; \omega\right)$ are given by

$$
\begin{aligned}
& \mathcal{G}_{n}^{R}\left(x, x^{\prime} ; \omega\right)=\int d x_{1} \ldots \int d x_{n} \\
& \quad \mathcal{G}_{0}^{R}\left(x-x_{n} ; \omega\right) \mathbf{V}\left(x_{n}\right) \mathcal{G}_{0}^{R}\left(x_{n}-x_{n-1} ; \omega\right) \ldots \mathbf{V}\left(x_{1}\right) \mathcal{G}_{0}^{R}\left(x_{1}-x^{\prime} ; \omega\right) .
\end{aligned}
$$




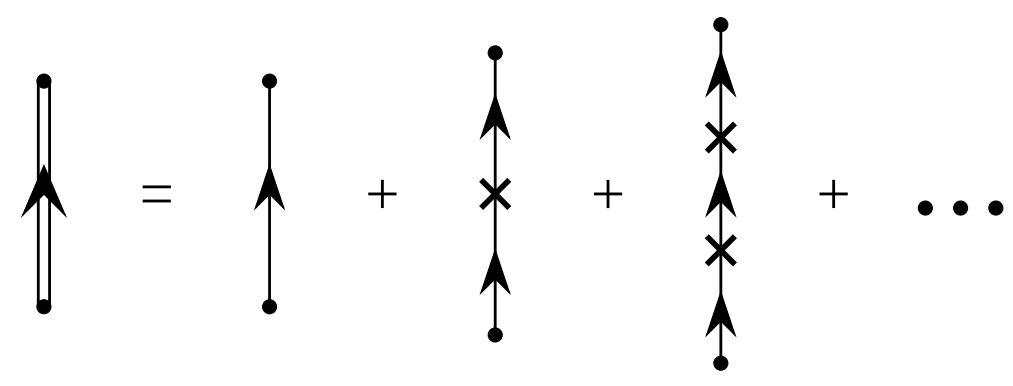

Figure 2.1: Diagrammatic expansion of the matrix Green function. While the single line represents the Green function of free fermions, the double line is a graphical representation of the full Green function $\mathcal{G}^{R}\left(x, x^{\prime} ; \omega\right)$. The crosses denote the disorder potential $\mathbf{V}(x)$.

Recall that the right-hand side of this equation involves the product of $2 \times 2$-matrices. The perturbative expansion of the full Green function can be visualized by using Feynman diagrams (see Fig. 2.1).

The physical interpretation of the perturbation expansion is simple: The perturbative expansion takes into account all possibilities of a particle moving through the sample getting scattered at the various impurity potentials. While $\Delta(x)$ changes the direction in which the particle travels and therefore can be interpreted as a backscattering potential, $V(x)$ does not change the direction of the particle, so that it only leads to forward scattering.

\subsubsection{Boundary conditions of the retarded Green function}

Below, we will consider a non-perturbative approach to calculate the Green function of the FGM. The above perturbative expansion can be used to obtain the correct boundary conditions of the full retarded Green function: Let us consider $\left(G_{n}^{R}\right)_{\alpha \alpha^{\prime}}\left(x, x^{\prime} ; \omega\right)$. According to Eq. (2.15), its expansion in a product of free Green functions and the potentials starts with $\left(\mathcal{G}_{0}^{R}\right)_{\alpha \alpha}\left(x-x_{n} ; \omega\right)$ and ends with $\left(\mathcal{G}_{0}^{R}\right)_{\alpha^{\prime} \alpha^{\prime}}\left(x_{1}-x^{\prime} ; \omega\right)$. These terms are proportional to $\theta\left(\alpha\left(x-x_{n}\right)\right)$ and $\theta\left(\alpha^{\prime}\left(x_{1}-x^{\prime}\right)\right)$, respectively, so that $\left(\mathcal{G}_{n}^{R}\right)_{\alpha \alpha^{\prime}}\left(x, x^{\prime} ; \omega\right)$ has to vanish as $\alpha x \rightarrow-\infty$ or $\alpha^{\prime} x^{\prime} \rightarrow \infty$. Since this reasoning applies to all orders in perturbation theory, it also applies to the full Green function. If we demand the potentials to vanish outside the interval $[-\Lambda, L+\Lambda]$, the boundary condition can also be written as

$$
\left(\mathcal{G}^{R}\right)_{\alpha \alpha^{\prime}}\left(-\alpha \Lambda, x^{\prime} ; \omega\right)=0, \quad\left(\mathcal{G}^{R}\right)_{\alpha \alpha^{\prime}}\left(x, \alpha^{\prime}(L+\Lambda) ; \omega\right)=0
$$




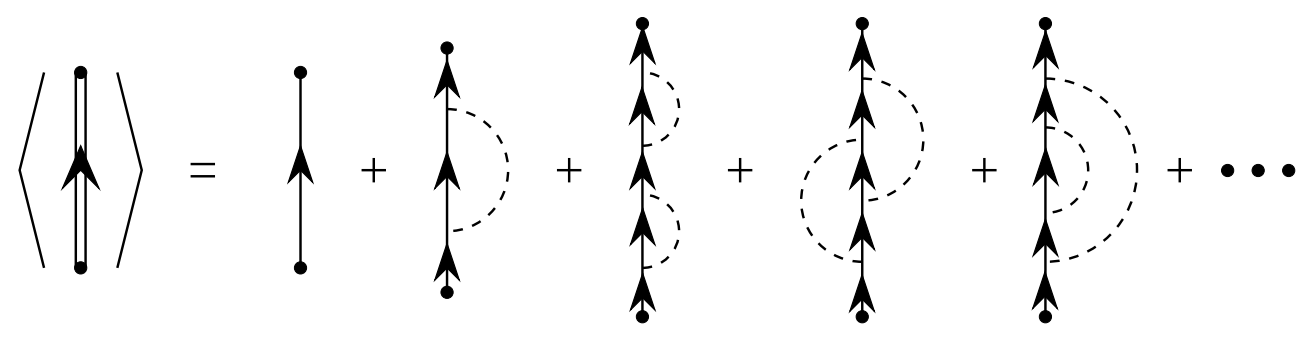

Figure 2.2: Diagrammatic representation of the averaged matrix Green function. The single line represents the Green function of free fermions and in this case the (averaged) double line is a graphical representation of the full (averaged) Green function $\left\langle\mathcal{G}^{R}\left(x, x^{\prime} ; \omega\right)\right\rangle$. The dashed line denotes the disorder average $\left\langle\mathbf{V}(x) \mathbf{V}\left(x^{\prime}\right)\right\rangle$.

\subsection{Second order Born approximation}

Let us now consider the disorder-averaged Green function. As discussed in Chapter 1 above the Peierls transition the first two moments of the order parameter field $\Delta(x)$ are given by

$$
\langle\Delta(x)\rangle=0, \quad\left\langle\Delta(x) \Delta^{*}\left(x^{\prime}\right)\right\rangle=\Delta_{s}^{2} e^{-\left|x-x^{\prime}\right| / \xi} .
$$

Following Lee, Rice and Anderson [57], we ignore the forward scattering disorder, i.e. set $V(x)=0$. For a perturbative approach we assume Gaussian statistics for the higher moments of the order parameter field such that these moments can be separated according to Wick's theorem. A diagrammatic representation of the averaged Green function is shown in Fig. 2.2 An infinite number of diagrams can be summed up by introducing irreducible diagrams which by definition cannot be separated into two disconnected diagrams by cutting a single propagator. The corresponding amputated diagram is obtained by eliminating all outer propagators. The sum of all amputated irreducible diagrams is known as the self-energy and is diagrammatically presented in Fig. 2.3. In terms of the self-energy, the averaged Green function reads in momentum

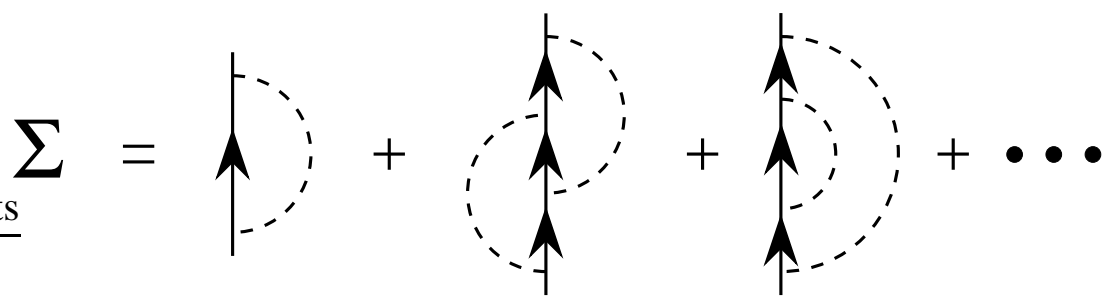

Figure 2.3: Diagrammatic representation of the (irreducible) self-energy. As in the above Figure 2.2, the single line represents the Green function of free fermions and the dashed line denotes the disorder average $\left\langle\mathbf{V}(x) \mathbf{V}\left(x^{\prime}\right)\right\rangle$. 
space

$$
\left\langle\mathcal{G}^{R}(k ; \omega)\right\rangle=\left[\left(\mathcal{G}_{0}^{R}(k ; \omega)\right)^{-1}-\Sigma(k ; \omega)\right]^{-1} .
$$

The simplest approximation to take into account fluctuation effects of the order parameter is to consider only the first diagram in Fig. 2.3 This approximation is known as the second order Born approximation and is essentially the approximation made by Lee, Rice and Anderson in their seminal paper [57] in which fluctuations of the order parameter of the FGM were taken into account for the first time. A special nonGaussian probability distribution of $\Delta(x)$ involving only phase fluctuations for which the second order Born approximation turns out to be exact is presented in Ref. [13].

\subsubsection{The self-energy}

Since $\langle\mathbf{V}(x)\rangle=0$, the self-energy in the second order Born approximation is given by

$$
\Sigma_{B}\left(x-x^{\prime} ; \omega\right)=\left\langle\mathbf{V}(x) \mathcal{G}_{0}^{R}\left(x-x^{\prime} ; \omega\right) \mathbf{V}\left(x^{\prime}\right)\right\rangle .
$$

Placing Eq. (2.7) into this equation, we get

$$
\begin{aligned}
\left(\Sigma_{B}\right)_{\alpha \alpha^{\prime}}\left(x-x^{\prime} ; \omega\right) & =\delta_{\alpha, \alpha^{\prime}} \Delta_{s}^{2} e^{-\left|x-x^{\prime}\right| / \xi}\left(G_{0}^{R}\right)_{\bar{\alpha}, \bar{\alpha}}\left(x-x^{\prime} ; \omega\right) . \\
& =-i \delta_{\alpha, \alpha^{\prime}} \Delta_{s}^{2} \theta\left(-\alpha\left(x-x^{\prime}\right)\right) e^{-i \alpha[\omega+i / \xi]\left(x-x^{\prime}\right)}
\end{aligned}
$$

As one should expect, the process of averaging restored translational invariance. Taking the Fourier transform of Eq. 2.20), we arrive at

$$
\begin{aligned}
\left(\Sigma_{B}\right)_{\alpha \alpha^{\prime}}(k ; \omega) & =\int d x e^{-i k x}\left(\Sigma_{B}\right)_{\alpha \alpha^{\prime}}(x ; \omega) \\
& =\delta_{\alpha, \alpha^{\prime}} \frac{\Delta_{s}^{2}}{\omega+\alpha k+i / \xi}
\end{aligned}
$$

Within second order Born approximation, we therefore find for the one-particle Green function

$$
\left(G_{B}^{R}\right)_{\alpha, \alpha^{\prime}}(k ; \omega)=\frac{\delta_{\alpha, \alpha^{\prime}}}{\omega-\alpha k-\frac{\Delta_{s}^{2}}{\omega+\alpha k+i / \xi}} .
$$

This result was first obtained by Lee, Rice and Anderson [57].

\subsubsection{The density of states and the inverse localization length}

Integrating Eq. (2.22) over $k$ and taking the trace, we obtain

$$
\operatorname{Tr} \mathcal{G}_{B}^{R}(x, x ; \omega)=-i \frac{\omega+i / 2 \xi}{\sqrt{(\omega+i / 2 \xi)^{2}-\Delta_{s}^{2}}},
$$


where $\sqrt{z}$ is defined as the principal part of the square root with the cut chosen along the negative real axis.

The imaginary part of Eq. (2.23) gives the (averaged) DOS,

$$
\rho_{B}(\omega)=\rho_{0} \operatorname{Re} \frac{\omega+i / 2 \xi}{\sqrt{(\omega+i / 2 \xi)^{2}-\Delta_{s}^{2}}} .
$$

As we will show in Section 2.8 the real part of the Green function is equal to the derivative of the inverse localization length $\ell^{-1}(\omega)$. Hence,

$$
\partial_{\omega} \ell_{B}^{-1}(\omega)=\operatorname{Im} \frac{\omega+i / 2 \xi}{\sqrt{(\omega+i / 2 \xi)^{2}-\Delta_{s}^{2}}} .
$$

Integrating this equation with respect to $\omega$ and choosing the integration constant at infinity equal to zero, we obtain

$$
\ell_{B}^{-1}(\omega)=\operatorname{Im} \sqrt{(\omega+i / 2 \xi)^{2}-\Delta_{s}^{2}}-1 / 2 \xi .
$$

A plot of both $\rho_{B}(\omega)$ and $\ell_{B}^{-1}(\omega)$ is shown for different values of the dimensionless parameter $\Delta_{s} \xi$ in Fig. 2.4 With increasing correlation length $\xi$ the DOS gets more and more suppressed for $\omega \lesssim \Delta_{s}$. However, instead of a real gap the fluctuations can only create a pseudogap. At $\omega=0$, the DOS is given by

$$
\rho_{B}(0)=\rho_{0} \frac{1}{\sqrt{1+\left(2 \Delta_{s} \xi\right)^{2}}},
$$

such that for $\Delta_{s} \xi \gg 1$ the DOS vanishes as

$$
\rho_{B}(0) \sim \frac{\rho_{0}}{2 \Delta_{s} \xi} \propto \frac{1}{\Delta_{s} \xi} .
$$

For large frequencies $\omega$, the asymptotic form of the DOS is given by

$$
\rho_{B}(\omega) \sim \rho_{0}\left[1+\frac{\Delta_{s}^{2}}{2 \omega^{2}}\right]
$$

Finally we note that as the correlation length approaches infinity, the DOS assumes the mean-field result given in Eq. (1.102).

The analogous expressions for the inverse localization length are also easily calculated: At zero frequency, the inverse localization length is given by

$$
\ell_{B}^{-1}(0)=\Delta_{s}\left[\sqrt{1+\left(1 / 2 \Delta_{s} \xi\right)^{2}}-1 / 2 \Delta_{s} \xi\right] .
$$

And for large frequencies, $\ell_{B}^{-1}(\omega)$ vanishes asymptotically as

$$
\ell_{B}^{-1}(\omega) \sim \frac{\Delta_{s}^{2}}{4 \omega^{2 \xi}}
$$



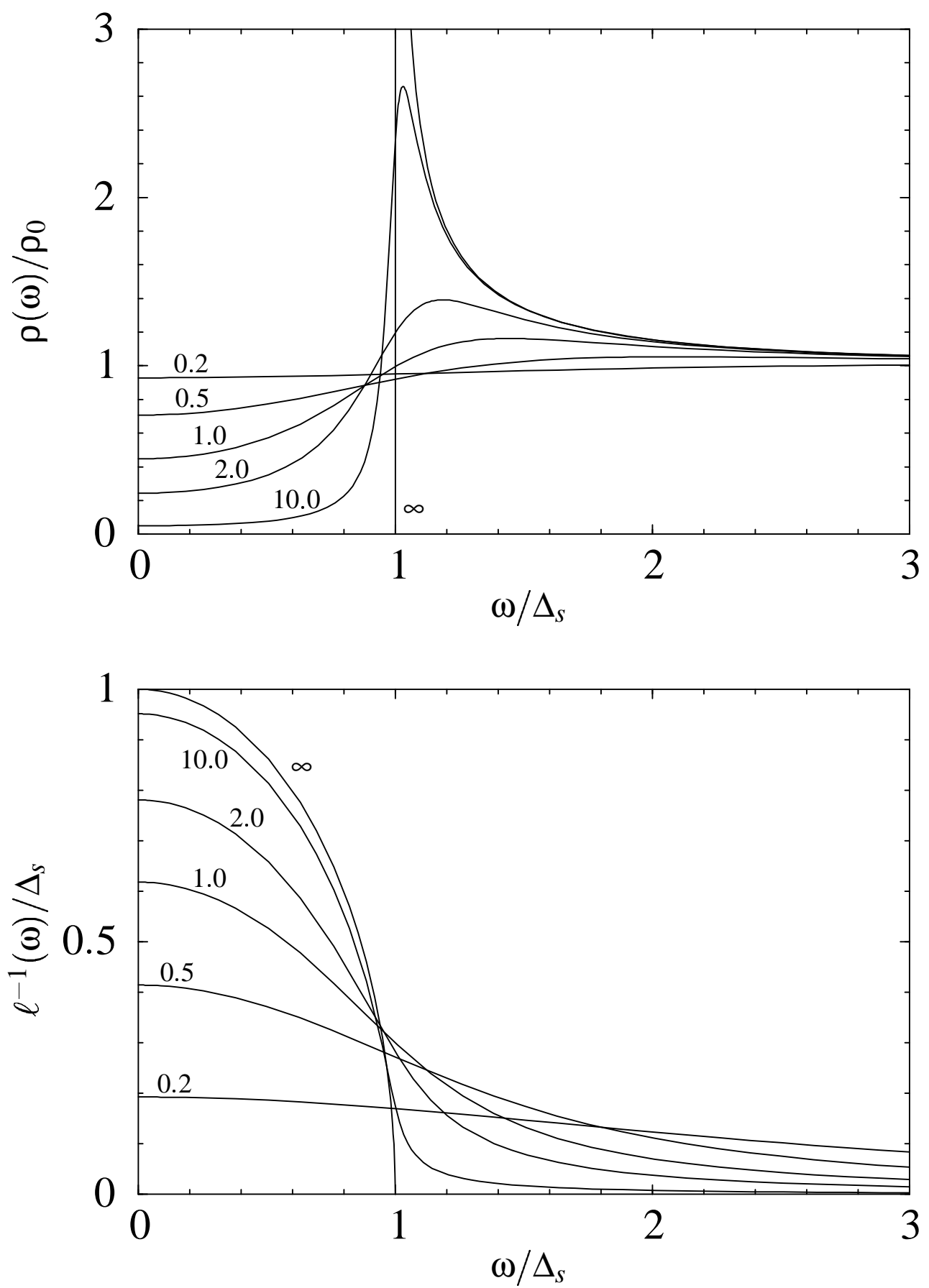

Figure 2.4: Plot of the DOS $\rho_{B}(\omega)$ and the inverse localization length $l_{B}^{-1}(\omega)$ calculated for $\Delta_{s} \xi=0.2,0.5,1.0,2.0,10.0$, and $\infty$ (mean-field result) in the second order Born approximation. 


\subsubsection{The spectral function}

Another interesting quantity related to the single-particle Green function is the spectral function

$$
\rho\left(\alpha k_{F}+k ; \omega\right)=-\frac{1}{\pi} \operatorname{Im}\left(\mathcal{G}^{R}(k ; \omega)\right)_{\alpha, \alpha} .
$$

Experimentally, the spectral function can be measured by angular resolved photoemission spectroscopy (ARPES). It directly follows from Eq. (2.22) that in the Born approximation the spectral function is given by

$$
\rho_{B}\left(\alpha k_{F}+k ; \omega\right)=\rho_{0} \frac{\Delta_{s}^{2 \xi}}{\left(\Delta_{s}^{2}-\left(\omega^{2}-k^{2}\right)\right)^{2} \xi^{2}+(\omega-\alpha k)^{2}} .
$$

Plots of the spectral function $\rho_{B}\left(\alpha k_{F} ; \omega\right)$ and $\rho_{B}\left(\alpha k_{F}+k ; \omega\right)$ with $k=0.5 \Delta_{s}$ as functions of $\omega$ are shown for different values of $\Delta_{S} \xi$ in Figs. 2.5 and 2.6. While for small correlation lengths, i.e. $\Delta_{s} \xi \ll 1$ the spectral function exhibits a maximum near $\omega=\alpha k$, for large correlation lengths we find two maxima near $\omega= \pm \sqrt{\Delta_{s}^{2}+k^{2}}$, the closest one to $\alpha k$ having the larger weight.

\section{Sadovskii's solution and corrections to the second order Born approximation}

An attempt to sum up all diagrams in the perturbative expansion of the averaged Green function was made by Sadovskii in the late seventies [78]. His solution was known as the only available exact solution of the pseudo-gap state (see Ref. [87]) and was therefore also used by other authors [63, 80, 81]. However, only recently Tchernyshyov discovered an unfortunate error in Sadovskii's solution which also turned the work based on it into question [87]. For an analysis of the failure of Sadovskii's solution see the clarifying paper [87]. Instead of trying to sum up all diagrams in the perturbative expansion of the Green function we will now develop a method which will allow for a non-perturbative calculation of the Green function.

\subsection{Non-Abelian Schwinger-ansatz}

We base our non-perturbative approach to calculate the Green function of the FGM on a matrix generalization of the Schwinger-ansatz [84]. To make the differential operator $-i \partial_{x}$ proportional to the unit matrix, we first factor out a Pauli matrix $\sigma_{3}$, so that the retarded Green function

$$
\tilde{\mathcal{G}}^{R}\left(x, x^{\prime} ; \omega\right)=\sigma_{3} \mathcal{G}^{R}\left(x, x^{\prime} ; \omega\right)
$$

satisfies

$$
\left[i \partial_{x}-M\left(x, \omega+i 0^{+}\right)\right] \tilde{\mathcal{G}}^{R}\left(x, x^{\prime} ; \omega\right)=\delta\left(x-x^{\prime}\right) \sigma_{0},
$$

where

$$
M(x, \omega)=\left[V(x)-\omega+\Delta(x) \sigma_{+}+\Delta^{*}(x) \sigma_{-}\right] \sigma_{3}
$$




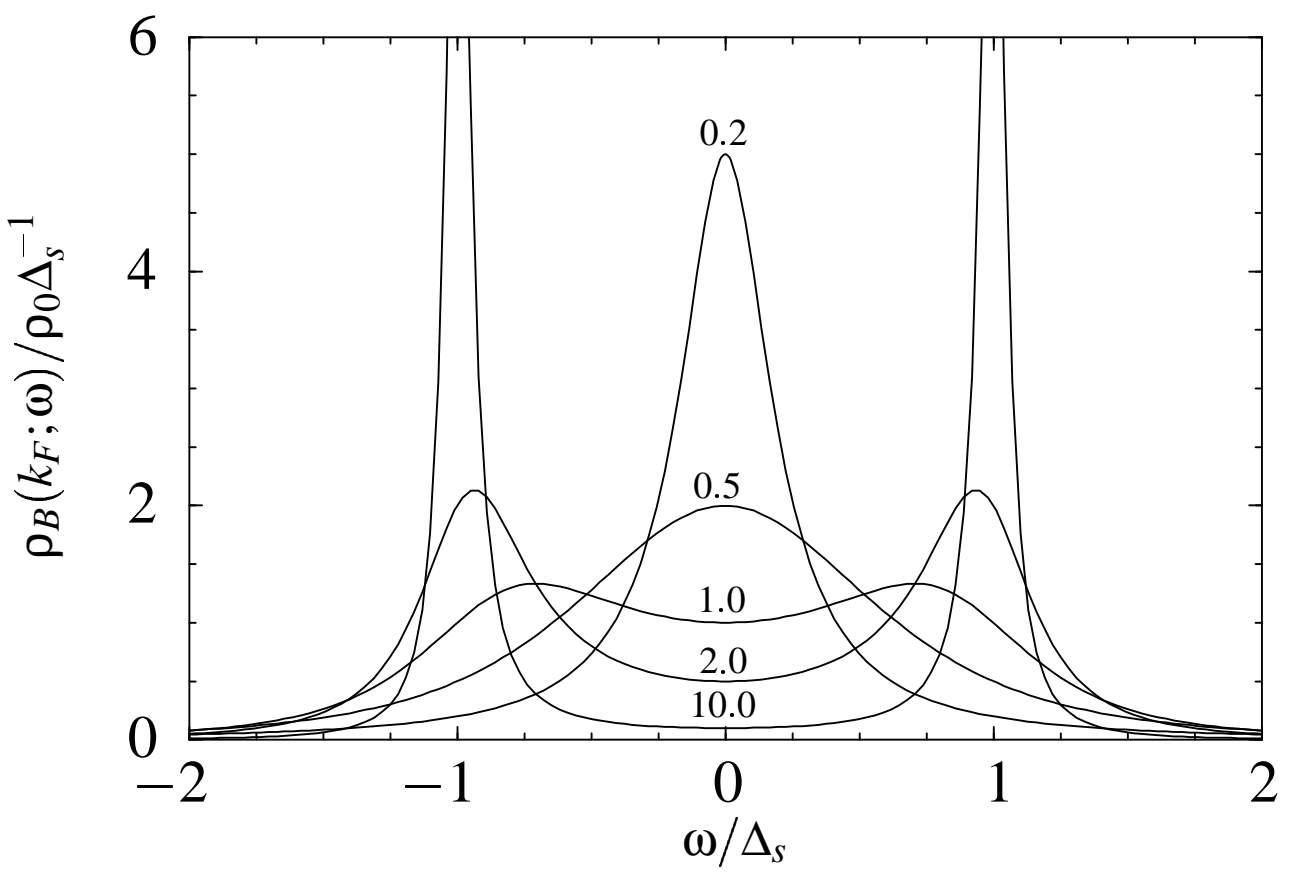

Figure 2.5: Plot of the spectral function $\rho_{B}\left(k_{F} ; \omega\right)$ calculated for $\Delta_{s} \xi=$ $0.2,0.5,1.0,2.0,10.0$ in the second order Born approximation.

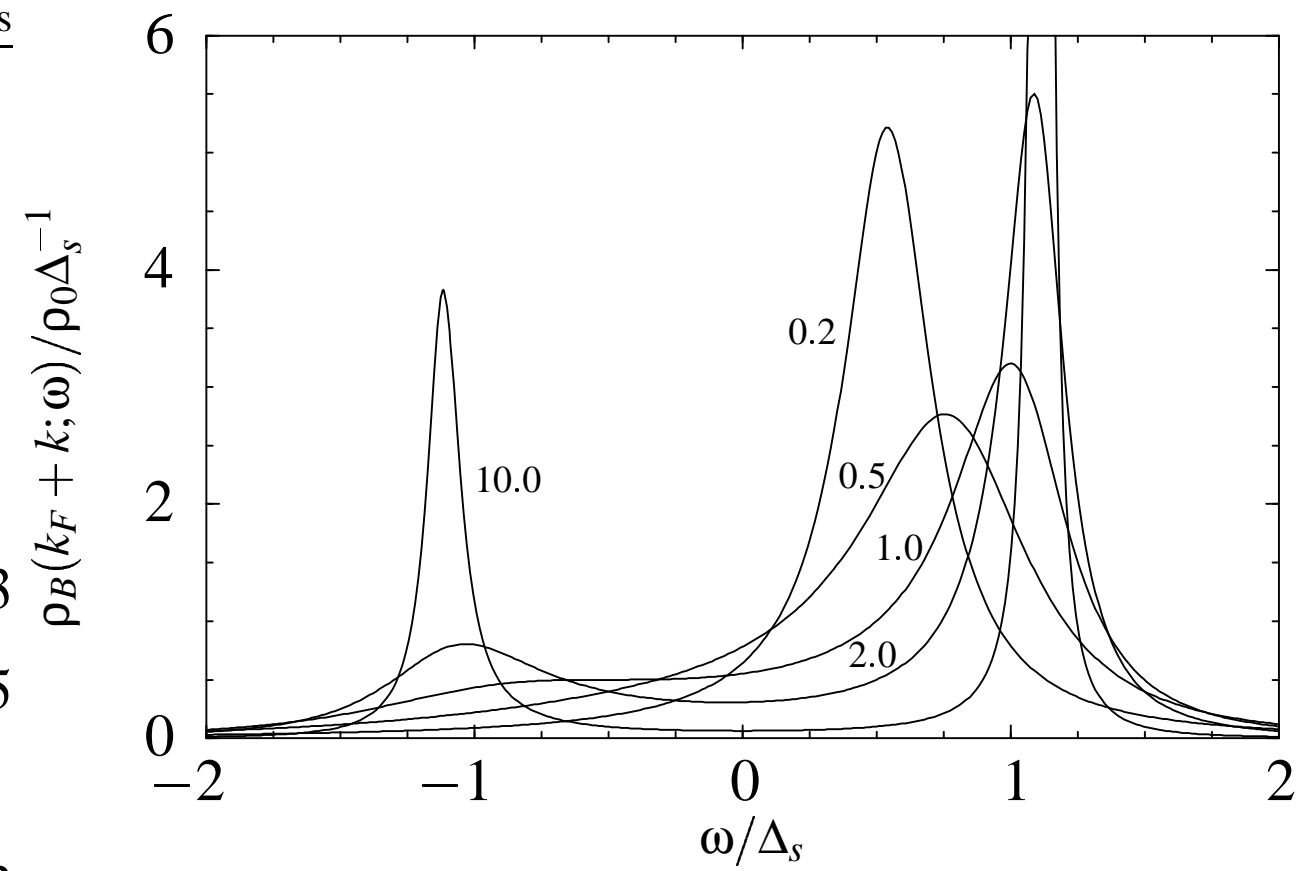

Figure 2.6: Plot of the spectral function $\rho_{B}\left(k_{F}+k ; \omega\right)$ with $k=0.5 \Delta_{s}$ calculated for $\Delta_{s} \xi=0.2,0.5,1.0,2.0,10.0$ in the second order Born approximation. 
is a traceless matrix. We now try to solve Eq. (2.35) by making the ansatz

$$
\tilde{\mathcal{G}}^{R}\left(x, x^{\prime}, \omega\right)=U(x, \omega) \tilde{\mathcal{G}}_{0}^{R}\left(x-x^{\prime}\right) U^{-1}\left(x^{\prime}, \omega\right),
$$

where $U(x, \omega)$ is an invertible $2 \times 2$ matrix and $\tilde{\mathcal{G}}_{0}^{R}(x)$ is the Green function to the operator $i \partial_{x}+i 0^{+} \sigma_{3}$, i.e.

$$
\tilde{\mathcal{G}}_{0}^{R}(x)=-i\left(\begin{array}{cc}
\theta(x) & 0 \\
0 & -\theta(-x)
\end{array}\right) .
$$

The ansatz (2.37) resembles the transformation law for the comparator in nonAbelian Gauge theory (see, for example, the book on quantum field theory by Peskin and Schröder [73]), and since it is also similar to the scalar Schwinger-ansatz [84] which is sometimes used in functional bosonization of interacting fermions [7. 9. 48, 49, 50, 51], we will refer to it as the non-Abelian Schwinger-ansatz [8] 10, 11]. But note that in contrast to earlier formulations of the non-Abelian Schwinger-ansatz [8, 10], we use the zero-frequency free retarded Green function, such that the whole $\omega$ dependence is included in $U(x, \omega)$.

In the following we are going to suppress the parameter $\omega$. The ansatz (2.37) indeed solves Eq. 2.35) if $U(x)$ satisfies

$$
\left[i \partial_{x}-M(x)\right] U(x)=0 .
$$

To establish our formalism, let us first restrict the disorder potentials to the interval $(-\Lambda, L+\Lambda)$. While the potentials are assumed to be constant in the intervals $(-\Lambda, 0)$ and $(L, L+\Lambda)$, they are allowed to fluctuate in between. An example is given in Fig. (2.7). Later we can let $\Lambda \rightarrow \infty$ (or we will set $\Lambda=0$ and let $L \rightarrow \infty$ ). The boundary conditions for the retarded Green function given in Eq. (2.16) now renders into 1

$$
U_{12}(-\Lambda)=U_{21}(L+\Lambda)=0
$$

Two different solutions of Eq. (2.39) are given by

$$
\begin{aligned}
& U_{+}(x)=T \exp \left[-i \int_{-\Lambda}^{x} M(y) d y\right], \\
& U_{-}(x)=T^{-1} \exp \left[i \int_{x}^{L+\Lambda} M(y) d y\right],
\end{aligned}
$$

where $T \exp$ is the path-ordered and $T^{-1} \exp$ is the anti-path-ordered exponential function. Both, $U_{+}(x)$ and $U_{-}(x)$ can be expressed in terms of the $S$-matrix,

$$
S\left(x, x^{\prime}\right)=T \exp \left[-i \int_{x^{\prime}}^{x} M(y) d y\right] .
$$

\footnotetext{
${ }^{1}$ In this work we will identify matrix elements as $U_{i j}$ with $U_{\alpha \alpha^{\prime}}$, where $i, j=1,2$ corresponds to $\alpha, \alpha^{\prime}=+,-$, e.g. $U_{12} \equiv U_{+-}$.
} 


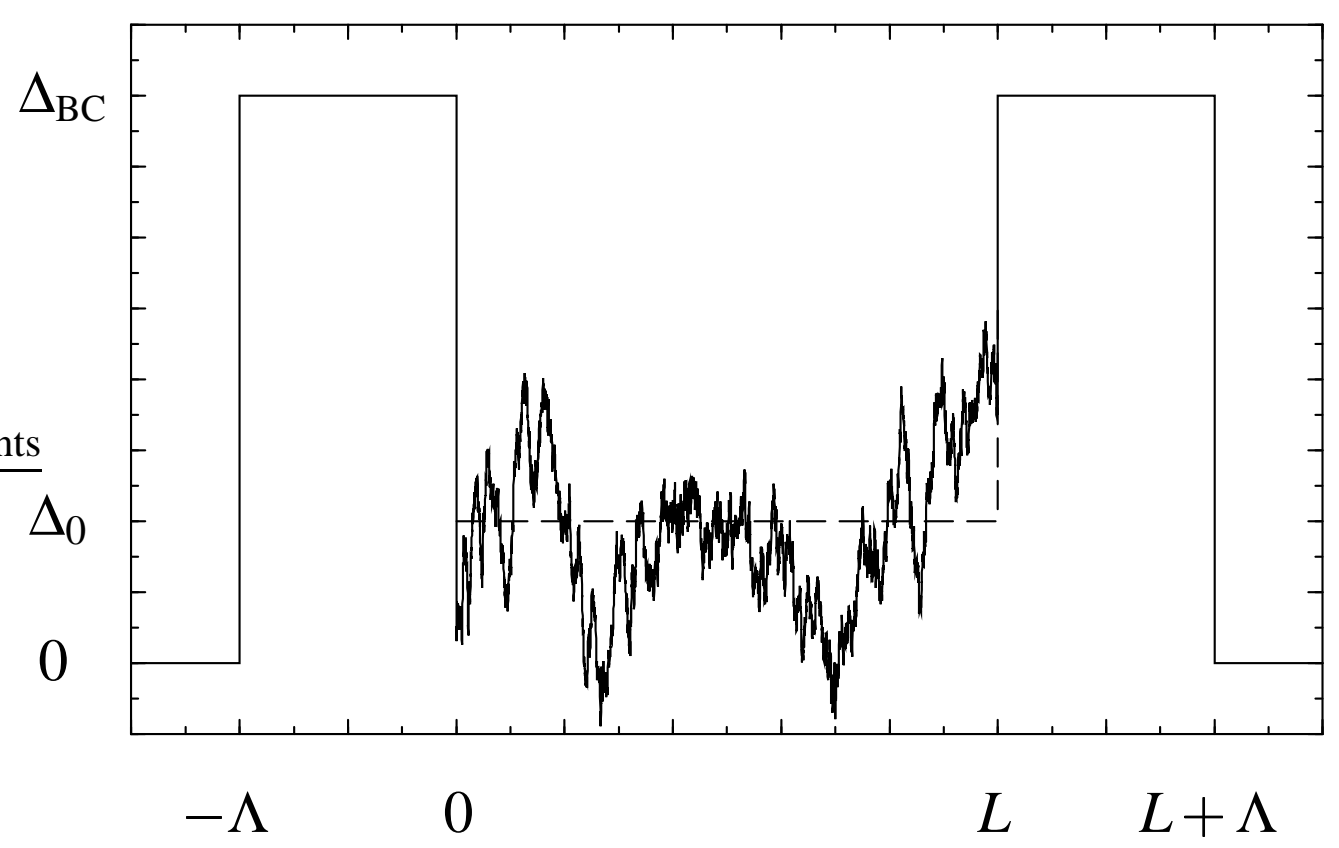

Figure 2.7: Concrete realization of the backscattering potential $\Delta(x)$. While the potential is allowed to fluctuate in the interval $[0, L]$, it is assumed to be equal to $\Delta_{\mathrm{BC}}$ in the intervals $(-\Lambda, 0)$ and $(L, L+\Lambda)$, and outside these intervals $\Delta(x)$ has to vanish.

By definition, $U_{+}(x)=S(x,-\Lambda)$ and $U_{-}(x)=S^{-1}(L+\Lambda, x)$. Because $M^{\dagger}=\sigma_{3} M \sigma_{3}$ and $\operatorname{Tr} M=0$, the $S$-matrices satisfy $S^{\dagger}=\sigma_{3} S^{-1} \sigma_{3}$ and $\operatorname{det} S=1$, which means that they belong to the non-compact group $S U(1,1)$. It follows that the elements of $S$ satisfy $S_{22}=S_{11}^{*}, S_{12}=S_{21}^{*}$, and $\left|S_{11}\right|^{2}-\left|S_{21}\right|^{2}=1$. While each $U_{\alpha}(x)$ only obeys one of the two conditions (2.40), the combination

$$
U(x) \equiv \frac{1}{\sqrt{u}}\left(\begin{array}{cc}
U_{-11}(x) & U_{+12}(x) \\
U_{-21}(x) & U_{+22}(x)
\end{array}\right)
$$

satisfies both boundary conditions. Here, $u=S_{22}(L+\Lambda,-\Lambda)=U_{-11}(-\Lambda)=$ $U_{+22}(L+\Lambda)$, so that $\operatorname{det} U(x)=1$. Defining 2

$$
\begin{aligned}
& \mathbf{u}_{\alpha} \equiv\left(U_{\alpha 11},-U_{\alpha 21}\right)^{T}, \\
& \mathbf{v}_{\alpha} \equiv\left(-U_{\alpha 12}, U_{\alpha 22}\right)^{T},
\end{aligned}
$$

(such that $\mathbf{v}_{\alpha}=\sigma_{1} \mathbf{u}_{\alpha}^{*}$ ), we obtain from Eqs. (2.34), 2.37) and (2.44)

$$
i \mathcal{G}^{R}\left(x, x^{\prime} ; \omega\right)=\theta\left(x-x^{\prime}\right) \frac{\mathbf{u}_{-}(x) \mathbf{u}_{+}^{\dagger}\left(x^{\prime}\right)}{u}+\theta\left(x^{\prime}-x\right) \frac{\mathbf{v}_{+}(x) \mathbf{v}_{-}^{\dagger}\left(x^{\prime}\right)}{u}
$$

$2 \overline{\text { Note, that this definition deviates from the definition used in Ref. [11]. Here, the vectors } \mathbf{u}_{\alpha} \text { and }} \mathbf{v}_{\alpha}$ are given by the first and second column of the matrix $U_{\alpha}$ multiplied from the left by $\pm \sigma_{3}$. 
$\mathbf{u}_{+}^{\dagger}$ and $\mathbf{v}_{-}^{\dagger}$ are the adjungated row vectors to the column vectors $\mathbf{u}_{+}$and $\mathbf{v}_{-}$, so that $\mathbf{u}_{-} \mathbf{u}_{+}^{\dagger}$ and $\mathbf{v}_{+} \mathbf{v}_{-}^{\dagger}$ are $2 \times 2$-matrices. Note that Eq. (2.47) involves only $U_{+12}, U_{+22}$, $U_{-12}^{*}$ and $U_{-22}^{*}$, but not its complex conjugates. In principle, Eq. (2.47) allows to determine the full Green function of the FGM by evaluating time-ordered exponential functions. Equivalent but more complicated forms of this equation were first derived by Abrikosov and Ryzhkin [2]. Of special interest is the trace of the Green function at coinciding space points,

$$
\operatorname{Tr}\left[i \mathcal{G}^{R}(x, x ; \omega)\right]=\frac{U_{+22}(x) U_{-22}^{*}(x)+U_{+12}(x) U_{-12}^{*}(x)}{U_{+22}(x) U_{-22}^{*}(x)-U_{+12}(x) U_{-12}^{*}(x)} .
$$

It immediately follows from Eq. (2.2) that the local DOS is given by

$$
\rho(x, \omega)=\frac{1}{\pi} \operatorname{Re} \frac{U_{+22}(x) U_{-22}^{*}(x)+U_{+12}(x) U_{-12}^{*}(x)}{U_{+22}(x) U_{-22}^{*}(x)-U_{+12}(x) U_{-12}^{*}(x)} .
$$

\subsection{Riccati equation}

Since Eq. 2.49) only depends on the ratios 3

$$
\Phi_{\alpha}(x) \equiv U_{\alpha 12}(x) / U_{\alpha 22}(x),
$$

we may also write

$$
\rho(x, \omega)=\frac{1}{\pi} \operatorname{Re} \frac{1+\Phi_{+}(x) \Phi_{-}^{*}(x)}{1-\Phi_{+}(x) \Phi_{-}^{*}(x)} .
$$

More generally, it follows from Eq. (2.47) that the whole matrix Green function at coinciding space points can be written in terms of $\Phi_{+}(x)$ and $\Phi_{-}^{*}(x)$ :

$$
\begin{aligned}
& i \mathcal{G}^{R}(x, x ; \omega) \equiv \frac{i}{2}\left[\mathcal{G}^{R}\left(x+0^{+}, x ; \omega\right)+\mathcal{G}^{R}\left(x, x+0^{+} ; \omega\right)\right] \\
& \quad=\frac{1}{1-\Phi_{+}(x) \Phi_{-}^{*}(x)}\left(\begin{array}{cc}
\frac{1}{2}\left(1+\Phi_{+}(x) \Phi_{-}^{*}(x)\right) & -\Phi_{+}(x) \\
-\Phi_{-}^{*}(x) & \frac{1}{2}\left(1+\Phi_{+}(x) \Phi_{-}^{*}(x)\right)
\end{array}\right) .
\end{aligned}
$$

Using Eq. (2.39), we find that the $\Phi_{\alpha}(x)$ are both solutions of the same Riccati equation,

$$
-i \partial_{x} \Phi_{\alpha}(x)=2 \tilde{\omega}(x) \Phi_{\alpha}(x)+\Delta(x)+\Delta^{*}(x) \Phi_{\alpha}^{2}(x)
$$

where we have introduced $\tilde{\omega}(x)=\omega-V(x)$. Similar Riccati equations have recently been obtained by Schopohl [82] from the Eilenberger equations of superconductivity. To specify the initial conditions, let us assume that outside the interval $[0, L]$ the potentials $V(x)$ and $\Delta(x)$ are real constants, $V_{\mathrm{BC}}$ and $\Delta_{\mathrm{BC}} \geq 0$. This amounts to taking the limit $\Lambda \rightarrow \infty$, but keeping $L$ constant. The initial values $\Phi_{+}(0)=$ $\lim _{\Lambda \rightarrow \infty} S_{12}(0,-\Lambda) / S_{22}(0,-\Lambda)$ and $\Phi_{-}(L)=\lim _{\Lambda \rightarrow \infty}\left(S^{-1}\right)_{12}(L+\Lambda, L) /\left(S^{-1}\right)_{22}(L+$ $\Lambda, L)$ can be obtained by evaluating the $S$-matrix for constant potentials.

\footnotetext{
${ }^{3}$ Note also that the definition of $\Phi_{\alpha}(x)$ does not involve the extra factor $\pm i$ used in Ref. [11]
} 


\subsubsection{The $S$-matrix for constant potentials}

For constant potentials $V_{n}$ and $\Delta_{n}$, the time-ordering operator $T$ may be omitted in Eq. (2.43), and the $S$-matrix is given by

$$
\begin{gathered}
S_{n}\left(x-x^{\prime}\right)=\exp \left[-i M_{n}\left(x-x^{\prime}\right)\right]=\exp \left[i\left(\tilde{\omega}_{n} \sigma_{3}+\Delta_{n} \sigma_{+}-\Delta_{n} \sigma_{-}\right)\left(x-x^{\prime}\right)\right] \\
=\cosh \left[\sqrt{\left|\Delta_{n}\right|^{2}-\tilde{\omega}_{n}^{2}}\left(x-x^{\prime}\right)\right] \sigma_{0} \\
\quad+i \sinh \left[\sqrt{\left|\Delta_{n}\right|^{2}-\tilde{\omega}_{n}^{2}}\left(x-x^{\prime}\right)\right] \frac{\tilde{\omega}_{n} \sigma_{3}+\Delta_{n} \sigma_{+}-\Delta_{n}^{*} \sigma_{-}}{\sqrt{\left|\Delta_{n}\right|^{2}-\tilde{\omega}_{n}^{2}}} .
\end{gathered}
$$

For $\left|\Delta_{n}\right|^{2}<\tilde{\omega}_{n}^{2}$, the argument of the square root is negative, so that in this case we write the $S$-matrix as 4

$$
\begin{aligned}
S_{n}\left(x-x^{\prime}\right)=\cos & {\left[\sqrt{\tilde{\omega}_{n}^{2}-\left|\Delta_{n}\right|^{2}}\left(x-x^{\prime}\right)\right] \sigma_{0} } \\
& +i \sin \left[\sqrt{\tilde{\omega}_{n}^{2}-\left|\Delta_{n}\right|^{2}}\left(x-x^{\prime}\right)\right] \frac{\widetilde{\omega}_{n} \sigma_{3}+\Delta_{n} \sigma_{+}-\Delta_{n}^{*} \sigma_{-}}{\sqrt{\tilde{\omega}_{n}^{2}-\left|\Delta_{n}\right|^{2}}} .
\end{aligned}
$$

For notational simplicity, let us introduce $\Delta_{n}^{\text {red }} \equiv \sqrt{\left|\Delta_{n}\right|^{2}-\tilde{\omega}_{n}^{2}}$. To calculate $\Phi_{+}(0)$ and $\Phi_{-}(L)$, we need the ratio

$$
\frac{S_{n 12}(x)}{S_{n 22}(x)}=\frac{i \Delta_{n} \Delta_{n}^{\mathrm{red}} \sinh \left[\Delta_{n}^{\mathrm{red}} x\right] \cosh \left[\Delta_{n}^{\mathrm{red}} x\right]-\Delta_{n} \tilde{\omega}_{n} \sinh ^{2}\left[\Delta_{n}^{\mathrm{red}} x\right]}{\left|\Delta_{n}\right|^{2} \cosh ^{2}\left[\Delta_{n}^{\mathrm{red}} x\right]-\tilde{\omega}_{n}^{2}} .
$$

The limit $x \rightarrow \pm \infty$ may now be taken. We obtain

$$
\lim _{x \rightarrow \pm \infty} \frac{S_{n 12}(x)}{S_{n 22}(x)}=\frac{ \pm i \Delta_{n}^{\text {red }}-\tilde{\omega}_{n}}{\Delta_{n}^{*}} .
$$

If we keep in mind that the frequency $\omega$ involves a small imaginary part, we see that this result is not restricted to the case $\left|\Delta_{n}\right|^{2}>\tilde{\omega}_{n}^{2}$ : For $\left|\Delta_{n}\right|^{2} \leq \tilde{\omega}_{n}^{2}$ the square root has to be taken such that the right-hand side of Eq. (2.57) vanishes as $\Delta_{n} \rightarrow 0$. This follows directly from the definition of the $S$-matrix.

\subsubsection{Initial conditions}

It follows from Eq. (2.57) that the Riccati equation 2.53) should be integrated with the initial conditions

$$
\Phi_{+}(0)=\Phi_{-}^{*}(L)= \begin{cases}\frac{i \sqrt{\Delta_{\mathrm{BC}}^{2}-\left(\omega-V_{\mathrm{BC}}\right)^{2}}-\left(\omega-V_{\mathrm{BC}}\right)}{\Delta_{\mathrm{BC}}} & , \Delta_{\mathrm{BC}}^{2}>\left(\omega-V_{\mathrm{BC}}\right)^{2}, \\ \frac{\operatorname{sgn}\left(\omega-V_{\infty}\right) \sqrt{\left(\omega-V_{\mathrm{BC}}\right)^{2}-\Delta_{\mathrm{BC}}^{2}}-\left(\omega-V_{\mathrm{BC}}\right)}{\Delta_{\mathrm{BC}}} & , \Delta_{\mathrm{BC}}^{2} \leq\left(\omega-V_{\mathrm{BC}}\right)^{2},\end{cases}
$$

\footnotetext{
${ }^{4}$ Note that the transition from Eq. (2.54) to Eq. (2.55) is independent of the definition of the square root.
} 
where $\operatorname{sgn}(x)$ is equal to 1 for positive $x$ and equal to -1 for negative $x$. While for $\Delta_{\mathrm{BC}}=0$ the initial conditions are given by $\Phi_{+}(0)=\Phi_{-}^{*}(L)=0$, for $\Delta_{\mathrm{BC}} \rightarrow \infty$ one gets $\Phi_{+}(0)=\Phi_{-}^{*}(L)=i$. Note that for arbitrary potentials $V_{\mathrm{BC}}$ and $\Delta_{\mathrm{BC}}$, the initial values are simply given by the stable stationary solution of the Riccati equation (2.53) with $V(x)=V_{\mathrm{BC}}$ and $\Delta(x)=\Delta_{\mathrm{BC}}$.

\subsubsection{The case of a discrete spectrum}

Defining the (complex) phase $\varphi_{\alpha}(x)$ via

$$
\Phi_{\alpha}(x)=e^{i \varphi_{\alpha}(x)}
$$

and decomposing $\Delta(x)$ into its amplitude $|\Delta(x)|$ and its phase $\vartheta(x)$,

$$
\Delta(x)=|\Delta(x)| e^{i \vartheta(x)}
$$

the Riccati equation 2.53), turns into

$$
\partial_{x} \varphi_{\alpha}(x)=2 \tilde{\omega}(x)+2|\Delta(x)| \cos \left[\varphi_{\alpha}(x)-\vartheta(x)\right]
$$

Note that this equation of motion is of the Langevin type [34, 46]

$$
\partial_{x} v(x)=-a+b_{1} V(x)+b_{2}^{*}(v) \Delta(x)+b_{2}(v) \Delta^{*}(x) .
$$

Let us consider the case $\left(\omega-V_{\mathrm{BC}}\right)^{2}<\Delta_{\mathrm{BC}}^{2}$ : It directly follows from Eq. (2.58) that $\left|\Phi_{+}(0)\right|=\left|\Phi_{-}(L)\right|=1$, such the initial values $\varphi_{+}(0)$ and $\varphi_{-}(L)$ are real. Hence, the solutions of Eq. (2.61) remain real, which implies $\left|\Phi_{\alpha}(x)\right|=1$ for all $x$. As can be seen from Eq. (2.58), $\varphi_{+}(0)$ and $\varphi_{-}(L)$ can be chosen to fulfill $\varphi_{+}(0)=-\varphi_{-}(L) \in[0, \pi]$, so that the initial values $\varphi_{+}(0)$ and $\varphi_{-}(L)$ are uniquely determined by

$$
\cot \varphi_{+}(0)=-\cot \varphi_{-}(L)=-\frac{\omega-V_{\mathrm{BC}}}{\sqrt{\Delta_{\mathrm{BC}}^{2}-\left(\omega-V_{\mathrm{BC}}\right)^{2}}} .
$$

For the phases $\varphi_{\alpha}(x)$ to be continuous, they have to be unreduced phases which are not limited to take values between 0 and $2 \pi$. In terms of the $\varphi_{\alpha}(x)$, the local DOS can be written as

$$
\begin{aligned}
\rho(x, \omega) & =-\frac{1}{\pi} \operatorname{Im} \cot \left[\frac{\varphi_{+}(x)-\varphi_{-}(x)}{2}+i 0\right] \\
& =2 \sum_{m=-\infty}^{\infty} \delta\left(\varphi_{+}(x)-\varphi_{-}(x)-2 \pi m\right) .
\end{aligned}
$$

It is easy to show that for $x \geq 0$ the phase $\varphi_{+}(x, \omega)$ is a monotonic increasing function of $\omega$ : For the initial value at $x=0$, this follows directly from Eq. (2.63),

$$
\partial_{\omega} \varphi_{+}(0)=-\partial_{\omega} \varphi_{-}(L)=\frac{1}{\sqrt{\Delta_{\mathrm{BC}}^{2}-\left(\omega-V_{\mathrm{BC}}\right)^{2}}}>0 .
$$


It can be seen directly from Eq. (2.61) that $\partial_{\omega} \varphi_{+}(x)>0$ is true for every $x \geq 0$. More formally, differentiating the equation of motion (2.61) with respect to $\omega$ gives

$$
\partial_{x} \partial_{\omega} \varphi_{\alpha}(x, \omega)=2-2|\Delta(x)| \sin \left[\varphi_{\alpha}(x)-\vartheta(x)\right] \partial_{\omega} \varphi_{\alpha}(x, \omega) \text {. }
$$

Solving this first-order differential equation for $\partial_{\omega} \varphi_{\alpha}(x, \omega)$, we obtain

$$
\begin{aligned}
& \partial_{\omega} \varphi_{\alpha}(x, \omega)=\partial_{\omega} \varphi_{\alpha}\left(x_{0}, \omega\right) \\
& \quad+2 \int_{x_{0}}^{x} \exp \left[-2 \int_{x^{\prime}}^{x}\left|\Delta\left(x^{\prime \prime}\right)\right| \sin \left[\varphi_{\alpha}\left(x^{\prime \prime}\right)-\vartheta\left(x^{\prime \prime}\right)\right] d x^{\prime \prime}\right] d x^{\prime}
\end{aligned}
$$

Since both terms on the right-hand side of Eq. (2.67) are positive for $\alpha=+$ and $x_{0}=0$, we have

$$
\partial_{\omega} \varphi_{+}(x, \omega)>0 \text { for } x \geq 0 .
$$

Analogously we find

$$
\partial_{\omega} \varphi_{-}(x, \omega)<0 \text { for } x \leq L .
$$

For arbitrary $\omega, \varphi_{+}(x, \omega)$ integrated with the initial condition $\varphi_{+}(0, \omega)$ given in Eq. (2.63) will usually not be equal to $\varphi_{-}(L, \omega)$ up to a multiple of $2 \pi$ at $x=L$. For certain discrete frequencies $\omega_{m}$, however, this is the case. Since $\varphi_{+}(x, \omega)$ is a monotonic function of $\omega$, we can uniquely define $\omega_{m}$ by the condition

$$
\varphi_{+}\left(L, \omega_{m}\right)=\varphi_{-}\left(L, \omega_{m}\right)+2 \pi m .
$$

Note that $\omega_{m}$ is only well-defined if it turns out that $\left(\omega_{m}-V_{\mathrm{BC}}\right)^{2}<\Delta_{\mathrm{BC}}^{2}$. Since the right-hand side of Eq. (2.61) is a $2 \pi$-periodic function of $\varphi_{\alpha}(x)$, we see that $\varphi_{+}\left(x, \omega_{m}\right)$ and $\varphi_{-}\left(x, \omega_{m}\right)$ are equal up to the constant $2 \pi m$ for every $x \in[0, L]$,

$$
\varphi_{+}\left(x, \omega_{m}\right)-\varphi_{-}\left(x, \omega_{m}\right)=2 \pi m .
$$

Of course, the $\omega_{m}$ are the discrete eigenvalues of the system. This follows immediately from the fact that the local DOS $\rho(x, \omega)$ is equal to zero if not $\omega=\omega_{m}$ for one $m$. Therefore, the total DOS is given by

$$
\rho(\omega)=\frac{1}{L} \sum_{m} \delta\left(\omega-\omega_{m}\right)
$$

Using the well-known formula

$$
\delta(f(\omega))=\sum_{m} \frac{1}{\left|f^{\prime}\left(\omega_{m}\right)\right|} \delta\left(\omega-\omega_{m}\right)
$$

where $\omega_{m}$ are the zeros of $f(\omega)$, we can write Eq. (2.64) as

$$
\rho(x, \omega)=\sum_{m} \frac{2 \delta\left(\omega-\omega_{m}\right)}{\left|\partial_{\omega} \varphi_{+}(x, \omega)-\partial_{\omega} \varphi_{-}(x, \omega)\right|} .
$$


Expressing $\varphi_{\alpha}(x, \omega)$ by the right-hand side of Eq. (2.67) with $\alpha=+$ and $x_{0}=0$ or $\alpha=-$ and $x_{0}=L$, respectively, we arrive at

$$
\rho(x, \omega)=\sum_{m} \frac{\delta\left(\omega-\omega_{m}\right)}{\int_{0}^{L} d x^{\prime} \exp \left(-2 \int_{x^{\prime}}^{x} d x^{\prime \prime}\left|\Delta\left(x^{\prime \prime}\right)\right| \sin \left[\varphi_{\alpha}\left(x^{\prime \prime}, \omega_{m}\right)-\vartheta\left(x^{\prime \prime}\right)\right]\right)} .
$$

Once the eigenvalues $\omega_{m}$ have been determined, this equation in principle allows to calculate the local DOS for arbitrary potentials $V(x)$ and $\Delta(x)$.

\subsection{Integrated averaged Green function $\Gamma(\omega)$}

In the last subsection, we have seen that the integrated DOS can be obtained by solving a simple initial value problem for the phase $\varphi(x)$, which is a functional of the disorder. To implement the correct boundary conditions for a system of length $L$, we have first assumed that outside the interval $(0, L)$ the potentials are constant over a range $\Lambda$, but then drop to zero. Finally, we have let $\Lambda$ go to infinity. However, if we are only interested in the limit $L \rightarrow \infty$, i.e. the bulk properties, we can set $\Lambda=0$ at the beginning of our calculations. The physical meaning of this is that bulk properties should be independent of the boundary conditions.

By setting the potential equal to zero outside the interval $(0, L)$, we will not only be able to recover the equation of motion (2.61) satisfied by the phase $\varphi(x)$ which determines the integrated DOS, we will also be able to derive an additional equation which allows to calculate the inverse localization length.

It follows from Eq. (2.48) that the trace of the space-averaged diagonal element of the retarded Green function is given by

$$
\begin{aligned}
\langle\operatorname{Tr} & {\left.\left[\mathcal{G}^{R}(x, x ; \omega)\right]\right\rangle_{x} \equiv \frac{1}{L} \int_{0}^{L} d x \operatorname{Tr}\left[\mathcal{G}^{R}(x, x ; \omega)\right] } \\
& =\frac{1}{L S_{22}(L, 0)}\left[-i \int_{0}^{L} d x\left(S_{22}(L, x) S_{22}(x, 0)-S_{21}(L, x) S_{12}(x, 0)\right)\right] .
\end{aligned}
$$

The term in angular brackets can easily be identified to be equal to $\partial_{\omega} S_{22}(L, 0)$ :

$$
\begin{aligned}
\partial_{\omega} S_{22}(L, 0) & =\left(\partial_{\omega} T \exp \left[-i \int_{0}^{L} d x^{\prime} M\left(x^{\prime}\right)\right]\right)_{22} \\
& =\left(\int_{0}^{L} d x S(L, x)\left[-i \partial_{\omega} M(x)\right] S(x, 0)\right)_{22} \\
& =i \int_{0}^{L} d x\left(S(L, x) \sigma_{3} S(x, 0)\right)_{22} \\
& =-i \int_{0}^{L} d x\left(S_{22}(L, x) S_{22}(x, 0)-S_{21}(L, x) S_{12}(x, 0)\right) .
\end{aligned}
$$

We can therefore rewrite Eq. (2.76) as

$$
\left\langle\operatorname{Tr}\left[\mathcal{G}^{R}(x, x ; \omega)\right]\right\rangle_{x}=\frac{\partial_{\omega} S_{22}(L, 0)}{L S_{22}(L, 0)}=\frac{1}{L} \partial_{\omega} \ln \left[S_{22}(L, 0)\right]
$$


To describe the bulk properties, one should now take the limit $L \rightarrow \infty$. Since this expression involves the partial derivative with respect to $\omega$, we introduce the trace of the energy-integrated space-averaged Green function at coinciding space points,

$$
\Gamma(\omega) \equiv \lim _{L \rightarrow \infty} \frac{1}{L} \ln \left[S_{22}(L, 0 ; \omega)\right]
$$

so that

$$
\left\langle\operatorname{Tr}\left[\mathcal{G}^{R}(x, x ; \omega)\right]\right\rangle=\partial_{\omega} \Gamma(\omega) .
$$

While the integrated DOS is given by $\mathcal{N}(\omega)=-\pi^{-1} \operatorname{Im} \Gamma(\omega)$, we will see in the next section that $\operatorname{Re} \Gamma(\omega)$ is equal to the inverse localization length $\ell^{-1}(\omega)$. The decomposition of $\Gamma(\omega)$ into its real and imaginary part can therefore be written as

$$
\Gamma(\omega)=\ell^{-1}(\omega)-i \pi \mathcal{N}(\omega)
$$

Both $\mathcal{N}(\omega)$ and $\ell^{-1}(\omega)$ can simultaneously be calculated by determining the logarithm of the $S$-matrix element $S_{22}$. It is convenient to express the $S$-matrix elements in terms of their phases. Let us define $\varphi_{\alpha \alpha^{\prime}}(x)$ via

$$
S_{\alpha \alpha^{\prime}}(x, 0) \equiv e^{-i \varphi_{\alpha \alpha^{\prime}}(x)} .
$$

$\Gamma(\omega)$ is then given by

$$
i \Gamma(\omega)=\lim _{L \rightarrow \infty} \frac{\varphi_{22}(L)}{L} .
$$

Introducing $\bar{\alpha} \equiv-\alpha$, the properties of the $S$-matrix $S_{\alpha \alpha^{\prime}}=S_{\bar{\alpha} \bar{\alpha}^{\prime}}^{*}$ render into $\varphi_{\alpha \alpha^{\prime}}=$ $-\varphi_{\bar{\alpha} \bar{\alpha}^{\prime}}^{*}$. The $S$-matrix can be expressed in terms of $\varphi_{12}, \varphi_{22}$ and its complex conjugates 5 It follows from $i \partial_{x} S(x, 0)=M(x) S(x, 0)$ that the $\varphi_{\alpha \alpha^{\prime}}$ satisfy

$$
\partial_{x} \varphi_{\alpha \alpha^{\prime}}(x)=M_{\alpha \alpha}(x)+M_{\alpha \bar{\alpha}}(x) \exp \left[i\left(\varphi_{\alpha \alpha^{\prime}}(x)-\varphi_{\bar{\alpha} \alpha^{\prime}}(x)\right)\right] .
$$

Recalling that $M(x)=-\tilde{\omega}(x) \sigma_{3}-\Delta(x) \sigma_{+}+\Delta^{*}(x) \sigma_{-}$, the two equations for $\varphi_{12}$ and $\varphi_{22}$ read

$$
\begin{aligned}
& \partial_{x} \varphi_{22}(x)=\tilde{\omega}(x)+\Delta^{*}(x) \exp \left[i\left(\varphi_{22}(x)-\varphi_{12}(x)\right)\right], \\
& \partial_{x} \varphi_{12}(x)=-\tilde{\omega}(x)-\Delta(x) \exp \left[-i\left(\varphi_{22}(x)-\varphi_{12}(x)\right)\right] .
\end{aligned}
$$

If we now introduce

$$
\begin{aligned}
& \varphi(x) \equiv \varphi_{22}(x)-\varphi_{12}(x) \\
& \zeta(x) \equiv-i\left(\varphi_{22}(x)+\varphi_{12}(x)\right)
\end{aligned}
$$

5 Recall that instead of $\alpha, \alpha^{\prime}=+,-$ we also use $i, j=1,2$ (see footnote 1 on page 37 
we arrive at the following system of equations of motion:

$$
\begin{aligned}
& \partial_{x} \varphi(x)=2 \tilde{\omega}(x)+2|\Delta(x)| \cos [\varphi(x)-\vartheta(x)] \\
& \partial_{x} \zeta(x)=2|\Delta(x)| \sin [\varphi(x)-\vartheta(x)]
\end{aligned}
$$

Note that Eq. (2.89), which determines $\varphi(x)$, is exactly the same Langevin equation that we derived before from the Riccati equation [see Eq. 2.61] and is independent from Eq. (2.90). After having found a solution to Eq. (2.89), $\zeta(x)$ in principle can be obtained by integrating Eq. (2.90). In terms of $\varphi(x)$ and $\zeta(x), \Gamma(\omega)$ is now given by

$$
i \Gamma(\omega)=\lim _{L \rightarrow \infty}[\varphi(L)+i \zeta(L)] / 2 L .
$$

The initial condition $S(0,0)=\sigma_{0}$ could be mapped on the initial conditions for $\varphi(0)$ and $\zeta(0)$ which are, strictly speaking, singular but integrable. However, since $\Gamma(\omega)$ is determined by the asymptotics for large $L$, the initial conditions finally drop out in the limit $L \rightarrow \infty$. We can therefore also choose $\varphi(0)=\zeta(0)=0$, such that $\varphi(x)$ and $\zeta(x)$ are real for all $x$ and there is no finite initial value which as $L$ becomes large only dies out as $1 / L$. With these initial conditions, we can describe the bulk properties of the system right from the beginning. The integrated DOS and the inverse localization length can now be expressed as

$$
\begin{aligned}
\mathcal{N}(\omega) & =\rho_{0} \lim _{L \rightarrow \infty} \varphi(L) / 2 L, \\
\ell^{-1}(\omega) & =\lim _{L \rightarrow \infty} \zeta(L) / 2 L .
\end{aligned}
$$

These two equations in combination with the equations of motion (2.89) and (2.90) allow for simultaneous exact numerical computations of the (integrated) DOS and the inverse localization length for arbitrary given disorder potentials. Since the (integrated) DOS and the inverse localization length are self-averaging quantities [58], it is sufficient to consider just one typical realization of the disorder potential. We will do this for various interesting cases in Chapter 4.

In analytical calculations one does not usually work with a certain realization of the disorder. Instead, one tries to calculate averaged quantities by using the given statistical properties of the disorder potentials. Taking the average of Eq. (2.91) with respect to the distribution of the random potentials $V(x)$ and $\Delta(x)$, we obtain

$$
i\langle\Gamma(\omega)\rangle=\lim _{L \rightarrow \infty}[\langle\varphi(L)\rangle+i\langle\zeta(L)\rangle] / 2 L .
$$

Integrating the equations of motion (2.89) and 2.90) with respect to $x$ from 0 to $L$, $\langle\Gamma(\omega)\rangle$ can be rewritten as

$$
i\langle\Gamma(\omega)\rangle=\lim _{L \rightarrow \infty} \frac{1}{L} \int_{0}^{L} d x\left\langle\tilde{\omega}(x)+\Delta^{*}(x) \exp [i \varphi(x)]\right\rangle .
$$


Instead of looking at $\left\langle\tilde{\omega}(x)+\Delta^{*}(x) \exp [i \varphi(x)]\right\rangle$ as an average with respect to the probability distribution involving the disorder at $x$ and via $\varphi(x)$ also at all space points between 0 and $x$, we can also consider the average to be with respect to the joint probability distribution of the random potentials at $x$ and the unreduced phase $\varphi(x)$. The process of averaging has now become local. Since $\exp [i \varphi(x)]$ is a $2 \pi$-periodic function in $\varphi(x)$, it is also sufficient to use the joint probability distribution of the random potentials and the reduced phase $\varphi(x) \in[0,2 \pi)$. While the joint probability distribution of the random potential and the non-reduced phase is only well-defined for finite $x$ (and, of course, depends on $x$ ), the joint probability distribution of the random potential and the reduced phase becomes independent of $x$ for large $x$ and therefore in this limit is equal to the stationary probability distribution. Recalling that $\langle V(x)\rangle=0$, Eq. (2.95) reduces to

$$
i\langle\Gamma(\omega)\rangle=\omega+\left\langle\Delta^{*}(x) \exp [i \varphi(x)]\right\rangle
$$

This equation expresses the averaged integrated DOS as well as the averaged inverse localization length in terms of a relatively simple function averaged over the joint stationary distribution of the random potentials $V(x), \Delta(x)$ and the reduced phase $\varphi(x)$. Recall that $\Delta(x)$ is allowed to be complex. We will use Eq. (2.96) in Chapter 3 to calculate both $\mathcal{N}(\omega)$ and $\ell^{-1}(\omega)$ for the FGM in the white noise limit. In principle, Eq. (2.96) also applies to less restrictive cases.

\subsection{Gauge invariance}

It turns out that fluctuations of the forward scattering disorder have similar effects on the DOS and localization length as have phase fluctuations of the gap parameter. To illuminate the hidden symmetry, let us again consider the equation satisfied by the retarded Green function $\mathcal{G}^{R}\left(x, x^{\prime} ; \omega\right)$,

$$
\left(\begin{array}{cc}
\omega-V(x)+i \partial_{x} & -\Delta(x) \\
-\Delta^{*}(x) & \omega-V(x)-i \partial_{x}
\end{array}\right) \mathcal{G}^{R}\left(x, x^{\prime} ; \omega\right)=\delta\left(x-x^{\prime}\right) \sigma_{0} .
$$

A crucial point of this equation is that its form is left invariant under the gauge transformation [16]

$$
\begin{aligned}
\mathcal{G}^{R}\left(x, x^{\prime} ; \omega\right) & \rightarrow \exp \left[-(i / 2) \chi(x) \sigma_{3}\right] \mathcal{G}^{R}\left(x, x^{\prime} ; \omega\right) \exp \left[(i / 2) \chi\left(x^{\prime}\right) \sigma_{3}\right], \\
V(x) & \rightarrow V(x)+\frac{1}{2} \partial_{x} \chi(x) \\
\Delta(x) & \rightarrow \Delta(x) \exp [-i \chi(x)] .
\end{aligned}
$$

Here, $\chi(x)$ is a local phase rotation which is allowed to vary arbitrarily from point to point. Since the trace of a product of matrices is invariant under cyclic permutations of the matrices, $\operatorname{Tr} \mathcal{G}^{R}(x, x ; \omega)$ and therefore the local DOS and the inverse localization length 6 are also invariants under the above gauge transformation.

\footnotetext{
${ }^{6}$ Strictly speaking, it follows only that $\partial_{\omega} \ell^{-1}(\omega)$ is left invariant. The integration constants at $\omega=\infty$ are, however, the same, so that $\ell^{-1}(\omega)$ is invariant under the gauge transformation.
} 
Instead of directly looking at the trace of the Green function at coinciding space points, we can also consider the equations of motion (2.89) and (2.90). Their form is gauge invariant under the combined transformation

$$
\begin{aligned}
\varphi(x) & \rightarrow \varphi(x)-\chi(x) \\
\zeta(x) & \rightarrow \zeta(x) \\
V(x) & \rightarrow V(x)+\frac{1}{2} \partial_{x} \chi(x) \\
\vartheta(x) & \rightarrow \vartheta(x)-\chi(x)
\end{aligned}
$$

It follows from Eqs. (2.92) and (2.93) that both the integrated DOS $\mathcal{N}(\omega)$ and the inverse localization length $\ell^{-1}(\omega)$ are invariant under the considered gauge transfor-

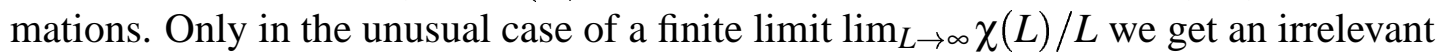
shift in the additive constant of $\mathcal{N}(\omega)$.

Besides $\chi(x) \equiv 0$, the choices for which either $V(x)$ or $\vartheta(x)$ vanishes are especially convenient.

1. Effectively vanishing phase fluctuations: If $\vartheta(x)$ is differentiable, we can define

$$
\chi(x) \equiv \vartheta(x)
$$

such that the forward scattering potential is modified in such a way that there are no phase fluctuations of $\Delta(x)$ left. In this gauge we have to let

$$
\begin{aligned}
& V(x) \rightarrow V(x)+\frac{1}{2} \partial_{x} \vartheta(x), \\
& \Delta(x) \rightarrow|\Delta(x)| .
\end{aligned}
$$

Note that in this gauge the order parameter field $|\Delta(x)|$ is real and positive. We will use the above gauge transformation at the end of Chapter 4 to find an exact solution for the FGM involving only phase fluctuations.

2. Effectively vanishing forward scattering potential: Choosing the phase $\chi(x)$ such that

$$
\frac{1}{2} \partial_{x} \chi(x)=-V(x)
$$

the forward scattering potential $V(x)$ can be eliminated by renormalizing the phase fluctuations $\vartheta(x)$ of the backscattering potential $\Delta(x)$. Explicitly, we let

$$
\begin{aligned}
& V(x) \rightarrow 0 \\
& \Delta(x) \rightarrow \Delta(x) \exp \left[i 2 \int_{x_{0}}^{x} V\left(x^{\prime}\right) d x^{\prime}\right] .
\end{aligned}
$$




\subsection{Lyapunov exponent and localization length}

In this section, we will explicitly show that the Thouless formula holds for the FGM and $\operatorname{Re} \Gamma(\omega)$ can indeed be identified with the inverse localization length $\ell^{-1}(\omega)$.

One of the striking properties of disordered systems treated in the independent electron approximation is the fact that the disorder can lead to a macroscopic large number of localized states. These states are eigenfunction of the Schrödinger equation falling off exponentially with distance from one point in space which is characteristic for the particular solution. Non-localized states only exist between two mobility edges, and as the strength of the disorder increases, the width of this energy region narrows. Finally, as both mobility edges coincide, all states become localized and the conductor turns into an insulator. This phase transition is known as the Anderson transition [5].

In one dimension, an arbitrary weak disorder suffices to localize all eigenstates (excluding perhaps states at isolated energy values) [69] of electrons treated in the independent electron approximation. As a consequence the diffusion coefficient and the dc conductivity vanish. Therefore, there is no metal-insulator transition in one dimension.

\subsubsection{Thouless formula}

Since the energy dispersion of the FGM is linear, the Schrödinger equation of the FGM, $\hat{H} \psi(x)=\omega \psi(x)$, is a linear first order differential equation. Fixing the twocomponent wave function $\psi(x) \equiv\left(\psi_{1}(x), \psi_{2}(x)\right)^{T}$ at one space point $x_{0}$ therefore constitutes the wave function at all space points $x$. As we will see below, for large distances $\left|x-x_{0}\right|$ the envelope of the wave function will grow exponentially with probability one, i.e. ||$\psi(x) \| \sim|| \psi_{0}|| \exp \left(+\gamma\left|x-x_{0}\right|\right)$, where ||$\psi(x) \|^{2} \equiv\left|\psi_{1}(x)\right|^{2}+\left|\psi_{2}(x)\right|^{2}$. Of course, $\psi(x)$ cannot be an eigenfunction of the Hamiltonian $\hat{H}$ satisfying the right boundary conditions. The proportionality factor $\gamma$ in the exponential function is called the Lyapunov exponent. The (mean) localization length is usually defined to be equal to the inverse Lyapunov exponent [58]. This definition can be motivated by considering two wave functions of a large but finite system which are fixed at the left or right end of the sample, respectively. Both solutions grow exponentially as one moves into the bulk and usually they do not match. For certain discrete energy values $\omega_{m}$, however, the wave functions can be matched after an appropriate rescaling and one obtains an eigenfunction to $\hat{H}$ obeying the correct boundary conditions. Of course, the resulting wave function can not simultaneously grow and fall off. Instead, one expects the envelope of the wave function to have a maximum somewhere in the bulk. One also expects the wave function to fall off exponentially from this point $x_{m}$ such that ||$\psi(x)\|\sim\| \psi_{m}|| \exp \left(-\gamma\left|x-x_{m}\right|\right)$. Defining the localization length to be equal to the inverse of the Lyapunov exponent $\gamma$ therefore seems to be a reasonable definition. 
More explicitly, we define

$$
\ell^{-1}(\omega) \equiv \gamma(\omega) \equiv \lim _{L \rightarrow \infty} \frac{1}{L} \ln \left(\frac{\|\psi(L)\|}{\left\|\psi_{0}\right\|}\right) .
$$

Note that since we take the limit $L \rightarrow \infty$, the initial value $\left\|\psi_{0}\right\|$ drops out.

Let us now rewrite the Schrödinger equation such that the differential operator $\partial_{x}$ is proportional to the unit matrix. As in the non-Abelian Schwinger-ansatz we factor out a $\sigma_{3}$-matrix so that the wave function $\tilde{\psi}(x) \equiv \sigma_{3} \psi(x)$ satisfies

$$
\left[i \partial_{x}-M(x)\right] \tilde{\psi}(x)=0 .
$$

Here, $M(x)$ is the matrix defined in Eq. (2.36). The solution to the Schrödinger equation (2.112) is therefore given by

$$
\tilde{\psi}(x)=S\left(x, x_{0}\right) \tilde{\psi}_{0} \text { • }
$$

It follows with $\|\tilde{\psi}(x)\|=\|\psi(x)\|$ that the Lyapunov exponent can be expressed in terms of the $S$-matrix,

$$
\gamma(\omega)=\lim _{L \rightarrow \infty} \frac{1}{L} \ln \left\|S(L, 0) \tilde{\psi}_{0}\right\| .
$$

It is now easy to see that with probability one, $\gamma(\omega)$ does not depend on $\tilde{\psi}_{0}$ : Let $s_{ \pm}(x)$ be the two eigenvalues of $S(x, 0)$, where $\left|s_{+}(x)\right|>\left|s_{-}(x)\right|=\left|s_{+}(x)\right|^{-1}$. If $\tilde{\psi}_{+}(x)$ and $\tilde{\psi}_{-}(x)$ are the coefficients of the vector $\tilde{\psi}(x)$ in the corresponding eigenbasis, we can rewrite Eq. (2.114) as

$$
\gamma(\omega)=\lim _{L \rightarrow \infty} \frac{1}{L} \ln \left(\left|\tilde{\Psi}_{+}(L) s_{+}(L)\right|^{2}+\left|\tilde{\Psi}_{-}(L) s_{-}(L)\right|^{2}\right)^{1 / 2}
$$

As long as the coefficient $\tilde{\psi}_{+}(L)$ of the exponentially increasing solution of the Schrödinger equation does not vanish (and this should be the case with probability one), Eq. 2.115) reduces to

$$
\gamma(\omega)=\lim _{L \rightarrow \infty} \frac{1}{L} \ln \left|s_{+}(L)\right|
$$

$\gamma(\omega)$ is clearly independent of the initial wave function $\tilde{\psi}_{0}$ and only depends on the largest eigenvalue of the $S$-matrix which grows exponentially as the length of the system increases. Choosing $\tilde{\psi}_{0}=(0,1)^{T}$, it follows from Eq. (2.114)

$$
\gamma(\omega)=\lim _{L \rightarrow \infty} \frac{1}{L} \ln \left(\left|S_{12}(L, 0)\right|^{2}+\left|S_{22}(L, 0)\right|^{2}\right)^{1 / 2}
$$

which

due to $\ln \left(\left|S_{12}(L, 0)\right|^{2}+\left|S_{22}(L, 0)\right|^{2}\right)^{1 / 2}=\ln \left(2\left|S_{22}(L, 0)\right|^{2}-1\right)^{1 / 2} \sim \ln \left|S_{22}(L, 0)\right|$ simplifies to

$$
\gamma(\omega)=\lim _{L \rightarrow \infty} \frac{1}{L} \ln \left|S_{22}(L, 0)\right| .
$$


Comparing this equation with Eq. (2.79), we see that the inverse localization length $\ell^{-1}(\omega) \equiv \gamma(\omega)$ is in fact equal to $\operatorname{Re} \Gamma(\omega)$. The equation

$$
\ell^{-1}(\omega)=\operatorname{Re} \Gamma(\omega)
$$

is known as the Thouless formula [91] and was first shown to be valid for the FGM by Hayn and John [40] in a different way. But note that these authors have only shown Eq. (2.119) up to an integration constant which they had to determine by different means.

\subsubsection{Localization length at $\omega=0$ for real $\Delta(x)$}

Although we are going to postpone detailed calculations of the DOS and the localization length to the next chapters, let us now consider the localization length at frequency $\omega=0$ for a real disorder potential $\Delta(x)$ and $V(x)=0$. In this case $M(x)=-i \sigma_{2} \Delta(x)$, so that the $S$-matrix may be expressed without the path-ordering operator as

$$
\begin{aligned}
S\left(x, x^{\prime}\right) & =\exp \left[-\sigma_{2} \int_{x^{\prime}}^{x} \Delta(y) d y\right] \\
& =\cosh \left[\int_{x^{\prime}}^{x} \Delta(y) d y\right] \sigma_{0}-\sinh \left[\int_{x^{\prime}}^{x} \Delta(y) d y\right] \sigma_{2} .
\end{aligned}
$$

It directly follows from Eq. (2.118) that the inverse localization length at $\omega=0$ is given by

$$
\begin{aligned}
\ell^{-1}(0) & =\lim _{L \rightarrow \infty} \frac{1}{L} \ln \left|S_{22}(L, 0)\right|=\lim _{L \rightarrow \infty} \frac{1}{L} \ln \left(\cosh \left[\int_{0}^{L} \Delta(y) d y\right]\right) \\
& =\left|\lim _{L \rightarrow \infty} \frac{1}{L} \int_{0}^{L} \Delta(y) d y\right| \equiv\left|\langle\Delta(x)\rangle_{x}\right|=\left|\Delta_{\mathrm{av}}\right| .
\end{aligned}
$$

where we have used the fact that in the limit $L \rightarrow \infty$, the average $\langle\Delta(x)\rangle_{x}$ is equal to the expectation value $\Delta_{\mathrm{av}}$. The inverse localization length at frequency $\omega=0$ is equal to the absolute value of the expectation value of the backscattering potential $\langle\Delta(x)\rangle$ and does not depend on the random fluctuations around this average value. Note that this result is valid for arbitrary higher correlation functions of $\Delta(x)$. In the case $\langle\Delta(x)\rangle=0$, the localization length diverges for $\omega=0$ which clearly distinguishes the point $\omega=0$.

\subsection{Eilenberger and pseudo-Schrödinger equation}

For slowly varying potentials, one might be interested in a gradient expansion of the local DOS which determines the gradient expansion of thermodynamic quantities. For example, a gradient expansion of the free energy allows for a microscopic derivation of the Ginzburg-Landau free energy functional and corrections to it. We will now 
deduce an equation which in the semiclassical theory of superconductivity is known as the Eilenberger equation [21, 56]. We will also derive a related pseudo-Schrödinger equation. Both equations can be used to recursively calculate all orders in the gradient expansion of the Green function at coinciding space points. Consider the Eilenberger function

$$
\tilde{g}^{R}(x, \omega) \equiv \sigma_{3} \mathcal{G}^{R}(x, x, \omega)=-i \frac{\sigma_{3} \mathbf{u}_{-}(x) \mathbf{u}_{+}^{\dagger}(x)+\sigma_{3} \mathbf{v}_{+}(x) \mathbf{v}_{-}^{\dagger}(x)}{2 u} .
$$

Since $\sigma_{3} \mathbf{u}_{\alpha}(x)$ is a column of the $S$-matrix, it satisfies

$$
i \sigma_{3} \partial_{x} \mathbf{u}_{\alpha}(x)=M(x) \sigma_{3} \mathbf{u}_{\alpha}(x) .
$$

An analogous equation holds for $\mathbf{v}_{\alpha}(x)$. Taking the adjoint of Eq. (2.123) and remembering that $M^{\dagger}(x)=\sigma_{3} M(x) \sigma_{3}$, one gets

$$
i \partial_{x} \mathbf{u}_{\alpha}^{\dagger}(x)=-\mathbf{u}_{\alpha}^{\dagger}(x) M(x) .
$$

\section{Eilenberger equation}

Using Eqs. (2.123) and (2.124), it directly follows from the definition of the Eilenberger function that the partial derivative of $\tilde{g}^{R}(x)$ with respect to $x$ satisfies

$$
i \partial_{x} \tilde{g}(x)=[M(x), \tilde{g}(x)]
$$

where $[M(x), \tilde{g}(x)] \equiv M(x) \tilde{g}(x)-\tilde{g}(x) M(x)$ denotes the commutator of the matrices $M(x)$ and $g(x)$. In the literature of superconductivity, this equation is called the Eilenberger equation [21, 56]. While the Eilenberger equation was first derived using perturbation theory, a similar derivation to our derivation based on wave functions was given by Kos and Stone [52]. To derive a recurrence relation, we write $\tilde{g}(x)$ as

$$
\tilde{g}(x)=\sum_{n=0}^{\infty} \tilde{g}_{n}(x)
$$

where by definition $\tilde{g}_{n}(x)$ collects all terms involving $n$ gradients. It then follows

$$
i \partial_{x} \tilde{g}_{n}^{R}(x)=\left[M(x), \tilde{g}_{n+1}(x)\right] .
$$

Unfortunately, this equation can not be solved for $\tilde{g}_{n+1}(x)$. But since both $M(x)$ and $\tilde{g}_{n+1}(x)$ are traceless matrices, for a given $\tilde{g}_{n}(x)$ it is possible to determine $\tilde{g}_{n+1}(x)$ up to terms commuting with $M(x) 7$ To fix the term proportional to $M(x)$, we note that it

\footnotetext{
${ }^{7}$ Since $M(x)$ is a traceless matrix, we can write it as $M(x)=m(x) R^{\dagger}(x) \sigma_{3} R(x)$, where $m(x)$ is a scalar function and $R(x)$ is a (unitary) rotation matrix. Let us also expand $\tilde{g}_{n+1}$ as $\tilde{g}_{n+1}=R^{\dagger}(x)\left\{\alpha_{3}(x) \sigma_{3}+\right.$ $\left.\alpha_{-}(x) \sigma_{+}+\alpha_{+}(x) \sigma_{-}\right\} R(x)$, such that the first term on the right-hand side is proportional to $M(x)$. If we now use $\left[\sigma_{3}, \sigma_{ \pm}\right]= \pm 2 \sigma_{ \pm}$, we get $[M(x), \tilde{g}(x)]=2 m(x) R^{\dagger}(x)\left\{\alpha_{-}(x) \sigma_{+}-\alpha_{+}(x) \sigma_{-}\right\} R(x)$, which implies that only $\alpha_{+}(x)$ and $\alpha_{-}(x)$ can be evaluated from Eq. (2.127) while the term proportional to $M(x)$ remains undetermined.
} 
follows from Eq. (2.122) that $\tilde{g}(x)$ satisfies the constraint

$$
\tilde{g}^{2}(x)=-\frac{1}{4} \sigma_{0}
$$

Since $\tilde{g}(x)$ is a traceless matrix, it is possible to write $\tilde{g}(x)$ as

$$
\tilde{g}(x) \equiv \tilde{g}_{3}(x) \sigma_{3}+\tilde{g}_{-}(x) \sigma_{+}+\tilde{g}_{+}(x) \sigma_{-} .
$$

The Eilenberger equation 2.125 may now be rewritten as

$$
i \partial_{x}\left(\begin{array}{c}
\tilde{g}_{+}(x) \\
\tilde{g}_{3}(x) \\
\tilde{g}_{-}(x)
\end{array}\right)=\left(\begin{array}{ccc}
2 \tilde{\omega} & 2 \Delta^{*}(x) & 0 \\
-\Delta(x) & 0 & -\Delta^{*}(x) \\
0 & 2 \Delta(x) & -2 \tilde{\omega}
\end{array}\right)\left(\begin{array}{c}
\tilde{g}_{+}(x) \\
\tilde{g}_{3}(x) \\
\tilde{g}_{-}(x)
\end{array}\right) .
$$

This is a linear first-order differential equation, which we can cast into a pseudoSchrödinger equation.

\section{Pseudo-Schrödinger equation}

Defining $\vec{\psi}(x)$ and $\tilde{\psi}(x)$ as

$$
\vec{\psi}(x) \equiv\left(\begin{array}{c}
\sqrt{2} \tilde{g}_{+}(x) \\
-2 i \tilde{g}_{3}(x) \\
\sqrt{2} \tilde{g}_{-}(x)
\end{array}\right), \tilde{\psi}(x) \equiv\left(\begin{array}{c}
-\sqrt{2} \tilde{g}_{-}(x) \\
-2 i \tilde{g}_{3}(x) \\
-\sqrt{2} \tilde{g}_{+}(x)
\end{array}\right)
$$

the normalized local DOS $\rho(x, \omega) / \rho_{0}$ is simply given by the second component of $\vec{\psi}(x)$. The constraint (2.128) turns into

$$
\tilde{\psi}^{T}(x) \vec{\psi}(x)=1
$$

and Eq. (2.130) can be expressed as a pseudo-Schrödinger equation,

$$
-\partial_{x} \vec{\psi}(x)=H(x) \vec{\psi}(x)
$$

with the pseudo-Hamiltonian given by

$$
H(x)=\left(\begin{array}{ccc}
2 i \omega & \sqrt{2} \Delta^{*}(x) & 0 \\
\sqrt{2} \Delta(x) & 0 & \sqrt{2} \Delta^{*}(x) \\
0 & \sqrt{2} \Delta(x) & -2 i \omega
\end{array}\right)
$$

Note that $H(x)$ can also be written as

$$
H(x)=2 i \omega J_{3}+\Delta(x) J_{-}+\Delta^{*}(x) J_{+},
$$


where the $J_{i}$ are spin $J=1$ operators in the representation

$$
J_{3}=\left(\begin{array}{ccc}
1 & 0 & 0 \\
0 & 0 & 0 \\
0 & 0 & -1
\end{array}\right), J_{+}=\sqrt{2}\left(\begin{array}{ccc}
0 & 1 & 0 \\
0 & 0 & 1 \\
0 & 0 & 0
\end{array}\right), J_{-}=\sqrt{2}\left(\begin{array}{ccc}
0 & 0 & 0 \\
1 & 0 & 0 \\
0 & 1 & 0
\end{array}\right) .
$$

Formally, Eq. 2.133 looks like the imaginary-time Schrödinger equation for a $J=1$ quantum spin subject to an imaginary time-dependent magnetic field. Recall that the real part of the second component of the state $\vec{\psi}(x)$ can be identified with the local DOS. Because our pseudo-Schrödinger equation is linear, the gradient expansion of the local DOS can now be generated by a straightforward iterative calculation of the state $\vec{\psi}(x)$ in powers of gradients. This will be done in Appendix 



\section{Chapter 3}

\section{Exact results}

In this chapter, we analytically calculate the density of states (DOS) and the inverse localization length of the fluctuating gap model in the limits of very small and infinite correlation lengths $\xi$. While for $\xi=\infty$ we only have to average the DOS and the inverse localization length over an ensemble of systems with a space independent gap $\Delta(x)=\Delta_{0}$, in the white noise limit $\xi \rightarrow 0$, we can obtain the (integrated) DOS and the inverse localization length by deriving and solving an equation closely related to a Fokker-Planck equation.

\subsection{The white noise limit}

For small correlation lengths $\xi$, the disorder of the fluctuating gap model (FGM) may be approximated by Gaussian white noise. This $\xi \rightarrow 0$ limit is of special interest because in this case the disorder at different space points is uncorrelated which basically admits for an exact analytic solution of the model. Various methods may now be applied to find analytic results for the density of states (DOS). Ovchinnikov and Erikhman [71] were the first to solve the commensurate case for which the random backscattering potential $\Delta(x)$ is real. They showed that in the symmetric phase for which $\langle\Delta(x)\rangle=0$, the DOS has a Dyson singularity previously found by Dyson [19]. In the case of $\langle\Delta(x)\rangle \neq 0$ which models a phase below a phase transition, the DOS either exhibits a singularity or a pseudogap near the Fermi energy depending on the ratio of the disorder and the static gap. Using the technique of $S$-matrix summation, Golub and Chumakov [32] confirmed the results by Ovchinnikov and Erikhman and were also able to solve the incommensurate case. In the incommensurate case which is described by a complex backscattering potential, there is no singularity and the disorder can only lead to a filling up of the pseudogap. The incommensurate case was also considered by Abrikosov and Dorotheyev [3].

In recent years, the method of supersymmetry developed by Efetov [24] has been established as a powerful tool to describe disordered systems in the white noise limit. First, Hayn and John [40] re-derived the Ovchinnikov and Erikhman result for the 
DOS and were also able to give an analytic expression for the localization length. Later, Hayn and Fischbeck [29, 41] used the method of supersymmetry to generalize the known results for the integrated DOS to the case of three independent disorder parameters which describe forward, backward, and umklapp scattering. These solutions include both the commensurate and the incommensurate case as special cases. Finally, both the integrated DOS and the localization length were calculated by Hayn and Mertsching [42] in the most general case with a complex static gap parameter and three disorder parameters.

In this chapter we start from $i\langle\Gamma(\omega)\rangle=\omega+\left\langle\Delta^{*}(x) \exp [i \varphi(x)]\right\rangle$ and the equation of motion $\partial_{x} \varphi(x)=2[\omega-V(x)]+2|\Delta(x)| \cos [\varphi(x)-\vartheta(x)]$ which we derived in the previous chapter and follow the ideas of Lifshits, Gredeskul and Pastur [58] to show that the probability density for the distribution of the reduced phase $\varphi$ satisfies a continuity equation. The stationary probability flux of the continuity equation turns out to be equal to the integrated DOS. We then derive an equation closely related to a FokkerPlanck equation which allows to calculate the integrated DOS for the most general case exactly. Before considering the most general case we discuss the commensurate case, i.e. the Ovchinnikov and Erikhman limit which we can solve in analogy to Halperin's calculation of the integrated DOS of a particle with an effective mass in a white noise disorder potential [39]. The treatment of the general case is similar but more awkward than the Ovchinnikov and Erikhman limit because instead of a linear differential equation of second order one has to face a linear differential equation of fourth order. The equations to determine the integrated DOS and the localization length are, however, the same as those derived by Hayn and Mertsching using the method of supersymmetry [42] so that we recover their general results which also include the incommensurate case which we will discuss afterwards.

\subsubsection{Equality of the integrated density of states and the stationary probability flux}

As shown in the previous chapter, the averaged integrated DOS can be written in the thermodynamic limit as [see Eq. (2.96)]

$$
\mathcal{N}(\omega)=\left\langle F_{\omega}(\vec{\Delta}, \varphi)\right\rangle / 2 \pi
$$

where for notational brevity we use $\vec{\Delta}(x) \equiv(V(x), \Delta(x))$ and $F_{\omega}(\vec{\Delta}, \varphi)$ is a function linear in the disorder $V, \operatorname{Re} \Delta, \operatorname{Im} \Delta$,

$$
F_{\omega}(\vec{\Delta}, \varphi)=2(\omega-V)+2 \operatorname{Re} \Delta \cos \varphi+2 \operatorname{Im} \Delta \sin \varphi .
$$

The unreduced phase $\varphi(x, \omega)$ satisfies the equation of motion (2.89) which can also be written as

$$
\partial_{x} \varphi(x, \omega)=F_{\omega}(\vec{\Delta}(x), \varphi(x, \omega)) .
$$




\section{Continuity equation}

The space-dependent probability distribution can be defined as

$$
P_{\omega}(x, \varphi)=\left\langle\delta_{2 \pi}(\varphi-\varphi(x, \omega))\right\rangle,
$$

where $\delta_{2 \pi}(x) \equiv \sum_{m=-\infty}^{\infty} \delta(x-2 \pi m)$ is the $2 \pi$-periodic delta function. A continuity equation may be derived by partially differentiating the probability distribution $P_{\omega}(x, \varphi)$ with respect to $x$ :

$$
\partial_{x} P_{\omega}(x, \varphi)=-\partial_{\varphi}\left\langle\delta_{2 \pi}(\varphi-\varphi(x, \omega)) \partial_{x} \varphi(x, \omega)\right\rangle .
$$

Making use of Eq. (3.3), the continuity equation reads

$$
\partial_{x} P_{\omega}(x, \varphi)+\partial_{\varphi} J_{\omega}(x, \varphi)=0
$$

where the probability flux $J_{\omega}(x, \varphi)$ is given by

$$
J_{\omega}(x, \varphi)=\left\langle\delta_{2 \pi}(\varphi-\varphi(x, \omega)) F_{\omega}(\vec{\Delta}(x), \varphi(x, \omega))\right\rangle .
$$

Letting $x$ go to infinity, the probability distribution and the probability flux become stationary, i.e. independent of $x$. Due to the continuity equation (3.6), $J_{\omega}$ becomes also independent of $\varphi$. Integrating the stationary form of Eq. (3.7) with respect to $\varphi$ from 0 to $2 \pi$ therefore leads to $2 \pi J_{\omega}=\left\langle F_{\omega}(\vec{\Delta}, \varphi)\right\rangle$, so that together with Eq. (3.1) we find the remarkable relationship [58]

$$
\mathcal{N}(\omega)=J_{\omega}
$$

The integrated DOS, i.e. the number of states in the energy interval $(0, \omega]$ per unit length, is equal to the stationary probability flux. Note that this result is valid for any disorder potential and is not restricted to the white noise limit which we will consider in the following.

\subsubsection{White noise and the Fokker-Planck equation}

While for arbitrary finite correlation lengths $\xi$ it does not seem to be possible to find an exact analytic expression for the (integrated) DOS, the white noise limit $\xi \rightarrow 0$, $V_{\sigma}^{2 \xi} \rightarrow D_{V},\left(\operatorname{Re} \Delta_{\sigma}\right)^{2} \xi \rightarrow D_{R}$, and $\left(\operatorname{Im} \Delta_{\sigma}\right)^{2 \xi} \rightarrow D_{I}$ admits for an exact solution. This is due to the fact that in this case the disorder at different space points is uncorrelated.

In the white noise limit, the disorder is characterized by the following correlation functions:

$$
\begin{aligned}
\langle V(x)\rangle=0 \quad, \quad\left\langle V(x) V\left(x^{\prime}\right)\right\rangle=2 D_{V} \delta\left(x-x^{\prime}\right), \\
\langle\operatorname{Re} \Delta(x)\rangle=\operatorname{Re} \Delta_{0} \quad, \quad\left\langle\operatorname{Re} \tilde{\Delta}(x) \operatorname{Re} \tilde{\Delta}\left(x^{\prime}\right)\right\rangle=2 D_{R} \delta\left(x-x^{\prime}\right), \\
\langle\operatorname{Im} \Delta(x)\rangle=\operatorname{Im} \Delta_{0} \quad, \quad\left\langle\operatorname{Im} \tilde{\Delta}(x) \operatorname{Im} \tilde{\Delta}\left(x^{\prime}\right)\right\rangle=2 D_{I} \delta\left(x-x^{\prime}\right),
\end{aligned}
$$


where $\tilde{\Delta}(x) \equiv \Delta(x)-\Delta_{0}$. It is no loss of generality to assume that the first moment of the forward scattering potential $V(x)$ vanishes because a finite value would only lead to a renormalization of $\omega$. Since the probability distribution of the disorder is assumed to be Gaussian, higher correlation functions are simply given by Wick's theorem.

To cast the continuity equation into a Fokker-Planck equation, we make use of the Gaussian nature of the disorder, so that for a functional $f\{V(y)\}$ of the disorder $V(y)$ we have

$$
\langle V(x) f\{V(y)\}\rangle=\int d x^{\prime}\left\langle V(x) V\left(x^{\prime}\right)\right\rangle\left\langle\frac{\delta f\{V(y)\}}{\delta V\left(x^{\prime}\right)}\right\rangle,
$$

where in the last term we have to take the functional derivative of $f\{V(y)\}$ with respect to $V\left(x^{\prime}\right)$. Using Eq. (3.9), this simplifies to

$$
\langle V(x) f\{V(y)\}\rangle=2 D_{V}\left\langle\frac{\delta f\{V(y)\}}{\delta V(x)}\right\rangle
$$

Life becomes a little bit more subtle if we want to apply this relation to Eq. (3.7) because in this case, $f\{V(y)\}$ has to be replaced by the $2 \pi$-periodic delta-function $\delta_{2 \pi}(\varphi-\varphi(x, \omega))$ whose phase $\varphi(x, \omega)$ is a functional of the disorder involving the disorder at all space points $y \leq x$. Using the chain rule for the functional derivative we get

$$
\left\langle\delta_{2 \pi}(\varphi(x, \omega)-\varphi) V(x)\right\rangle=-2 D_{V} \partial_{\varphi}\left\langle\delta_{2 \pi}(\varphi(x, \omega)-\varphi) \frac{\delta \varphi(x, \omega)}{\delta V(x)}\right\rangle
$$

We now write $\varphi(x, \omega)$ as

$$
\varphi(x, \omega)-\varphi(0, \omega)=\int_{0}^{x} d x^{\prime} \partial_{x^{\prime}} \varphi\left(x^{\prime}, \omega\right)=\int_{0}^{x} d x^{\prime} F_{\omega}\left(\vec{\Delta}\left(x^{\prime}\right), \varphi\left(x^{\prime}, \omega\right)\right)
$$

and find

$$
\frac{\delta \varphi(x, \omega)}{\delta V\left(x^{\prime}\right)}=-2 \theta\left(x-x^{\prime}\right)
$$

Since in our case $x=x^{\prime}, \theta(0)$ needs to be defined carefully (see also Itzykson and Drouffe [43]). Recalling that we have introduced the delta function $\delta(x)$ as the limit $\xi \rightarrow 0$ of a symmetric function of $x$, we see that to maintain this symmetry, we have to define $\theta(0)=1 / 2$. It therefore follows with $P_{\omega}(x, \varphi)=\left\langle\delta_{2 \pi}(\varphi-\varphi(x, \omega))\right\rangle$ that

$$
\left\langle\delta_{2 \pi}(\varphi-\varphi(x, \omega)) V(x)\right\rangle=2 D_{V} \partial_{\varphi} P_{\omega}(x, \varphi) .
$$

Similarly, we can show that

$$
\begin{aligned}
& \left\langle\delta_{2 \pi}(\varphi-\varphi(x, \omega)) \operatorname{Re} \tilde{\Delta}(x)\right\rangle=-2 D_{R} \partial_{\varphi}\left(\cos \varphi P_{\omega}(x, \varphi)\right), \\
& \left\langle\delta_{2 \pi}(\varphi-\varphi(x, \omega)) \operatorname{Im} \tilde{\Delta}(x)\right\rangle=-2 D_{I} \partial_{\varphi}\left(\sin \varphi P_{\omega}(x, \varphi)\right) .
\end{aligned}
$$


Making use of these relations, the probability flux $J_{\omega}(x, \varphi)$ is given by

$$
\begin{gathered}
J_{\omega}(x, \varphi)=2\left[\omega+\operatorname{Re} \Delta_{0} \cos \varphi+\operatorname{Im} \Delta_{0} \sin \varphi\right] P_{\omega}(x, \varphi) \\
-4\left[D_{V} \partial_{\varphi} P_{\omega}(x, \varphi)+D_{R} \cos \varphi \partial_{\varphi}\left(\cos \varphi P_{\omega}(x, \varphi)\right)\right. \\
\left.+D_{I} \sin \varphi \partial_{\varphi}\left(\sin \varphi P_{\omega}(x, \varphi)\right)\right] .
\end{gathered}
$$

The probability flux can also be written as

$$
J_{\omega}(x, \varphi)=A_{\omega}(\varphi) P_{\omega}(x, \varphi)-\frac{1}{2} \partial_{\varphi}\left[B(\varphi) P_{\omega}(x, \varphi)\right]
$$

where

$$
\begin{aligned}
A_{\omega}(\varphi) & =2\left[\omega+\operatorname{Re} \Delta_{0} \cos \varphi+\operatorname{Im} \Delta_{0} \sin \varphi\right]-4\left(D_{R}-D_{I}\right) \cos \varphi \sin \varphi \\
B(\varphi) & =8\left[D_{V}+D_{R} \cos ^{2} \varphi+D_{I} \sin ^{2} \varphi\right] .
\end{aligned}
$$

Note that the forward scattering disorder $D_{V}$ only leads to a renormalization of $D_{R}$ and $D_{I}$. Let us therefore define $\tilde{D}_{R} \equiv D_{R}+D_{V}$ and $\tilde{D}_{I} \equiv D_{I}+D_{V}$.

Eq. (3.21) together with the continuity equation explicitly shows that the probability distribution satisfies the following one-dimensional Fokker-Planck equation [34, 46]:

$$
\partial_{x} P_{\omega}(x, \varphi)=-\partial_{\varphi}\left[A_{\omega}(\varphi) P_{\omega}(x, \varphi)\right]+\frac{1}{2} \partial_{\varphi}^{2}\left[B(\varphi) P_{\omega}(x, \varphi)\right]
$$

The first term on the right-hand side is often called the transport, drift or convection term. The second term is known as the diffusion or fluctuation term.

To find an analytic expression for the integrated DOS, we use the fact that, as shown above, $\mathcal{N}(\omega)$ is equal to the stationary probability flux. A good starting point to calculate the integrated DOS is therefore the stationary form of Eq. (3.21), which is nothing but the integrated stationary Fokker-Planck equation with the constant of integration being the stationary probability flux that is equal to the integrated DOS $\mathcal{N}(\omega)$,

$$
\mathcal{N}(\omega)=A_{\omega}(\varphi) P_{\omega}(\varphi)-\frac{1}{2} \partial_{\varphi}\left[B(\varphi) P_{\omega}(\varphi)\right] .
$$

In principle, one could first find a solution for $\left[B(\varphi) P_{\omega}(\varphi)\right]$ to this first-order differential equation subject to the boundary condition $\left[B(2 \pi) P_{\omega}(2 \pi)\right]=\left[B(0) P_{\omega}(0)\right]$ with $\mathcal{N}(\omega)$ as a parameter and could then use the normalization condition $\int_{0}^{2 \pi} d \varphi P_{\omega}(\varphi)=1$ to determine $\mathcal{N}(\omega)$. While this procedure works quite well in the incommensurate case with $D_{R}=D_{I}$ and after a variable transformation also in the commensurate case (without forward scattering) for which $D_{I}=D_{V}=0$, the most general case considered here seems to defy such a treatment. Here, we therefore present an alternative method which allows to recover all known results in the white noise limit. 


\subsubsection{The density of states in the white noise limit}

To find the (integrated) DOS for arbitrary parameters $D_{R}, D_{I}, D_{V}$ and complex $\Delta_{0}$, we first make the variable transformation

$$
z=\tan \left(\frac{\varphi}{2}-\frac{\pi}{4}\right)
$$

The trigonometric functions $\sin \varphi$ and $\cos \varphi$ can now be expressed in terms of $z$ : $\sin \varphi=\frac{1-z^{2}}{1+z^{2}}$, and $\cos \varphi=-\frac{2 z}{1+z^{2}}$. Since $\frac{d z}{d \varphi}=\frac{1}{2}\left(1+z^{2}\right)$, we find $\partial_{\varphi}=\frac{1}{2}\left(1+z^{2}\right) \partial_{z}$, and the probability distribution $P(\varphi)$ has to be replaced by $\frac{1}{2}\left(1+z^{2}\right) P(z)$. Using these relations, Eq. (3.25) turns into

$$
\begin{gathered}
\mathcal{N}(\omega)=\left[\left(\omega+\operatorname{Im} \Delta_{0}\right)-2\left(\operatorname{Re} \Delta_{0}-\left(2 \tilde{D}_{R}-\tilde{D}_{I}\right)\right) z+\left(\omega-\operatorname{Im} \Delta_{0}\right) z^{2}\right. \\
\left.+2 \tilde{D}_{I} z^{3}\right] P(z)-\tilde{D}_{I} \partial_{z}\left(\left[1+2 \tilde{D}_{I}^{-1}\left(2 \tilde{D}_{R}-\tilde{D}_{I}\right) z^{2}+z^{4}\right] P(z)\right) .
\end{gathered}
$$

Taking the Fourier transform of this equation leads to

$$
\begin{aligned}
2 \pi \mathcal{N}(\omega) \delta(k) & =\left(\omega+\operatorname{Im} \Delta_{0}\right) \tilde{P}(k)-2 i\left(\operatorname{Re} \Delta_{0}-\left(2 \tilde{D}_{R}-\tilde{D}_{I}\right)\right) \tilde{P}^{\prime}(k) \\
& -\left(\omega-\operatorname{Im} \Delta_{0}\right) \tilde{P}^{\prime \prime}(k)-2 i \tilde{D}_{I} \tilde{P}^{\prime \prime \prime}(k) \\
& -i \tilde{D}_{I} k\left[\tilde{P}(k)-2 \tilde{D}_{I}^{-1}\left(2 \tilde{D}_{R}-\tilde{D}_{I}\right) \tilde{P}^{\prime \prime}(k)+\tilde{P}^{\prime \prime \prime \prime}(k)\right]
\end{aligned}
$$

where

$$
\tilde{P}(k)=\int_{-\infty}^{\infty} e^{-i k z} P(z) d z
$$

is the Fourier transform of $P(z)$ which is also known as the characteristic function [28]. Normalization of the probability distribution $P(z)$ implies $\tilde{P}(k=0)=1$. Assuming higher derivatives of $P(z)$ to be integrable, the other boundary conditions are given by demanding that $\tilde{P}(k) \rightarrow 0$ sufficiently rapidly as $|k| \rightarrow \infty$.

\subsubsection{The commensurate case without forward scattering}

Before we proceed with the most general case, let us first consider the Ovchinnikov and Erikhman limit, i.e. the commensurate case without forward scattering. In this case, $\Delta_{0}$ is real and may be assumed to be positive, $D_{I}=D_{V}=0$ and we set $D \equiv D_{R}$. Eq. (3.28) reduces to a second order differential equation with the boundary conditions $\tilde{P}(0)=1$ and $\tilde{P}(k) \rightarrow 0$ as $|k| \rightarrow \infty$ :

$$
2 \pi \mathcal{N}(\omega) \delta(k)=\omega \tilde{P}(k)+4 i D\left(1-\frac{\Delta_{0}}{2 D}\right) \tilde{P}^{\prime}(k)+4 i D\left(k+\frac{i \omega}{4 D}\right) \tilde{P}^{\prime \prime}(k) .
$$

Integrating this equation from $-\varepsilon$ to $+\varepsilon$ with $\varepsilon \rightarrow 0^{+}$leads to

$$
2 \pi \mathcal{N}(\omega)=-\omega\left[\tilde{P}^{\prime}\left(0^{+}\right)-\tilde{P}^{\prime}\left(0^{-}\right)\right] .
$$


Since $P(z)$ is real, $\tilde{P}(-k)=\tilde{P}^{*}(k)$, and $\tilde{P}^{\prime}(-k)=-\tilde{P}^{\prime *}(k)$, which implies

$$
\mathcal{N}(\omega)=-\frac{\omega}{\pi} \operatorname{Re} \tilde{P}^{\prime}\left(0^{+}\right) .
$$

$\tilde{P}^{\prime}\left(0^{+}\right)$can be determined by first finding a solution to the differential equation (3.30) for $k>0$ which vanishes as $k$ approaches infinity and then normalizing this solution such that $\tilde{P}(0)=1$. Because Eq. (3.30) is homogeneous for $k>0$, it therefore follows that if $g(k)$ is any solution to the homogeneous differential equation which obeys $g(k) \rightarrow 0$ as $k \rightarrow \infty$, then $\mathcal{N}(\omega)=-\frac{\omega}{\pi} \operatorname{Re}\left[g^{\prime}(0) / g(0)\right]$. We introduce $y(t) \equiv g(k)$ with $t=-i(k+i \omega / 4 D)$. The integrated DOS is now given by

$$
\mathcal{N}(\omega)=\frac{\omega}{\pi} \operatorname{Im}\left(\frac{y^{\prime}\left(\frac{\omega}{4 D}\right)}{y\left(\frac{\omega}{4 D}\right)}\right),
$$

where $y(t)$ has to satisfy the differential equation

$$
t y^{\prime \prime}(t)+\left(1-\frac{\Delta_{0}}{2 D}\right) y^{\prime}(t)+\frac{\omega}{4 D} y(t)=0
$$

with the only restriction that $y(t)$ should approach zero as $t$ goes to $i \infty$. The general solution of this differential equation can be expressed in terms of a linear combination of Bessel functions of the first and second kind, $J_{v}(x)$ and $N_{v}(x)$ (Abramowitz and Segun $(A \& S)[4])$. The only solution which satisfies the boundary condition involves the Hankel function of the first kind, $H_{v}^{(1)}(x)=J_{v}(x)+i N_{v}(x)$. Introducing

$$
v=\frac{\Delta_{0}}{2 D}
$$

we find $y(t)=(v / 2)^{t} H_{\mathrm{v}}^{(1)}\left((\omega t / D)^{1 / 2}\right)$. Because the prefactor is real and we only need the imaginary part of the quotient $y^{\prime}(\omega / 4 D) / y(\omega / 4 D)$, Eq. (3.33) turns into

$$
\mathcal{N}(\omega)=\frac{\omega}{\pi} \operatorname{Im}\left(\frac{\left[H_{\mathrm{v}}^{(1)}\left(\frac{\omega}{2 D}\right)\right]^{\prime}}{H_{\mathrm{v}}^{(1)}\left(\frac{\omega}{2 D}\right)}\right) .
$$

Note that this equation is valid for arbitrary $\omega$ and is even analytic in the upper half plane. In the following discussion of the Ovchinnikov and Erikhman limit, we will again restrict ourselves to $\omega>0$. It follows

$$
\mathcal{N}(\omega)=\frac{\omega}{\pi} \frac{J_{v}\left(\frac{\omega}{2 D}\right) N_{v}^{\prime}\left(\frac{\omega}{2 D}\right)-J_{v}^{\prime}\left(\frac{\omega}{2 D}\right) N_{v}\left(\frac{\omega}{2 D}\right)}{J_{v}^{2}\left(\frac{\omega}{2 D}\right)+N_{v}^{2}\left(\frac{\omega}{2 D}\right)} .
$$

The numerator can be simplified by using the Wronski relation [A\&S, Eq. (9.1.16)]

$$
J_{v}(x) N_{v}^{\prime}(x)-J_{v}^{\prime}(x) N_{v}(x)=\frac{2}{\pi x},
$$


so that as our final expression for the integrated DOS we are left with

$$
\mathcal{N}(\omega)=\frac{4 D}{\pi^{2}\left[J_{v}^{2}\left(\frac{\omega}{2 D}\right)+N_{v}^{2}\left(\frac{\omega}{2 D}\right)\right]}
$$

This result was first obtained by Ovchinnikov and Erikhman [71] in a more complicated manner. Differentiating Eq. (3.39) with respect to $\omega$, we find

$$
\rho(\omega)=-\frac{4\left[J_{v}\left(\frac{\omega}{2 D}\right) J_{v}^{\prime}\left(\frac{\omega}{2 D}\right)+N_{v}\left(\frac{\omega}{2 D}\right) N_{v}^{\prime}\left(\frac{\omega}{2 D}\right)\right]}{\pi^{2}\left[J_{v}^{2}\left(\frac{\omega}{2 D}\right)+N_{v}^{2}\left(\frac{\omega}{2 D}\right)\right]^{2}} .
$$

Finally, we can use the relations [A\&S, Eq. (9.1.27)]

$$
2 J_{v}^{\prime}(x)=J_{v-1}(x)+J_{v+1}(x) \quad \text { and } \quad 2 N_{v}^{\prime}(x)=N_{v-1}(x)+N_{v+1}(x)
$$

to express the derivatives of the Bessel functions through Bessel functions. Defining $u \equiv \omega / 2 D$, we get

$$
\rho(\omega)=\frac{2\left[J_{v}(u)\left[J_{v+1}(u)-J_{v-1}(u)\right]+N_{v}(u)\left[N_{v+1}(u)-N_{v-1}(u)\right]\right]}{\pi^{2}\left[J_{v}^{2}(u)+N_{v}^{2}(u)\right]^{2}} .
$$

For $\Delta_{0}=0$ which implies $v=0, D$ is the only characteristic energy scale, and it is useful to measure all energies in terms of $D$. Figure 3.1 shows the DOS $\rho(\omega)$ plotted versus $\omega / D$. The DOS clearly exhibits a singularity near $\omega=0$ whose asymptotic behavior can be found by noting that $J_{0}(0)$ is finite while $N_{0}(x)$ diverges logarithmically for small $x, N_{0}(x) \sim 2 / \pi \ln x$ (A\&S, Eq. 9.1.8). It follows 1

$$
\mathcal{N}(\omega) \sim \frac{D}{\ln ^{2}(\omega / 2 D)}
$$

such that the asymptotics of the DOS for $\Delta_{0}=0$ is given by

$$
\rho(\omega) \sim-\frac{1}{(\omega / 2 D) \ln ^{3}(\omega / 2 D)} .
$$

Recall that to generalize this result towards arbitrary frequencies $\omega$, we have to replace $\omega$ by $|\omega|$. The singularity described by Eq. (3.44) is called a Dyson singularity and was first found by Dyson in a different model [19] involving also off-diagonal disorder. Outside this singularity, the DOS is almost equal to the DOS of the disorder-free model, taking its minima $\rho\left(\omega^{*}\right)=0.9636 \rho_{0}$ at $\omega^{*}= \pm 1.2514 D$.

If $\Delta_{0} \neq 0, \Delta_{0}$ is another characteristic energy scale. $v \equiv \Delta_{0} / 2 D$ then basically gives the ratio of the two relevant energy scales $\Delta_{0}$ and $D$. The DOS plotted against $\omega / \Delta_{0}$ for different values of the parameter $v$ is shown at the top of Fig. 3.2. While the limit

\footnotetext{
${ }^{1}$ Note that the argument of the logarithm in the asymptotic form given by Ovchinnikov and Erikhman deviates by a factor $1 / 2$ from our result. Nevertheless both expressions lead to the same asymptotic behavior. To take into account next to leading terms one has to use $N_{0}(x)=2 / \pi \ln (a x)+O(x)$, where $a=e^{\gamma} / 2$ which follows from A\&S, Eq. (9.1.89). Here, $\gamma \approx 0.5772$ is Euler's constant which leads to $a \approx 0.8905$. This value is closer to our choice $a=1$ than to Ovchinnikovt's and Erikhman's choice $a=1 / 2$.
} 


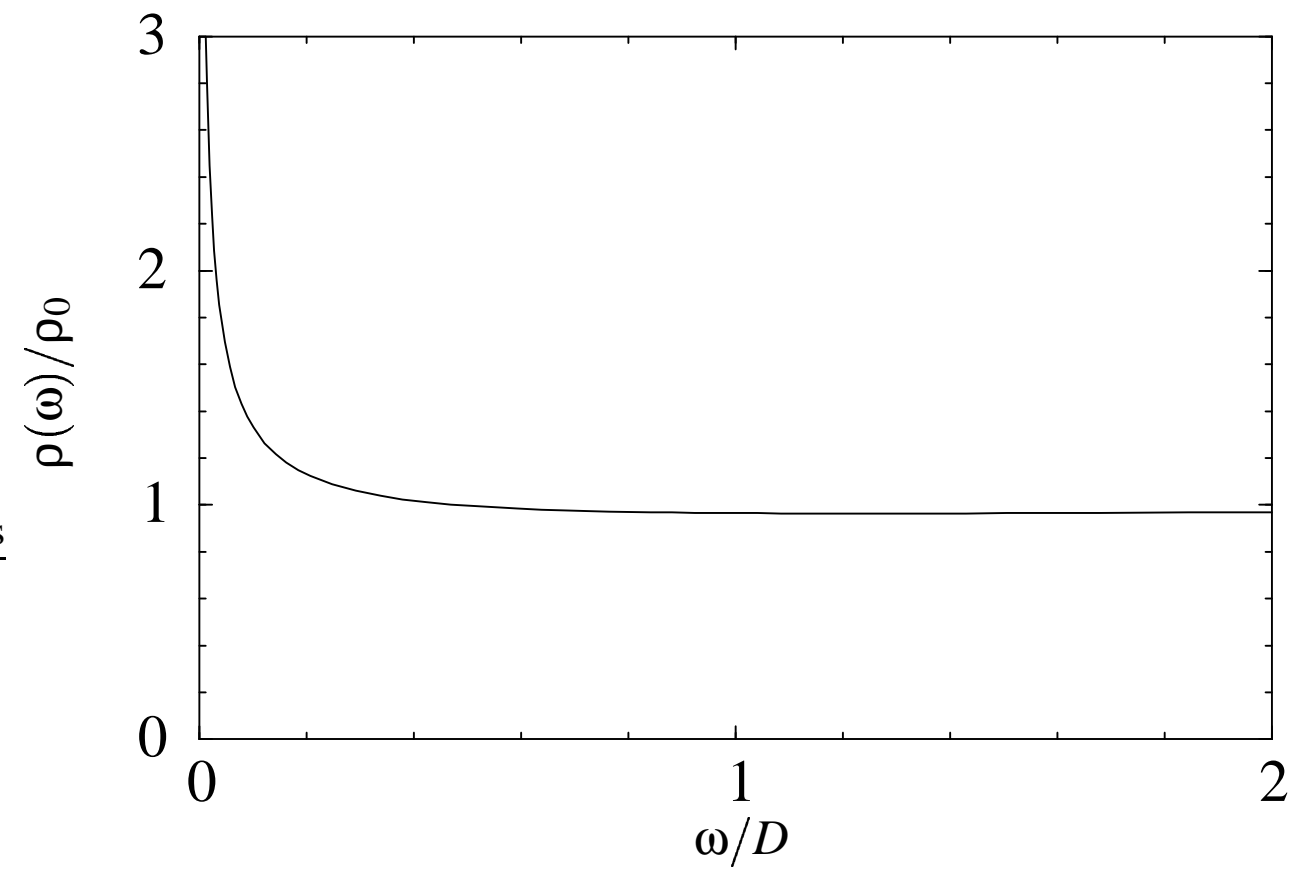

Figure 3.1: Dyson singularity in the DOS $\rho(\omega)$ for $\Delta_{0}=0$.

$v \rightarrow 0$, for which one should measure $\omega$ in units of $D$, was already considered above, in this figure we see that the singularity only survives for $v<1 / 2$. For $v=1 / 2$, the DOS is constant and for $v>1 / 2$ the effects of the constant gap $\Delta_{0}$ dominate those due to the disorder and a pseudogap emerges.

The algebraic dependence of the DOS at small $\omega$ on the parameter $v$ can be found by using again an asymptotic expansion of the Bessel functions. If $v>0$ is fixed and $x \rightarrow 0$, the Bessel function $J_{v}(x)$ stays finite and $N_{v}(x) \sim-(1 / \pi) \Gamma(v)(x / 2)^{-v}[\mathrm{~A} \& \mathrm{~S}$, Eq. (9.1.9)]. It follows

$$
\mathcal{N}(\omega) \sim \frac{4 D}{\Gamma^{2}(v)}\left(\frac{\omega}{4 D}\right)^{2 v}
$$

so that

$$
\rho(\omega) \sim \frac{2 v}{\Gamma^{2}(v)}\left(\frac{\omega}{4 D}\right)^{2 v-1} .
$$

We now see that for $v<1 / 2$ the DOS in fact diverges algebraically as $\omega$ approaches zero because in this case the exponent is negative. Although the algebraic divergence differs from the divergence found in Dyson's model, we will nevertheless refer to this singularity as a Dyson singularity. For $v>1 / 2$, however, the exponent is positive and the DOS vanishes algebraically.

For large $v$ the disorder becomes irrelevant, $\mathcal{N}(\omega)=\rho_{0} \theta\left(\omega^{2}-\Delta_{0}^{2}\right)\left(\omega^{2}-\Delta_{0}^{2}\right)^{1 / 2}$, 

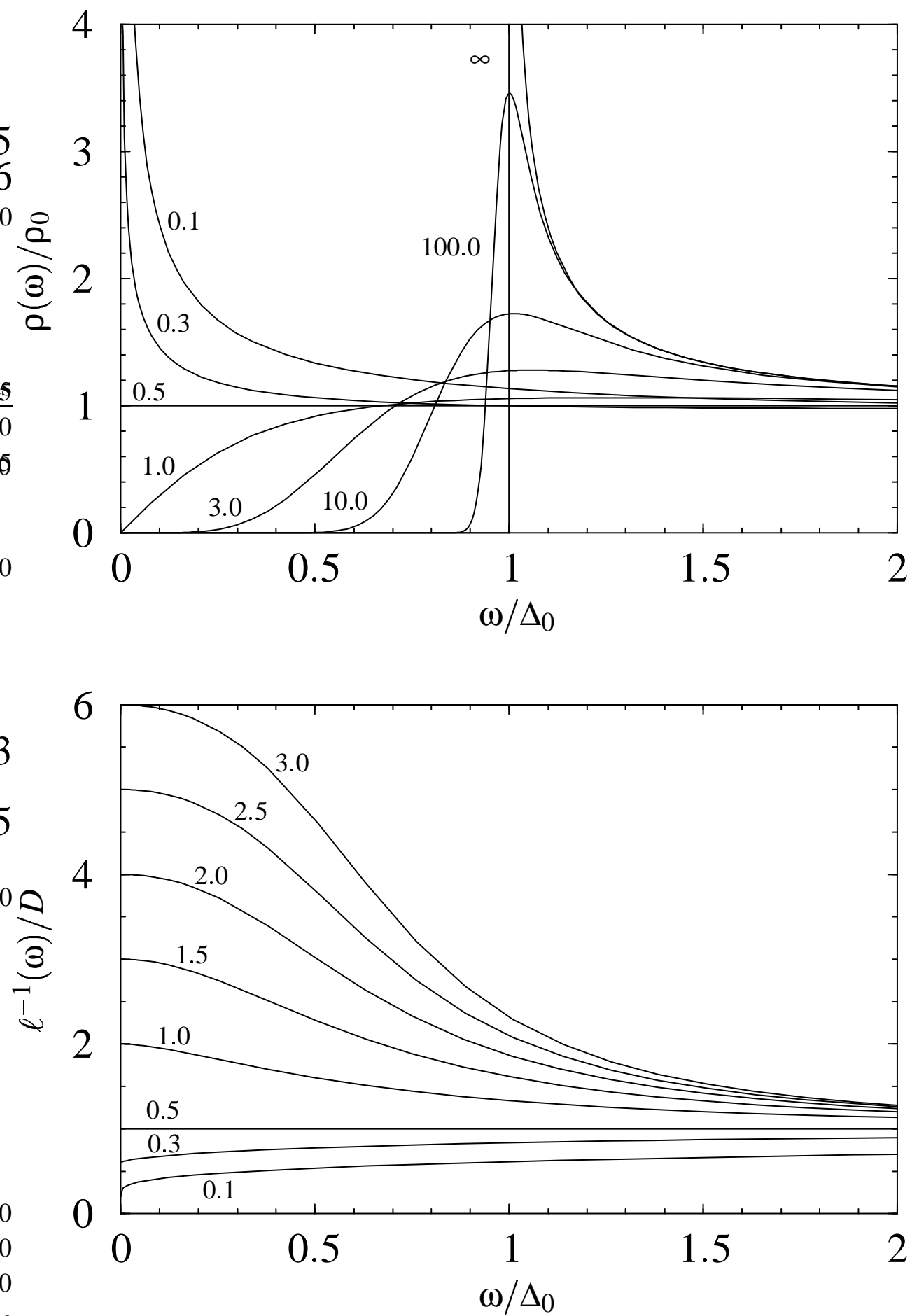

Figure 3.2: The DOS $\rho(\omega)$ and the inverse localization length $\ell^{-1}(\omega)$ in the white noise limit for the commensurate case plotted versus $\omega / \Delta_{0}$ for $v=\Delta_{0} / 2 D=$ $0.1,0.3,0.5,1.0,3.0,10.0,100.0, \infty$ or $v=\Delta_{0} / 2 D=0.1,0.3,0.5,1.0,1.5,2.0,2.5,3.0$, respectively. 
and the DOS reduces to the mean-field result

$$
\rho(\omega)=\left\{\begin{array}{cc}
0 & , \omega<\Delta_{0} \\
\rho_{0} \frac{\omega}{\left(\omega^{2}-\Delta_{0}^{2}\right)^{1 / 2}} & , \omega>\Delta_{0}
\end{array}\right.
$$

It is also easy to see why the DOS is constant for $v=1 / 2$. In this case the Bessel functions are of half odd integral order and can be expressed in terms of $\sin x, \cos x$, and powers of $x$. Using the well-known addition theorem $\cos ^{2} x+\sin ^{2} x=1$, one finds $\mathcal{N}(\omega)=\omega / \pi$. The DOS is then given by $\rho(\omega)=\rho_{0}$ where $\rho_{0}=1 / \pi$ : The effects of the disorder are exactly canceled by those of the static gap.

\section{Localization length}

Since $\Gamma(\omega) \equiv \ell^{-1}(\omega)-i \pi \mathcal{N}(\omega)$ is an analytic function in the upper half plane, we can also easily find an analytic expression for the localization length $\ell^{-1}(\omega)$. It follows from Eq. 3.36 that up to a constant

$$
\Gamma(\omega)=-\omega \frac{\left[H_{v}^{(1)}\left(\frac{\omega}{2 D}\right)\right]^{\prime}}{H_{v}^{(1)}\left(\frac{\omega}{2 D}\right)}
$$

The inverse localization length is now given by

$$
\ell^{-1}(\omega)=\operatorname{Re} \Gamma(\omega)=-\omega \frac{J_{v}\left(\frac{\omega}{2 D}\right) J_{v}^{\prime}\left(\frac{\omega}{2 D}\right)+N_{v}\left(\frac{\omega}{2 D}\right) N_{v}^{\prime}\left(\frac{\omega}{2 D}\right)}{J_{v}^{2}\left(\frac{\omega}{2 D}\right)+N_{v}^{2}\left(\frac{\omega}{2 D}\right)} .
$$

Comparing the right hand side of this equation with Eqs. (3.39) and (3.40), we find

$$
\ell^{-1}(\omega)=D \frac{\omega \rho(\omega)}{\mathcal{N}(\omega)}
$$

This equation is exact and can already be found on page 155 in the book by Lifshits, Gredeskul and Pastur [58].

If $\Delta_{0}=0$, it follows from Eqs. (3.43) and (3.44) that $\ell^{-1}(\omega)$ vanishes logarithmically as $\omega$ approaches zero,

$$
\ell^{-1}(\omega) \sim-\frac{2 D}{\ln (\omega / 2 D)}
$$

Using Eqs. (3.45) and (3.46), we get for arbitrary $\Delta_{0}$

$$
\ell^{-1}(0)=\Delta_{0}
$$


which, as can be seen from Eq. (3.51) is also true for $\Delta_{0}=0$. Eq. (3.52) agrees with Eq. (2.121), so that $\Gamma(\omega)$ involves no extra constant. For large frequencies, $\rho(\omega) \rightarrow \rho_{0}$ and $\mathcal{N}(\omega) \rightarrow \rho_{0} \omega$, such that

$$
\ell^{-1}(\omega) \rightarrow D
$$

A plot of the inverse localization length $\ell^{-1}(\omega)$ for various values of $v=\Delta_{0} / 2 D$ is given at the bottom of Fig. (3.2). Note that here we measure $\ell^{-1}(\omega)$ in terms of $D$ but $\omega$ in terms of $\Delta_{0}$.

\subsubsection{Solving the general case with arbitrary parameters $D_{R}, D_{I}$, $D_{V}$ and $\Delta_{0}$}

While in the commensurate case we only had to solve a differential equation of second order, for $D_{I} \neq 0$, Eq. (3.28) is a differential equation of fourth order and more difficult to solve. Without loss of generality we may assume that $\tilde{D}_{R}>\tilde{D}_{I}$ (later we can also take the limit $\tilde{D}_{R} \rightarrow \tilde{D}_{I}$ ). Integrating Eq. (3.28) from $-\varepsilon$ to $+\varepsilon$ with $\varepsilon \rightarrow 0^{+}$we can proceed as before and express the integrated DOS in terms of $\tilde{P}(k)$ and derivatives thereof evaluated at $\pm 0^{+}$. Integrating the terms linear in $k$ by parts and using again the fact that $\tilde{P}^{(n)}(-k)=(-1)^{n} \tilde{P}^{(n) *}(k)$, we find

$$
\mathcal{N}(\omega)=\frac{1}{\pi} \operatorname{Im}\left[-i\left(\omega-\operatorname{Im} \Delta_{0}\right) \tilde{P}^{\prime}\left(0^{+}\right)+D_{I} \tilde{P}^{\prime \prime}\left(0^{+}\right)\right] .
$$

So, if $y(k)$ is any solution to the homogeneous differential equation

$$
\begin{aligned}
& \left(\omega+\operatorname{Im} \Delta_{0}\right) y(k)-2 i\left(\operatorname{Re} \Delta_{0}-\left(2 \tilde{D}_{R}-\tilde{D}_{I}\right)\right) y^{\prime}(k)-\left(\omega-\operatorname{Im} \Delta_{0}\right) y^{\prime \prime}(k) \\
& \quad-2 i \tilde{D}_{I} y^{\prime \prime \prime}(k)-i \tilde{D}_{I} k\left[y(k)-2 \tilde{D}_{I}^{-1}\left(2 \tilde{D}_{R}-\tilde{D}_{I}\right) y^{\prime \prime}(k)+y^{\prime \prime \prime \prime}(k)\right]=0,
\end{aligned}
$$

which vanishes for $k \rightarrow \infty$ sufficiently rapidly, then the integrated DOS is given by

$$
\mathcal{N}(\omega)=\frac{1}{\pi} \operatorname{Im}\left[-i\left(\omega-\operatorname{Im} \Delta_{0}\right) \frac{y^{\prime}(0)}{y(0)}+D_{I} \frac{y^{\prime \prime}(0)}{y(0)}\right] .
$$

Again, since $\Gamma(\omega) \equiv \ell^{-1}(\omega)-i \pi \mathcal{N}(\omega)$ is an analytic function in the upper half plane, up to a constant we have

$$
\Gamma(\omega)=i\left(\omega-\operatorname{Im} \Delta_{0}\right) \frac{y^{\prime}(0)}{y(0)}-\tilde{D}_{I} \frac{y^{\prime \prime}(0)}{y(0)} .
$$

Using the method of supersymmetry invented by Efetov [24], Hayn and Mertsching [42] derived the set of equations (3.55) and (3.57) by different means. Using the method of Laplace transforms they found an exact expression with the constant of

\footnotetext{
${ }^{2}$ Hayn and Mertsching use a slightly different notation but apart from this and some irrelevant different signs their expressions are equal to ours.
} 
integration chosen such that one obtains the correct asymptotic behavior for large frequencies determined in the Born approximation, $\Gamma(\omega)=D-i \omega=D_{R}+D_{I}-i \omega$. Here, instead of presenting a lengthy derivation, we will only cite the exact result found in [42]:

$$
\Gamma(\omega)=2 D_{I}+4 \tilde{D}_{R}\left[z(1-z) \frac{F^{\prime}(z)}{F(z)}+z \delta_{R}-i(1-z) \varepsilon\right] .
$$

In this equation, $F(z)$ is the hypergeometric function

$$
F(z)=F\left(\frac{1}{2}-i \varepsilon+i \delta_{I}-\delta_{R}, \frac{1}{2}-i \varepsilon-i \delta_{I}-\delta_{R}, 1-2 i \varepsilon ; z\right)
$$

with the parameters $\delta_{R}, \delta_{I}, \varepsilon$, and $z$ (in our notation) given by

$$
\begin{gathered}
\delta_{R}=\frac{\operatorname{Re} \Delta_{0}}{4\left(\tilde{D}_{R}\left(\tilde{D}_{R}-\tilde{D}_{I}\right)\right)^{1 / 2}} \quad, \quad \delta_{I}=\frac{\operatorname{Im} \Delta_{0}}{4\left(\tilde{D}_{I}\left(\tilde{D}_{R}-\tilde{D}_{I}\right)\right)^{1 / 2}}, \\
\varepsilon=\frac{\omega}{4\left(\tilde{D}_{R} \tilde{D}_{I}\right)^{1 / 2}} \quad, \quad z=\frac{\tilde{D}_{R}-\tilde{D}_{I}}{\tilde{D}_{R}} .
\end{gathered}
$$

Recall that we incorporated the parameter of the forward scattering disorder, $D_{V}$, into $D_{R}$ and $D_{I}$ by defining $\tilde{D}_{R} \equiv D_{R}+D_{V}$ and $\tilde{D}_{I} \equiv D_{I}+D_{V}$. Only the additive constant $2 D_{I}$ in Eq. (3.58) does not get renormalized by $D_{V}$. This is due to the fact that for large frequencies $\Gamma(\omega) \sim D_{R}+D_{I}-i \omega$, independent of $D_{V}$. As one would expect, Eq. (3.58) is invariant under $\operatorname{Re} \Delta_{0} \rightarrow-\operatorname{Re} \Delta_{0}$ and $\operatorname{Im} \Delta_{0} \rightarrow-\operatorname{Im} \Delta_{0}$. This can be seen by noting that $F(a, b ; c ; z)=(1-z)^{c-a-b} F(c-a, c-b ; c ; z)$ and $F(a, b ; c ; z)=F(b, a ; c ; z)$.

The imaginary part of Eq. (3.58) determines the integrated DOS and can be simplified as follows: First of all, we write

$$
\mathcal{N}(\omega)=-\rho_{0} \operatorname{Im} \Gamma(\omega) \equiv \rho_{0} \frac{W}{|F|^{2}},
$$

where

$$
W(z)=-4 \tilde{D}_{R}(1-z)\left[z \operatorname{Im}\left(F^{\prime}(z) F^{*}(z)\right)-\varepsilon|F(z)|^{2}\right] .
$$

Differentiating Eq. (3.63) with respect to $z$, we see that $W(z)$ satisfies the Wronski relation

$$
\frac{d}{d z} W(z)=-\frac{2 \delta_{R}}{1-z} W(z)
$$

This differential equation can easily be integrated to give

$$
W(z)=W(0)(1-z)^{2 \delta_{R}}
$$

Since $F(0)=1$, it directly follows from Eq. (3.63) that $W(0)=4 \tilde{D}_{R} \varepsilon=\left(\tilde{D}_{R} / \tilde{D}_{I}\right)^{1 / 2} \omega$, such that

$$
W=\left(\frac{\tilde{D}_{R}}{\tilde{D}_{I}}\right)^{1 / 2-2 \delta_{R}} \omega
$$


The integrated DOS can therefore be written as

$$
\mathcal{N}(\omega)=\rho_{0}\left(\frac{\tilde{D}_{R}}{\tilde{D}_{I}}\right)^{1 / 2-2 \delta_{R}} \frac{\omega}{|F|^{2}} .
$$

Taking the confluent limit $D_{I} \rightarrow 0$ of Eq. (3.58), one can recover Eq. (3.48) which describes the commensurate case without forward scattering. Turning on the forward scattering disorder $D_{V}$ in the general expression gradually removes the possible singularity in the DOS. Below, we are only going to discuss the incommensurate case. Other special cases can be found in [29, 40, 41, 42, 65].

\subsubsection{The incommensurate case}

In the incommensurate case, $D_{R}=D_{I} \equiv D / 2$, and the forward scattering potential $D_{V}$ only leads to a renormalization of $D$. If we introduce $\tilde{D} \equiv D+D_{V}=\tilde{D}_{R} / 2+\tilde{D}_{I} / 2$, we can write $\Gamma(\omega)$ as

$$
\Gamma(\omega)=D-i \omega+2 \tilde{D} \frac{z F^{\prime}}{F} .
$$

Without loss of generality, we may assume that $\Delta_{0}$ is real. Note that the additive constant $D$ (which is equal to $\ell^{-1}(\infty)=\lim _{\omega \rightarrow \infty} \operatorname{Re} \Gamma(\omega)$ ) does not include $D_{V}$ : The forward scattering disorder does not affect the inverse localization length at large frequencies. If we first define $D_{R} \equiv D / 2$ and $D_{I} \equiv\left(1+\mu^{-2}\right) D / 2$, the confluent limit of the hypergeometric function $F$ may be taken by letting $\mu \rightarrow \infty$. Then, the hypergeometric function reduces to the generalized hypergeometric function ${ }_{0} F_{1}\left(1-i \omega / \tilde{D} ; \Delta_{0}^{2} / 4 \tilde{D}^{2}\right)$, which can be expressed in terms of the modified (hyperbolic) Bessel function $I_{p}(x)$. One finds (A\&S, Eq. (9.6.47))

$$
\lim _{\mu \rightarrow \infty} F=\left(\frac{\Delta_{0}}{2 \tilde{D}}\right)^{i \omega / D} \Gamma(1-i \omega / \tilde{D}) I_{-i \omega / \tilde{D}}\left(\frac{\Delta_{0}}{\tilde{D}}\right)
$$

Similarly, one can show that

$$
\lim _{\mu \rightarrow \infty} z F^{\prime}=\left(\frac{\Delta_{0}}{2 \tilde{D}}\right)^{1+i \omega / \tilde{D}} \Gamma(1-i \omega / \tilde{D}) I_{1-i \omega / \tilde{D}}\left(\frac{\Delta_{0}}{\tilde{D}}\right)
$$

In the incommensurate case, $\Gamma(\omega)$ therefore simplifies to

$$
\Gamma(\omega)=D-i \omega+\Delta_{0} \frac{I_{1-i \omega / \tilde{D}}\left(\frac{\Delta_{0}}{\tilde{D}}\right)}{I_{-i \omega / \tilde{D}}\left(\frac{\Delta_{0}}{\tilde{D}}\right)} .
$$

Using the formula (A\&S, Eq. (9.6.28))

$$
I_{1+v}(x)=I_{v}^{\prime}(x)-\frac{v}{x} I_{v}(x),
$$


Eq. (3.71) can also be written as

$$
\Gamma(\omega)=D+\Delta_{0} \frac{I_{-i \omega / \tilde{D}}^{\prime}\left(\frac{\Delta_{0}}{\tilde{D}}\right)}{I_{-i \omega / \tilde{D}}\left(\frac{\Delta_{0}}{\tilde{D}}\right)}
$$

Taking the imaginary part of Eq. (3.71) and making use of the Wronski relation (A\&S, Eq. (9.6.14))

$$
I_{v}(x) I_{1-v}(x)-I_{1+v}(x) I_{v}(x)=-2 \sin (\pi v) / \pi x,
$$

we find for the integrated DOS

$$
\mathcal{N}(\omega)=\frac{\tilde{D}}{\pi} \sinh \left(\frac{\pi \omega}{\tilde{D}}\right) \frac{\rho_{0}}{\left|I_{i \omega / \tilde{D}}\left(\frac{\Delta_{0}}{\tilde{D}}\right)\right|^{2}}
$$

This expression agrees with Ref. [32]. A plot of the DOS for different values of the parameter $v=\Delta_{0} / 2 D$ is shown at the top of Fig. 3.3 There is no Dyson singularity, and in the absence of a static gap $\Delta_{0}$, the disorder has no effect on the DOS so that $\rho(\omega)=\rho_{0}$ for $\nu=0$. At zero frequency, $\rho(0)$ is always finite and the DOS vanishes with increasing $v$, i.e. decreasing disorder, as $\rho(0)=\rho_{0} /\left[I_{0}(2 v)\right]^{2}$. Putting it the other way round, the disorder leads to a filling of the gap. As in the commensurate case, in the limit $D \rightarrow 0$, i.e. $v \rightarrow \infty$, the DOS reduces to the mean-field result $\rho(\omega)=$ $\rho_{0} \theta\left(\omega^{2}-\Delta_{0}^{2}\right)|\omega| /\left(\omega^{2}-\Delta_{0}^{2}\right)^{1 / 2}$.

Taking the real part of Eq. (3.71), we get for the inverse localization length

$$
\ell^{-1}(\omega)=D+\Delta_{0} \frac{I_{i \omega / \tilde{D}}\left(\frac{\Delta_{0}}{\tilde{D}}\right) I_{1-i \omega / \tilde{D}}\left(\frac{\Delta_{0}}{\tilde{D}}\right)+I_{-i \omega / \tilde{D}}\left(\frac{\Delta_{0}}{\tilde{D}}\right) I_{1+i \omega / \tilde{D}}\left(\frac{\Delta_{0}}{\tilde{D}}\right)}{2\left|I_{i \omega / \tilde{D}}\left(\frac{\Delta_{0}}{\tilde{D}}\right)\right|^{2}} .
$$

In contrast to the commensurate case, the localization length at $\omega=0$ is finite for any $\Delta_{0}$ and given by

$$
\ell^{-1}(0)=D+\Delta_{0} \frac{I_{1}\left(\frac{\Delta_{0}}{\tilde{D}}\right)}{I_{0}\left(\frac{\Delta_{0}}{\tilde{D}}\right)}
$$

While for $\Delta_{0} / \tilde{D} \rightarrow 0$ one has $\ell^{-1}(0) \rightarrow D$ (and also $\ell^{-1}(\omega) \rightarrow D$ for every $\omega$ ), for $\Delta_{0} / \tilde{D} \gg 1$ one finds $\ell^{-1}(\omega) \sim \Delta_{0}+D / 2-D_{V} / 2$. Finally, for small $D$ and $D_{V}$, the inverse localization length almost vanishes for $|\omega|>\Delta_{0}$. The weak disorder also creates a few localized states with energies $|\omega|<\Delta_{0}$ whose inverse localization length is given by $\ell^{-1}(\omega)=\sqrt{\Delta_{0}^{2}-\omega^{2}}$. A plot of the inverse localization length for different values of the parameter $v$ and $D_{V}=0$ is given at the bottom of Fig. 3.3. 

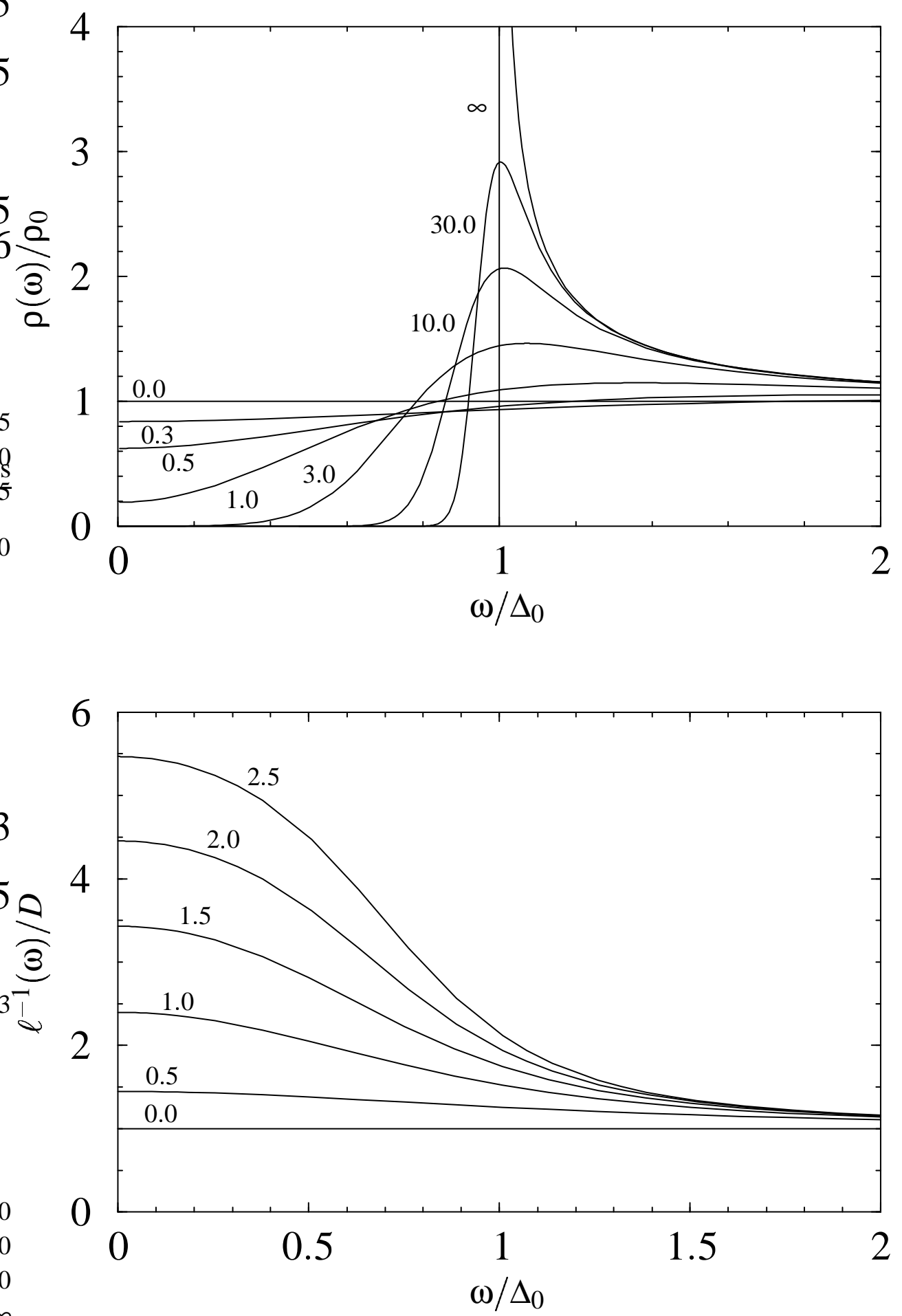

Figure 3.3: The DOS $\rho(\omega)$ and the inverse localization length $\ell^{-1}(\omega)$ in the white noise limit for the incommensurate case plotted versus $\omega / \Delta_{0}$ for $v=\Delta_{0} / 2 D=$ $0.0,0.3,0.5,1.0,3.0,10.0,30.0, \infty$ or $v=\Delta_{0} / 2 D=0.0,0.5,1.0,1.5,2.0,2.5$, respectively. 


\subsection{Infinite correlation lengths}

In the limit of infinite correlation lengths, $\Delta(x)$ becomes independent of $x$ and exact results for the averaged DOS of the FGM may be obtained. The limit of large correlation lengths $\xi$ is of special importance for Peierls systems because the correlation length of the order parameter diverges at the Peierls transition. Sadovskii was the first to consider the fluctuating gap model (FGM) with infinite correlation lengths [77] and calculated the one-electron Green function for the incommensurate case by summing up all diagrams in the perturbation expansion. This Green function leads to a DOS which exhibits a pseudogap at the Fermi energy. The commensurate case was later solved by Wonneberger and Lautenschlager [96].

\section{Taking the ensemble average}

The limit of infinite correlation lengths can be solved by averaging the desired quantity calculated with a static gap $\Delta$ over an appropriate probability distribution of $\Delta$. This amounts to taking an ensemble average.

\subsubsection{The commensurate case}

For real $\Delta$ and Gaussian statistics we have

$$
\langle\ldots\rangle=\int_{-\infty}^{\infty} \frac{d \Delta}{\sqrt{2 \pi \Delta_{s}^{2}}} e^{-\Delta^{2} / 2 \Delta_{s}^{2}} \ldots .
$$

The DOS can now be calculated as follows:

$$
\begin{aligned}
\rho_{\infty}(\omega) & =\rho_{0} \int_{-\omega}^{\omega} \frac{d \Delta}{\sqrt{2 \pi \Delta_{s}^{2}}} e^{-\Delta^{2} / 2 \Delta_{s}^{2}} \frac{\omega}{\sqrt{\omega^{2}-\Delta^{2}}} \\
& =2 \rho_{0} \frac{\omega}{\Delta_{s}} \frac{1}{\sqrt{2 \pi}} \int_{0}^{1} d u e^{-\left(\omega^{2} / 2 \Delta_{s}^{2}\right) u^{2}} \frac{1}{\sqrt{1-u^{2}}} \\
& =\rho_{0} \sqrt{\frac{\pi}{2}} \frac{\omega}{\Delta_{s}} e^{-\omega^{2} / 4 \Delta_{s}^{2}} I_{0}\left(\frac{\omega^{2}}{4 \Delta_{s}^{2}}\right) .
\end{aligned}
$$

Here, $I_{0}(u)$ is the modified Bessel function with index 0 already encountered in the section on the white noise limit.

If we define the inverse localization length $\ell_{\infty}^{-1}(\omega)$ for $\xi=\infty$ by the Thouless formula, we have

$$
\partial_{\omega} \ell_{\infty}^{-1}(\omega)=-\left\langle\frac{\omega}{\sqrt{\Delta^{2}-\omega^{2}}} \theta\left(\Delta^{2}-\omega^{2}\right)\right\rangle
$$

such that

$$
\begin{aligned}
\ell_{\infty}^{-1}(\omega) & =\left\langle\sqrt{\Delta^{2}-\omega^{2}} \theta\left(\Delta^{2}-\omega^{2}\right)\right\rangle \\
& =\sqrt{\frac{2}{\pi}} \frac{\omega^{2}}{\Delta_{s}} \int_{1}^{\infty} d u e^{-\left(\omega^{2} / 2 \Delta_{s}^{2}\right) u^{2}} \sqrt{u^{2}-1}
\end{aligned}
$$




\subsubsection{Incommensurate case}

For complex $\Delta(x)$ and Gaussian statistics, the process of averaging can be written as

$$
\langle\ldots\rangle=\int \frac{d \Delta}{\sqrt{2 \pi \Delta_{s}^{2}}} e^{-\Delta^{2} / 2 \Delta_{s}^{2}} \ldots
$$

We therefore obtain for the DOS

$$
\begin{aligned}
\rho_{\infty}(\omega) & =\rho_{0} \int_{|\Delta|<\omega} \frac{d \operatorname{Re} \Delta d \operatorname{Im} \Delta}{\pi \Delta_{s}^{2}} e^{-|\Delta|^{2} / \Delta_{s}^{2}} \frac{\omega}{\sqrt{\omega^{2}-|\Delta|^{2}}} \\
& =\rho_{0} \frac{\omega^{2}}{\Delta_{s}^{2}} \int_{0}^{1} d u e^{-\left(\omega^{2} / \Delta_{s}^{2}\right) u^{2}} \frac{1}{\sqrt{1-u^{2}}} \\
& =2 \rho_{0} \frac{\omega}{\Delta_{s}} e^{-\left(\omega^{2} / \Delta_{s}^{2}\right)} \operatorname{Erfi}\left(\frac{\omega}{\Delta_{s}}\right) .
\end{aligned}
$$

Here,

$$
\operatorname{Erfi}(u) \equiv \int_{0}^{u} e^{x^{2}} d x
$$

is the error function with an imaginary argument.

Similarly to the above calculations one can show that the inverse localization length for complex $\Delta(x)$ and Gaussian statistics is given by

$$
\ell_{\infty}^{-1}(\omega)=\Delta_{s} \frac{\sqrt{\pi}}{2} e^{-\omega^{2} / \Delta_{s}^{2}}
$$

Plots of $\rho_{\infty}(\omega)$ and $\ell_{\infty}^{-1}(\omega)$ are given in Fig. 3.4. While in the commensurate case the DOS vanishes linearly in $\omega$, it only vanishes quadratically in the incommensurate case. This is due to the fact that the probability distribution for complex $\Delta$ has less weight for small $|\Delta|$ than the one for real $\Delta$. The inverse localization length assumes for both the commensurate and the incommensurate case a finite value at $\omega=0$ and drops to zero as $\omega$ increases. That $\ell_{\infty}^{-1}(0)$ is finite in the commensurate case seems to contradict the general result $\ell_{\infty}^{-1}(0)=\Delta_{\mathrm{av}}=0$ derived in Chapter 2. One should keep in mind, however, that for $\xi=\infty$ we have only defined $\ell_{\infty}^{-1}(\omega)$ by $\operatorname{Re}\langle\mathcal{G}(x, x ; \omega)\rangle$. While for finite $\xi$ a single chain is representative for an ensemble of chains, for $\xi=\infty$, there is no self-averaging effect. On the other hand, it seems plausible to assume that the above results for $\xi=\infty$ give a good approximation to the case of finite $\xi$ if $\xi$ is much larger that any microscopic length scale involved. In particular, we have to demand $\Delta_{s} \xi \gg 1$ and $\omega \xi \gg 1$. The above results for the DOS and the inverse localization length at $\omega=0$ can therefore not be expected to hold for finite correlation lengths. In fact, we will see in the next chapter that for any finite $\xi$ we find in the commensurate case $\rho(0)=\infty$ and $\ell^{-1}(0)=0$. For $\Delta_{s} \xi \gg 1$ and $\omega \xi \gg 1$, however, we will find a remarkable agreement between the two solutions as predicted above. 

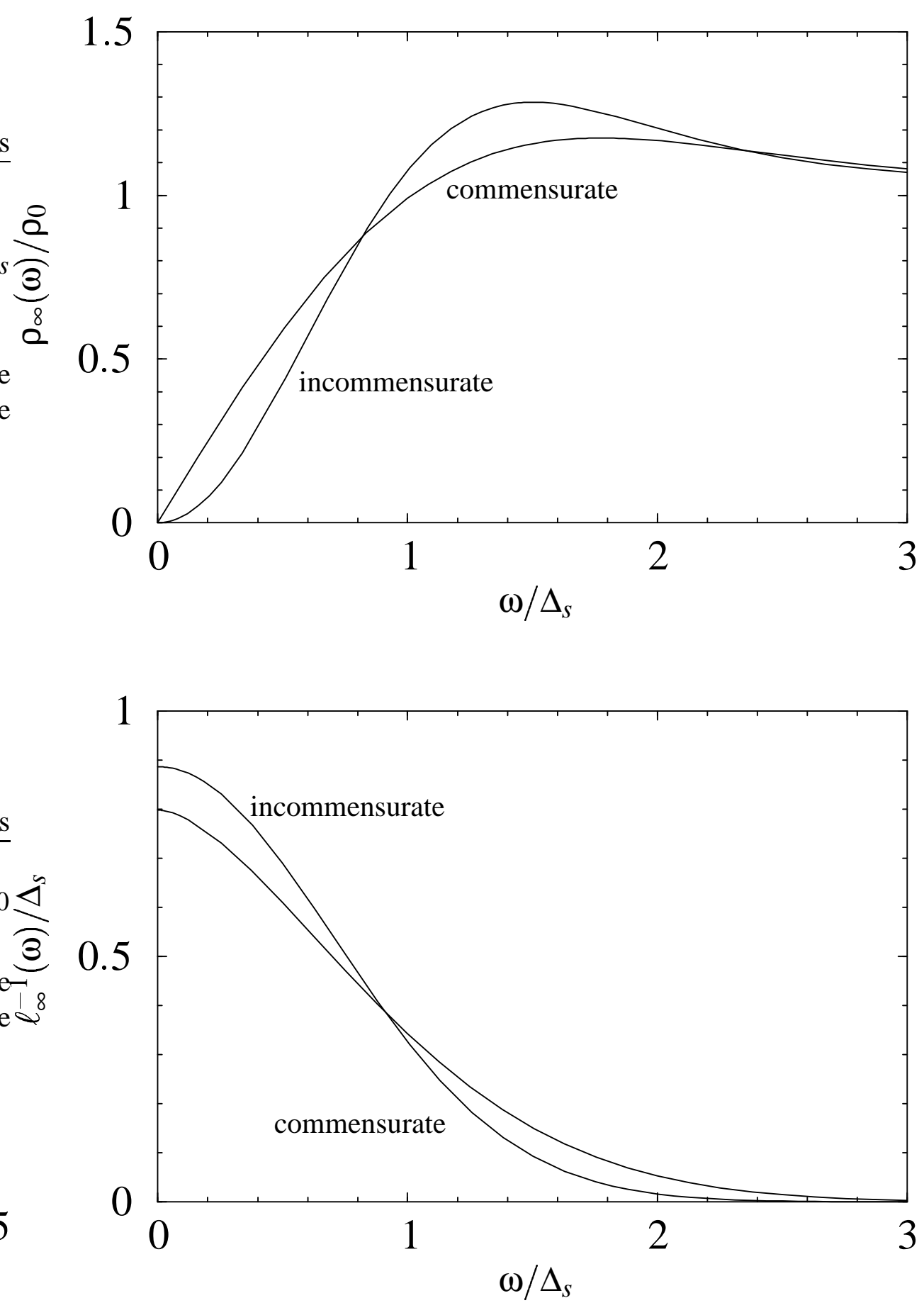

Figure 3.4: The DOS $\rho_{\infty}(\omega)$ and the inverse localization length $\ell_{\infty}^{-1}(\omega)$ for the commensurate and incommensurate case with $\xi=\infty$ and $\Delta_{\mathrm{av}}=0$. 



\section{Chapter 4}

\section{Finite correlation lengths}

While in the limit of very small and infinite correlation lengths $\xi$ of the random disorder, the fluctuating gap model (FGM) admits for an exact analytic calculation of the density of states (DOS) and the inverse localization length, in the intermediate regime of finite $\xi$ there are only approximate solutions available. It especially turns up the question: "How accurate are Sadovskii's solutions [78], which for a long time were thought to be exact?" An answer to this question is of particular interest because Sadovskii's solutions have become quite popular since the experimental discovery of a pseudogap in the underdoped cuprates above the critical temperature $T_{c}$ [80. 81]. In this chapter, we will calculate the DOS and the inverse localization length for Gaussian statistics, as approximately done by Sadovskii with very high accuracy numerically. We will also consider the case of only phase fluctuations for which we will even find an exact solution by applying a gauge transformation to the Green function and mapping the original problem onto a problem involving only white noise.

\subsection{Singularities in the density of states}

The exact results of the FGM derived in the white noise limit in the previous chapter imply under certain circumstances a Dyson singularity in the DOS. This singularity arises only in the commensurate case [i.e. for real $\Delta(x)$ ] and only if the forward scattering potential and $\Delta_{\mathrm{av}}=\langle\Delta(x)\rangle$ are sufficiently small [see Eqs. (3.44) and (3.46)]. Since the white noise limit describes the low-energy physics of physical systems characterized by small correlation lengths $\xi$, this statement should also be true for small but finite $\xi$. As far as I know, it was first shown by myself in collaboration with Peter Kopietz that the DOS $\rho(\omega)$ of the FGM exhibits a singularity at the Fermi energy for any finite value of the correlation length $\xi$ if the fluctuating order parameter field $\Delta(x)$ is real and its average $\langle\Delta(x)\rangle$ is sufficiently small [8]. To detect the singularity, we applied the boundary condition $\Delta_{\mathrm{BC}}=V_{\mathrm{BC}}=0$, such that the complete spectrum turned 
out to be continuous [see Eq. (2.58) and its following remark] 1] The existence of the Dyson singularity can also be seen in the discrete case by considering the equation of motion (2.89) for $V(x)=0$ and real $\Delta(x)$ which after the shift $\varphi \rightarrow \varphi-\pi / 2$ reads

$$
\partial_{x} \varphi(x)=2 \omega+2 \Delta(x) \sin \varphi(x) .
$$

The Dyson singularity in the DOS is due to phase resonance: If $\omega$ is small (compared to $\Delta_{s}, \Delta_{s}^{2} \xi$ and $\xi^{-1}$ ) but positive, the change of $\varphi(x)$ is dominated by the fluctuating term $2 \Delta(x) \sin \varphi(x)$. Only near $\varphi(x)=n \pi$ (with $n$ an integer) we have $\partial_{x} \varphi(x)=2 \omega>0$, such that $\varphi(x)$ can only grow on average. As $(\varphi(x)-n \pi) \approx \omega / \Delta_{s}$, fluctuation effects of $\Delta(x)$ become important, driving $\varphi(x)$ from $n \pi+\omega / \Delta_{s}$ to $(n+1) \pi-\omega / \Delta_{s}$. Near $\varphi(x)=(n+1) \pi$, the constant force $2 \omega$ dominates again and the above picture repeats itself.

As we decrease $\omega$, the "time" (which corresponds to the space coordinate $x$ ) to move $\varphi(x)$ from $n \pi-\omega / \Delta_{s}$ to $n \pi+\omega / \Delta_{s}$ will not change, but fluctuations of $\Delta(x)$ will need slightly longer to drive $\varphi(x)$ from $n \pi+\omega / \Delta_{s}$ to $(n+1) \pi-\omega / \Delta_{s}$, implying that $\varphi(x)$ decreases more slowly than $\omega$ as $\omega$ decreases. Now, the average DOS for frequencies between 0 and $\omega$ is given by

$$
\rho(\zeta \omega)=\frac{\mathcal{N}(\omega)}{\omega}=\lim _{x \rightarrow \infty} \frac{\varphi_{\omega}(x)}{2 \pi \omega x}
$$

where $\zeta$ is a number between 0 and 1 . Letting $\omega$ approach zero, it follows $\rho(0)=\infty$. This divergence describes the Dyson singularity in the DOS. The above reasoning is independent of the probability distribution of $\Delta(x)$. However, it should be noted that $\Delta(x)$ must not be dominated by one sign. If $\varphi(x) \approx n \pi$ and $(-1)^{n} \Delta(x)$ is negative, $\varphi(x)$ will fluctuate around the stable position near $n \pi+\omega / \Delta_{s} . \varphi(x)=(n+1) \pi$ can only be reached if $(-1)^{n} \Delta(x)$ is positive on average over a finite interval. We therefore conclude that we expect a Dyson singularity if $\Delta(x)$ is real and fluctuates around $\Delta_{\mathrm{av}} \equiv$ $\langle\Delta(x)\rangle$ with $\Delta_{\text {av }}$ sufficiently small.

For complex $\Delta(x)$, fluctuations of the phase of $\Delta(x)$ can be mapped via the gauge transformation (2.98) onto a forward scattering potential. Since the amplitude $|\Delta(x)|$ is always positive and the phase fluctuations lead to an effective local shift of the frequency $\omega$, there should be no Dyson singularity. Instead, we expect a suppression of the DOS, i.e. a pseudogap.

\subsection{Numerical algorithm}

In the following, we present an exact algorithm which for stepwise constant potentials allows for simultaneous numerical calculations of the integrated DOS and the inverse

\footnotetext{
${ }^{1}$ Our results have been turned into question in Ref. [67], but as pointed out in Ref. [12] and as will be shown below, the DOS diverges in the commensurate case for $\langle\Delta(x)\rangle=0$ and $V(x)=0$ for any finite $\xi$ at the Fermi energy.
} 
localization length. By choosing the step size sufficiently small, the integrated DOS and the inverse localization length may be calculated for arbitrary given potentials. Let us partition the interval $(0, L)$ into $N$ intervals $\left(x_{n}, x_{n+1}\right)$ of length $\delta_{n}=x_{n+1}-x_{n}$ with $x_{0}=0<x_{1}<\ldots<x_{N}=L$, such that $\Delta(x) \equiv \Delta_{n}$ and $V(x) \equiv V_{n}$ for $x_{n}<x<x_{n+1}$. Let us also define $\tilde{\omega}_{n}$ as $\tilde{\omega}_{n} \equiv \omega-V_{n}$.

To find an exact analytic solution for the given stepwise constant potentials of the equations of motion (2.89) and (2.90), let us consider again the related $S$-matrix $S(L, 0 ; \omega)$, which can be written as the finite product

$$
S(L, 0 ; \omega)=\prod_{n=0}^{N-1} S_{n} \equiv \prod_{n=0}^{N-1} S\left(x_{n+1}, x_{n} ; \omega\right),
$$

where the $S_{n}$ are given by [see Eqs. (2.54) and (2.55)]

$$
\begin{aligned}
S_{n}= & \cosh \left[\sqrt{\left|\Delta_{n}\right|^{2}-\tilde{\omega}_{n}^{2}} \delta_{n}\right] \sigma_{0} \\
& +i \sinh \left[\sqrt{\left|\Delta_{n}\right|^{2}-\tilde{\omega}_{n}^{2}} \delta_{n}\right] \frac{\tilde{\omega}_{n} \sigma_{3}+\Delta_{n} \sigma_{+}-\Delta_{n}^{*} \sigma_{-}}{\sqrt{\left|\Delta_{n}\right|^{2}-\tilde{\omega}_{n}^{2}}}, \quad \tilde{\omega}_{n}^{2}<\left|\Delta_{n}\right|^{2}, \\
S_{n}= & \cos \left[\sqrt{\tilde{\omega}_{n}^{2}-\left|\Delta_{n}\right|^{2}} \delta_{n}\right] \sigma_{0} \\
& +i \sin \left[\sqrt{\tilde{\omega}_{n}^{2}-\left|\Delta_{n}\right|^{2}} \delta_{n}\right] \frac{\tilde{\omega}_{n} \sigma_{3}+\Delta_{n} \sigma_{+}-\Delta_{n}^{*} \sigma_{-}}{\sqrt{\tilde{\omega}_{n}^{2}-\left|\Delta_{n}\right|^{2}}}, \quad \tilde{\omega}_{n}^{2}>\left|\Delta_{n}\right|^{2} .
\end{aligned}
$$

Eq. (4.3) implies the recurrence relation

$$
S\left(x_{n+1}, 0 ; \omega\right)=S_{n} S\left(x_{n}, 0 ; \omega\right),
$$

which we will now cast into recurrence relations for $\varphi(x)$ and $\zeta(x)$ : Taking the logarithm of

$$
S_{22}\left(x_{n+1}, 0\right)=\left(S_{n}\right)_{22} S_{22}\left(x_{n}, 0\right)+\left(S_{n}\right)_{21} S_{12}\left(x_{n}, 0\right),
$$

we get

$$
\ln S_{22}\left(x_{n+1}, 0\right)=\ln S_{22}\left(x_{n}, 0\right)+\ln \left[\left(S_{n}\right)_{22}+\left(S_{n}\right)_{21} S_{12}\left(x_{n}, 0\right) / S_{22}\left(x_{n}, 0\right)\right] .
$$

Recalling

$$
\begin{aligned}
& S_{12}(x, 0)=\exp [i \varphi(x) / 2+\zeta(x) / 2], \\
& S_{22}(x, 0)=\exp [-i \varphi(x) / 2+\zeta(x) / 2],
\end{aligned}
$$

the recurrence relation for the $S$-matrix is transformed into the recurrence relations for $\varphi_{n} \equiv \varphi\left(x_{n}\right)$ and $\zeta_{n} \equiv \zeta\left(x_{n}\right)$,

$$
\begin{aligned}
& \varphi_{n+1}=\varphi_{n}-2 \operatorname{Im}\left[\ln z_{n}\right], \\
& \zeta_{n+1}=\zeta_{n}+2 \operatorname{Re}\left[\ln z_{n}\right],
\end{aligned}
$$

\footnotetext{
${ }^{2}$ It should be obvious that $\widetilde{\omega}_{n} \equiv \omega-V_{n}$ is not a Matsubara frequency.
} 
where we have introduced

$$
z_{n}=\left(S_{n}\right)_{22}+\left(S_{n}\right)_{21} \exp \left(i \varphi_{n}\right)
$$

Note that Eqs. (4.11) and (4.12) are integrated forms of the equations of motion (2.89) and (2.90). The real and imaginary part of $\ln z_{n}$ can be determined by using the formula

$$
\ln z_{n} \equiv \ln \left(\left|z_{n}\right| e^{i \alpha_{n}}\right)=\ln \left|z_{n}\right|+i \alpha_{n} \equiv \ln \left|z_{n}\right|+i \arg \left(z_{n}\right)
$$

The argument of $z_{n}, \arg \left(z_{n}\right)$ can be obtained up to a multiple of $2 \pi$ from 3

$$
\arg \left(z_{n}\right)=2 \pi m_{n}+\operatorname{sgn}\left[\operatorname{Im} z_{n}\right] \arccos \left(\frac{\operatorname{Re} z_{n}}{\left|z_{n}\right|}\right)
$$

To find the integer

$$
m_{n}=\left[\frac{\arg \left(z_{n}\right)}{2 \pi}+\frac{1}{2}\right]_{\mathrm{int}},
$$

we define $z_{n}(x)$ by $z_{n}$ with $\delta_{n}$ replaced by $x-x_{n} . \quad z_{n}(x)$ is an analytic function of $x$ and at $x=x_{n+1}$ agrees with $z_{n}$. For $\tilde{\omega}_{n}^{2}<\left|\Delta_{n}\right|^{2}$, it follows from Eq. (4.4) that $\operatorname{Im}\left[z_{n}(x)\right] \propto \sinh \left[\sqrt{\left|\Delta_{n}\right|^{2}-\tilde{\omega}_{n}^{2}}\left(x-x_{n}\right)\right]$ does not change its sign for any $x>x_{n}$, such that $\left|\arg \left[z_{n}(x)\right]\right|<\pi$ and $m_{n}$ has to be zero. For $\tilde{\omega}_{n}^{2}>$ $\left|\Delta_{n}\right|^{2}$, however, $\operatorname{Im}\left[z_{n}(x)\right] \propto \sin \left[\sqrt{\tilde{\omega}_{n}^{2}-\left|\Delta_{n}\right|^{2}}\left(x-x_{n}\right)\right]$, such that $\left|\left[\arg \left(z_{n}(x)\right) / \pi\right]_{\text {int }}\right|=$ $\left[\sqrt{\tilde{\omega}_{n}^{2}-\left|\Delta_{n}\right|^{2}}\left(x-x_{n}\right) / \pi\right]_{\text {int }}$. Since the constant of proportionality is negative for $\tilde{\omega}_{n}>\left|\Delta_{n}\right|$ and positive for $\widetilde{\omega}_{n}<-\left|\Delta_{n}\right|$, it follows

$$
m_{n}=\left[\frac{1}{2}-\frac{\operatorname{sgn}\left(\tilde{\omega}_{n}\right) \sqrt{\tilde{\omega}_{n}^{2}-\left|\Delta_{n}\right|^{2}} \delta_{n}}{2 \pi}\right]_{\mathrm{int}} .
$$

To summarize, we can simultaneously calculate the integrated DOS and the inverse localization length for arbitrary stepwise constant potentials using the following iterative algorithm with the initial values $\varphi_{0}=\zeta_{0}=0$,

$$
\begin{aligned}
\varphi_{n+1} & =\varphi_{n}-2\left[2 \pi m_{n}+\operatorname{sgn}\left[\operatorname{Im} z_{n}\right] \arccos \left(\frac{\operatorname{Re} z_{n}}{\left|z_{n}\right|}\right)\right] \\
\zeta_{n+1} & =\zeta_{n}+2 \ln \left|z_{n}\right|
\end{aligned}
$$

where $z_{n}$ and $m_{n}$ are given by

\footnotetext{
${ }^{3}$ When implementing the algorithm on a computer and using the language $\mathrm{C}, \arg \left(z_{n}\right)$ is given up to a multiple of $2 \pi$ by the function atan $2\left(\operatorname{Im} z_{n}, \operatorname{Re} z_{n}\right)$, which returns a value between $-\pi$ and $\pi$.
} 


$$
\begin{aligned}
& z_{n}=\left(S_{n}\right)_{22}+\left(S_{n}\right)_{21} \exp \left(i \varphi_{n}\right), \\
& m_{n}= \begin{cases}{\left[\frac{1}{2}-\frac{\operatorname{sgn}\left(\tilde{\omega}_{n}\right) \sqrt{\tilde{\omega}_{n}^{2}-\left|\Delta_{n}\right|^{2}} \delta_{n}}{2 \pi}\right]_{\text {int }},} & \tilde{\omega}_{n}^{2}-\left|\Delta_{n}\right|^{2}>0 \\
0 & , \quad \tilde{\omega}_{n}^{2}-\left|\Delta_{n}\right|^{2} \leq 0\end{cases}
\end{aligned}
$$

and the matrix elements of $S_{n}$ are determined by Eqs. (4.4) and (4.5).

\subsubsection{Generation of disorder}

In case of finite correlation lengths, specific extensive physical quantities (which are obtained by relating extensive quantities to the length of the system) show a selfaveraging effect as the length of the chain increases [58], i.e. they become independent of the concrete realization of the disorder. This self-averaging effect can be understood by partitioning a very long macroscopic chain into a large number of chains, each one still being much longer than the correlation length and any other microscopic length scale. In this case, boundary effects between adjacent parts of the original chain may be neglected and we are practically left with an ensemble of a large number of independent chains. Physical quantities can now be calculated for each chain individually, assuming independently all possible values with their respective statistical weight. Specific extensive quantities are now given by the ensemble-average, giving a non-random value in the thermodynamic limit. The DOS and the inverse localization length can therefore be calculated by generating one typical very long chain.

\section{Gaussian disorder}

To generate Gaussian disorder at the sample points $x_{n}$ with the first two moments satisfying

$$
\langle\Delta(x)\rangle=\Delta_{\mathrm{av}} \quad, \quad\left\langle\tilde{\Delta}(x) \tilde{\Delta}\left(x^{\prime}\right)\right\rangle=\Delta_{s}^{2} e^{-\left|x-x^{\prime}\right| / \xi},
$$

where $\tilde{\Delta}(x) \equiv \Delta(x)-\Delta_{\text {av }}$, we use a realization of an Ornstein-Uhlenbeck process described in more general form in Appendix B] Using the Box-Muller algorithm [74], we generate independent Gaussian random numbers $g_{n}$ with $\left\langle g_{n}\right\rangle=0$ and $\left\langle g_{n}^{2}\right\rangle=1$. For real $\Delta(x)$, we generate $\tilde{\Delta}_{n}=\Delta_{n}-\Delta_{\text {av }}$ recursively by

$$
\tilde{\Delta}_{0}=\Delta_{s} g_{0}, \tilde{\Delta}_{n+1}=a_{n} \tilde{\Delta}_{n}+\sqrt{1-a_{n}^{2}} \Delta_{s} g_{n+1},
$$

where $a_{n}=e^{-\left|\delta_{n}\right| / \xi}$. It is shown in Appendix B that this Markov process indeed leads to a Gaussian random process with the desired correlation functions. The Markov property of the algorithm allows us to generate the disorder simultaneously with the iteration of the recurrence relations (4.18) and (4.19), so that the algorithm presented above practically needs no memory space and, in principle, arbitrary long chains can 
be considered. If we choose $\left|\delta_{n}\right| / \xi \ll 1$ (in practical calculations we choose $\left|\delta_{n}\right| / \xi \approx$ 0.0001 to 0.05 depending on $\Delta_{s} \xi$ and make sure that lessening of $\left|\delta_{n}\right| / \xi$ does not change the results), the (integrated) DOS and the inverse localization length may be calculated with arbitrary accuracy numerically.

Of course, the above algorithm can also be used to generate $V_{n}$ and in the complex case, $\operatorname{Re} \Delta_{n}$ and $\operatorname{Im} \Delta_{n}$ can be generated by replacing $\Delta_{s}$ by $\Delta_{s} / \sqrt{2}$ (see Appendix B).

\subsubsection{Results}

First numerical calculations of the DOS of the FGM in the regime of finite correlation lengths were done by myself in collaboration with Peter Kopietz using an algorithm similar to the one presented here [11]. Simultaneously, Millis and Monien presented their data obtained by an exact diagonalization of a lattice regularization of the FGM [66]. However, these authors did not make any attempts to relate their results to the continuous FGM which would have allowed for a more direct comparison with the solutions given by Sadovskii [78].

In contrast to the algorithm described in Ref. [11], the algorithm presented here does not only allow for a numerical calculation of the (integrated) DOS, it is also capable of a simultaneous evaluation of the localization length which for finite $\xi$ has never been published before.

\section{Commensurate case}

In Fig. 4.1, we show our numerical results for the DOS $\rho(\omega)$ and inverse localization length $\ell^{-1}(\omega)$ for real $\Delta(x)$ (with $\Delta_{\mathrm{av}}=0$ and $V(x)=0$ ), which refers to the symmetric phase of a commensurate system with no forward scattering. Except for $\Delta_{s} \xi=1000,0.2$ we have chosen the same values of the dimensionless parameter $\Delta_{s} \xi$ as in Fig. 7 of Ref. [78] 4 One clearly sees the Dyson singularity in the DOS which exists for any finite value of $\xi$ and overshadows the pseudogap at sufficiently small energies. One can also see that this Dyson singularity is accompanied by a singularity in the inverse localization length. The inverse localization length drops to zero at $\omega=0$, in accordance with the exact result $\ell^{-1}(0)=\left\langle\Delta_{\text {av }}\right\rangle$ [see Eq. [2.121)].

The Dyson singularity in the DOS is missed by Sadovskii's algorithm [78]. For a more quantitative description of the Dyson singularity we have plotted the logarithm of the integrated DOS $\mathcal{N} / \Delta_{s}$ versus the logarithm of $-\ln \left(\omega / \Delta_{s}\right)$. For frequencies between $\omega=10^{-11} \Delta_{s}$ and $\omega=10^{-6}$ we find that the data can be very well fitted by a straight line, such that

$$
\mathcal{N}(\omega)=\rho_{0} \frac{\Delta_{s} B(\xi)}{\left|\ln \left(\omega / \Delta_{s}\right)\right|^{\alpha(\xi)}},
$$

\footnotetext{
${ }^{4}$ Note that by choosing the length of the system to be $\Delta_{s} \xi=10^{8}$ which is ten times as large as in Ref. [11] we have improved the accuracy of the data of the DOS.
} 

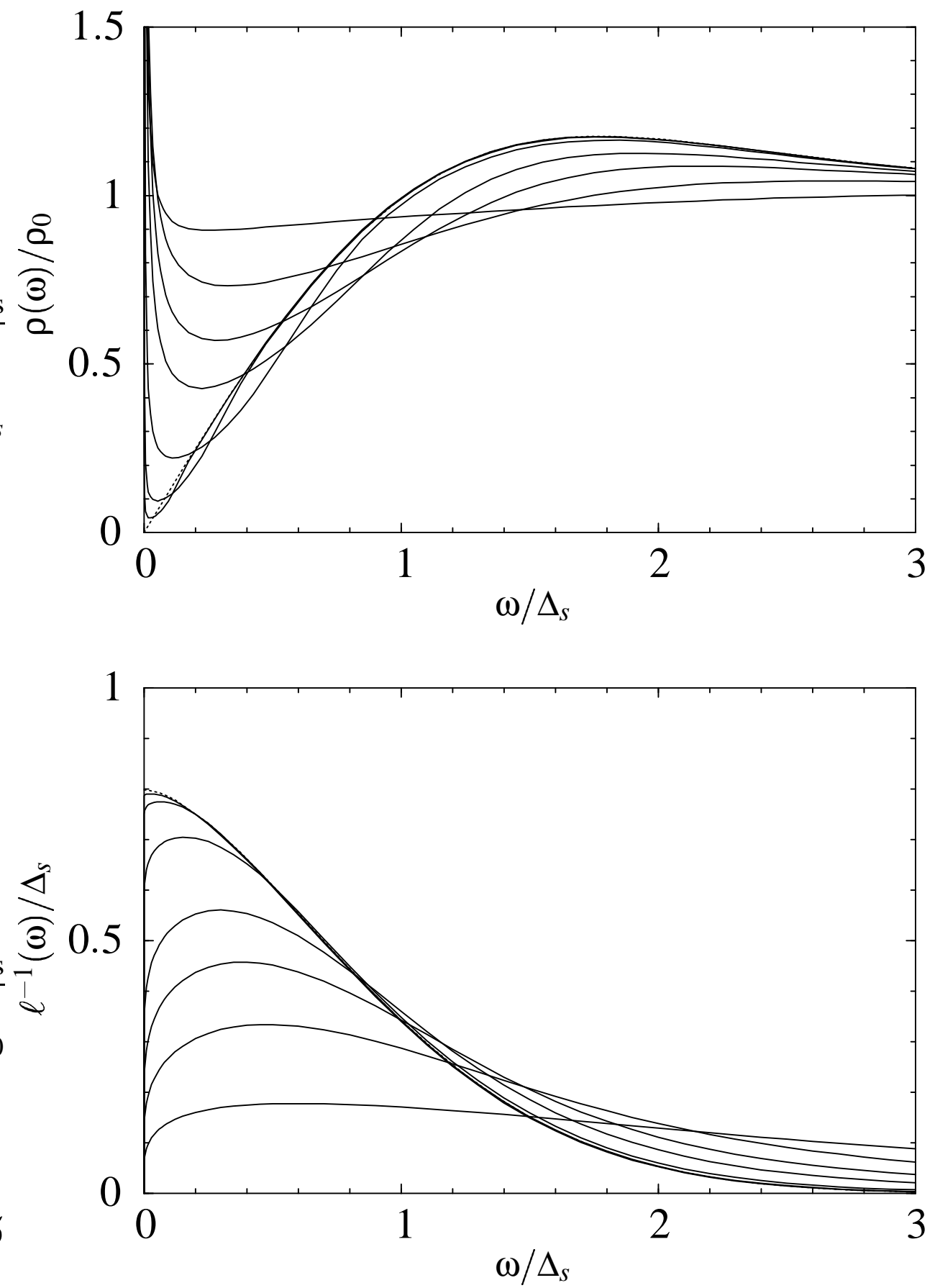

Figure 4.1: Plot of the DOS $\rho(\omega)$ and the inverse localization length $\ell^{-1}(\omega)$ for real $\Delta(x)$ with Gaussian statistics, $\Delta_{s} L=10^{8}$, and finite correlation lengths $\Delta_{s} \xi=$ $1000.0,100.0,10.0,2.0,1.0,0.5$, and 0.2 . As $\Delta_{s} \xi$ increases, the pseudogap in the DOS becomes deeper and the inverse localization increases for small frequencies. For any finite $\xi$, we find $\rho(0)=\infty$ and $\ell^{-1}(\omega)=0$. The dotted line represents the exact result derived in Chapter 3 and for $\omega \gtrsim 0.2 \Delta_{s}$ is almost indistinguishable from the result for $\Delta_{s} \xi=1000.0$. 


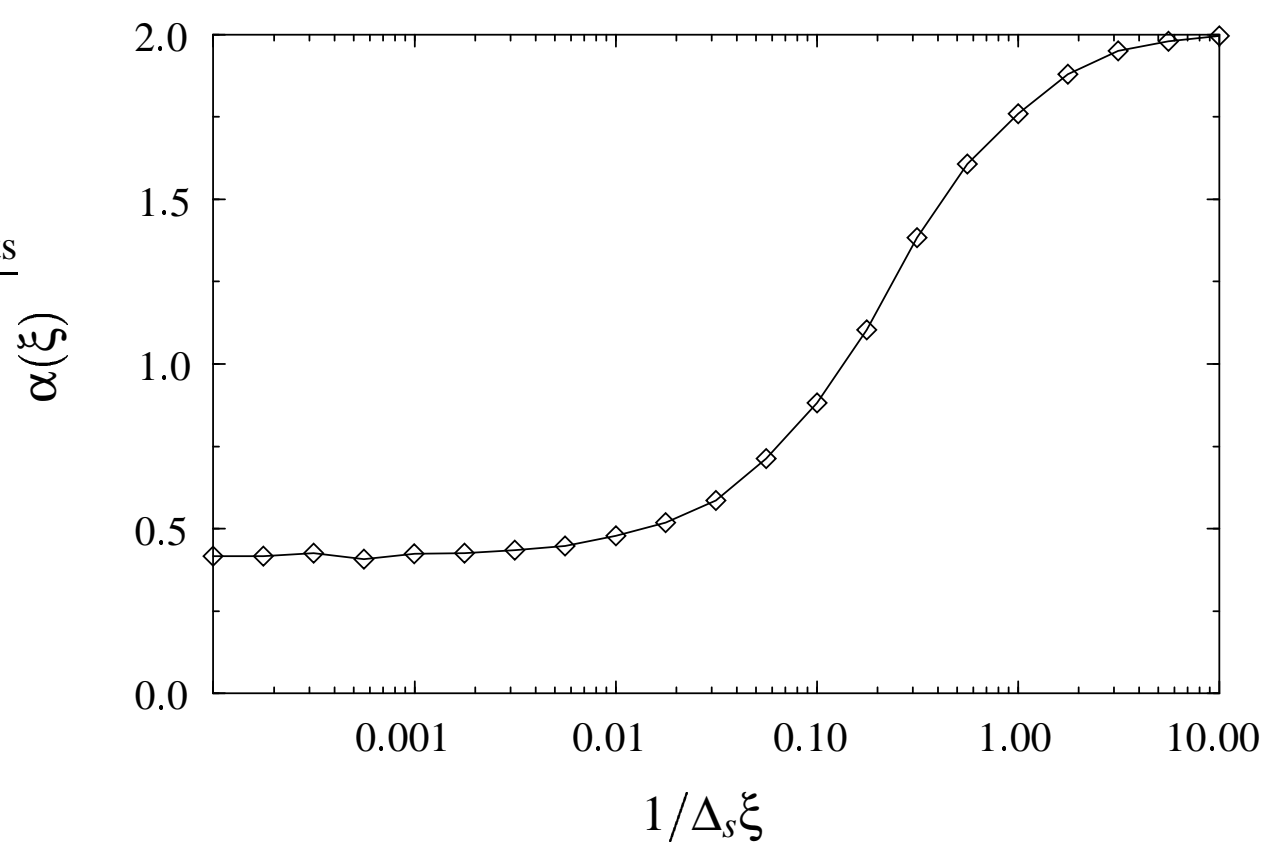

Figure 4.2: Plot of the exponent $\alpha(\xi)$ defined by Eq. (4.24) for frequencies between $\omega=10^{-6} \Delta_{s}$ and $\omega=10^{-11} \Delta_{s}$. While $\alpha(\xi)$ slightly overestimates the white noise result for small $\Delta_{s} \xi$, in the opposite limit $\Delta_{s} \xi \gg 1$ we find $\alpha \approx 0.41$.

which implies for the DOS

$$
\rho(\omega)=\rho_{0} \frac{A(\xi)}{\left(\omega / \Delta_{S}\right)\left|\ln \left(\omega / \Delta_{S}\right)\right|^{1+\alpha(\xi)}},
$$

with $A(\xi)=\alpha(\xi) B(\xi)$. Plots of the exponent $\alpha(\xi)$ and the weight factors of the Dyson singularity $A(\xi)$ and $B(\xi)$ are shown in Figs. 4.2 and 4.3 For $\Delta_{s} \xi \ll 1$ our data is consistent with the white noise result $\alpha=2$. As $\Delta_{s} \xi$ increases, the exponent $\alpha(\xi)$ decreases, assuming for large correlation lengths $\xi$ the finite value

$$
\alpha(\xi) \approx 0.41, \quad \Delta_{s} \xi \gtrsim 500 .
$$

Fitting the data for $A(\xi)$ in the regime between $\Delta_{s} \xi=500$ and $\Delta_{s} \xi=10000$ to a power law shows that the weight of the singularity of the DOS vanishes as

$$
A(\xi)=0.175\left(\Delta_{s} \xi\right)^{-0.65}
$$

The plot of the DOS given in Fig. 4.1 shows that for large correlation lengths $\xi$ the Dyson singularity only overshadows a pseudogap, such that $\rho(\omega)$ takes a minimal value at a certain frequency $\omega^{*}(\xi)$. A double-logarithmic plot of $\rho\left(\omega^{*}\right)$ versus $\left(\Delta_{s} \xi\right)^{-1}$ is given by the triangles in Fig. 4.4 The straight line gives a fit to a power-law: 


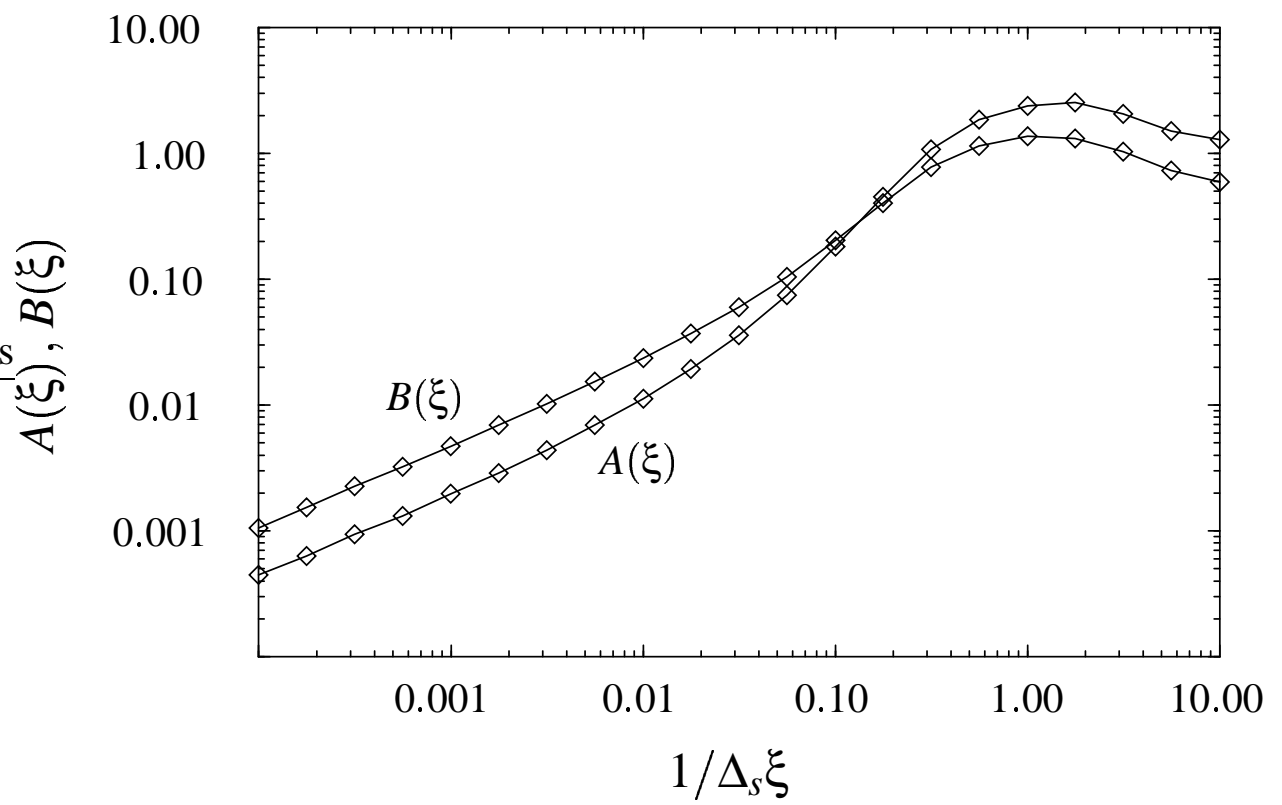

Figure 4.3: Plot of the weight factors $A(\xi)$ and $B(\xi)$ of the Dyson singularity defined by Eqs. (4.24) and 4.25) for frequencies between $\omega=10^{-6} \Delta_{s}$ and $\omega=10^{-11} \Delta_{s}$. As the correlation length increases both weight factors scale to zero.

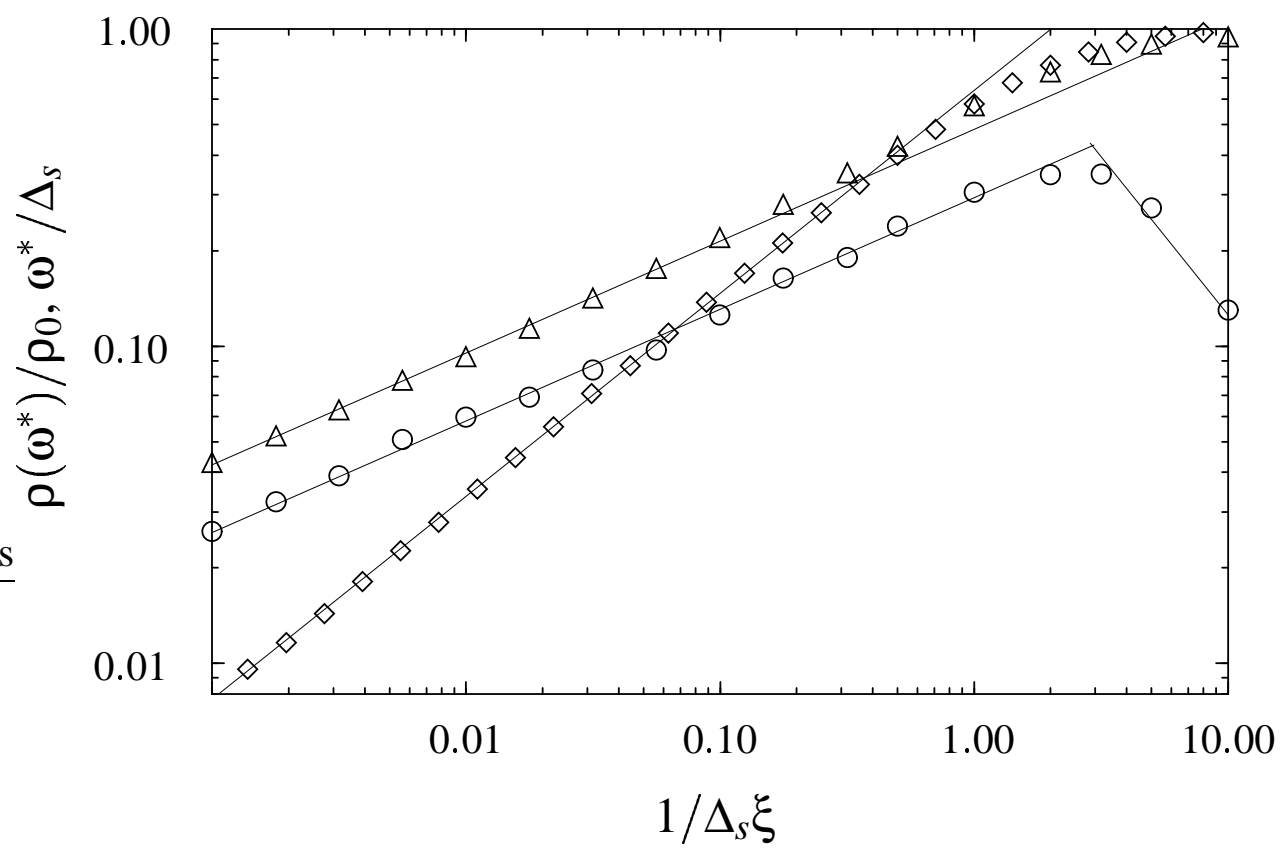

Figure 4.4: Double-logarithmic plot of $\rho\left(\omega^{*}\right) / \rho_{0}$ as a function of $1 / \Delta_{s} \xi$ for real $\Delta(x)$ (triangles) and complex $\Delta(x)$ (diamonds), where $\omega^{*}$ is the energy for which the DOS assumes its minimum. While $\omega^{*}=0$ for complex $\Delta(x)$, the circles give the doublelogarithmic plot of $\omega^{*} / \Delta_{s}$ for real $\Delta(x)$ as a function of $1 / \Delta_{s} \xi$. 


$$
\rho\left(\omega^{*}\right) / \rho_{0}=C\left(\Delta_{s} \xi\right)^{-\mu}
$$

We find

$$
C=0.482 \pm 0.010, \quad \mu=0.3526 \pm 0.0043
$$

The circles in Fig. 4.4 show $\omega^{*}$ where $\rho(\omega)$ is minimal. The long solid line is a fit to a power-law

$$
\omega^{*} / \Delta_{s}=D\left(\Delta_{s} \xi\right)^{-\gamma}
$$

Here, we find

$$
D=0.2931 \pm 0.0074, \quad \gamma=0.3513 \pm 0.0051 \text {. }
$$

such that within numerical accuracy $\mu=\gamma$. The proportionality of $\rho\left(\omega^{*}\right)$ to the energy scale $\omega^{*}$, which can be interpreted as the width of the Dyson singularity, can also directly be seen in Fig. 4.1, Finally we note that for $\Delta_{s} \xi \lesssim 0.2$ our algorithm produces results consistent with the white noise limit $\Delta_{s} \xi \ll 1$. From the exact solution of Ovchinnikov and Erikhman [71] we obtain $\rho\left(\omega^{*}\right) / \rho_{0} \rightarrow 0.9636$ and $\omega^{*} \rightarrow 1.2514 \Delta_{s}^{2 \xi}$ which determines the short solid line in Fig. 4.4, describing $\omega^{*}(\xi)$ in the white-noise limit.

\section{Incommensurate case}

The DOS $\rho(\omega)$ and inverse localization length $\ell^{-1}(\omega)$ for complex $\Delta(x)$ (and $\Delta_{\mathrm{av}}=$ $V(x)=0$ ), which refers to the symmetric phase of the commensurate case with no forward scattering are presented in Fig. 4.5. Neither the DOS nor the inverse localization length involve a singularity. In fact, a direct comparison of our results for the DOS with those obtained from Sadovskii's algorithm shows a good agreement.

For a more quantitative comparison, the diamonds in Fig. 4.4 show the DOS $\rho(0)$ at the Fermi energy. A fit to a power-law gives

$$
\rho(0) / \rho_{0}=C\left(\Delta_{s} \xi\right)^{-\mu}
$$

with

$$
C=0.6397 \pm 0.0066, \quad \mu=0.6397 \pm 0.0024 \text {. }
$$

Note that within numerical accuracy we find $C=\mu$. This result should be compared with Sadovskii's approximate result $C=0.541 \pm 0.013$ and $\mu=1 / 2$.

\subsection{Phase fluctuations only}

As pointed out by Grüner [37], far below the mean-field critical temperature $T_{c}^{\mathrm{MF}}$, amplitude fluctuations of the complex order parameter $\Delta(x) \equiv|\Delta(x)| e^{i \vartheta(x)}$ are gradually frozen out, and the amplitude $|\Delta(x)|$ is close to its $T=0$ value (with the Boltzmann constant $k_{B}$ set equal to one)

$$
\Delta_{s} \equiv \Delta_{0}(0)=1.764 T_{c}^{\mathrm{MF}} .
$$



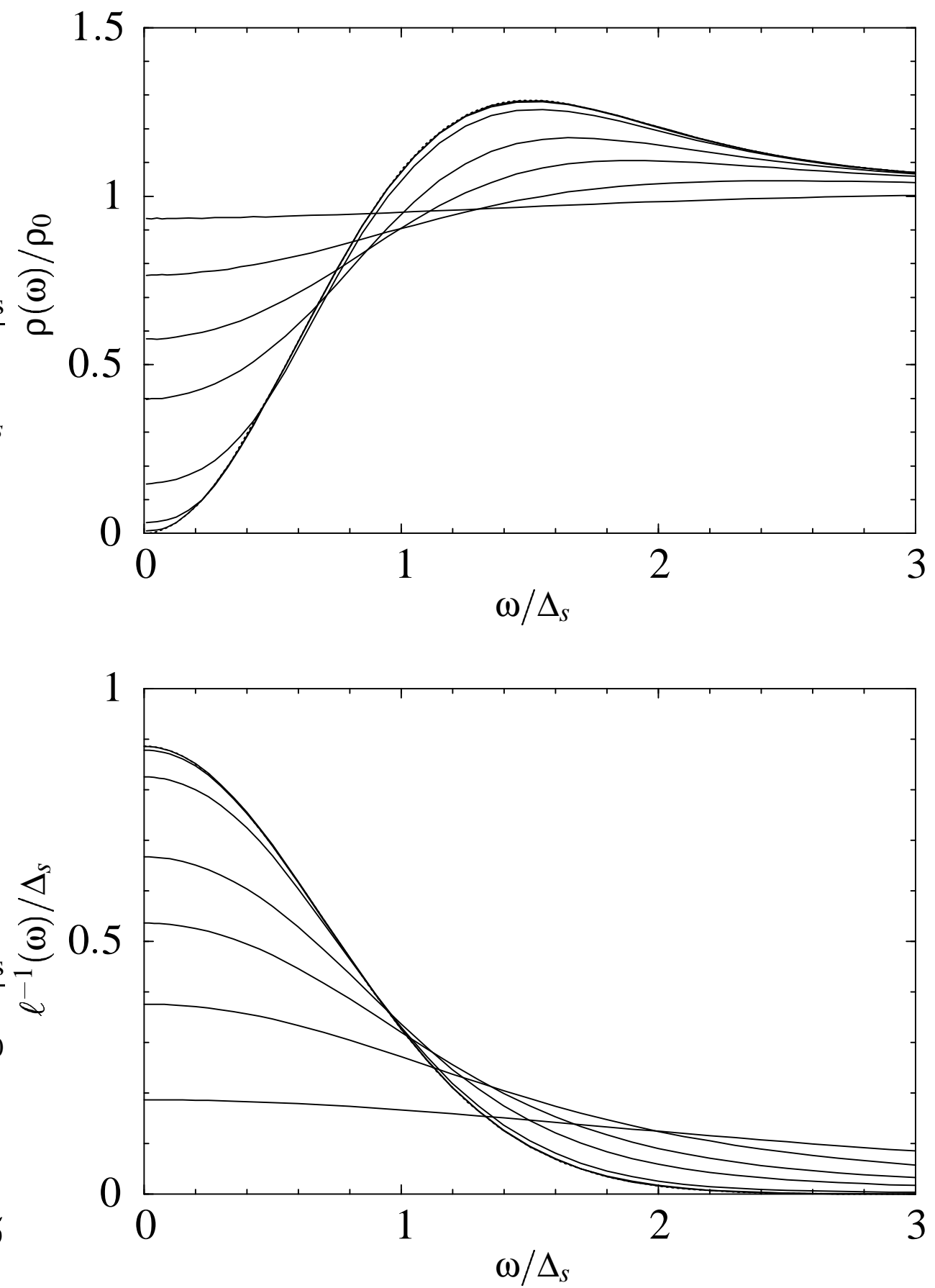

Figure 4.5: Plot of the DOS $\rho(\omega)$ and the inverse localization length $\ell^{-1}(\omega)$ for complex $\Delta(x)$ with Gaussian statistics, $\Delta_{s} L=10^{8}$, and finite correlation lengths $\Delta_{s} \xi=1000.0,100.0,10.0,2.0,1.0,0.5$, and 0.2 . As $\Delta_{s} \xi$ increases, the pseudogap in the DOS becomes deeper and the inverse localization increases for small frequencies. The dotted line represents the exact result derived in Chapter 3 This line is hardly recognizable because it is almost indistinguishable from the line for $\Delta_{s} \xi=1000.0$. 
Long-wave length fluctuations of the phase, however, cost only little energy, so that the order parameter fluctuates at the bottom of the two-dimensional free energy potential well, which has the shape of a "Mexican hat". Ignoring quartic terms in the gradient expansion of the free energy (which can be found in Appendix A), the free energy is given for a constant amplitude [see Eq. (1.120) and Ref. [14]] by

$$
F^{(\text {phase })}\{V\}=\frac{1}{2} s \rho_{s}(T) \int_{0}^{L} d x V^{2}(x)
$$

The superfluid density $\rho_{s}(T)$ was given in Eq. 11.122) and at low temperatures is approximately equal to $\rho_{0}$. In terms of the superfluid "velocity" $V(x)=\partial_{x} \vartheta(x) / 2$, the process of averaging can be written as

$$
\langle\ldots\rangle=\frac{\int \mathcal{D}\{V\} \ldots e^{-\beta F\{V\}}}{\int \mathcal{D}\{V\} e^{-\beta F\{V\}}}
$$

Since $F\{V\}$ is Gaussian and local, the process of averaging is described by Gaussian white noise. The first two moments of $V(x)$ are given by

$$
\begin{aligned}
\langle V(x)\rangle & =0, \\
\left\langle V(x) V\left(x^{\prime}\right)\right\rangle & =2 \frac{1}{4 \xi(T)} \delta\left(x-x^{\prime}\right),
\end{aligned}
$$

with [see Eq 1.128]

$$
\xi(T)=\frac{s \rho_{s}(T)}{2 T}
$$

Well below the mean-field temperature $T_{c}^{\mathrm{MF}}$, we can use the BCS gap equation $\Delta_{s}=$ $1.764 T_{c}^{\mathrm{MF}}$ and $\rho_{s}(T) \approx \rho_{0}=\pi^{-1}$ to get for the dimensionless parameter $5 \Delta_{s} \xi$

$$
\Delta_{s} \xi(T)=0.281 s T_{c}^{\mathrm{MF}} / T .
$$

As already shown in Chapter 1, Eqs. (4.37) and (4.38) imply the correlation functions

$$
\begin{aligned}
\langle\Delta(x)\rangle & =0, \quad\left\langle\Delta(x) \Delta\left(x^{\prime}\right)\right\rangle=0, \\
\left\langle\Delta(x) \Delta^{*}\left(x^{\prime}\right)\right\rangle & =\Delta_{s}^{2} \exp \left(-\left|x-x^{\prime}\right| / \xi(T)\right) .
\end{aligned}
$$

To calculate physical quantities like the DOS or the inverse localization length, we use the gauge invariance of these quantities under the gauge transformation (2.98) and map the phase fluctuations of the order parameter $\Delta(x)=\Delta_{s} e^{i \vartheta(x)}$ onto the effective forward scattering potential $V(x)=\partial_{x} \vartheta(x) / 2$.

\footnotetext{
${ }^{5}$ The prefactor can be expressed in terms of the Euler constant $\gamma$, such that $\Delta_{s} \xi(T)=s T_{c}^{\mathrm{MF}} / 2 e^{\gamma} T$.
} 


\subsubsection{Density of states and inverse localization length}

The DOS and the inverse localization length for the remaining problem involving only a constant gap parameter and forward scattering described by Gaussian white noise were already calculated in the previous chapter. With the exception of the constant shift in the inverse localization length, the results are identical with those for the incommensurate case without forward scattering and $\langle\Delta(x)\rangle=\Delta_{0} \neq 0$. Substituting in Eqs. (3.71) and (3.73) $\Delta_{0}$ by $\Delta_{s}, \tilde{D}$ by $1 / 4 \xi(T)$ and setting $D=0$ (since there is only forward scattering) which implies a completely different interpretation of the resulting equations, we get for $\Gamma(\omega)=\ell^{-1}(\omega)-i \pi \mathcal{N}(\omega)$

$$
\Gamma(\omega)=-i \omega+\Delta_{s} \frac{I_{1-i 4 \omega \xi}\left(4 \Delta_{s} \xi\right)}{I_{-i 4 \omega \xi}\left(4 \Delta_{s} \xi\right)},
$$

or, equivalently,

$$
\Gamma(\omega)=\Delta_{s} \frac{I_{-i 4 \omega \xi}^{\prime}\left(4 \Delta_{s} \xi\right)}{I_{-i 4 \omega \xi}\left(4 \Delta_{s} \xi\right)} .
$$

It follows from Eq. (3.75) that the integrated DOS is given by

$$
\mathcal{N}(\omega)=\rho_{0} \frac{\sinh (4 \pi \omega \xi)}{4 \pi \xi} \frac{1}{\left|I_{i 4 \omega \xi}\left(4 \Delta_{s} \xi\right)\right|^{2}}
$$

Plots of the DOS and the inverse localization length for characteristic values of $\Delta_{S} \xi$ are shown in Fig. 4.6. At the Fermi energy, the DOS simplifies to

$$
\rho(0)=\frac{\rho_{0}}{\left[I_{0}\left(4 \Delta_{s} \xi\right)\right]^{2}},
$$

such that, as the temperature is lowered and the correlation length grows, the DOS at the Fermi energy vanishes exponentially,

$$
\rho(0) \sim 8 \pi \rho_{0} \Delta_{s} \xi \exp \left(-8 \Delta_{s} \xi\right), \quad 4 \Delta_{s} \xi \gg 1 .
$$

This result is in contrast to the power-law behavior of the DOS as predicted by Gaussian statistics. A plot of the DOS at the Fermi energy is shown in Fig. 4.7 as a function of $1 / \Delta_{s} \xi$. For a comparison, we have also plotted $\rho(0)$ for Gaussian statistics and the result found in the Born approximation, which at low temperatures can only poorly describe the quantitative behavior of the pseudogap. For $T=T_{c}^{\mathrm{MF}} / 4$ which corresponds to $\Delta_{s} \xi \approx 2.0$, we find that the $\operatorname{DOS} \rho(0)$ for phase fluctuations only is less than $10^{-5} \rho_{0}$ while the Born approximation and the numerical exact result for Gaussian statistics suggest a value of order $\rho_{0} / 4$.

As can also be seen in Fig. 4.7 there is no pseudogap for $\Delta_{s} \xi \lesssim 0.1$. Note, however, that these correlation lengths correspond to temperatures of order $T_{c}^{\mathrm{MF}}$, where the DOS 

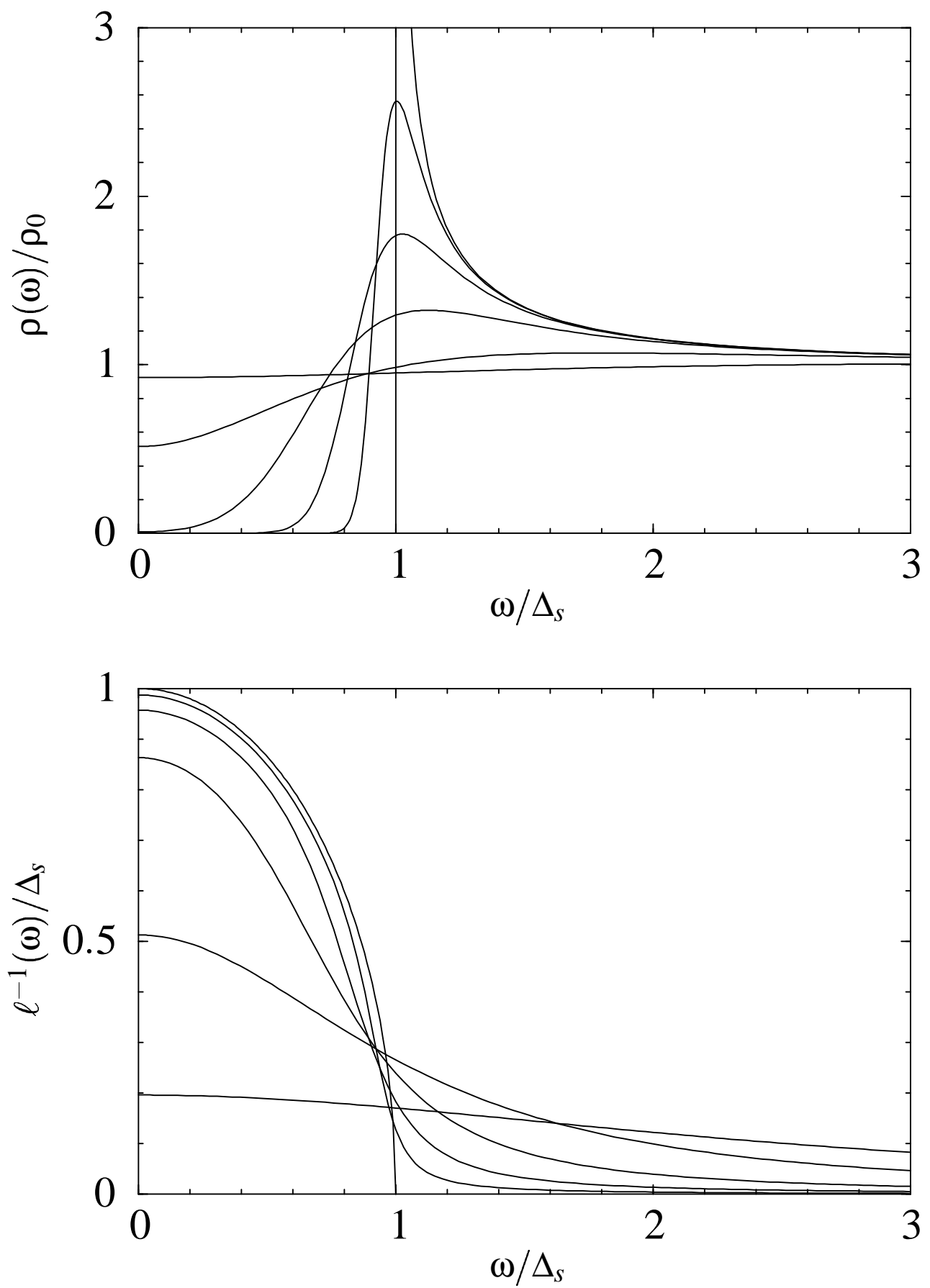

Figure 4.6: Plot of the DOS $\rho(\omega)$ and the inverse localization length $\ell^{-1}(\omega)$ for phase fluctuations only and $\Delta_{s} \xi=0.1,0.3,1.0,3.0,10.0$ and $\infty$. As $\Delta_{s} \xi$ increases, the pseudogap in the DOS becomes deeper and the inverse localization length increases for small frequencies. 


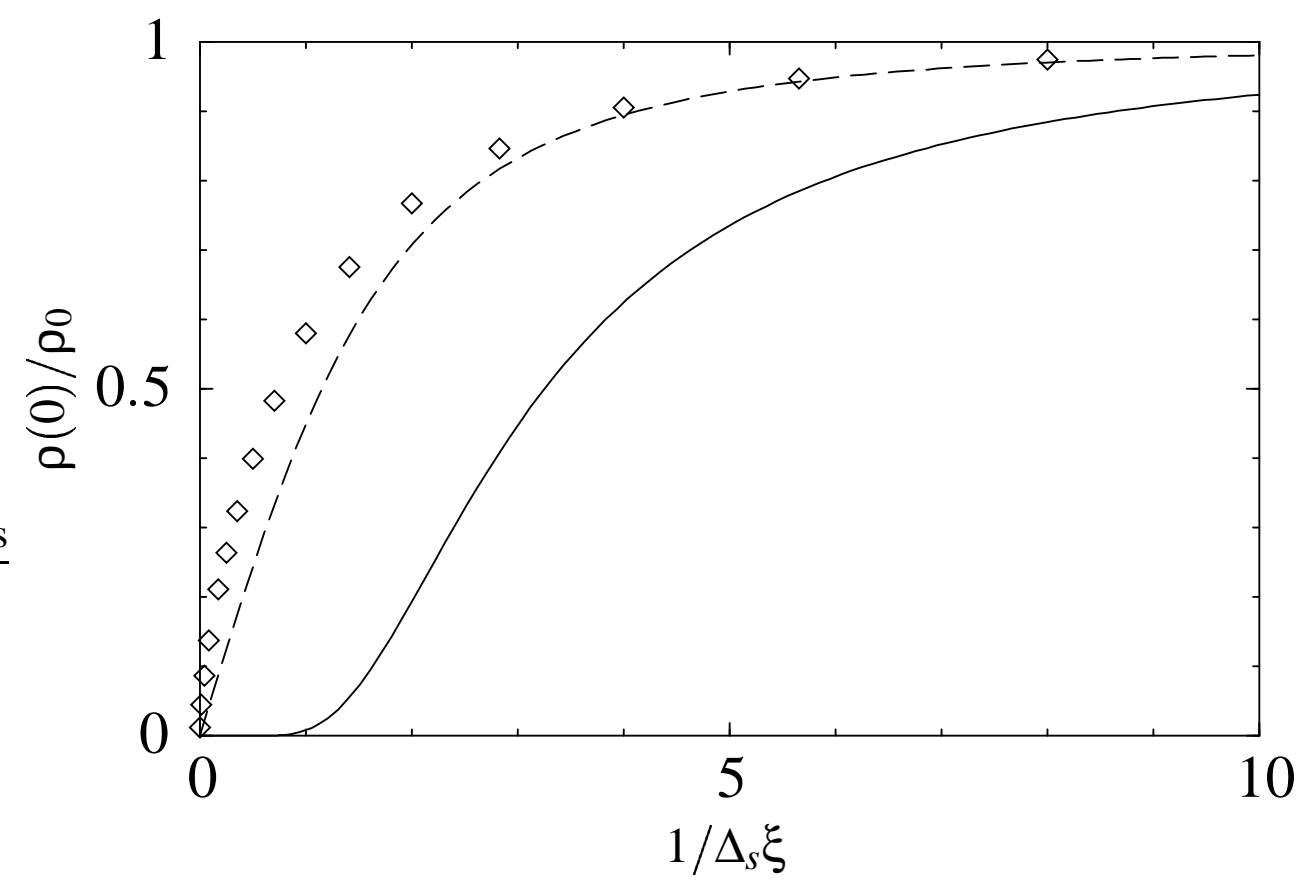

Figure 4.7: Plot of the DOS $\rho(0) / \rho_{0}$ as a function of $1 / \Delta_{s} \xi$. The solid line gives the DOS for phase fluctuations only while the dashed line is the result found in the leading order Born approximation [see Eq. 2.27] and diamonds give the DOS evaluated for Gaussian statistics [see Section 4.2]

has to be described by amplitude fluctuations. Nevertheless, using only phase fluctuations for all temperatures $T$ gives a good qualitative description of the DOS also in the temperature region where amplitude fluctuations are important.

For temperatures well below the mean-field temperature $T_{c}^{\mathrm{MF}}$, where our theory becomes quite accurate, we have, according to Eq. [4.40,,$\Delta_{s} \xi \gg 1$, such that the Bessel function

$$
I_{i v}(v z)=e^{-\pi v / 2} J_{i v}(i v z)
$$

may be approximated by an Airy function: Using Eq. (9.3.35) from Ref. [4] (A\&S),

$$
J_{v}(v z) \sim\left(\frac{4 \zeta}{1-z^{2}}\right)^{1 / 4} \frac{\mathrm{Ai}\left(v^{2 / 3} \zeta\right)}{v^{1 / 3}}
$$

with

$$
\begin{aligned}
\frac{2}{3} \zeta^{3 / 2} & =\ln \frac{1+\sqrt{1-z^{2}}}{z}-\sqrt{1-z^{2}}, \quad z<1, \\
\frac{2}{3}(-\zeta)^{3 / 2} & =\sqrt{z^{2}-1}-\arccos \left(\frac{1}{z}\right), \quad z>1,
\end{aligned}
$$


whose leading terms in the Taylor expansion are given by

$$
\zeta=2^{\frac{1}{3}}\left((1-z)+\frac{3}{10}(1-z)^{2}+\frac{32}{175}(1-z)^{3}\right)+O(1-z)^{4}
$$

we find

$$
\mathcal{N}(\omega)=\rho_{0}\left(\frac{\omega^{2}-\Delta_{s}^{2}}{\zeta}\right)^{1 / 2} \frac{1-e^{-4 \pi \omega \xi}}{2 \pi(4 \omega \xi)^{1 / 3}} \frac{1}{\left|\operatorname{Ai}\left((i 4 \omega \xi)^{2 / 3} \zeta\right)\right|^{2}} .
$$

Here, $\zeta$ is given by Eqs. (4.50) and (4.51) with $z=\Delta_{s} / \omega$. Note that Eq. (4.53) is valid for arbitrary $\omega$ as long as $\Delta_{s} \xi \gg 1$. The case of large correlation lengths can be surveyed further as follows: Expanding Eq. (4.53) for $\omega$ around $\Delta_{s}$, we find to leading order in $1 / 4 \Delta_{s} \xi$ a maximum of the $\operatorname{DOS} \rho(\omega)$ at $\Delta_{s}$, described by the inverted parabola

$$
\rho(\omega) \sim \rho_{0}\left[a\left(4 \Delta_{s} \xi\right)^{1 / 3}-b\left(4 \Delta_{s} \xi\right)^{5 / 3}\left(\frac{\omega}{\Delta_{s}}-1\right)^{2}\right]
$$

with

$$
\begin{aligned}
& a=\frac{1}{2^{4 / 3} \pi} \frac{c_{2}}{c_{1}^{3}} \approx 0.731, \\
& b=\frac{1}{2^{2 / 3} \pi} \frac{1}{c_{1}^{2}}\left[3\left(\frac{c_{2}}{c_{1}}\right)^{3}-1\right] \approx 0.258,
\end{aligned}
$$

where $c_{1}=\operatorname{Ai}(0)=3^{-2 / 3} / \Gamma(2 / 3) \approx 0.355$ and $c_{2}=-\mathrm{Ai}^{\prime}(0)=3^{-1 / 3} / \Gamma(1 / 3) \approx$ 0.259. Note that Eq. (4.54) implies that the maximum of the DOS diverges as $\left(4 \Delta_{s} \xi\right)^{1 / 3} \propto T^{-1 / 3}$.

Away from $\omega=\Delta_{s}$, the argument of the Airy function is large such that $\operatorname{Ai}\left((i 4 \omega \xi)^{2 / 3} \zeta\right)$ may be expanded in an asymptotic series: If we use Eq. (10.4.59) from $A \& S$,

$$
\operatorname{Ai}(z) \sim(1 / 2) \pi^{-1 / 2} z^{-1 / 4} \exp \left(-2 z^{3 / 2} / 3\right), \quad(|\arg |<\pi),
$$

we find for $\omega>\Delta_{s}$

$$
\rho(\omega) \sim \rho_{0}\left(1+\frac{\Delta_{s}^{2}}{4 \omega^{2}}\right), \quad 4 \Delta_{s} \xi\left(\frac{\omega}{\Delta_{s}}-1\right)^{3 / 2} \gg 1,
$$

which is independent of $\Delta_{s} \xi$ and agrees with the mean-field result.

For $\omega<\Delta_{s}$, we can use Eq. (10.4.60) from $A \& S$,

$$
\operatorname{Ai}(-z) \sim \pi^{-1 / 2} z^{-1 / 4} \sin \left(\frac{2}{3} z^{3 / 2}+\frac{\pi}{4}\right), \quad(|\arg |<2 \pi / 3)
$$


to find for a quantitative description of the pseudogap

$$
\begin{aligned}
\rho(\omega) \sim 8 \rho_{0} \sqrt{\Delta_{s}^{2}-\omega^{2}} \xi \arccos \left(\omega / \Delta_{s}\right)[1+\exp (-8 \pi \omega \xi)] \\
\times \exp \left(-8\left[\sqrt{\Delta_{s}^{2}-\omega^{2}} \xi-\omega \xi \arccos \left(\omega / \Delta_{s}\right)\right]\right) \\
4 \Delta_{s} \xi\left(1-\frac{\omega}{\Delta_{s}}\right)^{3 / 2} \gg 1 .
\end{aligned}
$$

If $\omega \ll \Delta_{s}$ and $4 \omega^{2} \xi / \Delta_{s} \ll 1$, this result simplifies to

$$
\rho(0) \sim 8 \pi \rho_{0} \Delta_{s} \xi \cosh (4 \pi \omega \xi) \exp \left(-8 \Delta_{s} \xi\right),
$$

which at $\omega=0$ agrees with Eq. (4.47).

\subsubsection{Pauli paramagnetic susceptibility}

The Pauli paramagnetic susceptibility is defined as the contribution of the conduction electrons to the susceptibility and can be written in terms of the DOS. A magnetic field $H$ shifts the energy levels of the electrons by an amount $\pm \mu_{B} H$, where $\mu_{B}$ is the Bohr magneton and the sign depends on the spin orientation of the electron with respect to the field. The resulting different occupation of spin-up and spin-down states leads to a magnetization density $M(T)$ which for small magnetic fields is linear in $H$. An elementary calculation of the susceptibility $\chi(T) \equiv d M(T) / d H$, which can be found in the textbook by Ashcroft and Mermin [6], gives

$$
\chi(T)=\mu_{B}^{2} \int_{-\infty}^{\infty} d \omega 2 \rho^{\prime}(\omega) f(\omega) .
$$

Here, $f(\omega)=1 /(\exp (\omega / T)+1)$ is the Fermi function, $\rho^{\prime}(\omega)$ is the derivative of the DOS with respect to frequency, and the factor of 2 is due to the two spin directions. Integrating by parts, we find

$$
\chi(T)=\mu_{B}^{2} \int_{-\infty}^{\infty} d \omega 2 \rho(\omega)\left(-\frac{d f(\omega)}{d \omega}\right) .
$$

At $T=0$, we have $f(\omega)=\theta(-\omega)$, such that $-d f(\omega) / d \omega=\delta(\omega)$, and

$$
\chi(T=0)=2 \mu_{B}^{2} \rho(0) .
$$

At zero temperature, the Pauli paramagnetic susceptibility is proportional to the DOS at the Fermi energy. In usual metals, $\rho(0)$ is finite at $T=0$, and Eq. (4.64) holds up to $T \approx 10000 K$ (see Ref. [6]). In the case of a pseudogap or a singularity at the Fermi energy, however, we have to go back to Eq. 4.63), which can also be written as

$$
\chi(T)=\frac{\mu_{B}^{2}}{T} \int_{0}^{\infty} d \omega \rho(\omega) \frac{1}{\cosh ^{2}(\omega / 2 T)}
$$


Placing the asymptotic expression (4.60) with $\xi(T)=\rho_{s} / T$ into this equation, we are left with

$$
\begin{gathered}
\chi(T) / \chi_{0} \sim \frac{16 \rho_{s}}{T^{2}} \int_{0}^{\infty} d \omega \sqrt{\Delta_{s}^{2}-\omega^{2}} \arccos \left(\frac{\omega}{\Delta_{s}}\right) \frac{1+\exp \left[-8 \pi \rho_{s} \omega / T\right]}{(1+\exp [-2 \omega / T])^{2}} \\
\quad \times \exp \left(-\frac{\Delta_{s}}{T}\left[\frac{\omega}{\Delta_{s}}\left(1-8 \rho_{s} \arccos \left(\frac{\omega}{\Delta_{s}}\right)\right)+8 \rho_{s} \sqrt{1-\left(\omega / \Delta_{s}\right)^{2}}\right]\right),
\end{gathered}
$$

where

$$
\chi_{0}=2 \mu_{B}^{2} \rho_{0},
$$

For $\rho_{s}>\rho_{0} / 4$ (and $T \lll T_{c}^{\mathrm{MF}}$ ), the integrand is sharply peaked at $\omega=\cos \left(1 / 8 \rho_{s}\right) \Delta_{s}$, such that the integral may be evaluated by a saddle point integration, resulting in

$$
\begin{aligned}
& \frac{\chi(T)}{\chi_{0}} \sim \sqrt{\frac{\pi}{\rho_{s}}}\left(\frac{\sin \left(1 / 8 \rho_{s}\right) \Delta_{s}}{T}\right)^{3 / 2} \exp \left(-\frac{8 \rho_{s} \sin \left(1 / 8 \rho_{s}\right) \Delta_{s}}{T}\right), \\
& T \ll 4 \rho_{s} \Delta_{s}\left(1-\cos \left(1 / 8 \rho_{s}\right)\right)^{3 / 2}
\end{aligned}
$$

Note, that the temperature restriction is necessary for the asymptotic expansion of the DOS to be valid. Although the susceptibility $\chi(T)$ vanishes exponentially, the exponent $8 \rho_{s} \sin \left(1 / 8 \rho_{s}\right) \Delta_{s} / T$ is smaller than the exponent $8 \rho_{s} \Delta_{s} / T$, which governs the DOS at the Fermi energy. However, as $\rho_{s}$ approaches $\rho_{0} / 4$, the two exponents become identical, such that for $\rho_{s} \leq \rho_{0} / 4$ the DOS and the susceptibility have the same exponential dependence on $T$.

A numerical evaluation of the susceptibility $\chi(T) / \chi_{0}$ for $\xi(T)=\rho_{s}(T) T$ given by Eq. (1.122) and $\Delta_{S}(T)$ determined by the BCS gap equation (1.83) is shown in Fig. 4.8. For a comparison, we have also plotted the DOS $\rho(0) / \rho_{0}$ as a function of temperature. The two are not identical because for small temperatures (and $\rho_{s}=\rho_{0}$ ), the major contribution to the integral in Eq. (4.65) comes from the frequency region just below $\Delta_{s}$. In Fig. 4.8, we also show susceptibility data taken from Ref. [45] for incommensurate quasi one-dimensional conductors which undergo a Pererls transition.

It is quite surprising that our plot of the susceptibility is very similar to the plot obtained by Lee, Rice and Anderson [57] which perfectly fits experimental data [37, 44, 45]. However, to explain experimental data, Lee, Rice and Anderson [57] had to base their calculations on a real order parameter with a correlation length which for low temperatures increases exponentially as the temperature is lowered. Only the exponentially increasing correlation length of a real order parameter could lead to an exponentially decreasing susceptibility and the prediction of $T_{c}^{3 \mathrm{D}} \approx T_{c}^{\mathrm{MF}} / 4$. Here, we have shown that these predictions should also hold for a complex order parameter with a correlation length which increases as $1 / T$. Since most Peierls chains are incommensurate and the susceptibility of many incommensurate Peierls chains has been compared with the theory by Lee, Rice and Anderson [57], our results are of major experimental relevance. For a comparison between theory and experiment, it should be recalled that we have only used a strictly one-dimensional model with phase fluctuations only. At higher temperatures, one should also include amplitude fluctuations. 


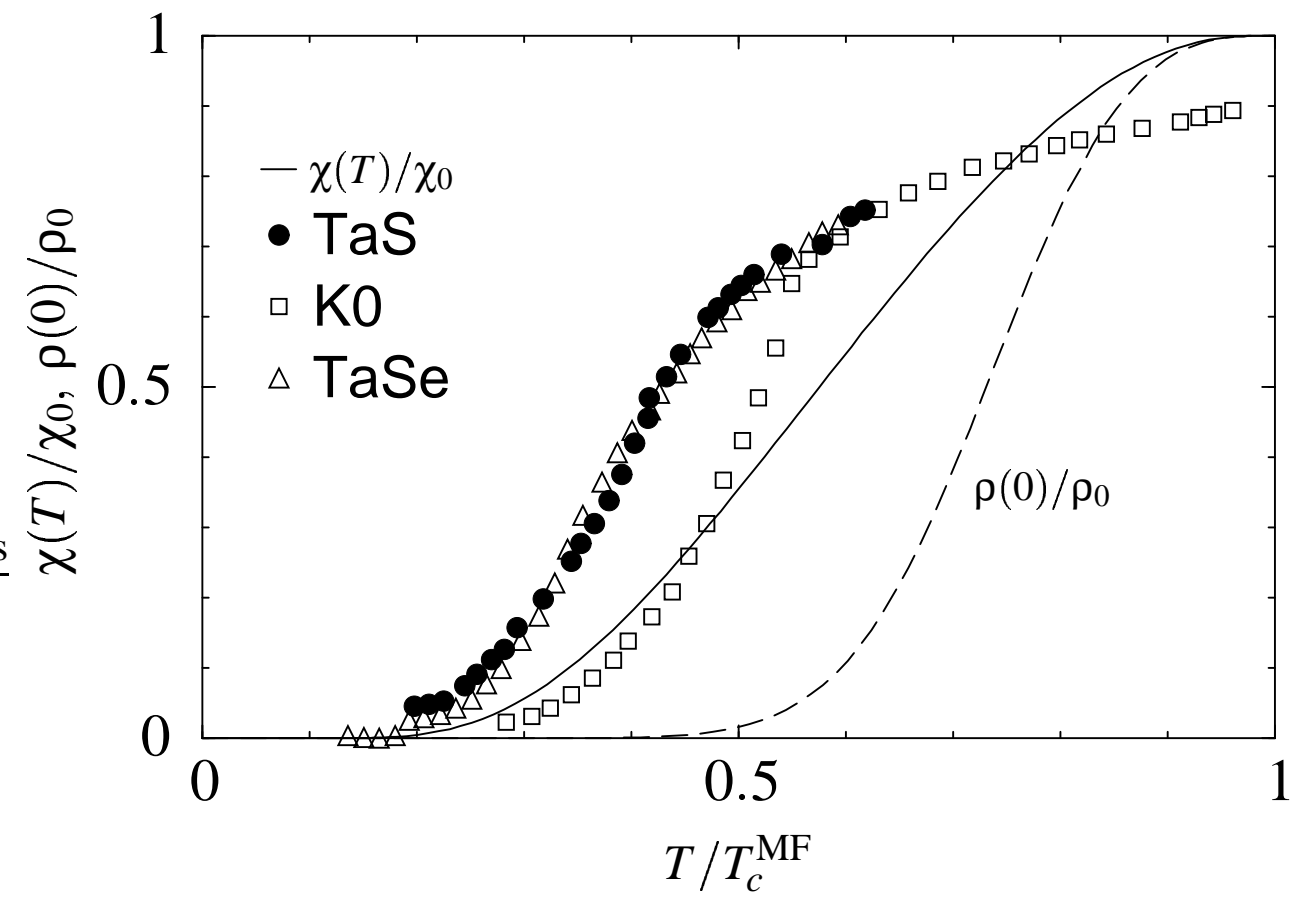

Figure 4.8: Plot of the susceptibility $\chi(T)$ calculated for $\xi(T)=\rho_{s}(T) / T$ [with $\rho_{s}(T)$ given by Eq. [1.122)] and $\Delta_{s}(T)$ determined by the minimum of the Ginzburg-Landau functional. For a comparison, we also show as the dashed line the DOS at the Fermi energy, $\rho(0)$.

\subsubsection{Thermodynamic quantities}

The DOS encapsulates the whole thermodynamics. Let us first consider the electronic free energy, $F_{\mathrm{el}}(T)$ with respect to the gapped state with $\rho_{\infty}(\omega)=\rho_{0} \theta\left(\omega^{2}-\right.$ $\left.\Delta_{S}^{2}\right)|\omega| /\left(\omega^{2}-\Delta_{S}^{2}\right)$ :

$$
F_{\mathrm{el}}(T)-F_{\mathrm{el}}^{\xi=\infty}(T)=-\frac{s L}{\beta} \int_{-\infty}^{\infty} d \omega\left[\rho(\omega)-\rho_{\infty}(\omega)\right] \ln \left(1+e^{-\beta \omega}\right) .
$$

Partial integration leads to

$$
\begin{aligned}
F_{\mathrm{el}}(T)-F_{\mathrm{el}}^{\xi=\infty}(T) & =-s L \int d \omega\left[\mathcal{N}(\omega)-\mathcal{N}_{\infty}(\omega)\right] \frac{1}{e^{\beta \omega}+1} \\
& =s \rho_{0} L \operatorname{Im} \int d \omega\left[\Gamma(\omega)-\Gamma_{\infty}(\omega)\right] \frac{1}{e^{\beta \omega}+1}
\end{aligned}
$$

where $\Gamma(\omega)=\ell^{-1}(\omega)-i \pi \mathcal{N}(\omega)$ is the space-averaged integrated retarded Green function at coinciding space points already considered before and $\Gamma_{\infty}(\omega)=$ $\sqrt{\Delta_{s}^{2}-(\omega+i 0)^{2}}$, where the square root has to be taken such that $\Gamma_{\infty}(\omega) \rightarrow-i \omega$ for 
$\omega \rightarrow \infty$. Since $\Gamma(\omega)$ is analytic in the upper half plane, the integral may be done by closing the integral in the upper half plane and using the residue theorem. We find

$$
F_{\mathrm{el}}(T)-F_{\mathrm{el}}^{\xi=\infty}(T)=-s \rho_{0} L \frac{2 \pi}{\beta} \sum_{\tilde{\omega}_{n}>0}\left[\operatorname{Re} \Gamma\left(i \tilde{\omega}_{n}\right)-\tilde{\omega}_{n}\right]
$$

For the FGM with phase fluctuations only, we obtain by placing Eq. (4.44) into this equation

$$
F_{\mathrm{el}}(T)-F_{\mathrm{el}}^{\xi=\infty}(T)=s \rho_{0} L \Delta_{s} \frac{2 \pi}{\beta} \sum_{\tilde{\omega}_{n}>0}\left[\sqrt{1+\left(\frac{\tilde{\omega}_{n}}{\Delta_{s}}\right)^{2}}-\frac{I_{4 \tilde{\omega}_{n} \xi}^{\prime}\left(4 \Delta_{s} \xi\right)}{I_{4 \tilde{\omega}_{n} \xi}\left(4 \Delta_{s} \xi\right)}\right] .
$$

Up to an irrelevant additive constant, $F_{\mathrm{el}}^{\xi=\infty}(T)$ is given by

$$
F_{\mathrm{el}}^{\xi=\infty}(T)=-s \rho_{0} L 2 \Delta_{s}^{2} \int_{1}^{\infty} d u \sqrt{u^{2}-1} \frac{1}{e^{\Delta_{s} u / T}+1},
$$

which for small temperatures is exponentially small:

$$
F_{\mathrm{el}}^{\xi=\infty}(T) \sim-s \rho_{0} L \sqrt{2 \pi} \Delta_{s}^{2} e^{-\Delta_{s} / T}, \quad T \ll \Delta_{s} .
$$

While in the general case we have to add Eqs. (4.72) and (4.73) to get the free energy $F_{\text {el }}(T)$, for low temperatures we can neglect the exponentially small contribution given by Eq. (4.74), such that $F_{\mathrm{el}}(T)$ is determined by the right-hand side of Eq. (4.72).

For $T \ll T_{c}^{\mathrm{MF}}$, the dimensionless correlation length $\Delta_{s} \xi$ is large, and a uniform asymptotic expansion of $I_{v}(v z)$ and $I_{v}^{\prime}(v z)$ [see A\&S, Eqs. (9.7.7) and (9.7.9)] can be used to find for the leading terms of the free energy

$$
F(T) \sim s \rho_{0} L \Delta_{s}^{2}\left[\frac{\pi}{4} \frac{1}{4 \Delta_{s} \xi}-\frac{1}{12} \frac{1}{\left(4 \Delta_{s} \xi\right)^{2}}\right]
$$

\section{Electronic specific heat}

An experimentally accessible thermodynamic quantity is the electronic specific heat which can be expressed in terms of the free energy as

$$
C_{\mathrm{el}}(T)=-T \frac{d^{2} F_{\mathrm{el}}(T)}{d T^{2}} .
$$

The low-temperature behavior of $C_{\mathrm{el}}(T)$ can be obtained from Eq. (4.75): Using $\xi(T)=s \rho_{s}(0) / 2 T$, it directly follows

$$
C_{\mathrm{el}}(T) \sim \frac{1}{8}\left(\frac{\rho_{0}}{s \rho_{s}(0)}\right)^{2} C_{\mathrm{el}}^{0}(T)
$$


where the specific heat of free electrons is given by

$$
C_{\mathrm{el}}^{0}(T)=s \frac{\pi^{2}}{3} \rho_{0} L T .
$$

Although the DOS exhibits a pseudogap and vanishes exponentially near the Fermi energy as the temperature is lowered, the electronic specific heat $C_{\mathrm{el}}(T)$ vanishes only linearly in $T$, as for free electrons.

We conclude this chapter with a summary of the central results of the FGM valid at low temperatures where phase fluctuations dominate in Table 4.1

Table 4.1: Asymptotic low temperature results for the FGM describing electrons with spin. Note that we have reintroduced the Fermi velocity $v_{F}$ and note also that the meanfield critical temperature $T_{c}^{\mathrm{MF}}$ serves as the only energy scale. For generalizations of the formulas see the text.

\begin{tabular}{||ll|l}
\hline superfluid density & $\rho_{s}(T)$ & $\sim \rho_{0}=\frac{1}{\pi v_{F}}$ \\
correlation length & $\xi(T)$ & $\sim \frac{v_{F}}{\pi T}$ \\
density of states & $\frac{\rho(0)}{\rho_{0}}$ & $\sim \frac{14.1 T_{c}^{\mathrm{MF}}}{T} \exp \left(-\frac{4.49 T_{c}^{\mathrm{MF}}}{T}\right)$ \\
inverse localization length & $\ell^{-1}(0)$ & $\sim \frac{1.76 T_{c}^{\mathrm{MF}}}{v_{F}}$ \\
susceptibility & $\frac{\chi(T)}{\chi_{0}}$ & $\sim 1.74\left(\frac{T_{c}^{\mathrm{MF}}}{T}\right)^{3 / 2} \exp \left(-\frac{1.72 T_{c}^{\mathrm{MF}}}{T}\right)$ \\
electronic specific heat & $\frac{C_{\mathrm{el}}(T)}{C_{\mathrm{el}}^{0}(T)}$ & $\sim \frac{1}{32}$
\end{tabular}





\section{Conclusion}

In this work, we have discussed the density of states (DOS) of the fluctuating gap model (FGM) and related quantities like the inverse localization length, the Pauli paramagnetic susceptibility and the low-temperature specific heat. We introduced the FGM as an effective low-energy model describing the electronic properties of Peierls chains and emphasized the fact that the FGM also finds its applications in other physical contexts: Spin chains can be mapped by a Jordan-Wigner transformation onto the FGM and in order to explain the pseudogap-phenomenon in underdoped cuprates above a phase transition, higher-dimensional generalizations of the FGM have been used.

With the rediscovery of the FGM in the context of high-temperature superconductivity, a previously unnoticed subtle error surfaced in Sadovskii's widely used Green function of the FGM, calculated for Gaussian statistics with finite correlation lengths. This error re-opened the whole problem.

After setting up a non-perturbative theory which, in principle, allows to express the one-particle Green function as a functional of an arbitrary given realization of the disorder, we derived a simple equation of motion whose solution determines the DOS and the inverse localization length. Starting from this equation, we could rederive all known results for the FGM in the white noise limit.

Considering the equation of motion governed by the phase which determines the DOS, we argued that the Dyson singularity found in the white noise limit for commensurate Peierls chains should not be an artifact of the white noise limit, but should be present for any finite correlation length in contradiction to Sadovskii's solution. Our following numerical calculation of the DOS and inverse localization length confirmed this prediction and showed also that for large correlation lengths, the Dyson singularity only overshadows a pseudogap. Although Sadovskii's algorithm misses this singularity, his solutions for the incommensurate case where there are no singularities in the DOS give a fairly good approximation to the exact result.

In the pseudogap-regime below the mean-field critical temperature, fluctuations of the order parameter cannot be described by Gaussian statistics. Instead, as the temperature is lowered, amplitude fluctuations get gradually frozen out, and the amplitude takes on a value given by the minimum of the Ginzburg-Landau functional and only long-wavelength gapless phase fluctuations survive. Using a gauge transformation to map the phase fluctuations of the order parameter onto an effective forward scatter- 
ing potential, we could even find an exact solution for the FGM involving only phase fluctuations which should be valid in the low temperature regime. We found that the low-temperature specific heat is linear in $T$ and that both the DOS at the Fermi energy and the Pauli paramagnetic susceptibility vanish exponentially as the temperature $T$ is lowered, the ratio of the former to the latter also vanishing exponentially. The Pauli paramagnetic susceptibility has been measured in various experiments and is in good agreement with our results.

Having discussed quantities related to the DOS, one would also like to calculate quantities like the spectral function. This has been done for a special non-Gaussian probability distribution involving amplitude and phase fluctuations in Ref. [13], but accurate results for realistic probability distributions (e.g. for phase fluctuations only) are not known yet. 


\section{Appendix A}

\section{Gradient expansion of the free energy}

In this appendix we present an efficient algorithm for obtaining the gradient expansion of the local density of states and the free energy functional of the fluctuating gap model (FGM). The algorithm is based on the linear pseudo-Schrödinger equation derived in Chapter 2 The three-component wave-function $\vec{\psi}(x)$ satisfies a non-linear constraint and may be found by a simple iterative procedure. Since one component of $\vec{\psi}(x)$ is directly related to the local density of states (DOS), we obtain a simple iterative algorithm to develop the gradient expansion of the local DOS and therefore also the free energy fuctional. A generalization of the results to a three-dimensional clean superconductor is given in Ref. [10].

\section{Introduction}

The phenomenological Ginzburg-Landau theory has proven to be a powerful tool in the theory of superconductivity. Starting from the Gorkov equations of superconductivity, it is also possible to derive the Ginzburg-Landau functional microscopically. For the Ginzburg-Landau expansion to be valid, the order parameter field has to be small. In a mean-field picture this is the case near a phase transition. However, away from criticality or if fluctuations are large, one needs to include terms of higher order. In the case of superconductivity, the extension towards arbitrary temperatures has been done in the sixties by Werthamer [94, 95] and Tewordt [88, 89], who expanded the free energy in terms of gradients of the order parameter. Unfortunately, this direct expansion of the free energy in powers of gradients of $\Delta(x)$ is quite laborious and rather difficult to verify.

Recently Kosztin, Kos, Stone and Leggett [53], and Kos and Stone [52] (KKSL) developed new and more efficient algorithms to obtain the gradient expansion of the free energy $F\left\{\Delta, \Delta^{*}\right\}$ of a clean superconductor and found a discrepancy with the expression published by Tewordt. In Ref. [10] we confirmed the result derived by KKSL by reducing the three-dimensional problem in a semiclassical approximation to the problem of finding the gradient expansion of the one-dimensional FGM, and determining its gradient expansion by an iterative solution of the pseudo-Schrödinger 
equation (2.133) derived in Chapter 2 Since, as was shown in Chapter 2 , our pseudoSchrödinger equation is in fact equivalent to the Eilenberger equation of superconductivity which was used by KKSL, our determination of the gradient expansion of the free energy is related to the one used by KKSL. The essential difference, however, is the implementation of our non-linear constraint, which turns out to be quite useful. In the following we will present our derivation of the gradient expansion of the local DOS (which was first published in [10]) and the free energy functional of the one-dimensional FGM. The semiclassical generalization towards higher dimensions is given in [10].

In Chapter 2 we have shown that the local DOS can be obtained from the second component of the three-component vector $\vec{\psi}(x)$, which satisfies the linear pseudoSchrödinger equation [see Eq. (2.133)]

$$
-\partial_{x} \vec{\psi}(x)=H(x) \vec{\psi}(x),
$$

with the pseudo-Hamiltonian given by

$$
H(x)=2 i \omega J_{3}+\Delta(x) J_{-}+\Delta^{*}(x) J_{+} .
$$

The $J_{i}$ are spin $J=1$ operators in the representation

$$
J_{3}=\left(\begin{array}{ccc}
1 & 0 & 0 \\
0 & 0 & 0 \\
0 & 0 & -1
\end{array}\right), J_{+}=\sqrt{2}\left(\begin{array}{ccc}
0 & 1 & 0 \\
0 & 0 & 1 \\
0 & 0 & 0
\end{array}\right), J_{-}=\sqrt{2}\left(\begin{array}{ccc}
0 & 0 & 0 \\
1 & 0 & 0 \\
0 & 1 & 0
\end{array}\right)
$$

Recall that $\vec{\psi}(x)$ has to satisfy the constraint

$$
\tilde{\psi}^{T}(x) \vec{\psi}(x)=1
$$

where

$$
\tilde{\psi}^{T}(x)=\left(-\psi_{3}(x), \psi_{2}(x),-\psi_{1}(x)\right) .
$$

In terms of $\vec{\psi}(x)$ the local DOS (per spin direction) is given by

$$
\rho(\omega ; x)=\rho_{0} \operatorname{Re} \psi_{2}(x) .
$$

\section{Iterative algorithm and gradient expansion}

The gradient expansion of the local DOS is directly obtained from the second component of the gradient expansion of $\vec{\psi}(x)$. For convenience, we develop the gradient expansion of $\vec{\psi}(x)$ for imaginary frequencies $\omega=i E$, because then our pseudoHamiltonian (A.2) is Hermitian and left and right eigenvectors are identical. Suppose we expand the solution of Eq. A.1) in the form

$$
\vec{\psi}(x)=\sum_{n=0}^{\infty} \vec{\psi}_{n}(x)
$$


where by definition $\vec{\psi}_{n}(x)$ involves $n$ derivatives with respect to $x$. Obviously

$$
H(x) \vec{\psi}_{0}(x)=0,
$$

i.e. $\vec{\psi}_{0}(x)$ must be an eigenvector of $H(x)$ with eigenvalue zero. The existence of such an eigenvector follows trivially from the fact that our pseudo-Hamiltonian (A.2) can be interpreted as the Zeeman-Hamiltonian of a $J=1$ quantum spin in an external magnetic field. Note that Eq. A.8 determines $\vec{\psi}_{0}(x)$ only up to an overall multiplicative factor, which is fixed by requiring that the components of $\vec{\psi}_{0}(x)$ satisfy the constraint (A.4). This yields (with $\omega=i E$ )

$$
\vec{\psi}_{0}(x)=\frac{1}{\sqrt{E^{2}+|\Delta(x)|^{2}}}\left(\begin{array}{c}
\frac{\Delta^{*}(x)}{\sqrt{2}} \\
E \\
-\frac{\Delta(x)}{\sqrt{2}}
\end{array}\right) .
$$

For the higher order terms we obtain the simple recursion relation

$$
\partial_{x} \vec{\psi}_{n}(x)=-H(x) \vec{\psi}_{n+1}(x), n=0,1, \ldots
$$

Because one of the eigenvalues of $H(x)$ vanishes, the inverse of $H(x)$ does not exist, so that we cannot simply solve Eq. (A.10) by multiplying both sides by $H^{-1}(x)$. As a consequence, Eq. A.10) determines $\vec{\psi}_{n+1}(x)$ only up to a vector proportional to $\vec{\psi}_{0}(x)$,

$$
\vec{\psi}_{n+1}(x)=-H_{\perp}^{-1}(x) \partial_{x} \vec{\psi}_{n}(x)+c_{n+1}(x) \vec{\psi}_{0}(x),
$$

where $H_{\perp}^{-1}(x)$ is the inverse of $H(x)$ in the subspace orthogonal to $\vec{\psi}_{0}(x)$. Using the fact that the two non-vanishing eigenvalues of $H(x)$ are given by $\pm 2\left[E^{2}+|\Delta(x)|^{2}\right]^{1 / 2}$, we find

$$
H_{\perp}^{-1}(x)=\frac{H(x)}{4\left[E^{2}+|\Delta(x)|^{2}\right]},
$$

i.e. $H_{\perp}^{-1}(x)$ is proportional to $H(x)$. To fix the constant $c_{n+1}(x)$ in Eq. A.11, we require that the components of $\sum_{i=0}^{n+1} \vec{\psi}_{i}(x)$ satisfy the constraint A.4. This implies

$$
c_{n+1}(x)=-\frac{1}{2} \sum_{i=1}^{n} \tilde{\psi}_{i}^{T}(x) \vec{\psi}_{n+1-i}(x),
$$

where the vector $\tilde{\psi}_{i}(x)$ is obtained from $\vec{\psi}_{i}(x)$ by exchanging the first and third components and multiplying them by -1 , see Eq. (A.5). For odd $n$ we can show that $c_{n}(x)=0$. We thus obtain an explicit and very compact recursive algorithm for calculating the gradient expansion of the local DOS. To zeroth order the vector $\vec{\psi}(x)$ is given by Eq. A.9. This corresponds to the adiabatic approximation of elementary quantum mechanics. The step $n \rightarrow n+1$ is summarized as follows:

- $\vec{\psi}_{i}$ given for $i=0, \ldots, n$

- $\vec{\psi}_{n+1}=-H_{\perp}^{-1} \partial_{x} \vec{\psi}_{n}-\frac{\vec{\psi}_{0}}{2} \sum_{i=1}^{n} \tilde{\psi}_{i}^{T} \vec{\psi}_{n+1-i}$. 
It is easy to implement this iterative algorithm on a symbolic manipulation program (such as Mathematica). In this way the lowest few terms in the gradient expansion can be obtained in a straightforward manner.

Given the gradient expansion of the local DOS [which can be directly obtained from the second component of $\vec{\psi}(x)$ ], we can calculate the free energy by simple integrations. Using the fact that in the normal state $\rho(x ; \omega)=\rho_{0}$, the difference between the free energy densities in the gapped and normal state of our one-dimensional model at inverse temperature $\beta$ is given by $s \rho_{0} f(x)$, where

$$
\begin{aligned}
f(x) & =-\frac{1}{\beta} \operatorname{Re}\left[\int_{-\infty}^{\infty} d \omega\left[\psi_{2}(x ; \omega+i 0)-1\right] \ln \left(1+e^{-\beta \omega}\right)\right] \\
& =-\operatorname{Re}\left[\int_{-\infty}^{\infty} d \omega[i \Gamma(x ; \omega)-\omega] \frac{1}{e^{\beta \omega}+1}\right] \\
& =-\frac{2 \pi}{\beta} \operatorname{Re}\left[\sum_{\tilde{\omega}_{n}>0} \Gamma\left(x ; i \tilde{\omega}_{n}\right)-\tilde{\omega}_{n}\right] .
\end{aligned}
$$

Here, $\tilde{\omega}_{n}=(2 n+1) \pi / \beta$ are fermionic Matsubara frequencies, and

$$
i \Gamma(x ; \omega)=\int_{0}^{\omega} d \omega^{\prime} \psi_{2}\left(x ; \omega^{\prime}+i 0\right) .
$$

Note that $\mathcal{N}(x ; \omega)=-\rho_{0} \operatorname{Im} \Gamma(x ; \omega)$ is the integrated local DOS. In Eq. A.15) we have used the fact that $\psi_{2}(x ; \omega)$ is analytic in the upper half of the complex $\omega$-plane. The free energy functional of the FGM is now given by

$$
F\left\{\Delta, \Delta^{*}\right\}=s \rho_{0} \int_{0}^{L} d x\left[f(x)+\frac{|\Delta(x)|^{2}}{2 \lambda}\right] .
$$

The second term is the field energy of the order parameter field and was already derived in Chapter 1 .

The above equations allow for a simple recursive calculation of the gradient expansion of the free energy. Systematically adding total derivatives to the expressions for the free energy (which do not change the bulk properties), we find the following expressions:

Zeroth order:

$$
\begin{gathered}
\rho^{(0)}(x ; \omega)=\rho_{0} \theta\left(\omega^{2}-|\Delta|^{2}\right) \frac{|\omega|}{\sqrt{\omega^{2}-|\Delta|^{2}}}, \\
F^{(0)}\left\{\Delta, \Delta^{*}\right\}=s \rho_{0} \int_{0}^{L} d x\left(-\frac{2 \pi}{\beta} \sum_{0<\tilde{\omega}_{n} \lesssim \varepsilon_{0}}\left[\sqrt{\tilde{\omega}_{n}^{2}+|\Delta|^{2}}-\tilde{\omega}_{n}\right]+\frac{|\Delta|^{2}}{2 \lambda}\right) .
\end{gathered}
$$

Second order:

$\rho^{(2)}(x ; \omega)=\rho_{0}|\omega| \theta\left(\omega^{2}-|\Delta|^{2}\right)\left[-\frac{5}{32} \frac{\left[\partial_{x}|\Delta|^{2}\right]^{2}}{\left(\omega^{2}-|\Delta|^{2}\right)^{\frac{7}{2}}}-\frac{1}{8} \frac{\left[\partial_{x}^{2}|\Delta|^{2}\right]-3\left|\partial_{x} \Delta\right|^{2}}{\left(\omega^{2}-|\Delta|^{2}\right)^{\frac{5}{2}}}\right]$, 


$$
F^{(2)}\left\{\Delta, \Delta^{*}\right\}=s \rho_{0} \int_{0}^{L} d x \frac{2 \pi}{\beta} \sum_{\tilde{\omega}_{n}>0}\left[\frac{1}{8} \frac{\left|\partial_{x} \Delta\right|^{2}}{\left(\tilde{\omega}_{n}^{2}+|\Delta|^{2}\right)^{\frac{3}{2}}}-\frac{1}{32} \frac{\left[\partial_{x}|\Delta|^{2}\right]^{2}}{\left(\tilde{\omega}_{n}^{2}+|\Delta|^{2}\right)^{\frac{5}{2}}}\right] .
$$

Fourth order:

$$
\begin{aligned}
& \rho^{(4)}(x ; \omega)=\rho_{0}|\omega| \theta\left(\omega^{2}-|\Delta|^{2}\right)\left[\frac{1155}{2048} \frac{\left[\partial_{x}|\Delta|^{2}\right]^{4}}{\left(\omega^{2}-|\Delta|^{2}\right)^{\frac{13}{2}}}\right. \\
&+\frac{42}{512} \frac{\left[\partial_{x}|\Delta|^{2}\right]^{2}\left(11\left[\partial_{x}^{2}|\Delta|^{2}\right]-15\left|\partial_{x} \Delta\right|^{2}\right)}{\left(\omega^{2}-|\Delta|^{2}\right)^{\frac{11}{2}}} \\
&+\frac{7}{128} \frac{5\left|\partial_{x} \Delta\right|^{4}+4\left[\partial_{x}|\Delta|^{2}\right]\left[\partial_{x}^{3}|\Delta|^{2}\right]-10\left[\partial_{x}\left(\left|\partial_{x} \Delta\right|^{2}\left[\partial_{x}|\Delta|^{2}\right]\right)\right]+3\left[\partial_{x}^{2}|\Delta|^{2}\right]^{2}}{\left(\omega^{2}-|\Delta|^{2}\right)^{\frac{9}{2}}} \\
&+\frac{1}{32} \frac{\left[\partial_{x}^{4}|\Delta|^{2}\right]-5\left[\partial_{x}^{2}\left|\partial_{x} \Delta\right|^{2}\right]+5\left|\partial_{x}^{2} \Delta\right|^{2}}{\left(\omega^{2}-|\Delta|^{2}\right)^{\frac{7}{2}}}, \quad(\mathrm{~A} .22) \\
& F^{(4)}\left\{\Delta, \Delta^{*}\right\}=s \rho_{0} \int_{0}^{L} d x \frac{2 \pi}{\beta} \sum_{\tilde{\omega}_{n}>0}\left[-\frac{35}{2048} \frac{\left[\partial_{x}|\Delta|^{2}\right]^{4}}{\left(\tilde{\omega}_{n}^{2}+|\Delta|^{2}\right)^{\frac{11}{2}}}+\frac{70}{512} \frac{\left|\partial_{x} \Delta\right|^{2}\left[\partial_{x}|\Delta|^{2}\right]^{2}}{\left(\tilde{\omega}_{n}^{2}+|\Delta|^{2}\right)^{\frac{9}{2}}}\right. \\
&\left.+\frac{1}{128} \frac{5\left|\partial_{x} \Delta\right|^{4}-10\left|\partial_{x} \Delta\right|^{2}\left[\partial_{x}^{2}|\Delta|^{2}\right]+\left[\partial_{x}^{2}|\Delta|^{2}\right]^{2}}{\left(\tilde{\omega}_{n}^{2}+|\Delta|^{2}\right)^{\frac{7}{2}}} \frac{1}{32} \frac{\left|\partial_{x}^{2} \Delta\right|^{2}}{\left(\tilde{\omega}_{n}^{2}+|\Delta|^{2}\right)^{\frac{5}{2}}}\right] \quad(\mathrm{A} .23)
\end{aligned}
$$

Note that the high-frequency cutoff $\varepsilon_{0}$ in Eq. (A.19) is necessary to regularize the free energy which is well-known from the theory of superconductivity [20]. 



\section{Appendix B}

\section{Generation of Colored Noise}

In this appendix we describe a simple Markovian algorithm to generate a typical sample path of colored noise described by an Ornstein-Uhlenbeck process. The algorithm works equally well to simulate a real or complex disorder potential with exponentially decaying covariance and higher correlation functions given by Wick's theorem. As an input we only need independent Gaussian random numbers which can easily be generated by the well-known Box-Muller algorithm. Finally, we discuss an alternative method which can also be used to generate non-Gaussian colored noise.

Fluctuations of the relevant degrees of freedom in non-equilibrium statistical physics are usually taken into account by adding a stochastic force to the deterministic equations of motion. The prototype stochastic differential equation has the form

$$
\frac{d v}{d t}=-a(v(t))+b(v(t)) X(t)
$$

This equation is known as the Langevin equation [34, 46] and can easily be generalized to a matrix equation. It was first introduced by Langevin to describe Brownian motion [22]. $v(t)$ denotes the relevant variable which usually is a function of time 1 Note, however, that in condensed matter systems the disorder is often considered to be stationary, and in one-dimensional systems a space-coordinate can play the role of time.

The fluctuating random force is often called noise and can be of different orign. Internal forces such as thermal fluctuations are usually assumed to be Gaussian with very small correlation times $\tau_{c}$. Since a finite expectation value $\langle X(t)\rangle={ }^{-} x$ can be incorporated into $a(v(t))$, it is no restriction to assume

$$
\langle X(t)\rangle=0
$$

\footnotetext{
${ }^{1}$ In the case of Brownian motion, the relevant variable $v(t)$ is the velocity of a heavy particle of unit mass, $a(v(t)) \equiv \alpha \cdot v(t)$ is the dissipative force due to friction, and $b(v(t)) X(t) \equiv X(t)$ is an additive random force.
} 
where $\langle\ldots\rangle$ signifies averaging over the probability distribution of $X(t)$. A Gaussian stochastic process with standard deviation $\sigma$ and correlation time $\tau_{c}$ is characterized by the covariance

$$
\left\langle X(t) X\left(t^{\prime}\right)\right\rangle=\sigma^{2} e^{-\left|t-t^{\prime}\right| / \tau_{c}},
$$

and all higher moments given by Wick's theorem. This process is called the OrnsteinUhlenbeck process [92], which by Doob's theorem (see for example van Kampen [46]) is essentially the only stationary Gaussian Markov process. The white-noise limit may be taken by letting $\tau_{c}$ go to 0 while keeping the quantity $D \equiv \sigma^{2} \tau_{c}$ constant. In this limit the covariance becomes diagonal, such that disorder at different times is uncorrelated and

$$
\left\langle X(t) X\left(t^{\prime}\right)\right\rangle=2 D \delta\left(t-t^{\prime}\right) .
$$

While the white noise limit usually leads to a good approximation of internal fluctuations, in the case of external fluctuations the relevant variables can vary substantially over the correlation time $\tau_{c}$. In this case it is essential to consider colored noise. Unfortunately, in most cases the finite correlation time leads to a serious complication when trying to solve the Langevin equation. Techniques which turn out to be successful in solving the white noise limit can only be applied after coupling the stochastic equations of motion to an extra equation which takes care of the finite correlation time. Often, the only way out lies in a numerical simulation of the stochastic process. It is therefore important to find a method to generate typical disorder realizations. In the following, we describe a very simple algorithm to generate a concrete sample path of the Ornstein-Uhlenbeck process with finite correlation time $\tau_{c}$ which can be useful in various applications. This algorithm can already be found in similar form in the mathematical literature on stochastic processes [28] and was also used in different physical situations such as the Kramers problem (see for example [30, 59]). For the generation of spatio-temporal colored noise see [33].

\section{Simple algorithm to generate Gaussian colored noise}

Independent Gaussian random numbers $Z_{n}$ with zero mean and unit variance can be generated by the Box-Muller algorithm [74].2 The following recursive algorithm (which we will refer to as Algorithm I) maps these onto real correlated Gaussian random numbers $X_{n} \equiv X\left(t_{n}\right)$ at the sample points $t_{n}\left(t_{0}<t_{1}<\ldots<t_{N-1}\right)$ with $\left\langle X_{n}\right\rangle=0$ and $\left\langle X_{m} X_{n}\right\rangle=\sigma^{2} e^{-\left|t_{m}-t_{n}\right| / \tau_{c}}$ :

$$
\begin{aligned}
& X_{0}=\sigma Z_{0}, \\
& X_{n}=\rho_{n} X_{n-1}+\sqrt{1-\rho_{n}^{2}} \sigma Z_{n},
\end{aligned}
$$

where the correlation coefficients $\rho_{n}$ are given by $\rho_{n}=e^{-\left|t_{n}-t_{n-1}\right| / \tau_{c}}$. Setting $\rho_{0}=0$, Eq. (B.5) is also included in Eq. (B.6). A sample path generated by this algorithm is

\footnotetext{
${ }^{2}$ Note that the Box-Muller algorithm needs a good number generator to generate independent uniformly distributed random numbers.
} 


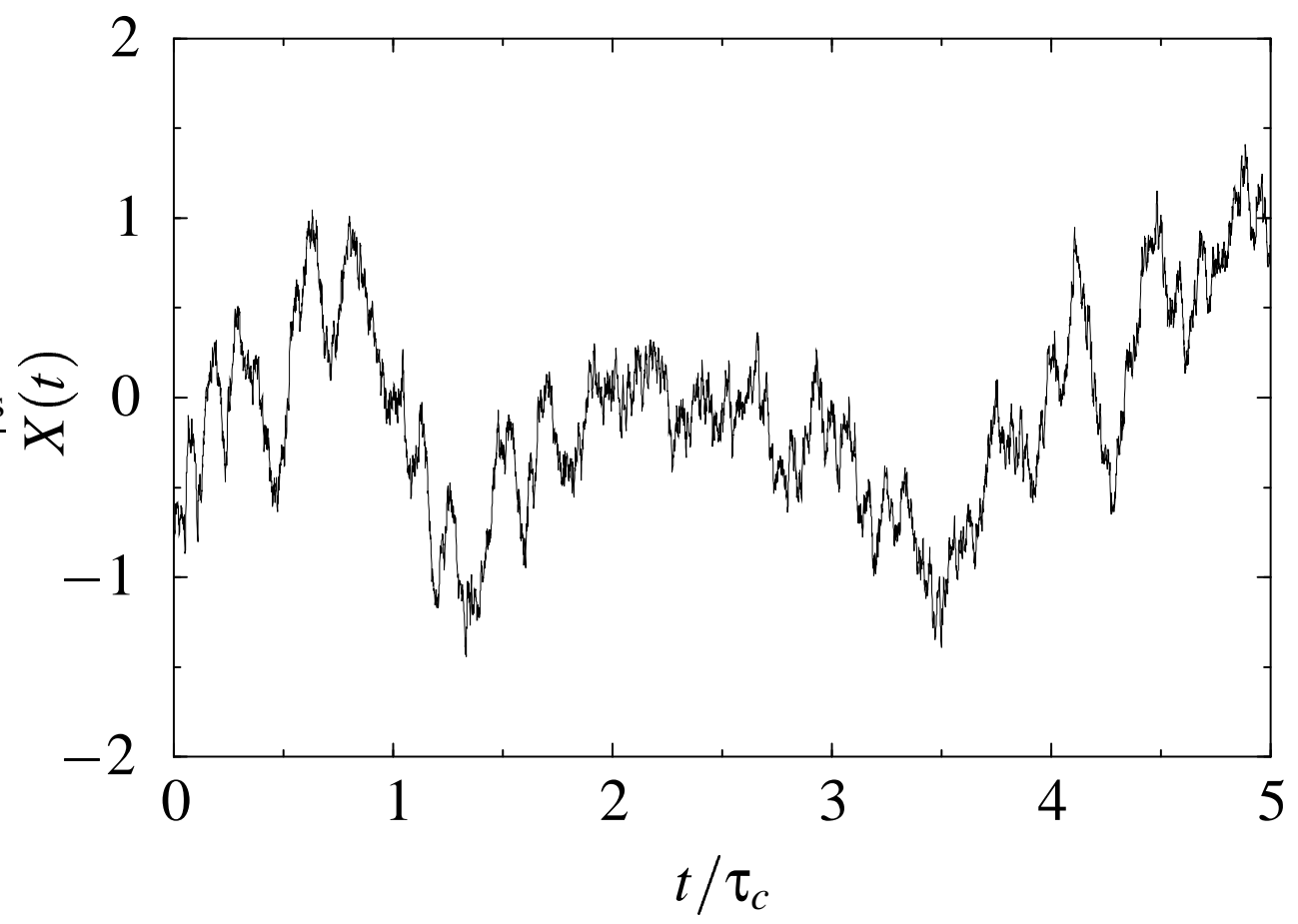

Figure B.1: Sample path of $X(t)$ with $\sigma=1$ as a function of $t / \tau_{c}$.

presented in Fig. B.1.

Using $\left\langle Z_{n}\right\rangle=0$ and $\left\langle Z_{m} Z_{n}\right\rangle=\delta_{m n}$, it is easy to see recursively from Eqs. (B.5) and (B.6) that the first two moments of $X_{n}$ are in fact given by $\left\langle X_{n}\right\rangle=0$ and $\left\langle X_{m} X_{n}\right\rangle=$ $\sigma^{2} e^{-\left|t_{m}-t_{n}\right| / \tau_{c}}$. Because the $X_{n}$ 's are given by a linear combination of the Gaussian random variables $X_{n}$ and a linear combination can only turn one Gaussian distribution into another Gaussian distribution [28], the $X_{n}$ 's also have to be Gaussian random variables. Higher correlation functions are therefore given by Wick's theorem [46].

It is also easy to generalize the above Algorithm I to a complex disorder potential. In this case one would like to have $\langle X(t)\rangle=0,\left\langle X(t) X^{*}\left(t^{\prime}\right)\right\rangle=\sigma^{2} e^{-\left|t-t^{\prime}\right| / \tau_{c}}$ and $\left\langle X(t) X\left(t^{\prime}\right)\right\rangle=0$. Generating $\operatorname{Re} X_{n}$ and $\operatorname{Im} X_{n}$ independently as before, one sees that to get the desired correlation functions one has to replace $\sigma$ by $\sigma / \sqrt{2}$. Since $X_{n}=\operatorname{Re} X_{n}+i \operatorname{Im} X_{n}$ only depends linearly on $\operatorname{Re} X_{n}$ and $\operatorname{Im} X_{n}$, the complex $X_{n}$ are also Gaussian random variables.

\section{Non-Gaussian colored noise}

The above Algorithm I is very simple and proves to be succesful in generating Gaussian colored noise. However, external fluctuations do not have to be Gaussian, and there might be a need to generate a typical chain characterized by different statistics. 
Let us now describe an algorithm based on an expansion of a stochastic process in terms of harmonic functions [23] which in the following we will refer to as Algorithm II. If $S(\omega)$ represents the power spectrum of the stochastic process, a typical sample path may be generated for large $N$ by (see [18, 15])

$$
X(t)=\sqrt{2} \sum_{n=0}^{N-1}\left[2 S\left(\omega_{n}\right) \Delta \omega\right]^{1 / 2} \cos \left(\omega_{n} t+\phi_{n}\right) .
$$

Here, the $\phi_{n}$ are independent random phases which are uniformly distributed over the interval $(0,2 \pi), \Delta \omega=\omega_{\max } / N$, where $\omega_{\max }$ is an upper cutoff of the noise spectrum, and $\omega_{n}=n \Delta \omega$. Algorithm II has the advantage that it is applicable to an arbitrary given spectrum $S(\omega)$. The spectrum of Gaussian colored noise can be found by taking the Fourier transform of Eq. (B.3), resulting in

$$
S(\omega)=\frac{1}{\pi} \frac{\sigma^{2} \tau_{c}}{1+\omega^{2} \tau_{c}^{2}}
$$

In comparison to Algorithm I, which unfortunetely only works to generate a sample path of an Ornstein-Uhlenbeck process, Algorithm II has the disadvantage that for it to become accurate, both $\omega_{\max }$ and then $N$ have to be chosen sufficiently large. Even when using a fast Fourier transform which results in $O\left(N \log _{2} N\right)$ operations [74], this can lead to large computation times. In addition, the sample paths are always periodic with period $2 \pi N / \omega_{\max }$, which can lead to further complications. For a more quantitative comparison between the two Algorithms see the Comment by Manella and Palleschi [60] on Ref. [15].

In summary, we have described a very simple algorithm to simulate a real or complex Ornstein-Uhlenbeck process and an alternative algorithm which is not restricted to generate Gaussian colored noise. When generating Gaussian colored noise, the advantage of the former in comparison to the latter is that it takes advantage of the Markov property of the Ornstein-Uhlenbeck process: To generate $X_{n}$ we only need to know $X_{n-1}$. When numerically solving an initial value problem of a stochastic differential equation, the disorder may be simultaneously generated with the propagation of the desired solution. In addition, arbitrary long chains can be easily generated. We have used the described algorithm to generate the fluctuating order parameter field $\Delta(x) \equiv X(t)$ of the so-called fluctuating gap model (see Chapter 4 and Ref. [11]). The above method enabled us to calculate the density of states for arbitrary correlation lengths $\xi \equiv \tau_{c}$ with unprecedented numerical accuracy. The algorithm, however, should be useful in all contexts where there is a need to generate colored noise described by an OrnsteinUhlenbeck process. 


\section{Bibliography}

[1] A. A. Abrikosov, L. P. Gorkov, and I. E. Dzyaloshinskii, Methods of Quantum Field Theory in Statistical Physics, (Dover, New York, 1963).

[2] A. A. Abrikosov and I. A. Ryzhkin, Zh. Eksp. Teor. Fiz. 71, 1204 (1976) [Sov. Phys. JETP 44, 630 (1976)].

[3] A. A. Abrikosov and E. A. Dorotheyev, J. Low Temp. Phys. 46, 53 (1982).

[4] M. Abramowitz and I. A. Stegun, Handbook of mathematical functions, (Dover Publications, New York, 1965).

[5] P. W. Anderson, Phys. Rev. 109, 1492 (1958).

[6] N. W. Ashcroft und N. D. Mermin, Solid State Physics, (Saunders College Publishing, Philadelphia 1988).

[7] L. Bartosch and P. Kopietz, Phys. Rev. B 55, 15360 (1997).

[8] L. Bartosch and P. Kopietz, Phys. Rev. Lett. 82, 988 (1999).

[9] L. Bartosch and P. Kopietz, Phys. Rev. B 59, 5377 (1999).

[10] L. Bartosch and P. Kopietz, Phys. Rev. B 60, 7452 (1999).

[11] L. Bartosch and P. Kopietz, Phys. Rev. B 60, 15488 (1999).

[12] L. Bartosch and P. Kopietz, Phys. Rev. Lett. 84, 2547 (2000).

[13] L. Bartosch and P. Kopietz, cond-mat/0006346 (to appear in European Journal of Physics B).

[14] L. Bartosch and P. Kopietz, cond-mat/0006347 (submitted to Physical Review Letters).

[15] K. Y. R. Billah and M. Shinozuka, Phys. Rev. A 42, 7492 (1990).

[16] S. A. Brazovskii and I. E. Dzyaloshinskii, Zh- Eksp. Teor. Fiz. 71, 2338 (1976) [Sov. Phys. JETP 44, 1233 (1976)]. 
[17] J. E. Bunder and R. H. McKenzie, Phys. Rev. B 60, 344 (1999).

[18] J. Cacko, Random processes: measurement, analysis and simulation, (Elsevier, Amsterdam, 1988).

[19] F. J. Dyson, Phys. Rev. 92, 1331 (1953).

[20] G. Eilenberger, Z. Phys. 182, 427 (1965).

[21] G. Eilenberger, Z. Phys. 214, 195 (1968).

[22] A. Einstein, Ann. Phys. (Leipzig) 17, 549 (1905).

[23] A. Einstein and L. Hopf, Ann. Phys. (Leipzig) 33, 1095 (1910).

[24] K. B. Efetov, Supersymmetry in disorder and chaos, (Cambridge University Press, Cambridge, 1997).

[25] V. J. Emery and S. A. Kivelson, Nature 374, 434 (1995).

[26] V. J. Emery and S. A. Kivelson, Phys. Rev. Lett. 74, 3253 (1995).

[27] M. Fabrizio and R. Mélin, Phys. Rev. Lett. 78, 3382 (1997).

[28] W. Feller, An Introduction to Probability Theory and its Applications, Vol. II, 2nd ed. (Wiley, New York, 1971).

[29] H. J. Fischbeck and R. Hayn, Phys. Status Solidi B 158, 565 (1990).

[30] R. F. Fox, I. R. Gatland, R. Roy and G. Vemuri, Phys. Rev. A 38, 5938 (1988).

[31] J. Fröhlich, Proc. Royal Soc. London Ser. A 233, 296 (1954).

[32] A. A. Golub and Y. M. Chumakov, Fiz. Nizk. Temp. 5, 900 (1979) [Sov. J. Low Temp. Phys. 5, 427 (1980)].

[33] J. García-Ojalvo, J. M. Sancho and L. Ramírez-Piscina, Phys. Rev. A, 46, 4670 (1992).

[34] C. W. Gardiner, Handbook of Stochastic Methods for physics, chemistry, and the natural sciences, (Springer-Verlag, Berlin, Heidelberg, 1983).

[35] I. S. Gradshteyn and I. M. Ryzhik, Table of Integrals, Series, and Products (Academic Press, San Diego, 1980).

[36] G. Grüner, Rev. of Mod. Phys. 60, 1129 (1988).

[37] G. Grüner, Density Waves in Solids, (Addison-Wesley, Reading, 1994).

[38] G. Grüner, Rev. of Mod. Phys. 66, 1 (1994). 
[39] B. I. Halperin, Phys. Rev. 139, A104 (1965).

[40] R. Hayn and W. John, Z. Phys. B 67, 169 (1987).

[41] R. Hayn and H. J. Fischbeck, Z. Phys. B 76, 33 (1989).

[42] R. Hayn and J. Mertsching, Phys. Rev. B 54, R5199 (1996).

[43] C. Itzykson and J. C. Drouffe, Statistical Field Theory, Vol. II, (Cambridge University Press, Cambridge, 1989).

[44] D. C. Johnston, Phys. Rev. Lett. 52, 2049 (1984).

[45] D. C. Johnston, M. Maki and G. Grüner, Solid State Commun. 53, 5 (1985).

[46] N. G. van Kampen, Stochastic Processes in Physics and Chemistry, (NorthHolland, Amsterdam, 1981).

[47] W. Kohn, Phys. Rev. Lett. 2, 393 (1959).

[48] P. Kopietz and G. E. Castilla, Phys. Rev. Lett. 76, 4777 (1996).

[49] P. Kopietz and G. E. Castilla, Phys. Rev. Lett. 78, 314 (1997).

[50] P. Kopietz, Bosonization of Interacting Fermions in Arbitrary Dimensions, (Springer, Berlin, 1997).

[51] P. Kopietz, Phys. Rev. Lett. 81, 2120 (1998).

[52] S. Kos and M. Stone, Phys. Rev. B 59, 9545 (1999).

[53] I. Kosztin, S. Kos, M. Stone, and A. J. Leggett, Phys. Rev. B 58, 9365 (1998).

[54] E. Z. Kuchinskii and M. V. Sadovskii, Zh. Eksp. Teor. Fiz. 115, 1765 (1999) [Sov. Phys. JETP 88, 968 (1999)].

[55] C. G. Kuper, Proc. Royal Soc. London A 227, 214 (1955).

[56] A. I. Larkin and Y. N. Ovchinnikov, Zh. Eksp. Teor. Fiz. 55, 2262 (1968) [Sov. Phys. JETP 28, 1200 (1969)].

[57] P. A. Lee, T. M. Rice and P. W. Anderson, Phys. Rev. Lett. 31, 462 (1973).

[58] I. M. Lifshits, S. A. Gredeskul, and L. A. Pastur, Introduction to the Theory of Disordered Systems, (Wiley, New York, 1988).

[59] R. Manella and V. Palleschi, Phys. Rev. A 40, 3381 (1989).

[60] R. Manella and V. Palleschi, Phys. Rev. A 46, 8028 (1992). 
[61] R. H. McKenzie, Phys. Rev. B 52, 16428 (1995).

[62] R. H. McKenzie, Phys. Rev. Lett. 77, 4804 (1996).

[63] R. H. McKenzie and D. Scarratt, Phys. Rev. B 54, R12709 (1996).

[64] N. D. Mermin and H. Wagner, Phys. Rev. Lett. 17, 1133 (1966).

[65] J. Mertsching, Phys. Status Solidi B 174, 129 (1992).

[66] A. Millis and H. Monien, Phys. Rev. B 61, 12496 (2000).

[67] A. Millis and H. Monien, Phys. Rev. Lett. 84, 2546 (2000).

[68] M. Mostovoy and J. Knoester, Int. J. Mod. Phys. B 13, 1601 (1999).

[69] N. F. Mott and W. D. Twose, Adv. Phys. 10, 107 (1961).

[70] J. W. Negele and H. Orland, Quantum Many-Particle Physics, (Addison-Wesley, Redwood City, 1988).

[71] A. A. Ovchinnikov and N. S. Erikhman, Zh. Eksp. Teor. Fiz. 73, 650 (1977) [Sov. Phys. JETP 46, 340 (1977)].

[72] R. E. Peierls, Quantum Theory of Solids (Oxford University Press, New York, 1955)

[73] M. E. Peskin and D. V. Schroeder, An Introduction to Quantum Field Theory, (Addison-Wesley, Reading, 1995), chapter 15.

[74] W. H. Press, B. P. Flannery, S. A. Teukolsky and W. T. Vetterling, Numerical Recipes in C, (2nd ed., Cambridge University Press, Cambridge, 1992).

[75] M. J. Rice and S. Strässler, Solid State Commun. 13, 125 (1973).

[76] M. J. Rice and S. Strässler, Solid State Commun. 13, 1389 (1973).

[77] M. V. Sadovskii, Zh. Eksp. Teor. Fiz. 66, 1720 (1974) [Sov. Phys. JETP 39, 845 (1974)].

[78] M. V. Sadovskii, Zh. Eksp. Teor. Fiz. 77, 2070 (1979) [Sov. Phys. JETP 50, 989 (1979)].

[79] D. J. Scalapino, M. Sears and R. A. Ferrell, Phys. Rev. B 9, 3409 (1972).

[80] J. Schmalian, D. Pines and B. Stojković, Phys. Rev. Lett. 80, 3839 (1998).

[81] J. Schmalian, D. Pines and B. Stojković, Phys. Rev. B 60, 667 (1999).

[82] N. Schopohl, cond-mat/9804064, (unpublished). 
[83] J. R. Schrieffer, Theory of Superconductivity, (Benjamin, New York, 1964).

[84] J. Schwinger, Phys. Rev. 128, 2425 (1962).

[85] M. Steiner, M. Fabrizio and A. O. Gogolin, Phys. Rev. B 57, 8290 (1998);

[86] M. Steiner, Yang-Chen, M. Fabrizio and A. O. Gogolin, Phys. Rev. B 59, 14848 (1999).

[87] O. Tchernyshyov, Phys. Rev. B 59, 1358 (1999).

[88] L. Tewordt, Phys. Rev. 132, 595 (1963).

[89] L. Tewordt, Z. Phys. 180, 385 (1964).

[90] M. Tinkham, Introduction to Superconductivity, (Mc Graw-Hill, New York, second edition 1996).

[91] D. J. Thouless, J. Phys. C 5, 77 (1972).

[92] G. E. Uhlenbeck and L. S. Ornstein, Phys. Rev. 36, 823 (1930); reprinted in N. Wax, selected papers on noise and stochastic processes, (Dover Publications, New York, 1954).

[93] D. Waxman, Ann. Phys. (N.Y.) 223, 129 (1993).

[94] N. R. Werthamer, Phys. Rev. 132, 663 (1963).

[95] N. R. Werthamer, The Ginzburg-Landau Equations and Their Extensions in Superconductivity, edited by R. D. Parks (Marcel Dekker, New York, 1969).

[96] W. Wonneberger and R. Lautenschlager, J. Phys. C 9, 2865 (1976). 



\section{Lebenslauf}

$\begin{array}{ll}\text { Name: } & \text { Bartosch } \\ \text { Vorname: } & \text { Lorenz } \\ \text { Geburtstag: } & \text { 1. November } 1969 \\ \text { Geburtsort: } & \text { Nordhorn }\end{array}$

Schule: $\quad$ 1976-1980: Grundschule Melbeck 1980-1982: Orientierungsstufe Embsen 1982-1989: Gymnasium Wilhelm-Raabe-Schule, Lüneburg

Abitur: $\quad$ 17. Mai 1989 an der Wilhelm-Raabe-Schule, Lüneburg

Wehrdienst: $\quad$ 1. Juni 1989 bis 31. August 1990 als Hubschraubermechaniker

Studium: $\quad$ Physik (Diplom), sowie Mathematik und Physik (Lehramt) WS 1990/91-SS 1992: Georg-August-Universität, Göttingen WS 1992/93-SS 1993: Akademisches Auslandsjahr an der University of California, Santa Cruz WS 1993/94-SS 1997: Georg-August-Universität, Göttingen WS 1997/98: Besuch der Arbeitsgruppe von Prof. Sudip Chakravarty an der University of California, Los Angeles SS 1998-SS 2000: Georg-August-Universität, Göttingen

Examen: $\quad$ Vordiplom im Studiengang Physik am 23. Juni 1992 Zwischenprüfung Mathematik am 7. Juli 1994

Diplomhauptprüfung Physik am 1. November 1996 Thema der Diplomarbeit bei Prof. Dr. Kurt Schönhammer: Korrelierte Fermionen in gekoppelten Ketten

Promotion: $\quad$ Seit dem WS 1996/97 Wissenschaftlicher Mitarbeiter am Institut für Theoretische Physik der Georg-August-Universität, Göttingen, und Promotion bei Priv. Doz. Dr. Peter Kopietz. Thema der Doktorarbeit: Singularities and Pseudogaps in the Density of States of the Fluctuating Gap Model 